\title{
An investigation of behavioural and electrophysiological markers of integration in learning of novel names for novel concepts
}

\section{Maria Korochkina ${ }^{* 1,2}$, Lyndsey Nickels ${ }^{1}$, Audrey Bürki ${ }^{3}$}

\author{
${ }^{1}$ School of Psychological Sciences, Macquarie University, \\ 16 University Avenue, NSW 2109 Australia \\ ${ }^{2}$ International Doctorate for Experimental Approaches to Language and Brain (IDEALAB): \\ Universities of Potsdam (Germany), Groningen (Netherlands), \\ Newcastle (UK) and Macquarie University (Australia) \\ ${ }^{3}$ Cognitive Sciences, Department of Linguistics, University of Potsdam, \\ Karl-Liebknecht-Straße 24-25, 14476 Potsdam, Germany
}

\begin{abstract}
The Complementary Learning Systems model of word learning proposes that newly learned words that have been integrated into semantic memory can interact with familiar words during lexical selection. The study reported here is the first to examine how behavioural markers of integration map onto electrophysiological markers of integration in a version of the semantic priming paradigm that is assumed to rely primarily on automatic semantic processing. 71 young healthy adults learned novel names for two sets of novel concepts, one set on each of two consecutive days. Learning was followed by a continuous primed lexical decision task with EEG measures. The behavioural data was analysed with Bayesian Linear Mixed Effects models, while, for the electrophysiological data, two types of analyses were conducted: Bayesian Distributional Regression models were used to analyse mean amplitude in two pre-defined spatiotemporal windows (N400 and LPC), whereas the Mass Univariate analysis was run to explore other time points and regions. We found evidence against priming effects in either spatiotemporal window or in the behavioural data. Nonetheless, there was evidence for differential processing of the novel names depending on the length of the consolidation period (0-day vs 1 -day). We take these findings to indicate that neither the 0 -day nor the 1-day consolidation period was sufficient for integration in our study and that, 24 hours after exposure to novel words, the system still relies on episodic memory to distinguish between these novel words (learned 24h ago), those learned more recently and those that haven't been seen before.
\end{abstract}

Keywords: word learning, EEG, episodic memory, semantic memory, integration

\footnotetext{
${ }^{*}$ Corresponding author (maria.korochkina@hdr.mq.edu.au) .
} 


\section{Introduction}

1 Learning of words is a frequent experience in our lives. While the rate of words learned may

2 be at its highest during the school years (e.g., Anglin, 2000), vocabulary development continues

3 throughout adulthood (Brysbaert et al., 2016; Keuleers et al., 2015), with most adults also

4 learning new words in languages other than their first language. Yet, despite the early onset

5 and the ubiquity of word learning, its multifaceted nature makes it no trivial task. In addition

6 to learning a new phonological form, the concept it refers to and the association between them,

7 word acquisition entails integrating new words in a network of thousands of already familiar

8 words and connections (e.g., McMurray et al., 2016). In this study, we focused on the fact that

9 there are still many questions to be answered regarding how this integration occurs. Specifically, we sought to broaden the current understanding of how behavioural markers of integration map onto electrophysiological markers of integration in learning of novel names for novel concepts.

An account of the processes by which new words are integrated into the semantic network and achieve the same status as already familiar words was proposed by Davis and Gaskell (2009). Davis and Gaskell's account is based on the Complementary Learning Systems (CLS) model, a connectionist model of learning advanced by James McClelland and collaborators (Kumaran et al., 2016; McClelland et al., 1995; McClelland, 2013; McClelland et al., 2020; O'Reilly et al., 2014). This account suggests that word learning occurs over two stages. The first stage relies on the episodic memory system that is thought to reside in the medial temporal lobe, specifically, the hippocampus. In contrast, the second stage is dependent on the semantic memory system subserved by the neocortex (e.g., Squire, 2004, but see Duff et al., 2020, for a recent review on the hippocampal contribution to maintenance and retrieval of semantic memories). Whereas episodic memory refers to the capacity to learn, store and evoke detailed information about specific events together with their spatiotemporal context (Dickerson \& Eichenbaum, 2010), semantic memory is viewed as a store of our knowledge about the world and the meanings of verbal symbols (i.e., words) that we employ to make use of this knowledge (see Kumar, 2021, for a recent review).

The CLS model is rooted in the notion that new memories usually start as episodic and later become abstracted away from the spatiotemporal context they were acquired in and integrated into the existing knowledge networks (e.g., Meeter \& Murre, 2004). In word learning, Lindsay 
and Gaskell (2010) suggest that the first episodic representations are initially in the form of sparse neural representations about the word's properties such as its spelling, pronunciation, meaning as well as about the context it is perceived in (this stage of memory development is commonly referred to as encoding, see, e.g., Hasselmo et al., 2007; Davachi \& Dobbins, 2008). The CLS model of word learning also postulates that the lexical status of the new words differs from that of already familiar words until the newly formed episodic memory traces have been consolidated, i.e., transformed into long-term memory (Davis \& Gaskell, 2009). Because consolidation is believed to slow down the rate of forgetting, reinforce resistance to interference from other stimuli (e.g., Dudai \& Eisenberg, 2004; Wixted, 2004), facilitate access to the meanings of the new words and enhance the extent to which their lexical representations impact lexical processing of familiar words, it is considered a pre-condition for integration of new words into the lexicon (Davis \& Gaskell, 2009; Lindsay \& Gaskell, 2010, and see Leach \& Samuel, 2007, for a similar two-stage model of word learning). An extensive literature examining the optimal conditions for consolidation suggests that, while consolidation is actively promoted in sleep, wakefulness does not preclude it (e.g., Rasch \& Born, 2013; Stickgold \& Walker, 2005).

Numerous studies have tested aspects of the CLS framework using learning of (meaningless) novel word forms in both spoken (e.g., Bakker et al., 2014; Davis et al., 2009; Dumay \& Gaskell, 2007, 2012; Dumay et al., 2004; Gaskell \& Dumay, 2003; Kapnoula et al., 2015; Kapnoula \& McMurray, 2016; Lindsay \& Gaskell, 2013; Tamminen \& Gaskell, 2008; Tamminen et al., 2010) and written (e.g., Bakker et al., 2014; Bowers et al., 2005; Walker et al., 2010) modalities as well as acquisition of novel names for novel concepts (e.g., Bakker et al., 2015; Balass et al., 2010; Batterink \& Neville, 2011; Borovsky et al., 2010, 2012, 2013; Clay et al., 2007; Dumay et al., 2004; Fang \& Perfetti, 2017; Liu \& van Hell, 2020; Perfetti et al., 2005; Tamminen \& Gaskell, 2013; van der Ven et al., 2015) and novel names for familiar concepts (e.g., Breitenstein et al., 2007; Dobel et al., 2009; Elgort, 2011; Elgort \& Warren, 2014; Geukes et al., 2015; Korochkina et al., 2021; McGregor, 2014; McLaughlin et al., 2004; Mestres-Missé et al., 2007, 2008; Tham et al., 2015) in young adults (for work with older adults see, e.g., Kurdziel et al., 2017, and, for work with children, e.g., Henderson et al., 2012, 2013; James et al., 2017, 2019; Weighall et al., 2016). Employing behavioural, electrophysiological, neuroimaging and eye-tracking techniques to measure integration, this extensive literature has supported some of the assumptions of the original CLS account and challenged others (see, e.g., McMurray et al., 2016, for a review). 
In studies focusing on learning of novel names for novel concepts, integration has been almost exclusively examined with a semantic priming paradigm in word recognition (but see Clay et al., 2007, for work on word production, and Borovsky et al., 2010, for use of a sentence processing task). In a standard (paired) version of this paradigm, participants are presented with pairs of stimuli (primes and targets) and asked to make either lexical decision (word-nonword discrimination) or semantic-relatedness judgments (i.e., whether two words are semantically related) to the targets. Importantly, the relationship between the primes and the targets is manipulated such that the prime is either semantically related to the target (e.g., sofa and bed) or not (e.g., hat and bed). When targets are preceded by semantically related as opposed to unrelated primes, participants make target judgements faster, a phenomenon that is referred to as a semantic priming effect (see McNamara, 2005, for an extensive review). This is thought to be because the prime activates its semantic representation, and this activation then spreads to representations of semantically related words, including the (semantically related) target (e.g., Collins \& Loftus, 1975; Posner \& Snyder, 1975). This pre-activation of the target means that less additional activation is required when the semantically related target word is encountered, resulting in faster response times than when the target is semantically unrelated to the prime (but see de Wit \& Kinoshita, 2015, and Neely, 1991, for an alternative account). Crucially, when the prime is a newly learned novel word, it is believed to be able to spread semantic activation to familiar words only if it had been integrated into the lexical-semantic network (e.g., Davis \& Gaskell, 2009; Lindsay \& Gaskell, 2010; Tamminen \& Gaskell, 2013).

In the following sections, we provide an overview of the literature that has used the semantic priming paradigm to study integration of novel names for novel concepts. We start by reviewing behavioural studies and then move on to studies examining the electrophysiology of the integration process.

\section{Behavioural studies on integration of novel names for novel concepts}

Several studies have addressed integration of novel names for novel concepts, however, no clear picture has emerged. Dumay et al. (2004, Experiment 1) taught their participants novel words (e.g., cathedruke) that were derived from familiar English words (e.g., cathedral; base words) and given meaning from sentence contexts, for example, "A cathedruke is a variety of vegetable" and

"The cook served the boiled cathedruke with a steak and baked potatoes". In a continuous primed 
lexical decision task (word-nonword discrimination at both primes and targets) administered one week after training, following novel word primes, Dumay and colleagues observed shorter response times to the semantic superordinates (e.g., vegetable) of the novel words as compared to semantically unrelated category names (i.e., semantic priming) as well as longer response times to the base words (cathedral) than to familiar English words that had no phonological and orthographic overlap with the novel words (i.e., form priming). Because these effects were not present either immediately after or 24 hours after training, Dumay et al. (2004) concluded that integration of meaningful novel words may take up to one week.

Tamminen and Gaskell (2013) pointed out a methodological issue with Dumay et al.'s study: the novel words (primes) had co-occurred with the semantic categories (targets) in the sentence contexts presented as part of training. They argued this could mean that the semantic priming effect observed in the Dumay et al. study might have been at least partly driven by wordto-word (episodic) association rather than integration into semantic memory. Tamminen and Gaskell (2013) addressed this issue in two experiments. Participants learned two sets of words in two experimental sessions (remote and recent sets, respectively), conducted either on two consecutive days (1-day consolidation opportunity group) or with a between-session interval of one week (7-day consolidation opportunity group). Both groups performed a paired primed lexical decision task (for both sets) immediately after learning of the recent set. Surprisingly, the semantic priming effect did not differ either across the groups or across the sets, regardless of whether the primes were visible (Experiment 1) or masked (Experiment 2). However, when they combined the data from the two experiments (in attempt to increase statistical power), the interaction between priming and time of testing was significant, with a stronger priming effect after a 7-day than after a 0-day consolidation period (the 1-day vs. 0-day consolidation period was not tested). Tamminen and Gaskell (2013), thus, inferred that novel names for novel concepts can be embedded with other lexical-semantic representations relatively quickly, with the initially weak semantic priming effect growing stronger over time.

Following Tamminen \& Gaskell's work, the 1-day vs. 0-day consolidation periods were directly compared by van der Ven et al. (2015). They found a main effect of day, but only in the by-participants analysis: there was no difference $(0 \mathrm{~ms})$ in response times between semantically related and unrelated targets immediately after training, but, 24 hours after training, a relatively small (10 ms) priming effect emerged. 
In sum, whereas van der Ven et al. (2015) have argued that a 1-day consolidation period may be enough for integration, Dumay et al. (2004) suggested a more protracted time course of one week (but with potential methodological issues), while Tamminen and Gaskell (2013)'s findings were inconclusive with respect to the contrast between the 1-day and the 0 -day consolidation periods.

The nature of the behavioural priming effect also remains unclear and, in particular, whether this can be considered a reliable marker of integration. This is because, although it has been claimed that only integrated novel words can compete with familiar words for lexical selection (e.g., Davis \& Gaskell, 2009; Lindsay \& Gaskell, 2010; Qiao et al., 2009), there is evidence that episodic and semantic memory representations actively interact with each other even in nonepisodic processes such as priming, especially for recently acquired information (e.g., Kumaran et al., 2016; Moscovitch et al., 2016; Winocur \& Moscovitch, 2011). In attempt to shed light on this issue, several studies have examined the neural signatures of the semantic priming effects with newly trained words, and we now turn to these studies.

\section{Electrophysiological studies on integration of novel names for novel concepts}

Studies examining the neural signatures of integration have primarily focused on two ERP components, the N400 and the Late Positive Component (LPC). The N400 is a negative brain potential with a centro-parietal distribution and a slight right-hemisphere bias that peaks approximately $400 \mathrm{~ms}$ after the onset of a conceptually meaningful stimulus (e.g., a word, picture, face, sound; see Kutas \& Federmeier, 2011, for a review). Its amplitude typically becomes smaller when the stimulus is congruent with the context it appears in (e.g., the preceding word or part of a sentence). For instance, in the semantic priming paradigm, a larger (i.e., more negative) N400 response is typically observed when the target word (e.g., cat) is preceded by a semantically unrelated (e.g., oak) as opposed to a semantically related word (e.g., dog); this effect is usually referred to as the $N_{4} 00$ priming effect. While the functional basis of this effect is still debated, one of the most prominent theories posits that it indexes retrieval of a word from semantic memory (e.g., Brouwer et al., 2017; Deacon et al., 2004; Delogu et al., 2019; Kutas \& Federmeier, 2000, 2011; Lau et al., 2008, but see, e.g., Brown \& Hagoort, 1993; Hagoort, 2008; Rabovsky et al., 2018, for other accounts). When a word is encountered (e.g., dog), the system makes predictions about the upcoming stimulus and 
pre-activates words that are semantically related to the stimulus that is currently being processed (e.g., wolf, cat, horse; see, e.g., Szewczyk \& Schriefers, 2018). Therefore, when the upcoming word (e.g., oak) is not among the pre-activated words, a larger (more negative) N400 is elicited than when the upcoming word is one of the pre-activated words (e.g., cat).

The LPC is a positive-going wave with a bilateral parietal distribution, peaking at around $600 \mathrm{~ms}$ post-stimulus, and is believed to be a marker of episodic memory retrieval (see, e.g., Rugg, 1995, for a review). In the recognition memory literature, it has been observed that stimuli that are correctly recognised as having been encountered in a prior study list ('old' stimuli) evoke a larger (i.e., more positive) LPC response than correctly rejected new stimuli, or new stimuli incorrectly judged as old or old stimuli that are unrecognised (e.g., Curran, 1999; Rugg \& Curran, 2007; Senkfor \& Van Petten, 1998). This old/new recollection effect has also been found when the participants are not explicitly told to make memory judgements, in for example, repetition priming paradigms (e.g., Kazmerski \& Friedman, 1997; Paller et al., 1995; Van Petten \& Senkfor, 1996). In the literature on semantic processing, the LPC has been linked to explicit processes in semantic retrieval, contextual integration and revision (e.g., Swaab et al., 1998; Van Petten et al., 1991). Consequently, reports of more positive LPC responses to semantically and associatively related as opposed to unrelated targets (labelled the LPC priming effect) have been interpreted as an index of controlled and strategic semantic processing in word recognition (e.g., Hill et al., 2002; Hoshino \& Thierry, 2012; Kandhadai \& Federmeier, 2010). The LPC has also been linked to other late positive components (e.g., P3 and P600), and we refer the reader to excellent reviews by Brouwer et al. (2012) and Van Petten and Luka (2012) on the differences and similarities between these ERP responses.

Like the behavioural literature, the electrophysiological literature on integration has yielded inconsistent results. Most studies used the primed semantic-relatedness judgement or standard (paired) lexical decision paradigms. While some studies have reported an N400 priming effect immediately after learning (Balass et al., 2010; Perfetti et al., 2005; and see Batterink \& Neville, 2011; Mestres-Missé et al., 2007, 2008, for studies on learning of novel names for familiar concepts), others have failed to observe the N400 effect immediately after, 24 hours after (Bakker et al., 2015; Liu \& van Hell, 2020) or even one week after exposure (Liu \& van Hell, 2020). Notably, in all studies where the N400 priming effect was obtained in the semantic-relatedness judgement task (Balass et al., 2010; Batterink \& Neville, 2011; Perfetti et 
al., 2005), trained novel names were used as primes, while, in those studies where this effect was absent (Bakker et al., 2015; Liu \& van Hell, 2020), trained novel names were targets. Yet, this issue has received no attention in the literature.

With respect to the LPC, both the old/new recollection effect and the LPC priming effect have been reported. Where an enhanced LPC response to novel versus familiar words was observed, it was interpreted as an index of correct recognition of previously encountered stimuli (Balass et al., 2010; Perfetti et al., 2005). In studies where greater positivity in this spatiotemporal window was observed to related as opposed to unrelated targets, it was taken to index non-automatic processes during lexical retrieval and possibly incomplete integration (Bakker et al., 2015; Borovsky et al., 2013; Liu \& van Hell, 2020). In addition, some studies have found a stronger LPC response to familiar words as compared to trained novel words and claimed that it reflected poor recollection of the novel words' meanings (Bakker et al., 2015; Liu \& van Hell, 2020).

Importantly, the same caveats as those from the behavioural literature must be considered in the case of the electrophysiological studies. For instance, in Perfetti et al. (2005), many of the targets (familiar English words) had occurred as part of the definitions of the rare words the participants had been trained on, thus making it possible that the observed N400 effects were at least partly due to word-to-word association priming. While this confound was not present in Balass et al. (2010), both Perfetti et al. and Balass et al. used the semantic-relatedness judgement task, which, as we have argued above, is unlikely to measure (solely) automatic processes of lexical retrieval. A similar argument was also advanced by Batterink and Neville (2011) who asserted that the semantic-relatedness judgement task relies on the explicit memory system and requires conscious recollection. This was supported by the fact that, when they contrasted performance on the semantic-relatedness judgment task and the paired primed lexical decision task immediately after training, the N400 priming effect was observed only in the semanticrelatedness task. Batterink and Neville, therefore, suggested that the primed lexical decision task is a much more reliable measure of implicit memory and argued that explicit representations of novel word meanings can develop rapidly, whereas implicit representations may require more time to emerge. This argument contrasts with that of Borovsky et al. (2013), who postulated that implicit representations of novel words can be rapidly integrated after a very brief exposure, but only in the right hemisphere, whereas the engagement of the left hemisphere in meaning 
representation increases only with continued experience and better knowledge of the word (and see Ince \& Christman, 2002, for the hemispheric representation account). This conclusion was based on their finding of both the N400 and the LPC priming effects in a paired primed lexical decision task (administered immediately after exposure to novel words) for targets shown in the left visual field and only the LPC priming effect for targets shown in the right visual field (but note that in their earlier study, where the manipulation of the visual field was not present, only the N400, but not the LPC priming effect was observed; Borovsky et al., 2012).

Critically though, while all studies discussed above used different training procedures, the two studies by Borovsky and colleagues differ in one more aspect: the priming task was interleaved with training. Specifically, instead of first completing the training task (reading of strongly or weakly constraining sentences, in which the novel words were sentence final) for all novel words and then performing the primed lexical decision task, Borovsky et al.'s participants were given blocks of context sentences and prime-target pairs. This configuration was used to minimise potential differences due to recency of exposure to the novel words and to maximise the priming effects for novel words presented in the beginning of the study, however, it could have increased the participants' awareness of the experimental manipulation. This design also bears some similarity to word-to-word association priming, suggesting that the reported priming effects could have been underpinned by the episodic memory system and thus do not address integration.

Finally, only some of the electrophysiological studies have also examined whether there were behavioural priming effects. Among those studies, both behavioural and electrophysiological correlates of priming were found in all studies using the semantic-relatedness judgement task (Bakker et al., 2015; Balass et al., 2010; Perfetti et al., 2005), while, in studies featuring the primed lexical decision task (Batterink \& Neville, 2011; Borovsky et al., 2012, 2013), no behavioural priming was observed. This is an intriguing dissociation, and, although there are other reports in the literature where either electrophysiological or behavioural semantic priming effects are present (e.g., McLaughlin et al., 2004, in L2 word learning; Chwilla et al., 2000, in mediated priming), the relationship between these effects warrants further investigation. 


\section{Summary}

Integration of novel names for novel concepts into semantic memory has been extensively studied with both behavioural and electrophysiological methods. While this literature has shed light on the process of integration, it has also sparked many questions. For instance, it remains unclear whether novel words can compete with familiar words immediately after or first 24 hours after exposure, and under what conditions. Where lexical competition has been observed, the nature of both the behavioural and electrophysiological priming effects require further investigation. This is because the design of some studies does not allow the disentanglement of word-toword association priming, arising through co-occurrence of primes and targets prior to the administration of the priming task, from priming driven by semantic relatedness. Yet, this is an important distinction, because the CLS account of word learning predicts that associative priming does not require integration; rather, it can be accommodated by episodic memory.

Another reason why the nature of some of the observed priming effects may be unclear is that many studies have explicitly asked participants to consider the relationship between the primes and the targets (in the semantic-relatedness judgement task, for example; see Balass et al., 2010; Bakker et al., 2015; Batterink \& Neville, 2011; Liu \& van Hell, 2020; Perfetti et al., 2005), hence, restricting the extent to which priming can be unequivocally attributed to automatic lexical retrieval processes. The primed lexical decision task alleviates these concerns but, in its most common form, where only targets are judged, it is also subject to strategic and conscious processing as the participants are always aware of the fact that the words are paired (e.g., Hutchison, 2003; Lucas, 2000; McRae \& Boisvert, 1998). To date, only one (behavioural) study (Tamminen \& Gaskell, 2013) has used a version of this task that is less prone to strategic influences (in their case, masked primed lexical decision). However, some authors have argued that, to minimise the influence of both prospective and retrospective strategic processes (e.g., de Mornay Davies, 1998; McNamara \& Altarriba, 1988; McRae \& Boisvert, 1998, but see Shelton \& Martin, 1992, for the intra-lexical priming hypothesis), it is necessary to use the continuous primed lexical decision task, where lexical decision is performed on both primes and targets.

\section{Present study}

The aim of the present study was to examine how behavioural markers of integration map onto electrophysiological markers of integration when young adults learn novel names for novel 
concepts. Following the previous studies in the literature, we used lexical facilitation in word recognition as an index of integration. We taught our participants two sets of novel names, one set on each of two consecutive days (remote and recent set, respectively), and then studied whether these novel names facilitated recognition of semantically related familiar words. This was assessed by measuring behavioural and electrophysiological priming effects between the novel names and the familiar words immediately after (the recent set) and 24 hours after (the remote set) exposure to the novel names.

Thus, the design of our study was very similar to that of Bakker et al. (2015) and Liu and van Hell (2020), with the exception of a few methodological differences, both in the training and in the testing phase (see Methods). Critically, to diminish the impact of strategic processing on word recognition, in contrast to previous studies, we assessed lexical competition using a continuous primed lexical decision task rather than a paired primed lexical decision or a semantic-relatedness judgement task. Following Bakker et al. (2015) and Liu and van Hell (2020), we used trained novel names as targets and familiar words as primes, however, we did not expose the participants to familiar words as part of training. We wished to investigate whether behavioural (faster response times to related vs. unrelated targets) and/or electrophysiological (reduced N400 and enhanced LPC amplitudes to related vs. unrelated targets) priming effects would emerge for the trained novel name targets and whether these effects would vary depending on the length of the consolidation period (0-day consolidation period for the recent set vs. 1-day consolidation period for the remote set). Furthermore, since enhanced negativity to unknown pseudowords compared to familiar words in the N400 spatiotemporal window has been suggested to reflect the difference in the lexical status (e.g., Bentin, 1987; Kutas \& Federmeier, 2011), we also contrasted the ERP responses to trained novel names with ERP responses to untrained pseudowords and familiar words in this spatiotemporal window. This study was pre-registered, and the pre-registration is publicly available on OSF (https://doi.org/10.17605/OSF.IO/SU7D3), together with the data (behavioural as well as raw and pre-processed EEG data) and the scripts (https://doi.org/10.17605/OSF.IO/YCUKN). 


\section{Methods}

\section{Participants}

\footnotetext{
${ }^{1}$ As we were not able to conduct a power analysis due to lack of information necessary for data simulation (e.g., prior distributions of model parameters and hyperprior distributions for the parameters of the random effects distributions), we did not pre-register a sample size for this study. In addition, while it is possible to determine what sample size is needed to obtain $95 \%$ credible intervals for the parameters of interest that do not contain zero within their bounds (e.g., De Santis \& Gubbiotti, 2021; Gelfand \& Wang, 2002; Spiegelhalter et al., 2003), there is currently no established method to determine a sample size that would allow for model selection. Moreover, some authors have argued that there is no need for pre-planned sample sizes in the Bayesian framework (e.g., Lilford et al., 1995). We did, however, pre-register that all data would be collected within a 6-month time window. Our final sample size $(N=$ 71 ) is considerably larger than that of previous studies with similar designs (e.g., $N=22$ in Bakker et al., 2015 and $N=23$ in Liu \& van Hell, 2020).
} 
order). EEG was recorded throughout Session 2.

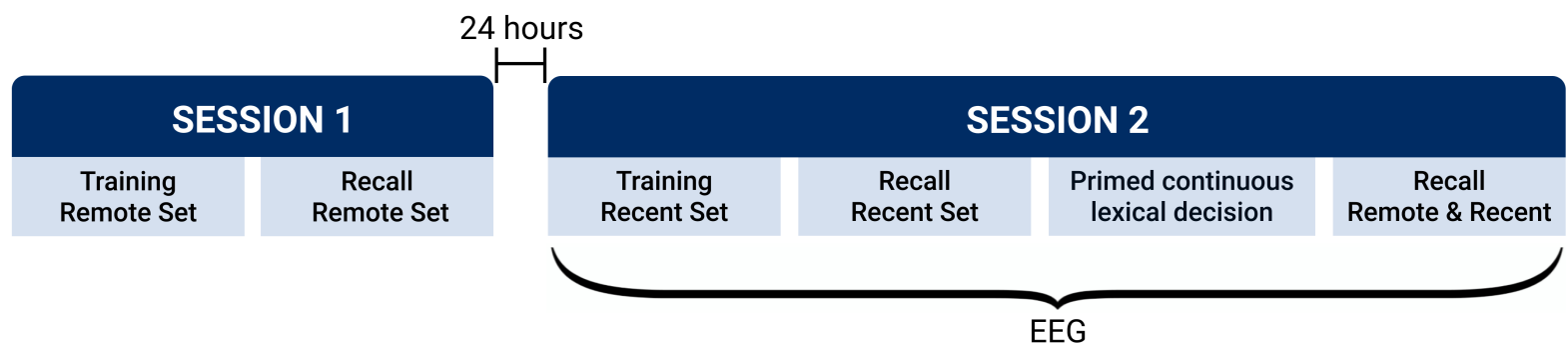

Figure 1. Experimental timeline.

The experiment was run using Presentation software (Version 20.2, Build 07.25.18; Neurobehavioral Systems, Inc., Berkeley, CA, www.neurobs.com) at Macquarie University, Australia. Participants were tested individually in a quiet room. The mean time elapsing between the two sessions was 25.82 hours $(S D=2.80$, range $=16-31.50)$. All participants reported having their usual duration and quality of sleep the night between the two sessions. Session 1 lasted 1.5 hours and Session 2 took 2 hours, including breaks and the EEG set up. Data from the learning phase and the working memory test will be reported elsewhere.

\section{Learning phase}

Participants were required to learn novel names for 40 novel concepts presented with the descriptions of those concepts.

\section{Materials}

Concept descriptions. Forty (existing) concepts were selected, each from a different semantic category (e.g., one plant, one bird, one drink, etc.). From those concepts, 40 novel concepts were derived such that each novel concept was designed to represent a highly similar category coordinate. For example, if aloe was selected as an existing concept, a novel plant was generated that was similar to aloe but with partly different semantic features (e.g., ability to survive extreme drought by curling into a tight ball and uncurling when exposed to water). Each of the 40 novel concepts was described with 4 short sentences that did not include the category names of the concepts (sentence length: $M=9.98$ words, $S D=2.58$ ).

Twenty three Australian native speakers (5 male, age: $M=36.17, S D=13.47$ ) were given these descriptions along with descriptions of 20 existing concepts (fillers). The participants 


\section{A}

It lives in saltwater and has a prominent dorsal fin.

It is able to detect electromagnetic fields that living things produce.

It has a small body and an even smaller head and moves very slowly.

It always swims near the surface.

\section{B}

It has tiny round leaves and very strong roots.

It is only found in deserts.

It can survive extreme drought by curling into a tight ball.

It uncurls when exposed to water.

Table 1. Sentences that formed the concept descriptions of (A) a novel fish similar to a shark, and (B) a novel plant similar to a cactus. 
Novel names. Forty novel words were created, half monosyllabic and half disyllabic. All novel words were phonotactically and orthographically legal in Australian English. Ten native speakers of Australian English judged that these novel words neither existed in English nor elicited strong associations with existing English words. A recording of each novel word was made by a male native speaker of Australian English.

Lists. To ensure that each concept description and each novel name appeared in both the remote and the recent set, 4 lists were created. To do so, we first generated two sets of novel concept descriptions (1 and 2) and two sets of novel names (A and B) by splitting the novel concept descriptions and the novel names into groups of 20. Each set of novel names had an equal number of monosyllabic $(N=10)$ and disyllabic $(N=10)$ novel words, and a Bayesian alternative to a t-test (as implemented in the R packages BEST and BayesFactor; see Appendix A for model output) was applied confirming that the two sets were matched for orthographic Levenshtein distance 20 (OLD20, see Yarkoni et al., 2008; computed using the R package vwr), number of letters and bigram frequency (as given in the MCWord database; Medler \& Binder, 2008). The novel names were then paired with the descriptions to create four sets of pairs with 20 pairs per set: $1 \mathrm{~A}$ - Description Set 1 with Novel name Set A, 1B - Description Set 1 with Novel name Set B, 2A - Description Set 2 with Novel name Set A, 2B - Description Set 2 with Novel name Set B. Four lists were constructed such that there were all possible combinations of sets across the lists: List 1 - set 1A in Session 1 and 2B in Session 2, List $2-1 \mathrm{~B}$ and 2A, List $3-2 \mathrm{~A}$ and $1 \mathrm{~B}$ and List $4-2 \mathrm{~B}$ and $1 \mathrm{~A}$. The participants were randomly assigned to the lists, and there was an equal number of participants $(N=9)$ per list. The concept description-novel name pairs assigned to each list can be seen in Appendix B.

\section{Procedure}

In each session, participants learned one set of 20 novel concept description-novel name pairs (Figure 2). In both sessions, each trial started with a fixation cross shown for $500 \mathrm{~ms}$. Then, the written form of a novel name (in black Arial font, font size 60) appeared in the middle of the screen. The participants were asked to view it silently for $2000 \mathrm{~ms}$, during which a recording of the spoken form of the novel name was played. Next, four sentences describing the novel concept associated with the novel name appeared one by one (in black Arial font, size 25), with 
$3000 \mathrm{~ms}$ between each sentence, with previous sentences remaining on the screen. After the last sentence, all four sentences stayed on the screen for another $9000 \mathrm{~ms}$, together with the novel name. Following a blank screen for $1000 \mathrm{~ms}$, the written form of the novel name (Arial font, size 60) was presented again, this time in red and with an image of a microphone above it, for $3000 \mathrm{~ms}$, and the participants were asked to read the novel name aloud. After a blank screen for $1000 \mathrm{~ms}$, the next trial began.

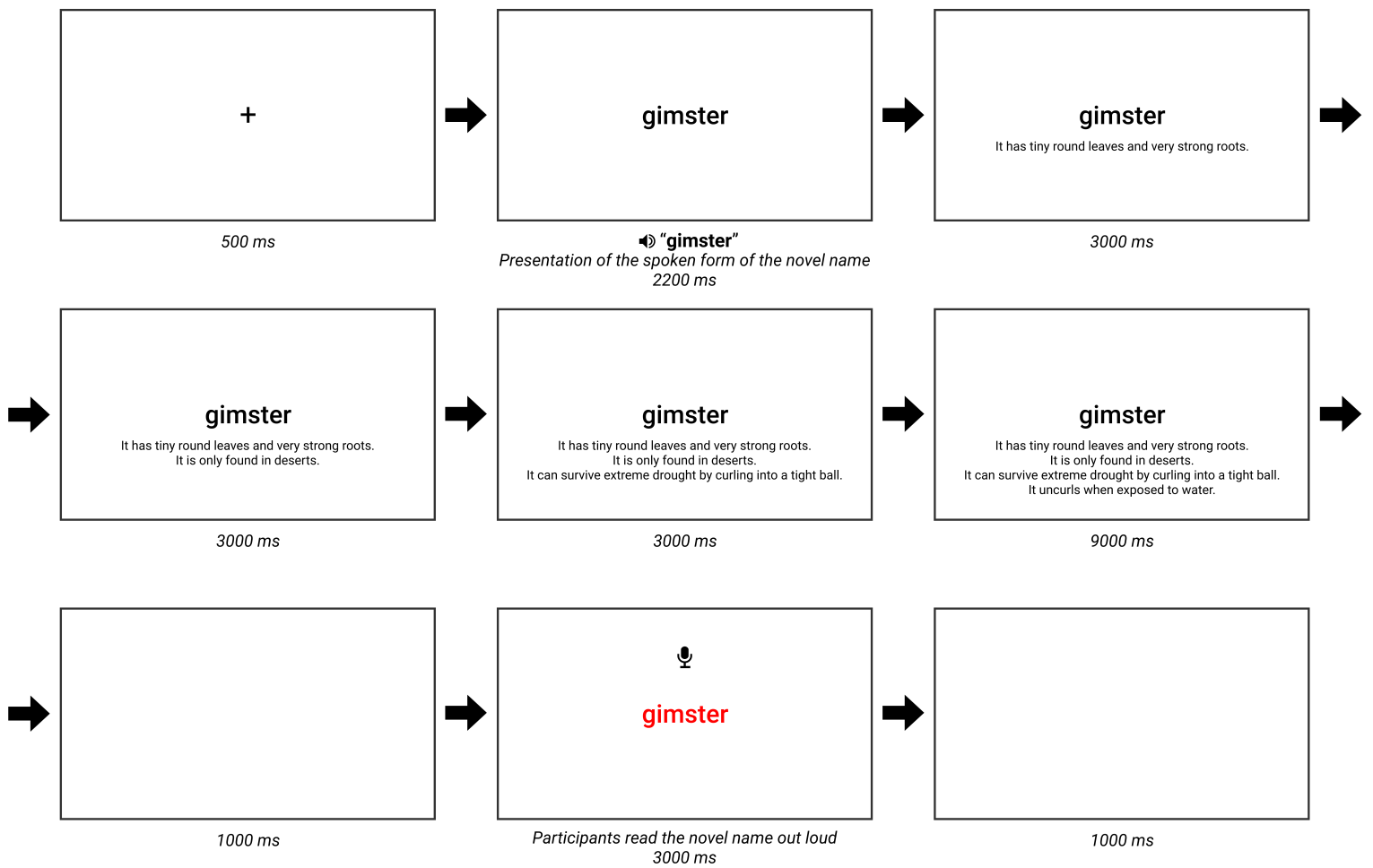

Figure 2. Example of one trial in the learning phase.

When all the novel names and concept descriptions had been presented once, the set of 20 pairs was presented three more times, each time in a different order. The presentation order was pseudo-randomised such that (a) the last novel name shown in one round was not first in the next round and (b) none of the novel names appeared at the same position more than twice across the four rounds. Therefore, the participants were exposed to each novel concept description-novel name pair 4 times. In each session, the learning phase took approximately 45 minutes including breaks. 


\section{Recall tasks}

Immediately following the learning phase, a recall task was used to enable evaluation of learning in each session. In the recall task, participants were presented with the concept descriptions from the set of 20 names they had just learned (i.e., the remote set in Session 1 and the recent set in Session 2) and asked to provide the names of the novel concepts associated with the descriptions. Each trial started with a $500 \mathrm{~ms}$ fixation screen. The descriptions (sets of four sentences) appeared on the screen (in black Arial font, size 25) with an image of a microphone above them. The participants were required to respond orally (with a timeout set to $5000 \mathrm{~ms}$ ) and, after the image of the microphone disappeared, type the written form of the novel name in a response box displayed under the description (with a timeout of $10000 \mathrm{~ms}$ ). The task lasted approximately 5 minutes (including one break).

Participants completed another recall task at the end of Session 2 (following the primed continuous lexical decision task, see below). The procedure was identical to that described above, however, descriptions of all novel concepts (from both the remote and the recent set) were presented. The descriptions appeared in a random order (i.e., not blocked by set). The task lasted approximately 10 minutes including breaks.

By administering single-set word recall tasks immediately after a corresponding training phase we sought to ensure that both sets had an equal amount and type of exposure prior to the administration of the priming task. These tasks also gave us a measure of explicit learning (number of words recalled correctly). The second recall task (both sets) in Session 2 was used to (a) examine explicit memory for words from the recent set after a 1-day consolidation period and (b) assess whether the priming task has benefitted explicit memory for the remote set.

\section{Primed continuous lexical decision task}

The design of this task was based on McRae and Boisvert (1998, Experiment 1).

\section{Materials}

Eighty word pairs, each consisting of a prime word and a target word, were created. Half of the word pairs $(N=40)$ comprised pairs of English words (English pairs), while the other half $(N=40)$ consisted of an English prime word and a novel target name from the learning phase 
(English-novel pairs). For both English and English-novel pairs, there were 20 semantically related pairs (with primes and targets belonging to the same semantic category; semantically related condition) and 20 semantically unrelated pairs (primes and targets from different semantic categories; semantically unrelated condition). The full list of stimuli used in the primed continuous lexical decision task is provided in Appendix C.

English-novel pairs. For each of the 40 trained novel names, a semantically related English word and a semantically unrelated English word were selected as primes. The semantically related primes comprised the base English words from which the novel concepts were derived (e.g., cactus for the novel concept shown in Table 1$)$. The semantically unrelated pairs $(N=$ 40) were created by pseudo-randomly re-pairing the primes and the targets to ensure that words from different but semantically close categories (e.g., animals and birds, or food and drinks) were not paired as unrelated.

English pairs. We selected one pair of familiar concepts from each of the semantic categories $(N=40)$ used in the English-novel pairs. We aimed to ensure that the category coordinates were as similar as possible. However, to minimise the 'associative boost' observed when a semantic relationship is accompanied by a normative association (e.g., Lucas, 2000), we ensured a low associative relationship (the two words were not among the first three of each other's associates and had the associative strength of 0.1 or less as given in the Small World of Words association norms; De Deyne et al., 2018). As with the English-novel pairs, the semantically unrelated English pairs $(N=40)$ were created by pseudo-randomly re-pairing the primes and the targets. Fifteen Australian native speakers (2 male, age: $M=27.87, S D=8.78$ ) then judged the semantic relatedness of the pairs by specifying whether the pairs of words referred to the same concept. If they responded negatively, they were asked to rate how similar in meaning the words in the pairs were to each other. A 7-point scale was used, where 1 stood for 'very different' and 7 for 'very similar'. The mean rating of the related pairs was $5.26(S D=1.68)$ and that of the unrelated pairs was $1.28(S D=0.97)$.

Fillers. In addition to the experimental pairs, each list included 160 filler nonwords to equalize the number of 'yes' and 'no' responses for the lexical decision. These nonwords were created following the same criteria as for the novel names used in the learning phase (equal number 


\begin{tabular}{|c|c|c|}
\hline & Semantically related & Semantically unrelated \\
\hline $\begin{array}{l}\text { English pairs } \\
(N=40)\end{array}$ & $\begin{array}{l}20 \text { pairs with targets from } \\
\text { semantic categories } 1-20\end{array}$ & $\begin{array}{l}20 \text { pairs with targets from } \\
\text { semantic categories } 21-40\end{array}$ \\
\hline \multirow{2}{*}{$\begin{array}{l}\text { English-novel } \\
\text { pairs }(N=40)\end{array}$} & $\begin{array}{l}10 \text { pairs from Set } 1 \text { with targets } \\
\text { from semantic categories } 21-30\end{array}$ & $\begin{array}{l}10 \text { pairs from Set } 1 \text { with targets } \\
\text { from semantic categories } 1-10\end{array}$ \\
\hline & $\begin{array}{l}10 \text { pairs from Set } 2 \text { with targets } \\
\text { from semantic categories } 31-40\end{array}$ & $\begin{array}{l}10 \text { pairs from Set } 2 \text { with targets } \\
\text { from semantic categories } 11-20\end{array}$ \\
\hline
\end{tabular}

Table 2. Experimental stimuli in one of the lists used in the primed continuous lexical decision task. 


\section{Procedure}

Both experimental (primes and targets) and filler stimuli were presented to the participants in lowercase (in black Arial font, size 60), one stimulus at a time. For each letter string, the participants were required to judge whether it corresponded to a word that they knew by pressing an appropriate button (the top left button for 'yes' and the top right button for 'no' on a Cedrus RB-840 button box). The participants were explicitly instructed to respond 'yes' to both familiar (existing) English words and trained novel names from both sets.

Primes and targets were not paired explicitly but appeared as adjacent items, with lexical decision being required for every item. The presentation order was pseudo-randomised separately for each participant with the following constraints: (a) a maximum of four consecutive items per condition (related, unrelated, filler), (b) a maximum of four consecutive items where the novel names (targets) were from the same set (remote or recent). This design and the low proportion of semantically related pairs $(25 \%)$ was used to minimise strategic processing and awareness of the experimental manipulation by the participants.

The task started with 20 practice trials. The experimental trials were presented in blocks of 20, with short breaks in between. Each block started with a filler trial. The participants were told to respond as quickly and as accurately as possible upon the presentation of the stimulus. Independent of whether and when a response was given, the stimulus stayed on the screen for $1000 \mathrm{~ms}$ (timeout). This was followed by a blank screen for $200 \mathrm{~ms}$. The blank screen was replaced by the next stimulus, either experimental or filler.

\section{Pre-processing of behavioural data}

Recall tasks. The accuracy of the participants' vocal responses was manually checked using Praat (Boersma \& Weenink, 2014), and only responses that corresponded to the trained phonological form were counted as correct. The judge was blind to the experimental condition in which the responses were collected. The accuracy of the typed responses was evaluated automatically by the Presentation software and only responses that matched the trained orthographic form were coded as correct.

Primed continuous lexical decision task. Prior to conducting any descriptive or inferential statistical analysis, we inspected the distribution of the response times across 
participants and items. Data points that clearly stood outside the distribution were removed (Baayen, 2008). This resulted in less than 1 per cent data loss.

\section{Acquisition and pre-processing of electrophysiological data}

EEG was recorded using a 64-channel BioSemi ActiveTwo electrode system (Amsterdam, Netherlands) at a sampling rate of $2048 \mathrm{~Hz}$. The Ag-AgCl-tipped electrodes were attached to an electrode cap using the 10/20 system. The signal was recorded relative to an additional (active) electrode, the common mode sense (CMS), which formed a feedback loop with another additional (passive) electrode, the driven right leg (DRL). These two electrodes replaced the ground electrode of more conventional systems. Further electrodes were placed on the left and right mastoid as well as at the outer canthus of the left eye. The raw EEG signal was pre-processed in MATLAB (version R2020b; Natick, Massachusetts: The MathWorks Inc), using the EEGLAB toolbox (Delorme \& Makeig, 2014). Two datasets were created. For both datasets, the continuous signal was re-sampled at $500 \mathrm{~Hz}$ and filtered with a $1 \mathrm{~Hz}$ high pass filter (Kaiser windowed sinc FIR filter, order $=802$, beta $=4.9898$, transition bandwidth $=$ $2 \mathrm{~Hz}$ ) and a $40 \mathrm{~Hz}$ low pass filter (Kaiser windowed sinc FIR filter, order $=162$, beta $=4.9898$, transition bandwidth $=10 \mathrm{~Hz}$ ). Line noise removal and bad channel detection were performed using the cleanLineNoise and findNoisyChannels functions from the PREP pre-processing pipeline (Bigdely-Shamlo et al., 2015), and both datasets were re-referenced to the algebraic average of the left and right mastoid. Subsequently, one of the datasets (excluding the bad channels) was subjected to Independent Component Analysis (ICA; Chaumon et al., 2015), while the other dataset was high pass filtered at $0.1 \mathrm{~Hz}$ (Kaiser windowed sinc FIR filter, order $=8008$, beta $=4.9898$, transition bandwidth $=0.2 \mathrm{~Hz})$.

The demixing matrix obtained from the ICA (run on dataset 1) was then applied to the second dataset (filtered at $0.1 \mathrm{~Hz}$ ). ICLabel, an automated EEG independent component classifier (Pion-Tonachini et al., 2019), was used to exclude individual components corresponding to activity originating from the eyes and groups of muscle motor units as well as the electrocardiographic signals. Next, the noisy channels identified earlier were spherically interpolated. The signal was then segmented into epochs of $1.2 \mathrm{~s}$, from $200 \mathrm{~ms}$ before the stimulus onset to $1 \mathrm{~s}$ after the stimulus onset. The epochs were baseline corrected using the mean of the signals between $-200 \mathrm{~ms}$ and $0 \mathrm{~ms}$ relative to the stimulus onset. Epochs with the 
amplitude below $-100 \mu \mathrm{V}$ or above $100 \mu \mathrm{V}$ were excluded. At the end of the pre-processing, a mean of 3.73 epochs per participant $(S D=9.30$, range $=0-62)$ were excluded (note that epochs corresponding to the primes were not analysed). In the semantic priming analysis for the English pairs, a mean of 0.32 epochs per participant per condition (related: $S D=0.89$, range $=0-5$; unrelated: $S D=0.95$, range $=0-5)$ were excluded. In the semantic priming analysis for the English-novel pairs, a mean of 0.20 epochs per participant were removed for the recent set $(S D=0.69$, range $=0-5$; related: $M=0.20, S D=0.62$, range $=0-4$; unrelated: $M=0.21, S D=0.75$, range $=0-5)$ and 0.12 epochs per participant for the remote set $(S D=0.40$, range $=0-3$; related: $M=0.10, S D=0.34$, range $=0-2$; unrelated: $M=$ $0.14, S D=0.46$, range $=0-3)$. For the filler nonwords in the lexicality analysis, a mean of 2.44 epochs per participant $(S D=5.98$, range $=0-41)$ were discarded. Finally, epochs corresponding to trials with incorrect behavioural responses to the target words were removed, which, together with the epochs excluded earlier, constituted $13.7 \%$ of the data. As with the behavioural data, prior to conducting any descriptive or inferential statistical analysis, we inspected the distribution of the amplitudes across participants and items, and one additional data point (in the dataset restricted to the novel name targets, see Main analysis) was removed.

\section{Analysis}

For all tasks, standard errors and confidence intervals were computed using the method proposed in Morey (2008) that takes into account within-subjects variability in within-subject designs (implemented in the R package Rmisc).

All Bayesian models (behavioural and electrophysiological, see below) were performed in the statistical software R, version 4.1.1 (R Core Team, 2021) using the brms package (Bürkner, 2017a, 2017b). For each model, four sampling chains were run, each with 10000 iterations. Of the 10000 iterations, the initial 2000 were warm-up iterations. Thus, there were a total of 32000 sampling iterations available for analysis. The Bayesian model output provides a posterior distribution of possible values for a parameter given the data and the model. We report the estimate of the posterior mean with its $95 \%$ credible interval (95\% CrI), which denotes the range of values within which we can be $95 \%$ certain that the true value of the parameter falls (e.g., 
Nicenboim \& Vasishth, 2016).

In the Bayesian analyses, hypothesis testing used Bayes factors (e.g., Jeffreys, 1939; Heck et al., 2020; Kass \& Raftery, 1995; Schönbrodt \& Wagenmakers, 2018) via the bridgesampling algorithm (Bennett, 1976; Meng \& Wong, 1996; Schad, Nicenboim, Betancourt, \& Vasishth, 2021; Schad, Nicenboim, Bürkner, et al., 2021) as implemented in the $\mathrm{R}$ package bridgesampling (Gronau et al., 2020). Following the traditional notation, we denote Bayes factors representing the ratio of the likelihood of a more complex hypothesis to the likelihood of a simpler hypothesis as $B F_{10}$ and interpret the strength of change of evidence in favour of one model over another using the Bayes factor scale proposed in Jeffreys (1939). According to this scale, $B F_{10}>1$ provides evidence for a more complex model $\left(1<B F_{10}<3\right.$ : anecdotal evidence, $3<B F_{10}<10$ : moderate evidence, $10<B F_{10}<30$ : strong evidence, $30<B F_{10}<100$ : very strong evidence, $B F_{10}>100$ : extreme evidence), $B F_{10}=1$ provides no evidence, and $B F_{10}<1$ provides evidence for a less complex model $\left(\frac{1}{3}<B F_{10}<1\right.$ : anecdotal evidence, $\frac{1}{10}<B F_{10}<\frac{1}{3}$ : moderate evidence, $\frac{1}{30}<B F_{10}<\frac{1}{10}$ : strong evidence, $\frac{1}{100}<B F_{10}<\frac{1}{30}$ : very strong evidence, $B F_{10}<\frac{1}{100}$ : extreme evidence).

Apart from the packages listed above and in the Methods section, the following $\mathrm{R}$ packages were used: openxlsx, tidyverse, lme4, MASS, scales, rstan, rethinking, designr, reshape, RColorBrewer, gridExtra, bayesplot, EnvStats, cowplot.

In addition to analyses described in this section, we pre-registered another analysis to examine whether the process of integration is modulated by individual differences in working memory capacity (see Pre-registration). This analysis has not been performed yet and will be reported elsewhere.

\section{Behavioural data}

Behavioural data (response times for correct responses) was analysed using Bayesian Linear Mixed Effects models (e.g., Nicenboim \& Vasishth, 2016). Two analyses were pre-registered: the preliminary analysis examined whether there was a semantic priming effect for the English targets, while the main analysis examined (a) whether there was a semantic priming effect for the trained novel name targets and (b) whether this effect was modulated by the length of the consolidation period (1-day consolidation for the remote set, 0-day consolidation for the recent set). There were no deviations from the pre-registered analyses. 
All models included the maximal random effects structure for the location parameter $\mu$ (i.e., correlated varying intercepts and slopes for subjects and items, see e.g., Barr et al., 2013). The contrasts of interest were defined using sum contrast coding $(-1,+1)$. All models were fit with three sets of priors (as part of a sensitivity analysis) that differed in terms of their informativeness about the effect of interest (diffuse, weakly informative, strongly informative). In all models, the correlation matrix for the random effects was defined with an LKJ-prior (Lewandowski et al., 2009; Stan Development Team, 2017) with a diffuse parameter value 2. This prior allows regularisation of the distributions of the correlation parameters such that extreme values such as +1 and -1 are less likely a priori. All other model parameters were specified using the log-normal distribution (see Table 3). In all analyses, for the intercept, we assumed a normal distribution with a mean of 6.5 and a standard deviation of 0.5 , reflecting our a priori expectation that response times were an average of $\exp (6.5)=665 \mathrm{~ms}$ with $95 \%$ of values lying between $\exp (6.5-2 \times 0.5)=245 \mathrm{~ms}$ and $\exp (6.5+2 \times 0.5)=1808 \mathrm{~ms}$. This prior was based on average response times that are typically reported in the literature in the (paired and continuous) primed lexical decision task as well as our expectation that mean lexical decision times would be similar for familiar words and trained novel names. The prior on the effect size on a log scale specifies an effect size which is a multiplicative factor. Therefore, the priors (and hence, the predictions) for the effects of interest depended on the intercept. For example, for the effect of condition (unrelated - related), with diffuse priors, we assumed a normal distribution with a mean of 0 and a standard deviation of 0.05 . For an intercept of $665 \mathrm{~ms}$, this means that we are $95 \%$ certain that the effect of condition would lie between $\exp (\log (665)-2 \times 0.1)-665)=$ $-121 \mathrm{~ms}$ and $\exp (\log (665)+2 \times 0.1)-665=147 \mathrm{~ms}$. In contrast, more informative priors encoded our a priori assumption that the response times in the unrelated condition should be, on average, $\exp (6.5+0.02)-\exp (6.5-0.02)=27$ ms longer than in the related condition. In the following section, we report the back-transformed model estimates as milliseconds for ease of interpretation.

\section{Electrophysiological data}

Electrophysiological data was analysed with Bayesian Distributional Regression models (e.g., Rigby \& Stasinopoulos, 2005) and Mass Univariate analysis (e.g., Groppe et al., 2011a, 2011b). Two analyses were pre-registered: the preliminary analysis examined whether there were 


\begin{tabular}{|c|c|c|c|c|c|}
\hline & & & Diffuse & $\begin{array}{l}\text { Weakly } \\
\text { informative }\end{array}$ & $\begin{array}{l}\text { Strongly } \\
\text { informative }\end{array}$ \\
\hline \multirow{6}{*}{$\begin{array}{l}\text { Main } \\
\text { analysis }\end{array}$} & \multirow{4}{*}{$\begin{array}{l}\text { Preliminary } \\
\text { analysis }\end{array}$} & Intercept & $\mathcal{N}(6.5,0.5)$ & $\mathcal{N}(6.5,0.5)$ & $\mathcal{N}(6.5,0.5)$ \\
\hline & & Condition & $\mathcal{N}(0,0.05)$ & $\mathcal{N}(0.02,0.008)$ & $\mathcal{N}(0.02,0.004)$ \\
\hline & & $S D$ & $\mathcal{N}(0,0.1)$ & $\mathcal{N}(0,0.05)$ & $\mathcal{N}(0,0.05)$ \\
\hline & & $\sigma$ & $\mathcal{N}(0,0.5)$ & $\mathcal{N}(0,0.5)$ & $\mathcal{N}(0,0.5)$ \\
\hline & & Set & $\mathcal{N}(0,0.05)$ & $\mathcal{N}(-0.02,0.008)$ & $\mathcal{N}(-0.02,0.004)$ \\
\hline & & Condition $\times$ Set & $\mathcal{N}(0,0.05)$ & $\mathcal{N}(-0.02,0.008)$ & $\mathcal{N}(-0.02,0.004)$ \\
\hline
\end{tabular}

Table 3. Priors used in the analysis of the behavioural data (log scale).

differences in averaged amplitudes between the semantically related and unrelated English targets in the N400 and in the LPC spatiotemporal windows. The main analysis examined (a) whether there were differences in averaged amplitudes between the semantically related and unrelated trained novel name targets in the N400 and in the LPC spatiotemporal windows, (b) whether this effect was modulated by the length of the consolidation period (0-day consolidation for the recent set and 1-day consolidation for the remote set; henceforth, priming analysis), and (c) whether there were differences in averaged amplitudes between the semantically unrelated English targets, the semantically unrelated trained novel name targets and the nonwords (henceforth, lexicality analysis).

Analysis of mean amplitudes in two pre-defined spatiotemporal windows. The dependent variable was amplitude averaged across all time points and electrodes within the pre-defined spatiotemporal windows, the N400 and the LPC. We selected 23 centro-parietal electrodes (C5, C3, C1, Cz, C2, C4, C6, CP5, CP3, CP1, CPz, CP2, CP4, CP6, P7, P5, P3, P1, Pz, P2, P4, P6, P8) between $300 \mathrm{~ms}$ and $500 \mathrm{~ms}$ after word onset for the N400 spatiotemporal window, while the LPC spatiotemporal window spanned 9 frontal (F7, F5, F3, F1, Fz, F2, F4, F6, F8) and 9 parietal (P7, P5, P3, P1, Pz, P2, P4, P6, P8) electrodes between $500 \mathrm{~ms}$ and $800 \mathrm{~ms}$ post onset. This choice was based on which channels are typically selected in the literature (for the N400 see, e.g., Cheimariou et al., 2018; Liu \& van Hell, 2020; Nieuwland et al., 2019; Payne et al., 2015; Szewczyk \& Schriefers, 2018; and for the LPC, e.g., Bakker et al., 2015; Batterink \& Neville, 2011; Peters \& Daum, 2009; Rugg \& Curran, 2007). The frontal electrodes were included in the LPC spatiotemporal window because it has been suggested that electrophysiological correlates of recollection differ depending upon the type of information that must be retrieved from episodic memory (e.g., Peters \& Daum, 2009). 
In line with the pre-registered plan, we first fitted Linear Mixed Effects models with a maximal random effects structure for the location parameter $\mu$, however, these models misfitted the by-subject standard deviations. We, therefore, used Distributional Regression models with a group-level random effects structure for both the location and the scale $(\sigma)$ parameters, and these models provided a much better fit for our data. All models included varying intercepts for the scale parameter, while, for the location parameter, models testing for priming effects included correlated varying intercepts and slopes for subjects and items, and models testing for lexicality effects contained correlated varying intercepts and slopes for subjects and varying intercepts for items (because each item could be either an English word, or a novel name or a nonword, but never all). In the priming analysis, sum contrast coding was used to define the contrasts of interest, while, in the lexicality analysis, we employed repeated contrasts (nonwords vs. English words, recent novel names vs. nonwords, remote vs. recent novel names). There were two more deviations from the pre-registered plan: (1) we fitted separate models for frontal and parietal electrodes in the $500-800 \mathrm{~ms}$ time window to avoid estimation of a three-way interaction, and (2) we used repeated contrasts in the lexicality analysis because the contrasts specified in the pre-registration turned out to be unsuitable for our data.

As with the behavioural data, three sets of priors were defined (diffuse, weakly informative, strongly informative), with the correlation matrices specified using the LKJ-prior. Priors for all other model parameters were defined using the normal distribution (see Table 4). Note that we set the prior on the intercept of sigma to be normally distributed with a mean of 0 and a standard deviation from the log-normal distribution to ensure that negative by-subject adjustments to the intercept did not cause sigma to become negative.

\begin{tabular}{llll}
\hline & Diffuse & $\begin{array}{l}\text { Weakly } \\
\text { informative }\end{array}$ & $\begin{array}{l}\text { Strongly } \\
\text { informative }\end{array}$ \\
\hline Intercept & $\mathcal{N}(0,10)$ & $\mathcal{N}(0,5)$ & $\mathcal{N}(0,5)$ \\
Effect of interest & $\mathcal{N}(0,10)$ & $\mathcal{N}(0,5)$ & $\mathcal{N}(0,2.5)$ \\
$S D$ & $\mathcal{N}(0,20)$ & $\mathcal{N}(0,5)$ & $\mathcal{N}(0,5)$ \\
Intercept for $\sigma$ & $\mathcal{N}(0, \log (50))$ & $\mathcal{N}(0, \log (10))$ & $\mathcal{N}(0, \log (10))$ \\
$S D$ for $\sigma$ & $\mathcal{N}(0,5)$ & $\mathcal{N}(0,5)$ & $\mathcal{N}(0,5)$ \\
\hline
\end{tabular}

Table 4. Priors used in the Bayesian analysis of the electrophysiological data. 
Mass univariate analysis. This analysis was employed to explore time windows and regions other that the N400 and the LPC spatiotemporal windows as the mass univariate analysis allows testing for significant differences between the conditions of interest across the whole scalp and at every time point (see, e.g., Groppe et al., 2011a, 2011b, for details). These analyses were conducted using LIMO, an open-source MATLAB toolbox (Pernet et al., 2011). In LIMO, the data is analysed using a Hierarchical General Linear model. First, model parameters (i.e., beta coefficients for the predictor of interest) are estimated separately for each participant at each time point and electrode (first-level analysis; see Bellec et al., 2012). Next, the parameter estimates are used to test the statistical significance of the predictors at a group level (second-level analysis), and correction for multiple comparisons is applied (via bootstrapping). In the present study, parameter estimates for the first-level analysis were obtained using trial based Ordinary Least Squares, and, for the second-level analysis, by performing robust paired t-tests at each time point and electrode. We corrected for multiple comparisons using the Threshold Free Cluster Enhancement technique (TFCE; see, e.g., Pernet et al., 2015; Smith \& Nichols, 2009). This method was chosen because it avoids some of the known issues of other cluster-based correction approaches (e.g., high sensitivity to the initial cluster-forming threshold), while it provides an equally strong control of the family-wise error rate. There were two deviations from the pre-registered analysis: (1) we used the same contrast coding as in the Bayesian analysis (repeated contrasts) because the pre-registered contrasts proved to be inappropriate for the data, and, (2) while correcting for multiple comparisons, we relied on bootstrapping rather than on permutation tests because bootstrap techniques have been argued to be more versatile (e.g., Pernet et al., 2011; Wilcox, 2005).

\section{Results}

\section{Behavioural data}

\section{Recall tasks}

In the recall task for the remote set, mean response accuracy, for the spoken responses, was $42 \%(S D=13 \%)$ and, for the typed responses, $51 \%(S D=12 \%)$. Mean accuracy in the recall

task for the recent set was very similar, with $44 \%$ correct $(S D=12 \%)$ for the spoken and $51 \%$ 
correct $(S D=13 \%)$ for the typed responses. The seemingly higher accuracy for the typed responses is probably due to the fact that those were given after the spoken responses such that the participants had more time for retrieval. In the recall task for both sets, mean accuracy, for the recent set, was $43 \%(S D=12 \%)$ for the spoken and $49 \%(S D=12 \%)$ for the typed responses, and, for the remote set, $30 \%(S D=14 \%)$ and $34 \%(S D=12 \%)$.

\section{Primed continuous lexical decision task}

\section{Preliminary analysis (English prime — English target)}

The accuracy for the English targets in the related condition (related English targets) was 93\% $(S D=0.07 \%)$ and, for the English targets in the unrelated condition (unrelated English targets), $90 \%$ ( $S D=0.07 \%$ ), and mean response times (for correct responses) in both conditions are shown in Figure 3. The models included only one predictor, condition (unrelated vs. related), and Table 5 displays the results of the analyses with all three sets of priors. The Bayes factors indicated extreme evidence for the alternative model compared to the null model, reflecting faster responses in the semantically related than in the unrelated condition.

\begin{tabular}{lccr}
\hline Prior on the effect of condition & Estimate $(\mathrm{ms})$ & $\mathbf{9 5 \%}$ CrI & $\boldsymbol{B F}_{\mathbf{1 0}}$ \\
\hline Diffuse & 20.90 & {$[12.08,29.35]$} & 1016.37 \\
Weakly informative & 21.14 & {$[13.65,28.65]$} & 6186.23 \\
Strongly informative & 21.21 & {$[15.30,27.15]$} & 9567.63 \\
\hline
\end{tabular}

Table 5. Sensitivity analysis, showing the estimates and the $95 \%$ credible intervals for the behavioural priming effect with the English targets as well as the Bayes factors for the analyses with different priors. 


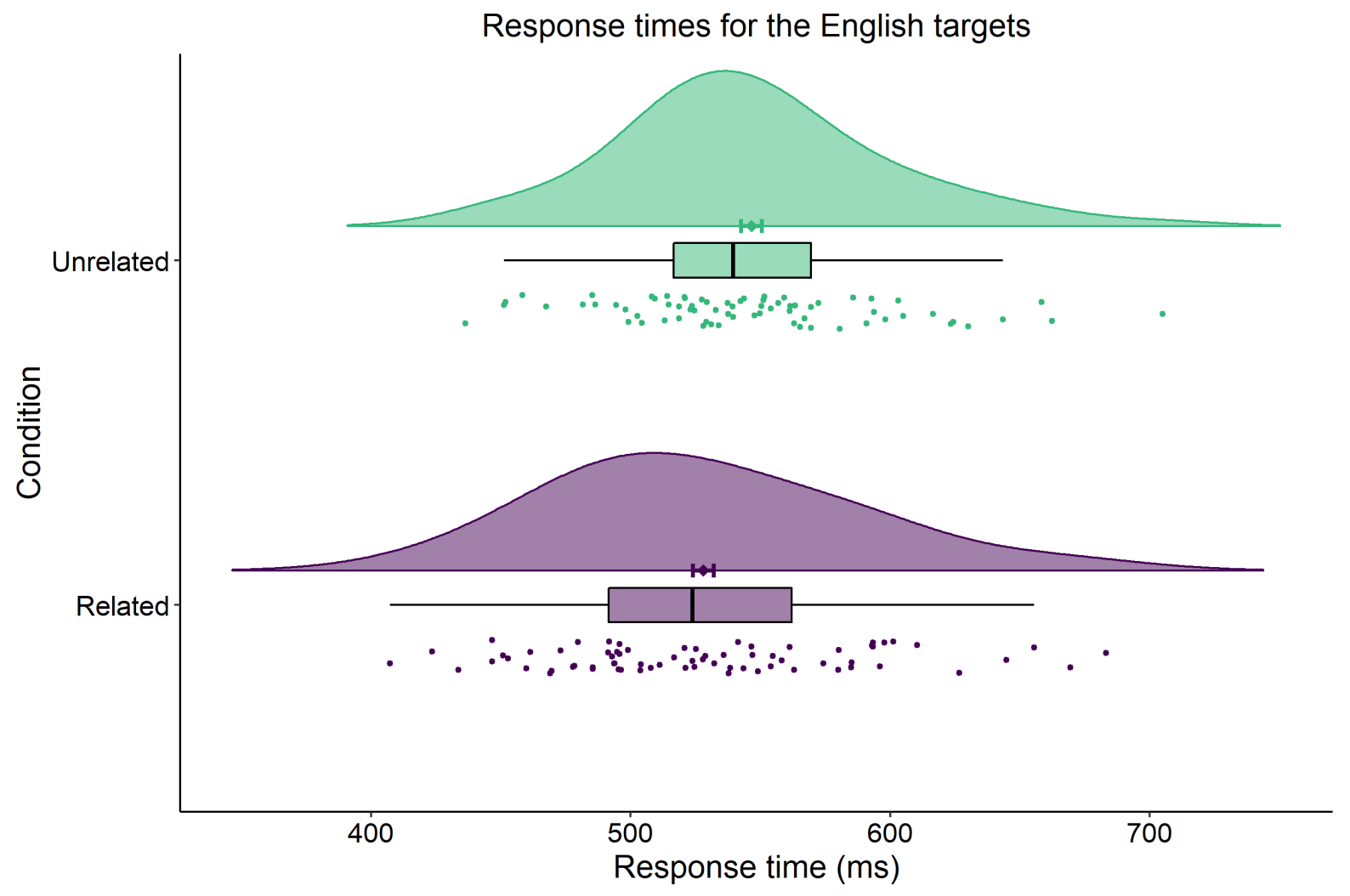

Figure 3. Probability density, medians, means and $95 \%$ confidence intervals for the response times for the semantically related and unrelated English targets. Error bars represent one standard error from the mean.

\section{Main analysis (English prime — Novel name target)}

For the novel name targets trained in the recent set, the participants responded accurately to $89 \%(S D=0.10 \%)$ of the names that appeared in the related condition and to $90 \%$ (SD $=$ $0.10 \%$ ) of the names that were shown in the unrelated condition. For the novel name targets trained in the remote set, $86 \%(S D=0.11 \%)$ of the related targets and $87 \%(S D=0.12 \%)$ of the unrelated targets were responded to correctly. Figure 4 displays mean response times (for correct responses) for the novel name targets depending on whether they followed semantically related or unrelated primes and whether they were trained in the recent or the remote set. The models included two predictors, condition (unrelated vs. related) and set (recent vs. remote), and their interaction. The results of the analyses with all three sets of priors are shown in Table 6 . The Bayes factors indicated moderate to very strong evidence against a main effect of condition, anecdotal evidence in favour of a main effect of set and moderate to very strong evidence against the interaction, suggesting longer response times to targets trained in the remote as compared to the recent set. 


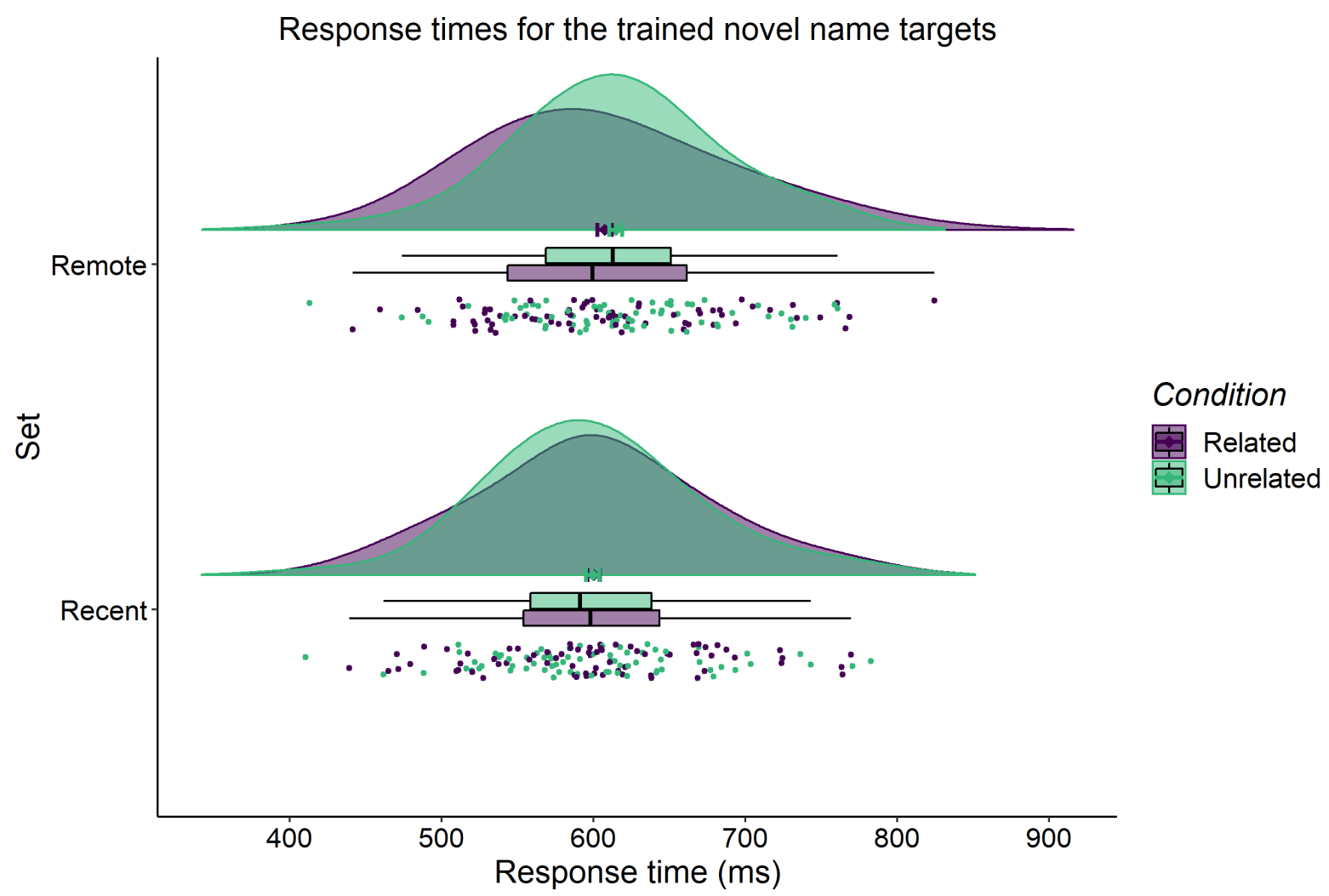

Figure 4. Probability density, medians, means and $95 \%$ confidence intervals for the semantically related and unrelated novel name targets trained in the recent vs. in the remote set. Error bars represent one standard error from the mean.

\begin{tabular}{lcccr}
\hline Prior & Effect & Estimate $(\mathrm{ms})$ & $\mathbf{9 5 \%}$ CrI & $\boldsymbol{B F}_{\mathbf{1 0}}$ \\
\hline \multirow{3}{*}{ Diffuse } & Condition & 4.05 & {$[-5.75,13.89]$} & 0.14 \\
& Set & -11.48 & {$[-21.08,-1.92]$} & 1.42 \\
& Condition $\times$ Set & -4.19 & {$[-12.89,4.45]$} & 0.09 \\
\hline \multirow{2}{*}{ Weakly } & Condition & 8.43 & {$[-0.26,17.41]$} & 0.12 \\
informative & Set & -14.01 & {$[-22.57,-5.60]$} & 3.95 \\
& Condition $\times$ Set & -7.82 & {$[-15.93,0.16]$} & 0.13 \\
\hline \multirow{2}{*}{ Strongly } & Condition & 14.93 & {$[7.75,22.50]$} & 0.02 \\
informative & Set & -17.67 & {$[-24.48,-10.98]$} & 3.29 \\
& Condition $\times$ Set & -13.70 & {$[-20.51,-7.19]$} & 0.01 \\
\hline
\end{tabular}

Table 6. Sensitivity analysis, showing the estimates and the $95 \%$ credible intervals for the behavioural priming effects with the trained novel name targets as well as the Bayes factors for the analyses with different priors. 


\section{Electrophysiological data}

\section{Preliminary analysis (English prime — English target)}

\section{Analysis of mean amplitudes in two pre-defined spatiotemporal windows}

N400 spatiotemporal window. Averaged amplitudes for semantically related and unrelated English targets at centro-parietal electrodes are shown in Figure 5. The models included one predictor, condition, and Table 7 displays the results of the analyses with all three sets of priors. The Bayes factors indicated extreme evidence for the alternative model compared to the null model, reflecting more negative amplitudes in the unrelated than in the related condition.

Mean amplitudes for the English targets at centro-parietal electrodes

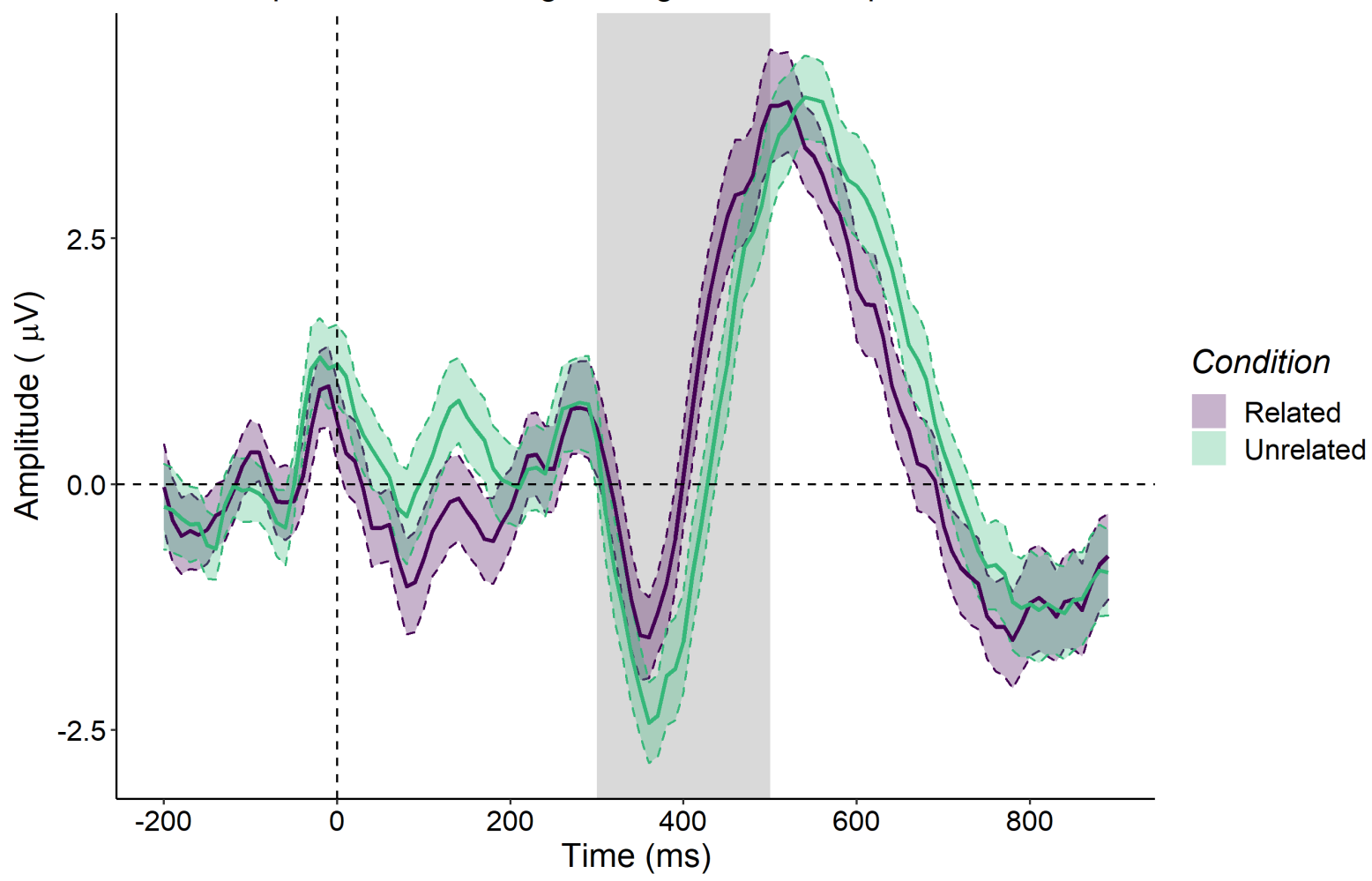

Figure 5. Mean amplitudes (with 95\% confidence intervals) for the semantically related and unrelated English targets at centro-parietal electrodes. The N400 time window is shaded gray.

LPC spatiotemporal window. Figure 6 displays averaged amplitudes for the semantically related and unrelated English targets at the frontal and parietal electrodes. The models contained two predictors, condition (unrelated vs. related) and region of interest (ROI; 


\begin{tabular}{lccc}
\hline Prior on the effect of condition & Estimate $(\mu \mathrm{V})$ & $\mathbf{9 5 \%} \mathbf{C r I}$ & $\boldsymbol{B F}_{\mathbf{1 0}}$ \\
\hline Diffuse & -0.52 & {$[-0.73,-0.30]$} & 173.62 \\
Weakly informative & -0.51 & {$[-0.73,-0.30]$} & 383.62 \\
Strongly informative & -0.52 & {$[-0.73,-0.30]$} & 747.45 \\
\hline
\end{tabular}

Table 7. Sensitivity analysis, showing the estimates and the $95 \%$ credible intervals for the N400 priming effect with the English targets as well as the Bayes factors for the analyses with different priors.

parietal vs. frontal), and their interaction. The results of the analyses with all three sets of

priors are shown in Table 8. The Bayes factors indicated moderate to anecdotal evidence against a main effect of condition, extremely strong evidence in favour of a main effect of ROI, reflecting more positive amplitudes at the parietal as opposed to the frontal electrodes, and very strong to strong evidence against the interaction.

\section{Mean amplitudes for the English targets at frontal and parietal electrodes Condition Related Unrelated}
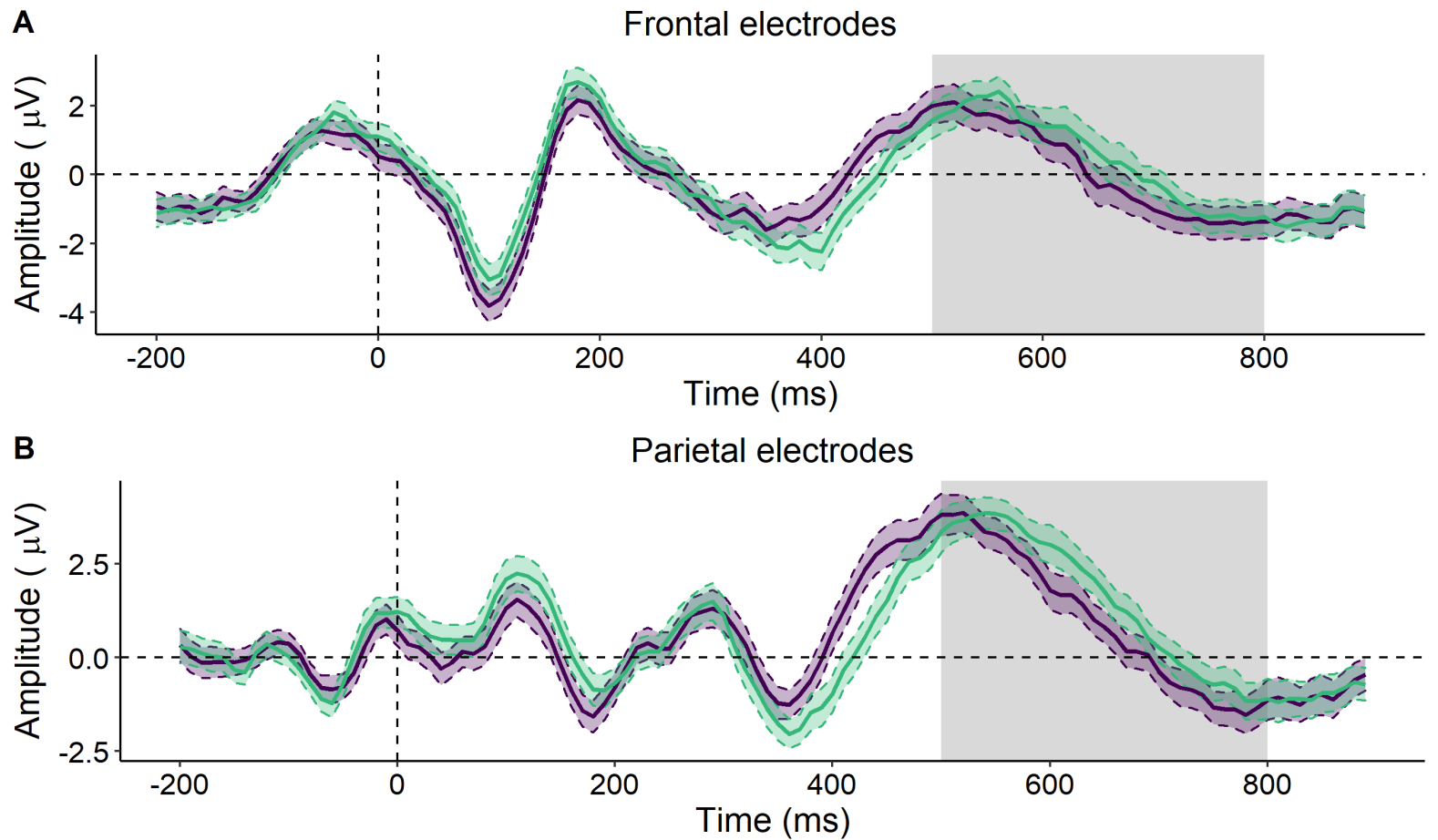

Figure 6. Mean amplitudes (with 95\% confidence intervals) for the semantically related and unrelated English targets. The LPC time window is shaded gray, with panel A showing the amplitudes at frontal electrodes and panel B showing the amplitudes at parietal electrodes. 


\begin{tabular}{lcccr}
\hline Prior & Effect & Estimate $(\mu \mathrm{V})$ & $\mathbf{9 5 \%}$ CrI & $\boldsymbol{B F}_{\mathbf{1 0}}$ \\
\hline \multirow{2}{*}{ Diffuse } & Condition & 0.23 & {$[0.05,0.40]$} & 0.13 \\
& ROI & 0.46 & {$[0.31,0.62]$} & 16611.96 \\
& Condition $\times$ ROI & 0.06 & {$[-0.05,0.16]$} & 0.01 \\
\hline \multirow{2}{*}{ Weakly } & Condition & 0.23 & {$[0.05,0.40]$} & 0.30 \\
informative & ROI & 0.46 & {$[0.31,0.62]$} & 38154.04 \\
& Condition $\times$ ROI & 0.06 & {$[-0.05,0.16]$} & 0.01 \\
\hline \multirow{2}{*}{ Strongly } & Condition & 0.22 & {$[0.05,0.40]$} & 0.76 \\
informative & ROI & 0.46 & {$[0.31,0.62]$} & 72199.27 \\
& Condition $\times$ ROI & 0.06 & {$[-0.05,0.16]$} & 0.05 \\
\hline
\end{tabular}

Table 8. Sensitivity analysis, showing the estimates and the $95 \%$ credible intervals for the LPC priming effects with the English targets as well as the Bayes factors for the analyses with different priors.

\section{Mass univariate analysis}

The amplitudes for semantically unrelated and related English targets differed between about $394 \mathrm{~ms}$ and $465 \mathrm{~ms}$ post onset across a set of bilateral central and parietal electrodes, with more positive values for the related condition than for the unrelated condition. Figure 7 displays significant t-values, topographic maps and mean amplitudes in the two conditions at the electrode $\mathrm{C} 6$, where the t-value was maximal. The analysis also suggested more positive amplitudes in the unrelated than in the related condition at a few parietal electrodes between about $611 \mathrm{~ms}$ and $620 \mathrm{~ms}$ and between $646 \mathrm{~ms}$ and $650 \mathrm{~ms}$ post onset, however, because this difference was only present for a few milliseconds, we do not discuss this further. 
A
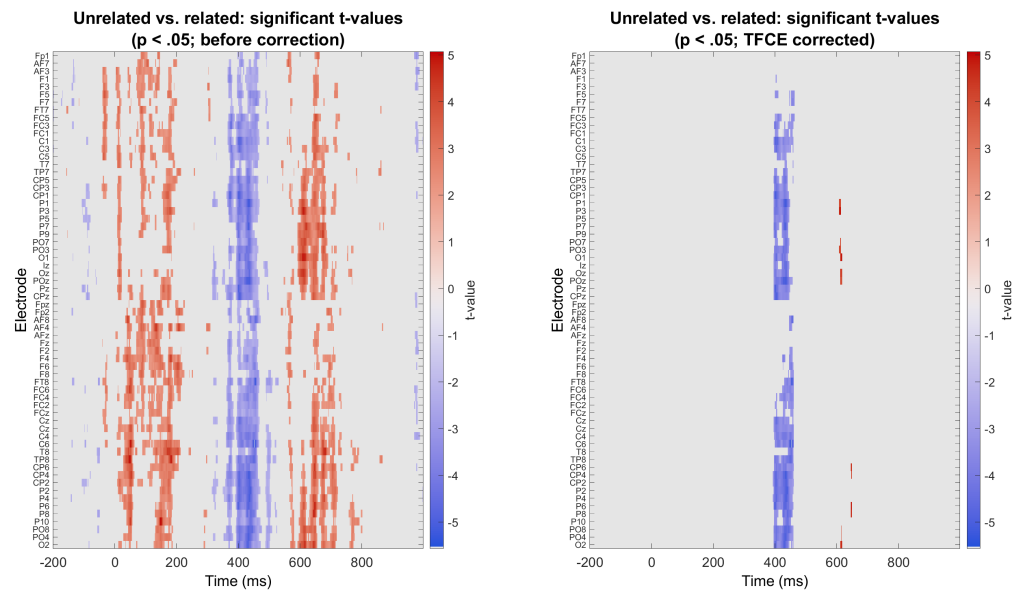

B
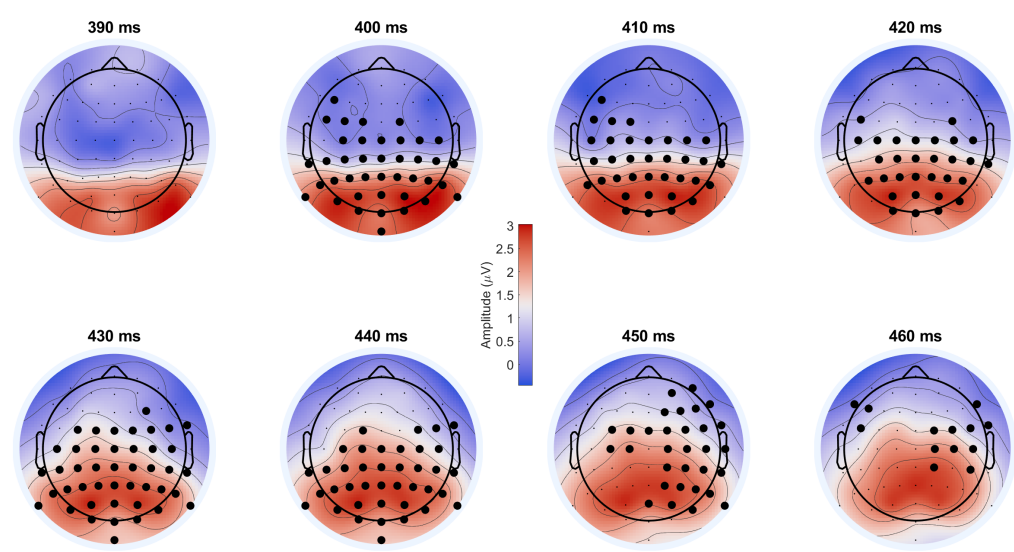

C

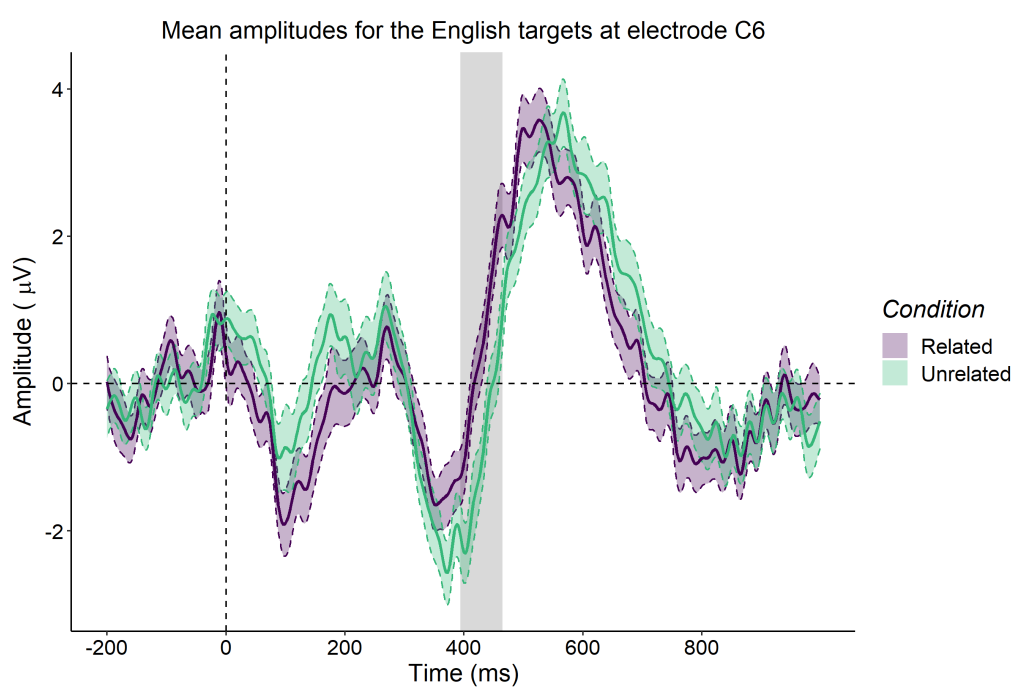

Figure 7. Results of the mass univariate analysis for the comparison between the semantically related and unrelated English targets, with significant t-values before and after TFCE correction (panel A), topographic maps of amplitudes between $390 \mathrm{~ms}$ and $460 \mathrm{~ms}$ post onset (panel B; thick black dots correspond to the electrodes with significant t-values after TFCE) and mean amplitudes in both conditions at the electrode C6 (panel $\mathrm{C}$; the time window between $394 \mathrm{~ms}$ and $465 \mathrm{~ms}$ is shaded gray). 


\section{Main analysis: Priming effects (English prime - Novel name target)}

\section{Analysis of mean amplitudes in two pre-defined spatiotemporal windows}

N400 spatiotemporal window. Figure 8 displays averaged amplitudes for the semantically related and unrelated novel name targets trained in the recent and in the remote sets at centro-parietal electrodes. The models included two predictors, condition (unrelated vs. related) and set (recent vs. remote), and their interaction, and the results of the analyses with all three sets of priors are reported in Table 9. The Bayes factors indicated very strong to strong evidence against a main effect of condition, very strong to moderate evidence against a main effect of set as well as very strong to strong evidence against the interaction.

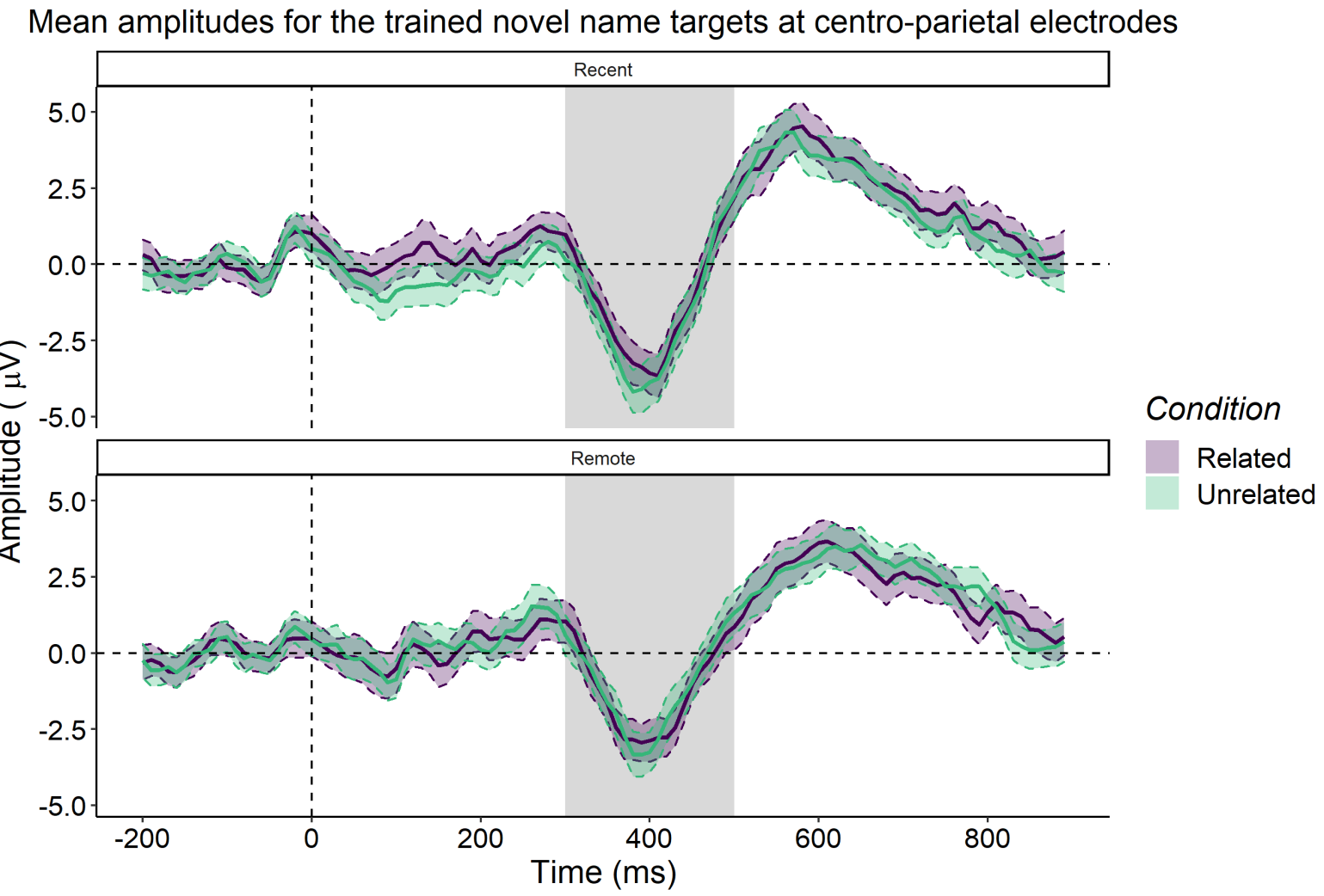

Figure 8. Mean amplitudes (with 95\% confidence intervals) for the semantically related and unrelated novel name targets trained in the recent vs. in the remote set at centroparietal electrodes. The N400 time window is shaded gray.

LPC spatiotemporal window. Figures 9 and 10 display averaged amplitudes for the semantically related and unrelated novel name targets trained in the recent and in the remote sets at the frontal and parietal electrodes. As it was the case for the N400 spatiotemporal window, the fixed effects in all models included condition and set as well as their interaction. 


\begin{tabular}{lcccr}
\hline Prior & Effect & Estimate $(\mu \mathrm{V})$ & $\mathbf{9 5 \%}$ CrI & $\boldsymbol{B F}_{\mathbf{1 0}}$ \\
\hline \multirow{2}{*}{ Diffuse } & Condition & -0.07 & {$[-0.27,0.14]$} & 0.02 \\
& Set & -0.14 & {$[-0.33,0.06]$} & 0.03 \\
& Condition $\times$ Set & -0.08 & {$[-0.27,0.11]$} & 0.01 \\
\hline \multirow{2}{*}{ Weakly } & Condition & -0.06 & {$[-0.26,0.14]$} & 0.02 \\
informative & Set & -0.14 & {$[-0.33,0.06]$} & 0.04 \\
& Condition $\times$ Set & -0.08 & {$[-0.27,0.11]$} & 0.03 \\
\hline \multirow{2}{*}{ Strongly } & Condition & -0.06 & {$[-0.26,0.14]$} & 0.05 \\
informative & Set & -0.14 & {$[-0.34,0.06]$} & 0.12 \\
& Condition $\times$ Set & -0.08 & {$[-0.27,0.11]$} & 0.05 \\
\hline
\end{tabular}

Table 9. Sensitivity analysis, showing the estimates and the $95 \%$ credible intervals for the N400 priming effects with the trained novel name targets as well as the Bayes factors for the analyses with different priors.

The results of the analyses with all three sets of priors are reported in Table 10. For the frontal electrodes, the Bayes factors indicated extreme to very strong evidence against a main effect of condition and set as well as extreme to strong evidence against the interaction. For the interaction. 
Mean amplitudes for the trained novel name targets at frontal electrodes

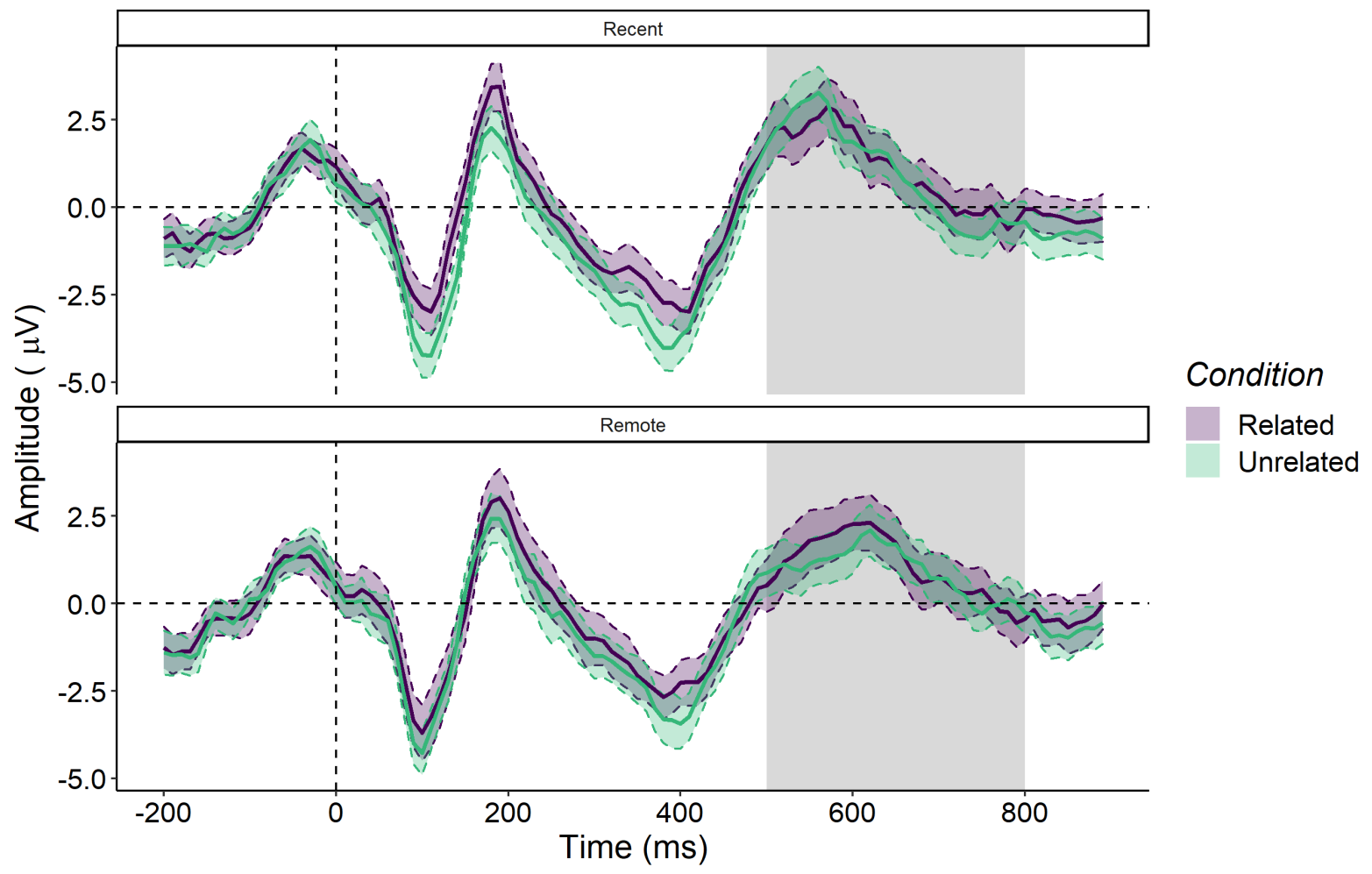

Figure 9. Mean amplitudes (with confidence 95\% intervals) for the semantically related and unrelated novel name targets trained in the recent vs. in the remote set at frontal electrodes. The LPC time window is shaded gray. 
Mean amplitudes for the trained novel name targets at parietal electrodes

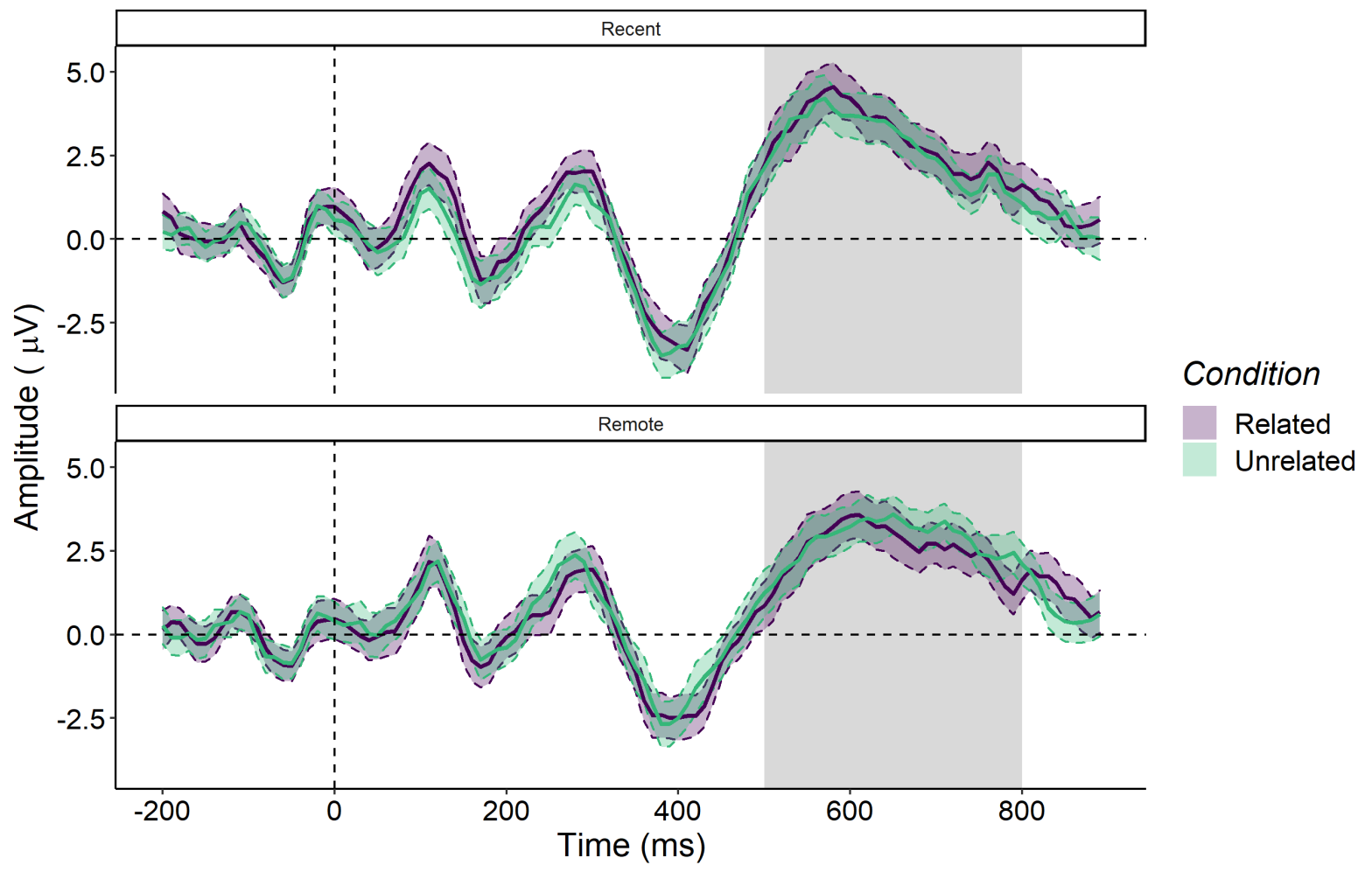

Figure 10. Mean amplitudes (with $95 \%$ confidence intervals) for the semantically related and unrelated novel name targets trained in the recent vs. in the remote set at parietal electrodes. The LPC time window is shaded gray. 


\begin{tabular}{|c|c|c|c|c|c|}
\hline & Prior & Effect & Estimate $(\mu \mathrm{V})$ & 95\% CrI & $B F_{10}$ \\
\hline \multirow{9}{*}{$\begin{array}{l}\text { Frontal } \\
\text { electrodes }\end{array}$} & \multirow{3}{*}{ Diffuse } & Condition & -0.07 & {$[-0.24,0.10]$} & 0.01 \\
\hline & & Set & 0.04 & {$[-0.12,0.21]$} & 0.01 \\
\hline & & Condition $\times$ Set & 0.04 & {$[-0.14,0.23]$} & 0.01 \\
\hline & \multirow{3}{*}{$\begin{array}{l}\text { Weakly } \\
\text { informative }\end{array}$} & Condition & -0.07 & {$[-0.24,0.10]$} & 0.03 \\
\hline & & Set & 0.04 & {$[-0.13,0.22]$} & 0.02 \\
\hline & & Condition $\times$ Set & 0.04 & {$[-0.14,0.22]$} & 0.02 \\
\hline & \multirow{3}{*}{$\begin{array}{l}\text { Strongly } \\
\text { informative }\end{array}$} & Condition & -0.07 & {$[-0.24,0.10]$} & 0.02 \\
\hline & & Set & 0.04 & {$[-0.13,0.21]$} & 0.03 \\
\hline & & Condition $\times$ Set & 0.05 & {$[-0.14,0.23]$} & 0.04 \\
\hline \multirow{9}{*}{$\begin{array}{l}\text { Parietal } \\
\text { electrodes }\end{array}$} & \multirow{3}{*}{ Diffuse } & Condition & 0.01 & {$[-0.15,0.17]$} & 0.01 \\
\hline & & Set & 0.15 & {$[-0.03,0.32]$} & 0.03 \\
\hline & & Condition $\times$ Set & -0.13 & {$[-0.30,0.05]$} & 0.03 \\
\hline & \multirow{3}{*}{$\begin{array}{l}\text { Weakly } \\
\text { informative }\end{array}$} & Condition & 0.01 & {$[-0.15,0.17]$} & 0.01 \\
\hline & & Set & 0.15 & {$[-0.02,0.32]$} & 0.07 \\
\hline & & Condition $\times$ Set & -0.13 & {$[-0.30,0.05]$} & 0.04 \\
\hline & \multirow{3}{*}{$\begin{array}{l}\text { Strongly } \\
\text { informative }\end{array}$} & Condition & 0.01 & {$[-0.15,0.17]$} & 0.05 \\
\hline & & Set & 0.15 & {$[-0.02,0.32]$} & 0.14 \\
\hline & & Condition $\times$ Set & -0.13 & {$[-0.30,0.05]$} & 0.11 \\
\hline
\end{tabular}

Table 10. Sensitivity analysis, showing the estimates and the $95 \%$ credible intervals for the LPC priming effects with the trained novel name targets as well as the Bayes factors for the analyses with different priors. 


\section{Mass univariate analysis}

The recent novel names elicited more positive amplitudes than the remote novel names (main effect of set) between about $506 \mathrm{~ms}$ and $588 \mathrm{~ms}$ across a set of frontal, central and parietal electrodes, and more negative amplitudes than the remote novel names between about $700 \mathrm{~ms}$ and $735 \mathrm{~ms}$ across a set of parietal as well as at some frontal and central electrodes. Figure 11 shows significant t-values, topographic maps and the mean amplitudes for the two sets at the electrode $\mathrm{CP} 4$, where significant t-values were found in both time windows. There was no main effect of condition (unrelated vs. related novel name targets) nor was there an interaction between set and condition as can be seen in Figure 12, which shows that, for both effects, no significant t-values remained after TFCE correction. 
A
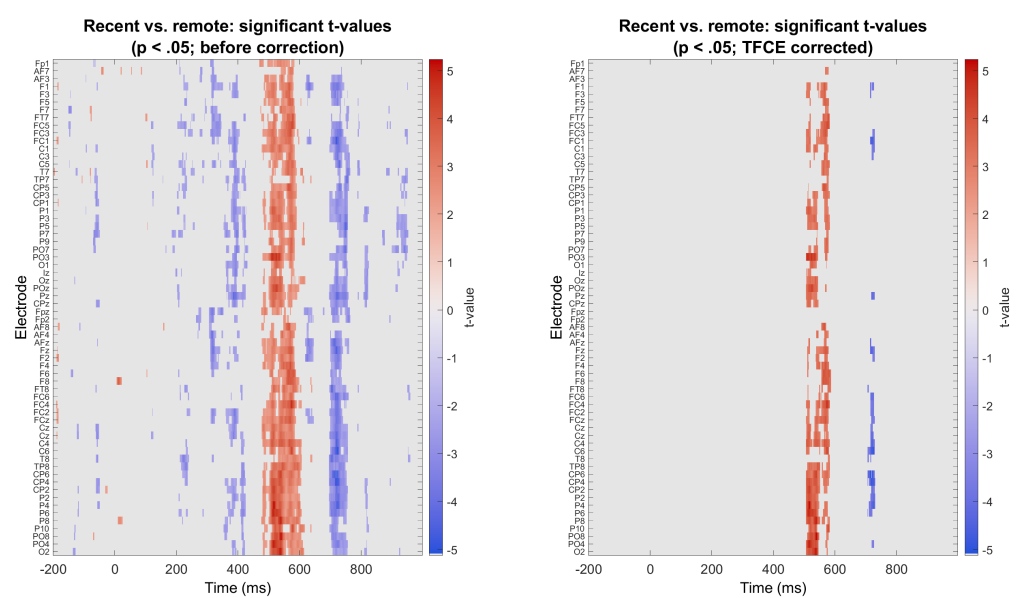

B
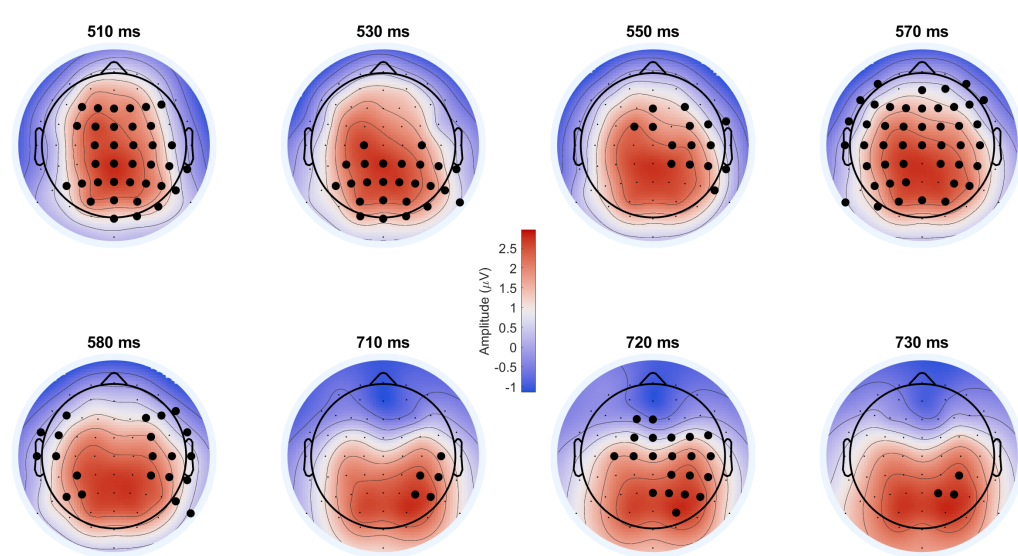

C

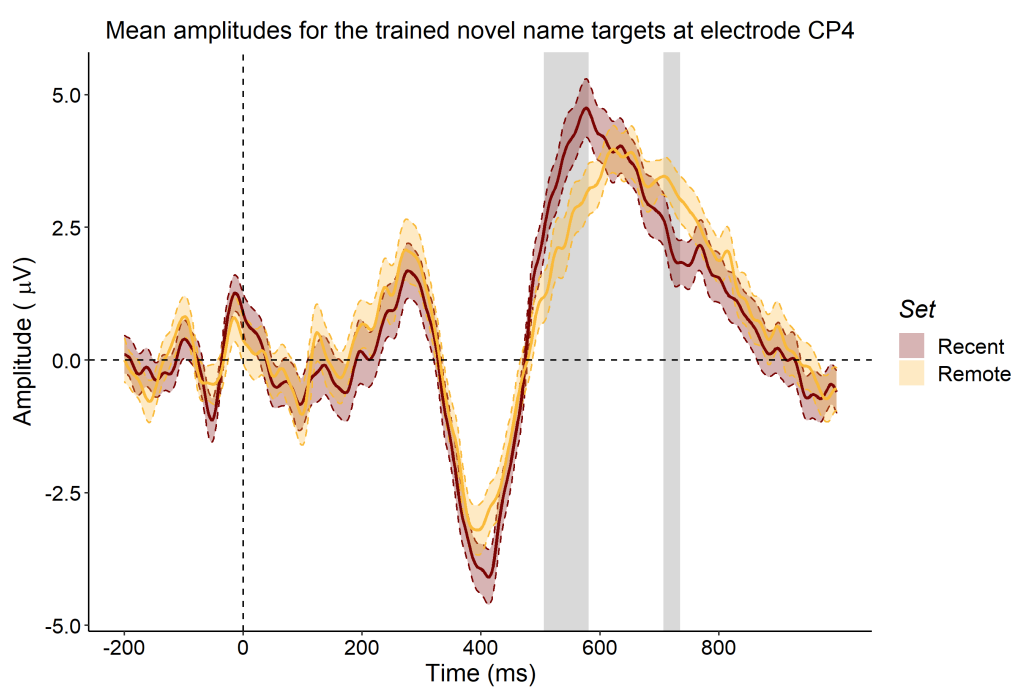

Figure 11. Results of the mass univariate analysis for the comparison between the recent and the remote novel name targets, with significant t-values before and after TFCE correction (panel A), topographic maps of amplitudes between $510 \mathrm{~ms}$ and $580 \mathrm{~ms}$ and between $710 \mathrm{~ms}$ and $730 \mathrm{~ms}$ post onset (panel B; thick black dots correspond to the electrodes with significant t-values after TFCE), and mean amplitudes in both sets at the electrode CP4 (panel C; the time windows between $506 \mathrm{~ms}$ and $581 \mathrm{~ms}$, and between $707 \mathrm{~ms}$ and $735 \mathrm{~ms}$, where the t-values were significant at this particular electrode, are shaded gray). 
A
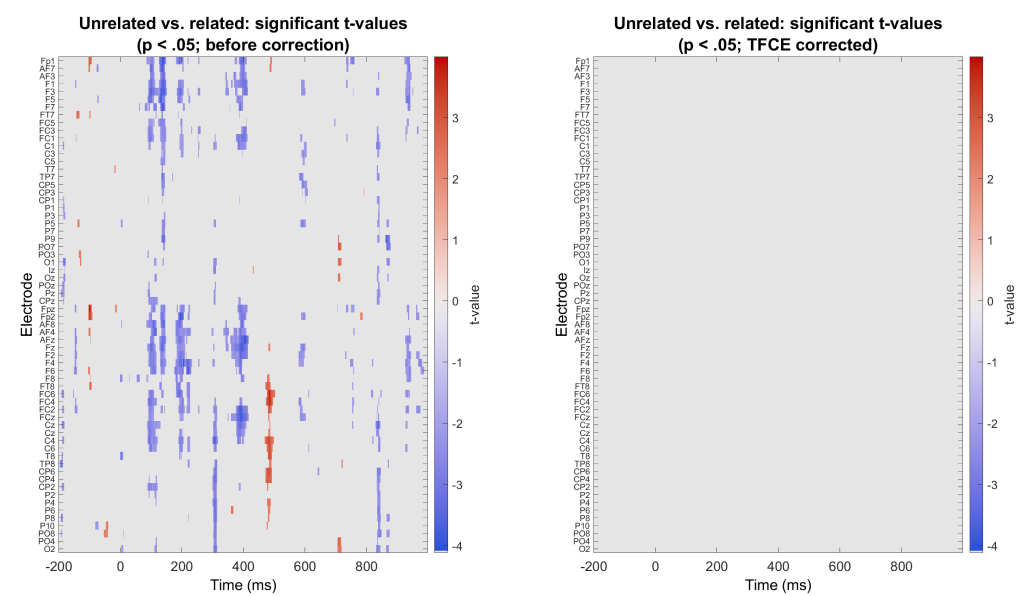

B
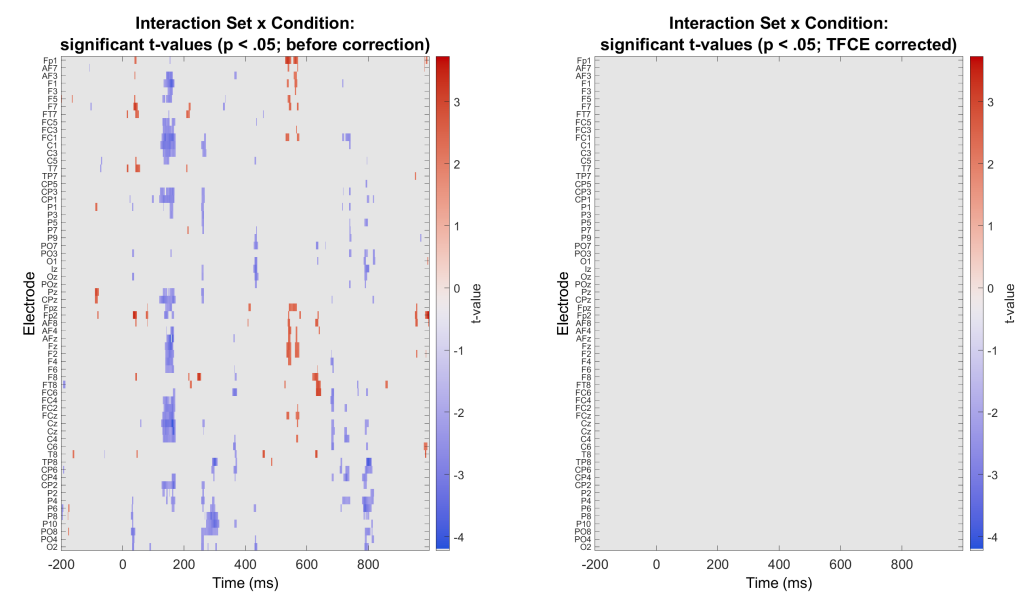

Figure 12. Results of the mass univariate analysis for the comparison between the semantically unrelated and related trained novel name targets (panel A) and the interaction between set and condition (panel B). In both panels, the graph on the left shows significant t-values before correction, and the graph on the right shows significant t-values after correction with TFCE. 


\section{Main analysis: Lexicality effects}

\section{Analysis of mean amplitudes in the pre-defined spatiotemporal window (N400)}

\begin{tabular}{lcccr}
\hline Prior & Contrast & $\begin{array}{c}\text { Estimate } \\
(\mu \mathrm{V})\end{array}$ & $\mathbf{9 5 \%}$ CrI & $\boldsymbol{B F}_{\mathbf{1 0}}$ \\
\hline \multirow{3}{*}{ Diffuse } & Nonwords vs. English words & -0.40 & {$[-0.84,0.04]$} & 3.75 \\
& Recent names vs. nonwords & -0.94 & {$[-1.43,-0.46]$} & 423.43 \\
& Remote vs. recent names & 0.39 & {$[-0.13,0.91]$} & 0.04 \\
\hline \multirow{2}{*}{ Weakly } & Nonwords vs. English words & -0.40 & {$[-0.84,0.03]$} & 6.87 \\
informative & Recent names vs. nonwords & -0.93 & {$[-1.42,-0.45]$} & 846.21 \\
& Remote vs. recent names & 0.39 & {$[-0.12,0.90]$} & 0.09 \\
\hline \multirow{2}{*}{ Strongly } & Nonwords vs. English words & -0.42 & {$[-0.86,0.02]$} & 7.86 \\
informative & Recent names vs. nonwords & -0.93 & {$[-1.40,-0.45]$} & 1046.45 \\
& Remote vs. recent names & 0.38 & {$[-0.13,0.89]$} & 0.10 \\
\hline
\end{tabular}

Table 11. Sensitivity analysis, showing the estimates and the $95 \%$ credible intervals for the lexicality effects in the N400 spatiotemporal window as well as the Bayes factors for analyses with different priors. 


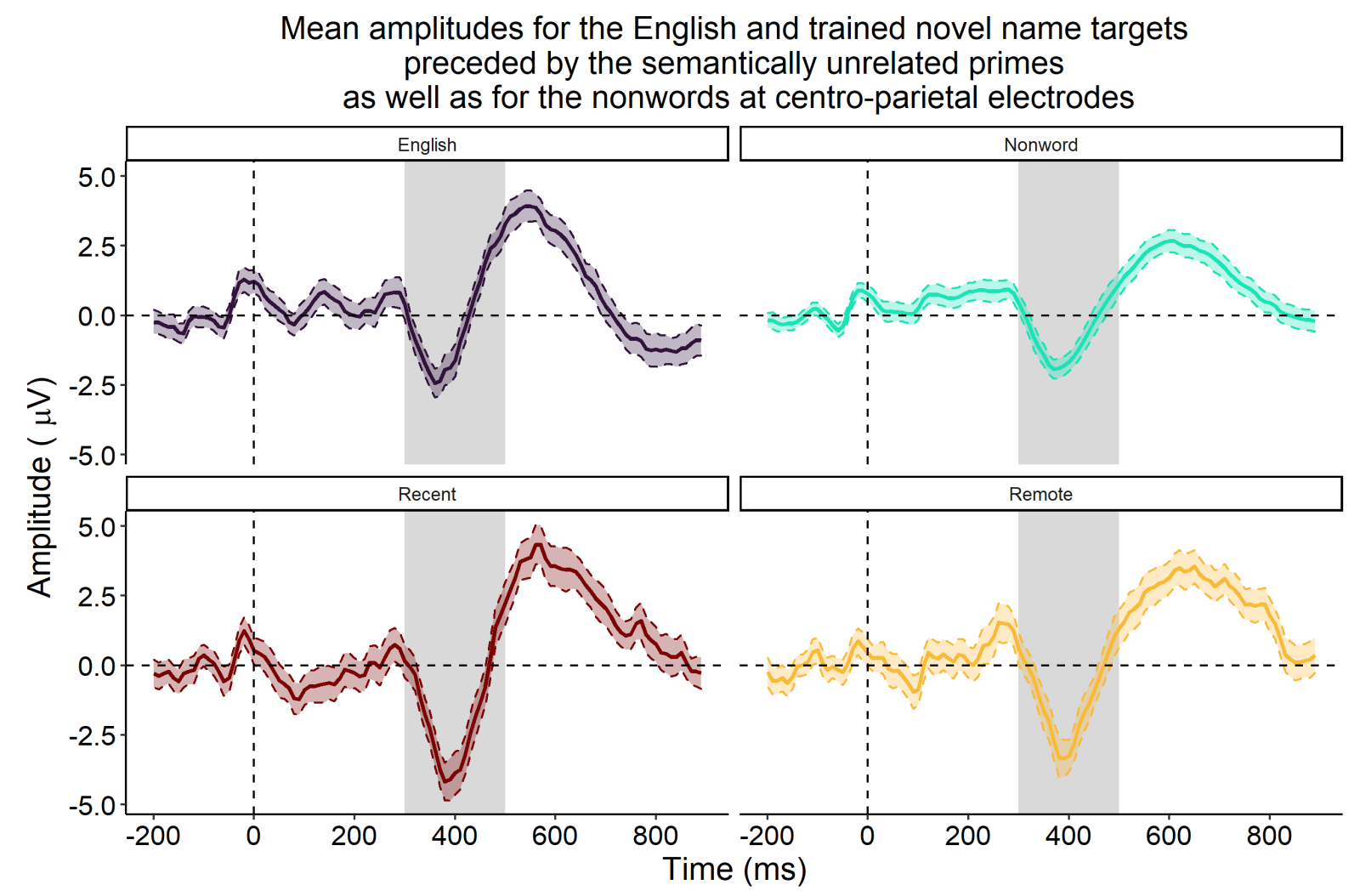

Figure 13. Mean amplitudes (with 95\% confidence intervals) for the nonwords and at the semantically unrelated English and trained novel name (from both sets) targets at centro-parietal electrodes. The N400 time window is shaded gray.

\section{Mass univariate analysis}

\section{Nonwords vs. English targets preceded by semantically unrelated primes.}

The nonwords differed from the English words between about $425 \mathrm{~ms}$ and $598 \mathrm{~ms}$ and then again between $650 \mathrm{~ms}$ and $904 \mathrm{~ms}$ post onset, with the differences first appearing across a small set of central and parietal electrodes, then extending over the whole scalp and, finally, subsiding to a few central and parietal electrodes. Between $425 \mathrm{~ms}$ and $598 \mathrm{~ms}$ post onset, the amplitudes were more negative for the nonwords than for the English words, and, between $650 \mathrm{~ms}$ and $904 \mathrm{~ms}$, more positive for the nonwords than for the English words. Figure 14 illustrates significant t-values, topographic maps and mean amplitudes at the electrode $\mathrm{C} 5$, where the $\mathrm{t}$-value was maximal.

\section{Recent novel name targets preceded by semantically unrelated primes vs.}

nonwords. The novel name targets trained in the recent set differed from the nonwords between about $74 \mathrm{~ms}$ and $286 \mathrm{~ms}$ post onset across a set of frontal and central electrodes, between 
$364 \mathrm{~ms}$ and $464 \mathrm{~ms}$ and between $486 \mathrm{~ms}$ and $698 \mathrm{~ms}$ post onset across sets of frontal, central and parietal electrodes, and between $711 \mathrm{~ms}$ and $759 \mathrm{~ms}$ post onset across a set of frontal electrodes. The recent novel names elicited more negative amplitudes than the nonwords in all the abovementioned time windows except for the time window between $486 \mathrm{~ms}$ and $698 \mathrm{~ms}$, in which the values for the recent novel names were more positive than for the nonwords. Figure 15 shows significant t-values, topographic maps and mean amplitudes at the electrode FC1, which showed significant t-values in all four time windows.

\section{Remote vs. recent novel name targets preceded by semantically unrelated primes. The remote and recent novel names differed between about $538 \mathrm{~ms}$ and $577 \mathrm{~ms}$ post onset across a set of frontal and central electrodes, with more negative amplitudes for the remote than the recent novel names, and between $707 \mathrm{~ms}$ and $758 \mathrm{~ms}$ post onset across a set of frontal, central and parietal electrodes, with more positive amplitudes for the remote than the recent novel names. Significant t-values, topographic maps and mean amplitudes at the electrode Fz, where the t-value was maximal, can be seen in Figure 16.}


A
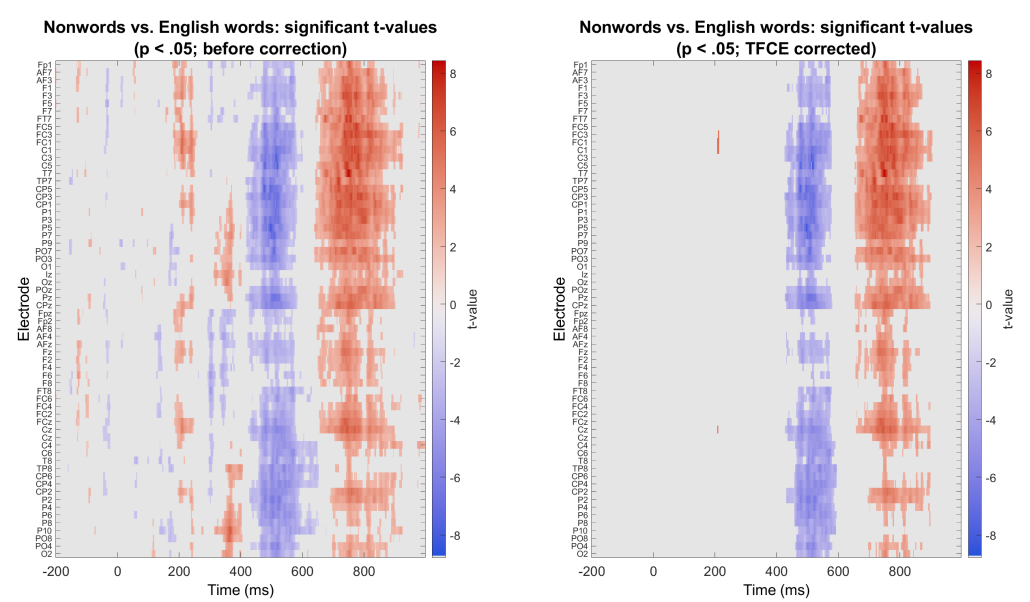

B
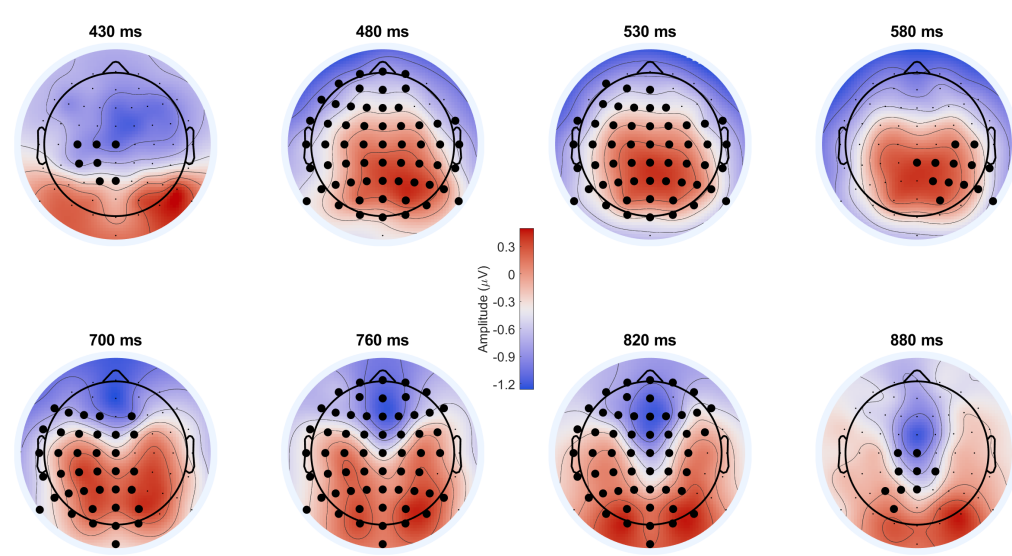

C

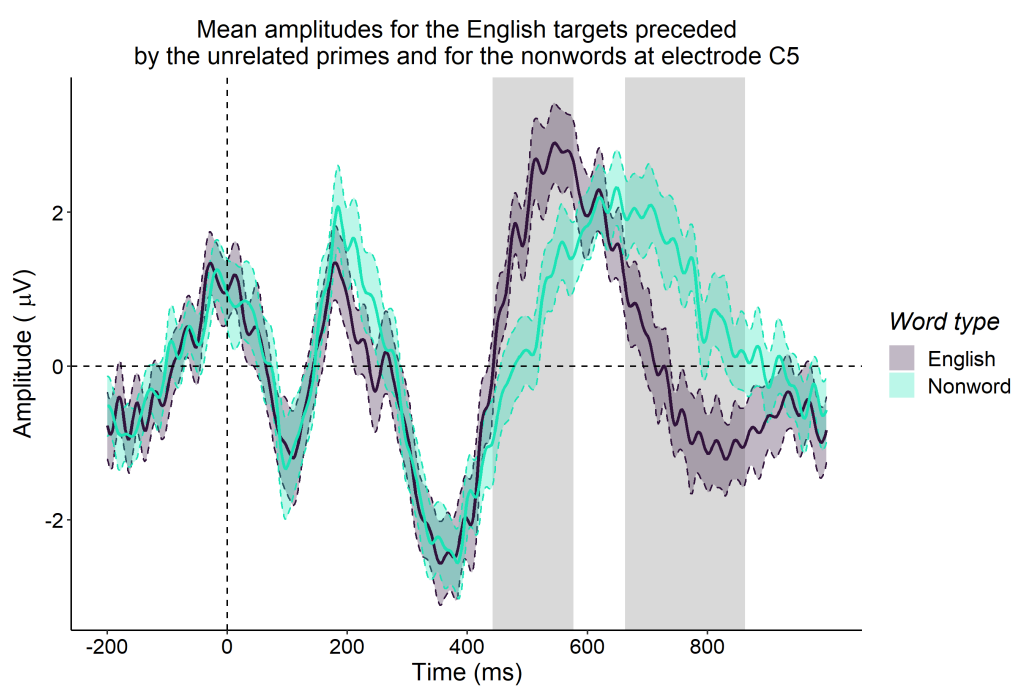

Figure 14. Results of the mass univariate analysis for the comparison between the nonwords and the English targets preceded by semantically unrelated primes, with significant t-values before and after TFCE correction (panel A), topographic maps of amplitudes between $430 \mathrm{~ms}$ and $580 \mathrm{~ms}$ and between $700 \mathrm{~ms}$ and $880 \mathrm{~ms}$ post onset (panel B; thick black dots correspond to the electrodes with significant t-values after TFCE), and mean amplitudes at the electrode C5 (panel C; time windows $442-577 \mathrm{~ms}$ and $663-863 \mathrm{~ms}$, where the t-values were significant at this particular electrode, are shaded gray). 
A
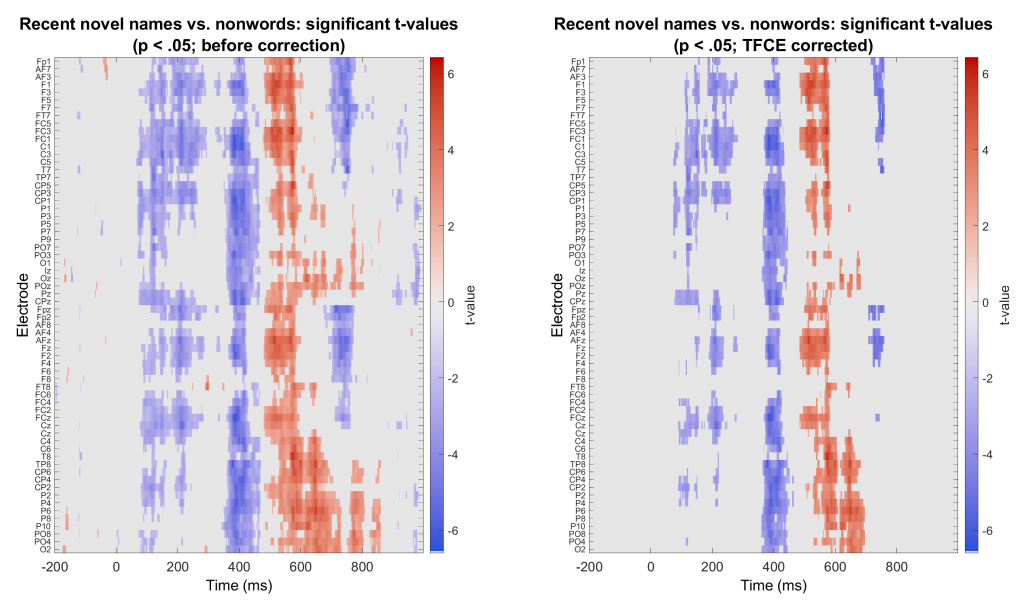

B
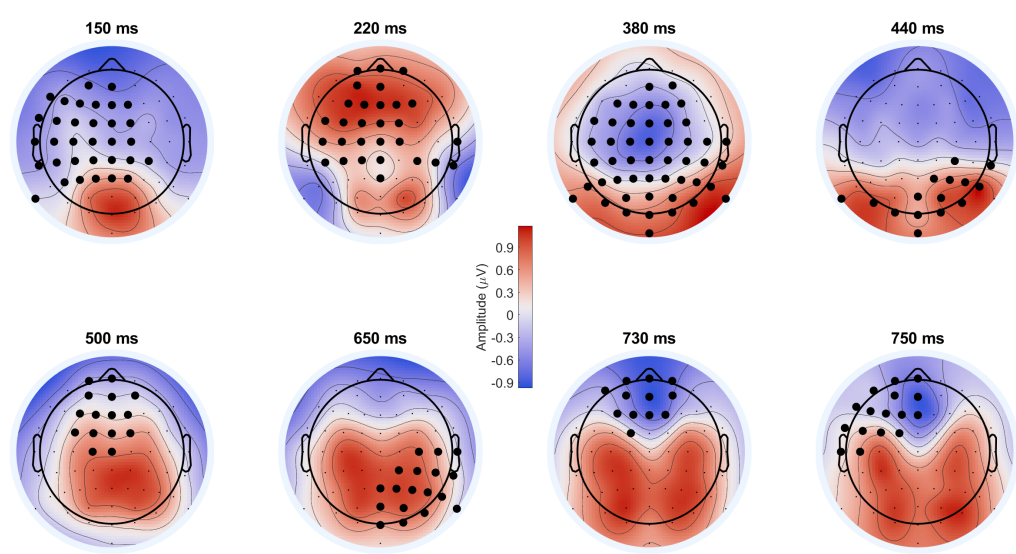

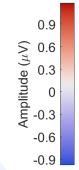
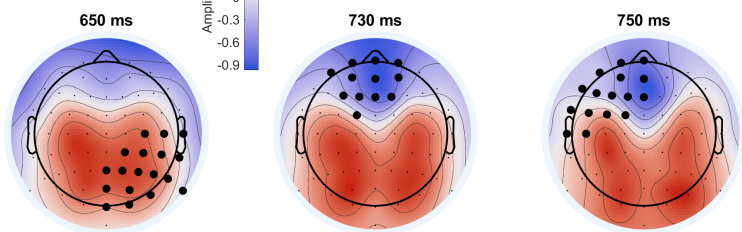

C

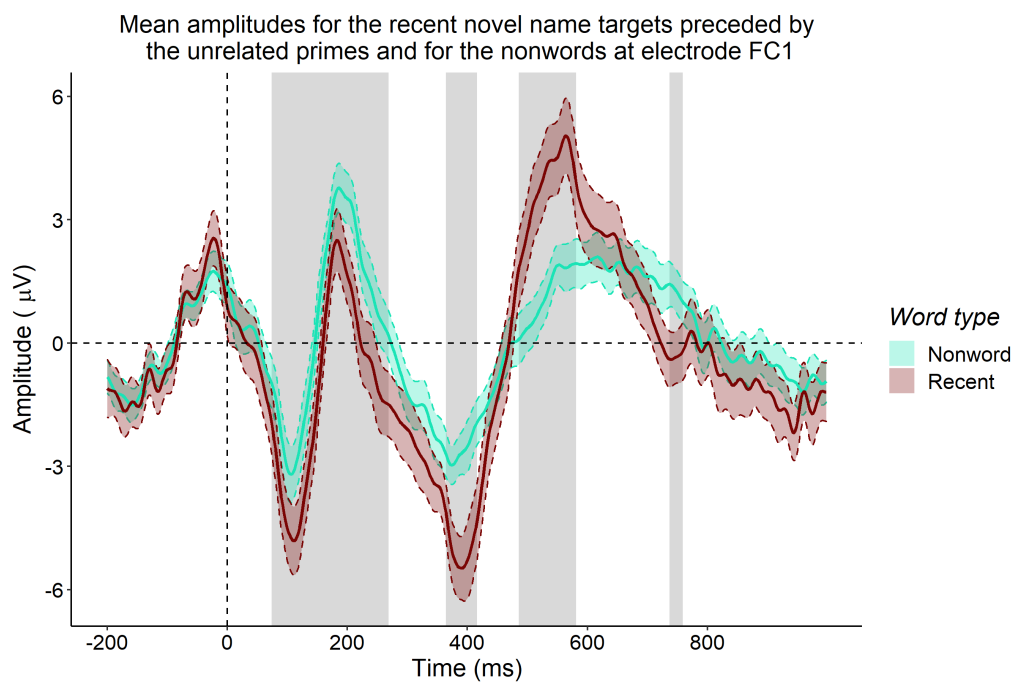

Figure 15. Results of the mass univariate analysis for the comparison between the recent novel name targets preceded by semantically unrelated primes and the nonwords, with significant t-values before and after TFCE correction (panel A), topographic maps of amplitudes between $150 \mathrm{~ms}$ and $750 \mathrm{~ms}$ post onset (panel B; thick black dots correspond to the electrodes with significant t-values after TFCE) and mean amplitudes at the electrode FC1 (panel C; time windows $74-269 \mathrm{~ms}, 364-416 \mathrm{~ms}, 486-581 \mathrm{~ms}$ and $737-759 \mathrm{~ms}$, where the t-values were significant at this particular electrode, are shaded gray). 
A
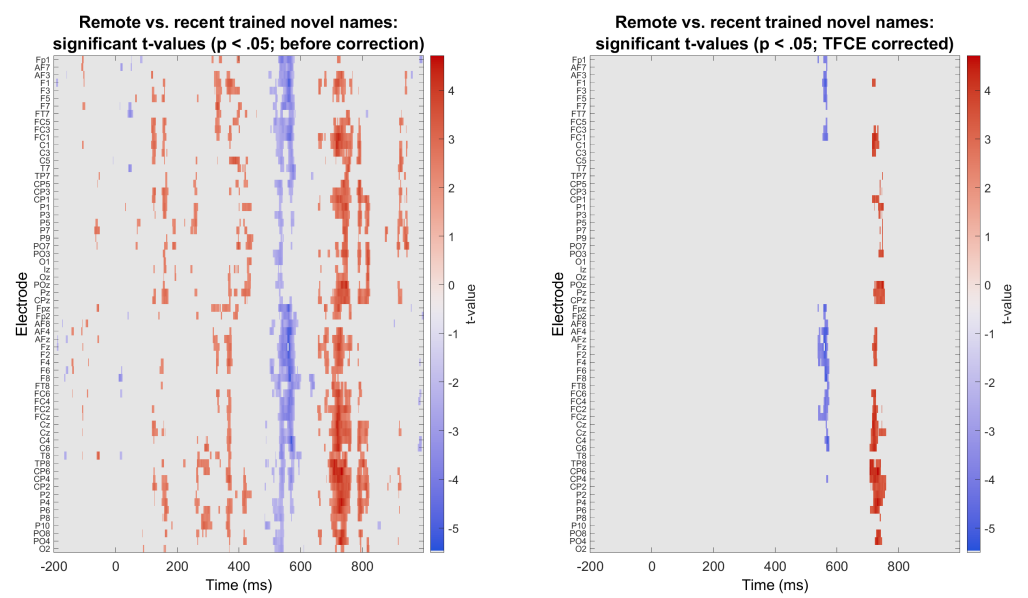

B
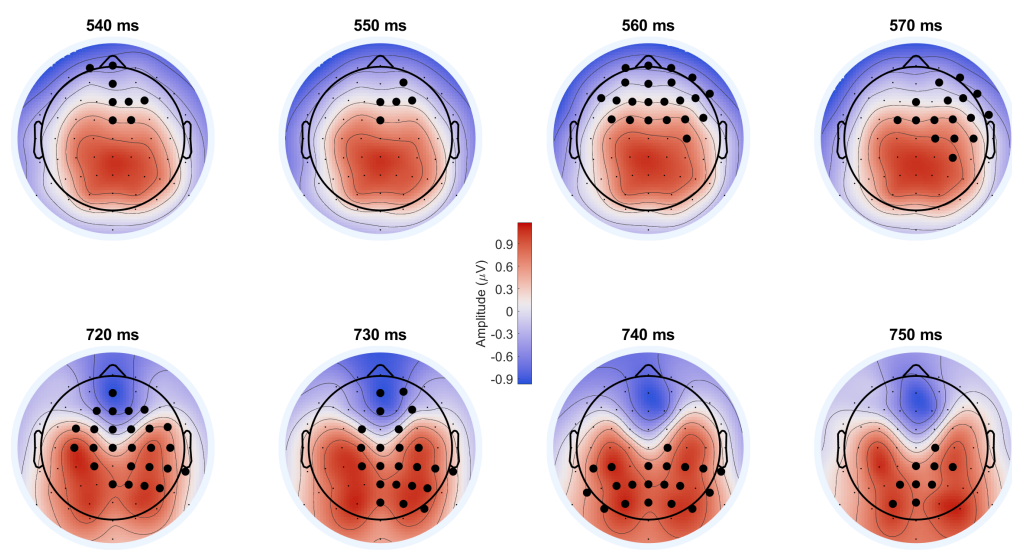

C

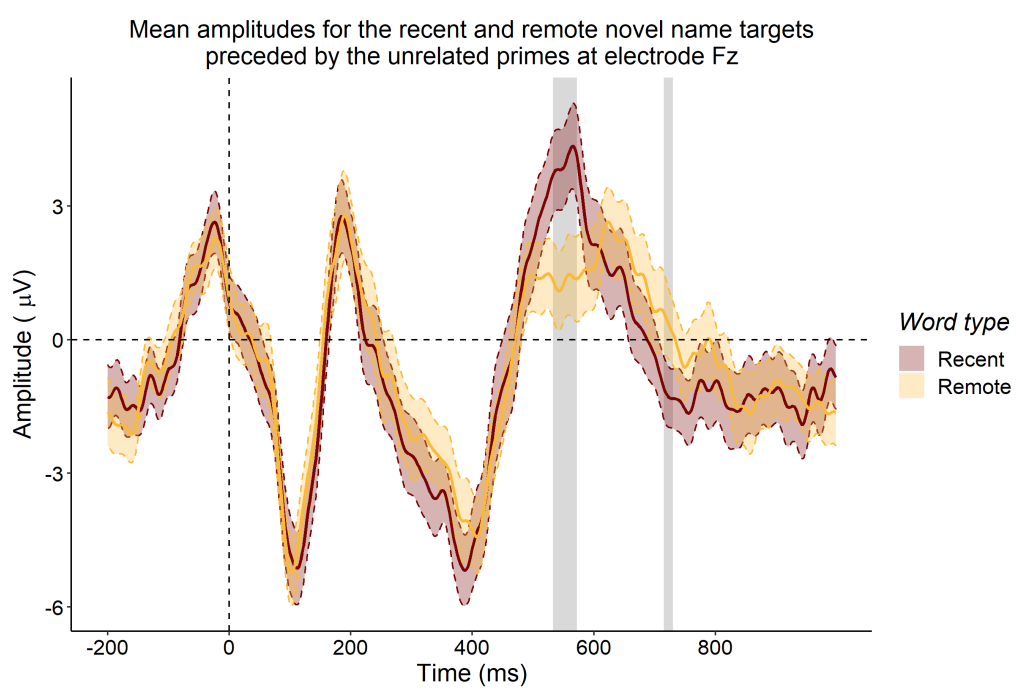

Figure 16. Results of the mass univariate analysis for the comparison between the remote and recent novel name targets preceded by semantically unrelated primes, with significant t-values before and after TFCE correction (panel A), topographic maps of amplitudes between $540 \mathrm{~ms}$ and $750 \mathrm{~ms}$ post onset (panel B; thick black dots correspond to the electrodes with significant t-values after TFCE) and mean amplitudes at the electrode $\mathrm{Fz}$ (panel C; time windows 533-572 ms and $715-730 \mathrm{~ms}$, where the t-values were significant at this particular electrode, are shaded gray). 


\section{Discussion}

The present study investigated how behavioural markers of integration map onto electrophysiological markers of integration in learning of novel names for novel concepts. The participants learned two sets of novel names, one set on each of two consecutive days (remote and recent sets, respectively), and performed a continuous primed lexical decision task immediately after learning of the recent set. In the priming task, familiar English words were primes, and (semantically related and unrelated) familiar English (preliminary analysis) and trained novel names (main analysis) were targets.

To our knowledge, this is the first study to use the continuous primed lexical decision task to assess integration, and the first study to examine the N400 and the LPC components in this task (with both familiar and trained novel names). First, we summarise and interpret the results of the semantic priming analysis for the English targets because they provide a baseline against which the novel names can be compared. We then move on to discussing the semantic priming and the lexicality effects for the trained novel names.

\section{Semantic priming effects for the English words}

Our analysis provided evidence in favour of a behavioural semantic priming effect of approximately $20 \mathrm{~ms}$ for the English targets, with longer response times for the targets unrelated to the primes than for those semantically (but not associatively) related to the primes. This finding replicates some of the previous studies (e.g., de Mornay Davies, 1998; McRae \& Boisvert, 1998, but see Shelton \& Martin, 1992, Experiment 4; Moss et al., 1995, Experiment 3) and is in accord with several models of semantic processing including spreading activation (e.g., Collins \& Loftus, 1975; Lerner et al., 2012) and compound cue (Ratcliff \& McKoon, 1988) models as well as the distributed model of semantic memory (McRae et al., 1997), with all three predicting that semantically related words should prime each other even if they are not associatively related.

Our electrophysiological findings were consistent with the behavioural data. The mean amplitude in the N400 spatiotemporal window was more negative when the English targets were preceded by the unrelated primes than by the semantically related primes, and the mass univariate analysis showed that this effect started at about $390 \mathrm{~ms}$ post target onset and lasted up 
to $465 \mathrm{~ms}$ post target onset. While numerous previous studies have reported the N400 semantic priming effect in standard (paired) primed lexical decision and semantic-relatedness judgement tasks, among others (see, e.g., Kutas \& Federmeier, 2011, for a review), to our knowledge, the current study is the first to demonstrate this effect in a continuous primed lexical decision task. It is intriguing that the transition from negativity to positivity in the unrelated condition seemed to lag slightly behind that in the related condition (see Figures 5 and 7 ). In line with the distributed model of semantic memory, one explanation of this phenomenon could be that the speed with which the target word meaning is computed is influenced by the amount of featural overlap between the prime and the target, leading to slower processing in the unrelated condition.

Finally, our analysis also yielded evidence against a difference between the related and the unrelated conditions in the LPC spatiotemporal window. We note that evidence in favour of the null hypothesis can have many causes and whether it signals a true absence of effect warrants further investigation. Assuming that it does, this result contrasts with some of the previous studies in word recognition literature, where an LPC priming effect was observed both in standard primed lexical decision (e.g., Fang \& Perfetti, 2017; Hill et al., 2002) and in primed semantic-relatedness judgement tasks (e.g., Bakker et al., 2015; Liu \& van Hell, 2020). Yet, given the nature of the primed continuous lexical decision task, this discrepancy is hardly surprising. One possible reason is that the LPC component is believed to reflect controlled lexical-semantic processing (e.g., Olichney et al., 2000; Rugg \& Curran, 2007; Van Petten et al., 1991), and, as we have argued in the Introduction, both the standard primed lexical decision and the semanticrelatedness judgement tasks are sensitive to these controlled processes.

In summary, we used a task that is assumed to tap into automatic processes of lexical-semantic retrieval and to diminish strategic and controlled processing. Our findings for the English words are in line with these assumptions and provide information on the neural signatures of automatic semantic processing of familiar words. These findings further suggest that the primed continuous lexical decision task may provide a better measure of those behavioural and electrophysiological markers of integration that rely on lexical competition than the paired primed lexical decision and the semantic-relatedness judgement tasks. With this in mind, we now turn to our findings for the trained novel names. 


\section{Semantic priming effects for the trained novel names}

In contrast to our finding with the English words, we obtained evidence against a difference in response times to the trained novel names primed by related versus those primed by unrelated English words. This was true whether the novel names had been trained in the same session (recent set) or one day earlier (remote set). This suggests that the familiar English words did not facilitate recognition of the trained novel names either immediately or after a 1-day consolidation period.

This result is at odds with some of the previous studies. For instance, van der Ven et al. (2015) reported a behavioural priming effect in a paired primed lexical decision task after a 1day consolidation opportunity, whereas Tamminen and Gaskell (2013) found that priming effects were of similar magnitude ( $8 \mathrm{~ms})$ across different consolidation opportunities (0-day, 1-day, 7day) for both masked and unmasked paired primed lexical decision. Critically, however, the unmasked paired version of the primed lexical decision task does not allow the disentanglement of automatic processes of lexical-semantic retrieval from controlled processes resulting from the task demands (e.g., Neely, 1991). Moreover, while Tamminen and Gaskell's original results are intriguing, the authors, suspecting that the lack of differences between the three consolidation periods was due to low power, conducted an additional analysis combining the data from both versions of the lexical decision task. This analysis only tested the contrast between the 7-day and the 0-day consolidation opportunities and, consequently, Tamminen and Gaskell's results remain inconclusive with respect to the comparison examined in our experiment. As a final observation, in contrast to our study, both van der Ven et al. (2015) and Tamminen and Gaskell (2013) used the trained novel names as primes rather than targets (although it remains unclear whether (and why) this could have influenced priming). Therefore, a possible account of our findings is that, when the influence of explicit and strategic processing is minimised, recognition of the trained novel names is not facilitated by familiar words either immediately or 24 hours after exposure.

The electrophysiological findings corroborate this conclusion: whether the novel names had a 0-day or a 1-day consolidation opportunity, we obtained evidence against a difference between the related and unrelated conditions in both the N400 and the LPC spatiotemporal windows. Assuming that this result truly reflects absence of priming effects, this finding gives 
insight into the conflicting results of the previous EEG studies. Using the semantic-relatedness judgement task, Bakker et al. (2015) and Liu and van Hell (2020) found no N400 priming effects either immediately after, or one day after (Bakker et al., 2015; Liu \& van Hell, 2020), or even seven days after (Liu \& van Hell, 2020) exposure, whereas Balass et al. (2010) and Perfetti et al. (2005) observed the N400 priming effect immediately after training. While it is tempting to relate these immediate effects to integration, the semantic-relatedness judgment task is a paradigm that necessarily involves controlled semantic processing. This is noteworthy because the N400 component is known to be sensitive to task demands, with larger priming effects when instructions explicitly call for semantic analysis (e.g., Chwilla et al., 1995), and can therefore index both automatic and controlled processes of lexical retrieval (Kutas \& Federmeier, 2011). Furthermore, in Perfetti et al. (2005), some of the primes had been associated with the targets already during training, which could have further enhanced the contribution of controlled processing to task performance. On these grounds, the presence of the immediate N400 priming effects in Balass et al. (2010) and Perfetti et al. (2005) cannot be unequivocally attributed to automatic processes of lexical-semantic retrieval and, consequently, their insights regarding integration are uncertain.

Features of the two studies that have reported immediate N400 priming effects in the paired primed lexical decision task (Borovsky et al., 2012, 2013) also throw doubts on whether these effects indeed reflect integration. In Borovsky et al. $(2012,2013)$, the priming task was interleaved with training, which required the participants to read pairs of sentences where the novel names always appeared in the sentence-final position. Because Borovsky and collaborators had instructed their participants to try and make sense of the sentences rather than learn the new words themselves, their results might not necessarily reflect learning. Their design thus makes it feasible that the priming effects could be, at least to some extent, driven by integrative discourse processing which entails activation of semantic and syntactic features of words constituting the sentences, retrieval of world knowledge and prediction of the upcoming information, among other processes (e.g., Sparks \& Rapp, 2010). Discourse processing is known to modulate both the N400 and the LPC components (e.g., Brouwer et al., 2012; van Berkum, 2009; van Berkum et al., 1999), while it is also characterised by differential involvement of the brain hemispheres (e.g., Tompkins et al., 2008).

To summarise, we have argued that our behavioural and electrophysiological data suggest 
that neither the 0-day nor the 1-day consolidation period was sufficient in our study for integration of novel names for novel concepts into semantic memory. We have suggested that the discrepancy with other studies is most likely due to the primed continuous lexical decision task being less susceptible to controlled processes of lexical-semantic retrieval than the tasks used previously. Importantly, while the outcome of the analyses discussed so far is indicative of lack of integration for both sets of novel names, the results of our remaining analyses suggest that processing of the novel names in the priming task differed depending on the length of the consolidation opportunity. We now turn to the discussion of these results and their implications.

The first two findings relevant in this regard are the behavioural and electrophysiological effects of set (remote vs. recent). At the behavioural level, we obtained (moderate) evidence that response times for the novel name targets trained in the remote set were about $11 \mathrm{~ms}$ longer than for the novel names trained in the recent set. This difference suggests that discriminating the novel names from nonwords was more difficult for the remote names, possibly due to a longer delay between the time of training and the priming task than for the recent set.

At the electrophysiological level, the mass univariate analysis revealed that the recent novel names showed more positive and more negative amplitudes than the remote novel names between $506-581 \mathrm{~ms}$ and between $707-735 \mathrm{~ms}$, respectively. Given the temporal and the spatial distributions of these effects, it seems reasonable that they might index two distinct processes in recognition memory, familiarity and recollection (see Yonelinas, 2002, for a review). While familiarity is thought to reflect the experience of recognising a stimulus as familiar, recollection has been associated with the ability to retrieve the associative information about an event in which the stimulus was encountered (e.g., when and where a particular word was trained). Most models of memory recognition assume that, at retrieval, recollection and familiarity are initiated in parallel, but do not depend on each other (Yonelinas, 2002), rely on different brain regions (e.g., Diana et al., 2007; Eichenbaum et al., 2007) and have different electrophysiological correlates (e.g., Cansino \& Trejo-Morales, 2008; Rugg \& Curran, 2007; Yu \& Rugg, 2010). Familiarity is believed to occur quicker than recollection and has been linked to modulations of the Frontal N400 (or, FN400) component, whereby familiar items exhibit more positivity than new items at frontal-central sites between $400 \mathrm{~ms}$ and $600 \mathrm{~ms}$ post stimulus onset (note, however, that some studies have argued that the 
FN400 is not a separate ERP component but the N400 elicited when processing is facilitated by prior exposure to the stimulus, see, e.g., Paller et al., 2007; Voss \& Paller, 2007). In turn, better recollection has been associated with an increase in the LPC. Therefore, in our study, the enhanced positivity for the recent novel names between 500-600 ms post onset could be indicative of higher familiarity with those names compared to the remote novel names. Indeed, since the priming task was administered immediately after learning of the recent set but about 24 hours after learning of the remote set, higher familiarity and better recollection seems likely for the recent compared to the remote set. One could further hypothesise that, for the remote novel names, familiarity may have been too low to enable their discrimination from the nonwords, prompting the participants to attempt to remember whether they had seen these novel names during training. These attempts to retrieve episode-specific information, whether successful or not, could have given rise to the more positive amplitudes for the remote novel names between $700-800 \mathrm{~ms}$ post onset observed in our study.

This account is also in line with our finding of evidence against differences between the two sets in the analysis of the mean amplitudes in the LPC spatiotemporal window, which included frontal and parietal electrodes. We included the frontal electrodes following the literature suggesting that frontal brain potentials between $500-800 \mathrm{~ms}$ post stimulus onset are sensitive to the type of source information retrieved from episodic memory (e.g., Peters \& Daum, 2009). However, in our study, the differences between the two sets were found across a set of mostly frontal and central electrodes between about $500 \mathrm{~ms}$ and $600 \mathrm{~ms}$ and across a small set of mostly central and parietal electrodes between about $700 \mathrm{~ms}$ and $735 \mathrm{~ms}$ post onset. While the distribution of the former effect corresponds to the typical distribution of the FN400 component, the distribution of the latter effect is broader than the typical LPC. Following other reports in the literature (e.g., Addante et al., 2012), we hypothesise that this broader negativity was due to low recognition confidence for the remote novel names. Nevertheless, this preliminary explanation requires further investigation in the future.

In sum, above we have argued that the main effect of set found in both the behavioural and the electrophysiological data could be taken to index differential processing of the remote and the recent novel names in episodic memory. More specifically, we have suggested that these differences could be due to higher familiarity and better recollection of the recent as opposed to the remote novel names. In our view, this interpretation can also account for the results of the 
lexicality analysis, which we discuss in the remainder of this section.

\section{Lexicality effects}

The results of the lexicality analysis comparing the recent and remote novel name targets preceded by unrelated primes followed the same pattern as the results of the main effect of set in the semantic priming analysis discussed above. Firstly, the analysis of mean amplitude in the (pre-defined) N400 spatiotemporal window provided evidence against a difference between the two sets. This finding could suggest that, 24 hours after exposure to the remote novel names, their processing does not differ from that of the recent novel names. Secondly, the mass univariate analysis showed that, later in the time course, the novel names from the two sets did differ from each other. Specifically, more positive amplitudes were observed for the recent than the remote novel names across a set of frontal and central electrodes between about $530 \mathrm{~ms}$ and $580 \mathrm{~ms}$ post onset. In addition, between about $700 \mathrm{~ms}$ and $760 \mathrm{~ms}$ post onset, the amplitudes were more negative for the recent novel names across a set of central and parietal as well as across a few frontal electrodes. As noted above, a possible explanation for these effects could be that lower familiarity of the remote novel names necessitated a conscious recollection process in order to discriminate them from the previously unseen nonwords.

Moreover, the analysis of mean amplitude in the N400 spatiotemporal window also revealed strong evidence for more negative amplitudes for the recent novel names (that followed unrelated primes) as compared to the previously unseen nonwords. The mass univariate analysis indicated that this effect was also present across a set of frontal electrodes and that it lasted from about $360 \mathrm{~ms}$ to $460 \mathrm{~ms}$ post onset. In turn, the nonwords elicited more negativity than the English words (preceded by the unrelated primes) between about $425 \mathrm{~ms}$ and $600 \mathrm{~ms}$ post onset, with this effect first appearing across a few centro-parietal electrodes and later extending over the whole scalp (note that, because this effect emerged close to the end of the N400 spatiotemporal window (300 -500 ms post onset), the evidence in favour of this effect in the analysis on mean amplitude in the pre-defined spatiotemporal windows was only moderate).

Taken together, these findings offer an interesting insight in the mechanisms of word learning. In the word recognition literature, more negative N400 responses have been observed to previously unseen nonwords than familiar words in unprimed lexical decision (e.g., Bentin, 1987; Bentin et al., 1985; Holcomb et al., 2002). This phenomenon is usually explained as 
follows: when a stimulus, either a word or a nonword, is encountered, it is believed to activate its lexical neighbours and their semantic representations (Grainger \& Jacobs, 1996; Holcomb et al., 2002). As processing continues, if the stimulus is a word, lexical activity associated with it suppresses the activity of its lexical neighbours. However, if the stimulus is a nonword, no suppression can occur, leading to larger (more negative) N400 responses. Following this logic, one would expect trained novel names to exhibit less negative responses than nonwords unless the novel names are treated as nonwords, in which case an N400 response of similar magnitude would be expected. In our study, however, the recent novel names showed more negativity than the previously unseen nonwords. One possible account is that, due to recent exposure, the representations of the recent novel names were still very active in episodic memory, giving rise to probabilistic expectations about the presence of representations in semantic memory. However, because no lexical entries would have been available for the novel names, these expectations could not have been met, requiring an update of (linguistic) predictions and, thus, a larger (more negative) N400 response (and see Rabovsky et al., 2018; Rabovsky \& McClelland, 2020, for a model in which the N400 is believed to reflect a change in probabilistic representation of meaning).

Interestingly, in our study, the differences between the recent novel names and the nonwords emerged very early, with greater negativity for the nonwords starting from about $70 \mathrm{~ms}$ post onset (across a set of frontal and central electrodes) and persisting up to almost 300 ms post onset (which was then followed by the N400 lexicality effect). According to models of (psycholinguistic) information processing, electrophysiological effects occurring before $250 \mathrm{~ms}$ post stimulus onset could index initial lexical, semantic and syntactic processes which are crucial for unambiguous identification of language elements (see, e.g., Fodor, 1983; Friederici, 2002, for a cascaded model of information processing, and Pulvermüller et al., 2009, for a parallel model of psycholinguistic information access). The literature on visual word recognition lends support for these assumptions: retrieval of lexical-semantic information has been shown to be underway as early as at around $160 \mathrm{~ms}$ post word onset (Amsel et al., 2013; Hauk et al., 2012), with differences between familiar words and nonwords emerging already during the N100-P200 ERP complex (Rugg, 1983), while posterior negativity at $200 \mathrm{~ms}$ post onset (the so-called N200 component) has been linked with initial orthographic processing (Dehaene et al., 2001; McCandliss et al., 2003). In line with this literature, our finding could 
indicate that the trained novel names were already distinguished from the nonwords early in processing.

The electrophysiological correlates of the nonwords and the recent novel names also differed later in the processing, between about $480 \mathrm{~ms}$ and $700 \mathrm{~ms}$ post onset, with the trained novel names showing more positivity across a large set of frontal, central and parietal electrodes. This effect was followed by enhanced negativity to the trained novel names across a few frontal electrodes between about $710 \mathrm{~ms}$ and $760 \mathrm{~ms}$ post onset. One possible account of these effects is that they reflect differences in familiarity and recollection. Firstly, because the participants had been exposed to the recent novel names only a few minutes prior to the lexical decision task, greater positivity for the novel names in the first time window (before $700 \mathrm{~ms}$ post onset) could reflect both reasonably high recognition confidence as well as the ability to retrieve episodic information related to training. Greater positivity for the nonwords after $700 \mathrm{~ms}$ post onset might then indicate the participants' attempts to consciously recall whether they had seen these stimuli before. This could also explain why the nonwords showed greater positivity than the English words between about $650 \mathrm{~ms}$ and $900 \mathrm{~ms}$ post onset: in our study, the participants were told that they would see familiar English words, novel names trained in the course of the experiment and words that they might not know. Consequently, having encountered a stimulus they were not familiar with (low recognition confidence), they may have tried to remember if this stimulus had been presented earlier by consciously retrieving episode-specific information associated with the training sessions (i.e., recollection). Nevertheless, while this interpretation is intuitively appealing, it must be taken with caution because the temporal and the spatial distributions of the observed effects overlap with both the FN400 (400-600 ms post onset at frontal and central sites) and the LPC (500-800 ms at parietal sites) components. Further research involving paradigms such as source memory and item recognition tests is required to separate the involvement of familiarity and recollection processes to item recognition.

To conclude, our findings suggest that both 0-day and 1-day consolidation periods may have been insufficient in this study for the integration of novel names for novel concepts into semantic memory. We also found evidence that the electrophysiological responses to the recent novel names were distinct from those to the nonwords and those to the remote novel names. We took the latter effect to reflect differences in familiarity and recollection across the two sets of novel names due to differences in recency of exposure. Taken together, we propose that, immediately 
after exposure to novel names (recent set), the system can only rely on episodic memory to distinguish these names from those acquired one day earlier (remote set) or previously unseen stimuli (nonwords). Because we used a training paradigm in which learning was passive, it would be interesting to see whether our findings replicate in paradigms that aim to explicitly facilitate learning (e.g., paradigms where learning is interleaved with testing or spaced learning, see Bjork \& Kroll, 2015; Cepeda et al., 2006, for reviews). Future research could also investigate whether our findings can be generalised to learning of novel names for familiar concepts.

\section{CRediT Author statement}

Maria Korochkina: Conceptualisation, methodology, software, validation, formal analysis, investigation, data curation, writing (original draft), writing (review and editing), visualisation. Lyndsey Nickels: Conceptualisation, methodology, validation, writing (review and editing), supervision.

Audrey Bürki: Conceptualisation, methodology, validation, writing (review and editing), supervision.

\section{Acknowledgements}

The authors would like to thank Andrew Roberts for his help in recording the stimuli, Nicholas Cooney and Ryssa Moffat for their help in the pilot phase and with participant recruitment, and Robert Taferner for his help in preparation of the visual elements for the Methods section.

\section{Funding}

MK was supported by a PhD stipend from Macquarie University [CTiMQRES IDEALAB; Allocation №2019129]. Costs for participant reimbursement were covered by the Cognitive Science Postgraduate Grant (CPGR4 2020) to MK. LN received support from an Australian Research Council Discovery Project Grant [DP190101490]. Funding sources had no involvement in study design, collection, analysis and interpretation of data, in the writing of the report or decision to submit the article for publication. 


\section{Appendices}

\section{Appendix A Matching of stimuli for different psycholinguistic variables: Model output}

A.1 Lists for the learning phase

OLD20 for Set 1 and Set 2

\begin{tabular}{lccccccc}
\hline & Mean & Median & Mode & $\begin{array}{c}95 \% \text { HDI } \\
\text { lower }\end{array}$ & $\begin{array}{c}95 \% \text { HDI } \\
\text { upper }\end{array}$ & $\begin{array}{c}\text { Comp. } \\
\text { value }\end{array}$ & $\begin{array}{r}\%> \\
\text { Comp. } \\
\text { value }\end{array}$ \\
\hline$\mu_{1}$ & 2.024 & 2.024 & 2.0313 & 1.766 & 2.276 & & \\
$\mu_{2}$ & 2.135 & 2.135 & 2.137 & 1.880 & 2.396 & & \\
$\mu_{\text {diff }}$ & -0.111 & -0.111 & -0.106 & -0.471 & 0.244 & 0 & 26.800 \\
$\sigma_{1}$ & 0.540 & 0.530 & 0.509 & 0.346 & 0.772 & & \\
$\sigma_{2}$ & 0.551 & 0.538 & 0.516 & 0.372 & 0.760 & & \\
$\sigma_{\text {diff }}$ & -0.010 & -0.010 & -0.007 & -0.307 & 0.274 & 0 & 47.000 \\
$\nu$ & 35.671 & 27.094 & 11.269 & 1.350 & 95.206 & & \\
$\log _{10} \nu$ & 1.406 & 1.433 & 1.533 & 0.662 & 2.103 & & \\
effect size & -0.207 & -0.204 & -0.176 & -0.871 & 0.433 & 0 & 26.800 \\
\hline
\end{tabular}

Table 12. Bayesian estimation for OLD20 for the two sets of novel names. 


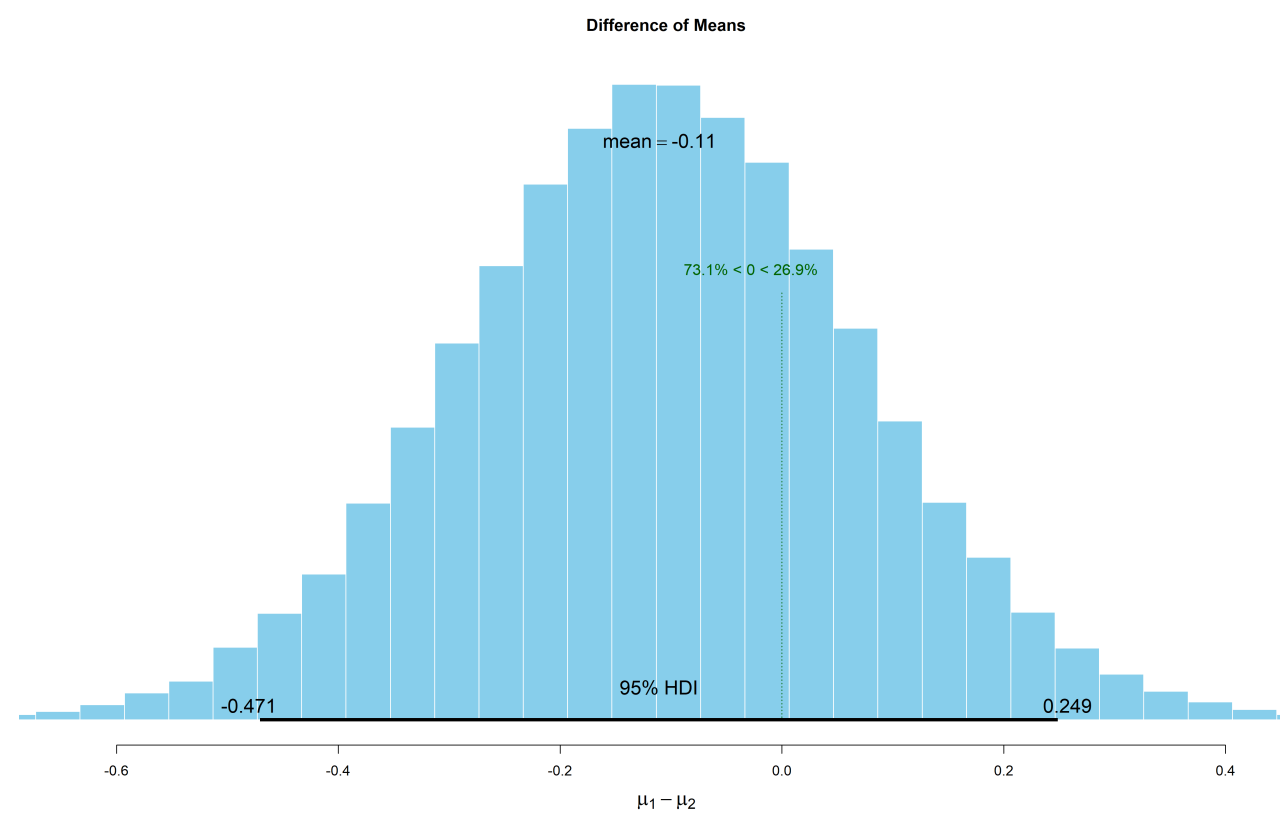

Figure 17. Posterior distribution of the difference in means for OLD20 for the two sets of novel names.

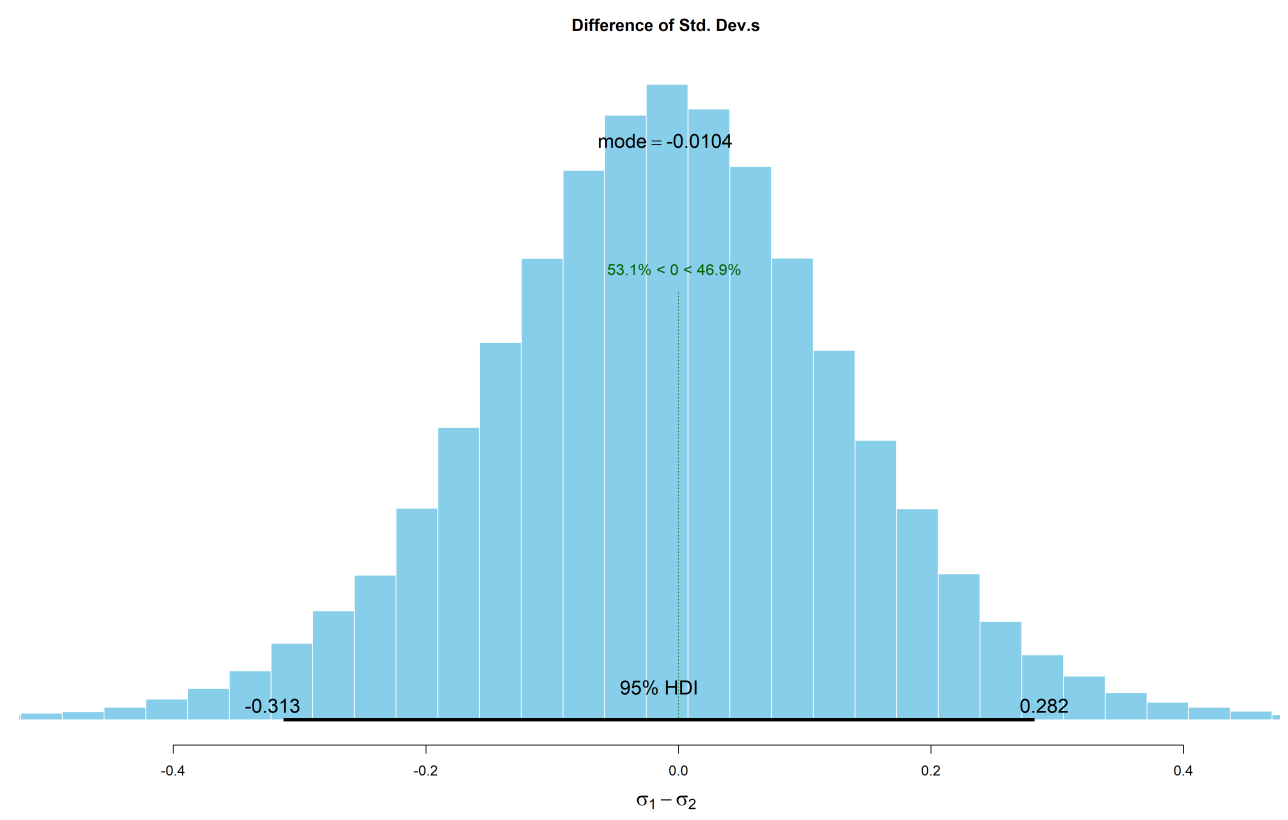

Figure 18. Posterior distribution of the difference in standard deviation for OLD20 for the two sets of novel names. 


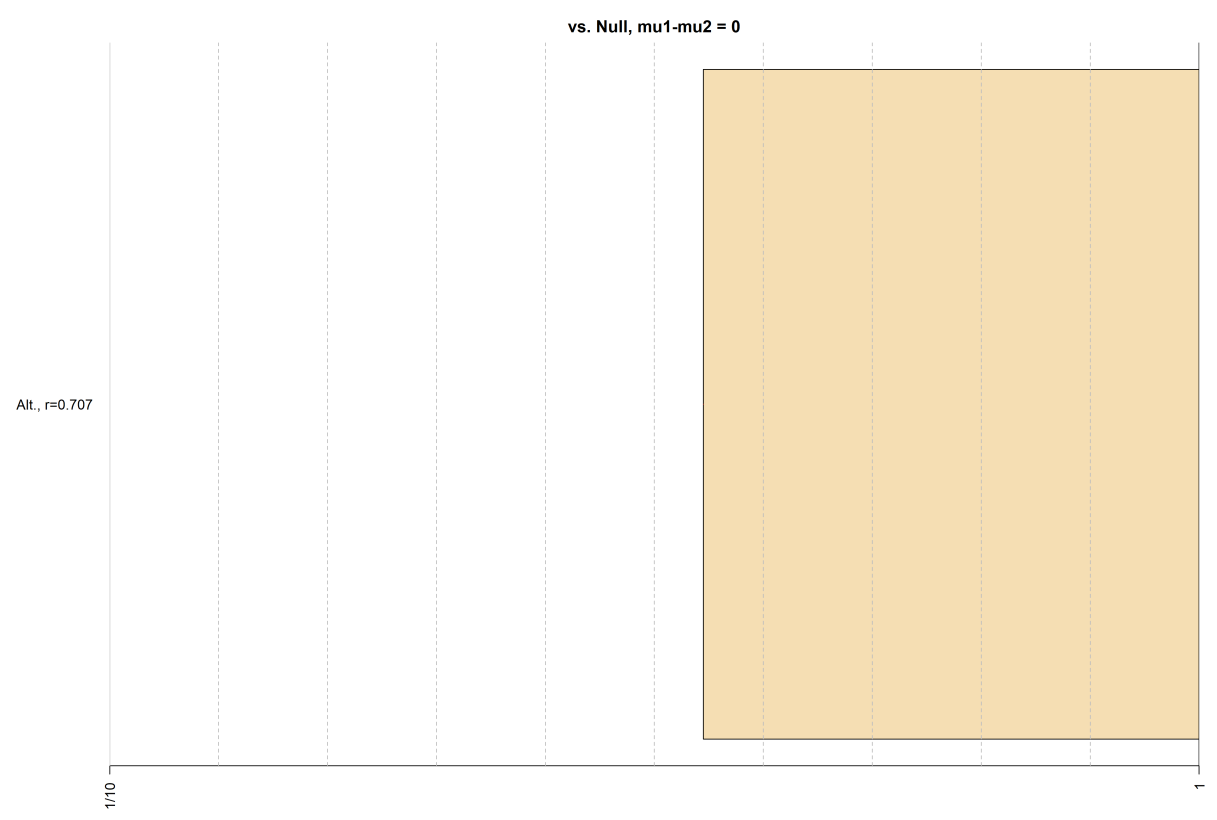

Figure 19. Graphical representation of the Bayes factor (denominator model: $\mu_{1}-\mu_{2}=$ $0)$.
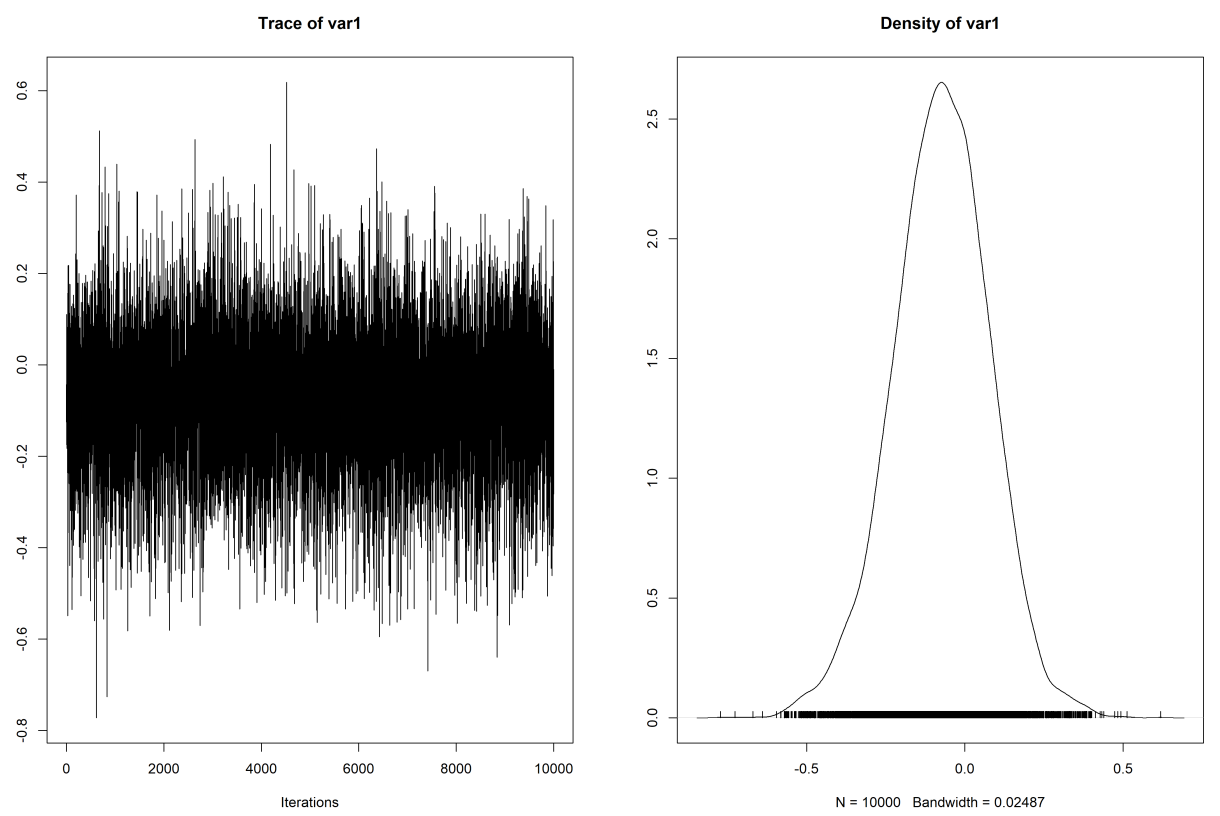

Figure 20. Sampling diagnostics of the posterior for the numerator model. 


\begin{tabular}{lccccccc}
\hline & Mean & Median & Mode & $\begin{array}{c}95 \% \text { HDI } \\
\text { lower }\end{array}$ & $\begin{array}{c}95 \% \text { HDI } \\
\text { upper }\end{array}$ & $\begin{array}{c}\text { Comp. } \\
\text { value }\end{array}$ & $\begin{array}{r}\text { Comp. } \\
\text { value }\end{array}$ \\
\hline$\mu_{1}$ & 5.307 & 5.308 & 5.324 & 4.755 & 5.854 & & \\
$\mu_{2}$ & 5.403 & 5.404 & 5.427 & 4.765 & 6.035 & & \\
$\mu_{\text {diff }}$ & -0.096 & -0.096 & -0.080 & -0.943 & 0.726 & 0 & 40.600 \\
$\sigma_{1}$ & 1.177 & 1.151 & 1.092 & 0.789 & 1.617 & & \\
$\sigma_{2}$ & 1.358 & 1.327 & 1.249 & 0.903 & 1.882 & & \\
$\sigma_{d i f f}$ & -0.181 & -0.174 & -0.169 & -0.852 & 0.476 & 0 & 28.500 \\
$\nu$ & 37.633 & 29.204 & 13.610 & 2.372 & 97.955 & & \\
$\log _{10} \nu$ & 1.444 & 1.465 & 1.521 & 0.758 & 2.102 & & \\
effect size & -0.076 & -0.076 & -0.059 & -0.724 & 0.566 & 0 & 40.600 \\
\hline
\end{tabular}

Table 13. Bayesian estimation for number of letters for the two sets of novel names.

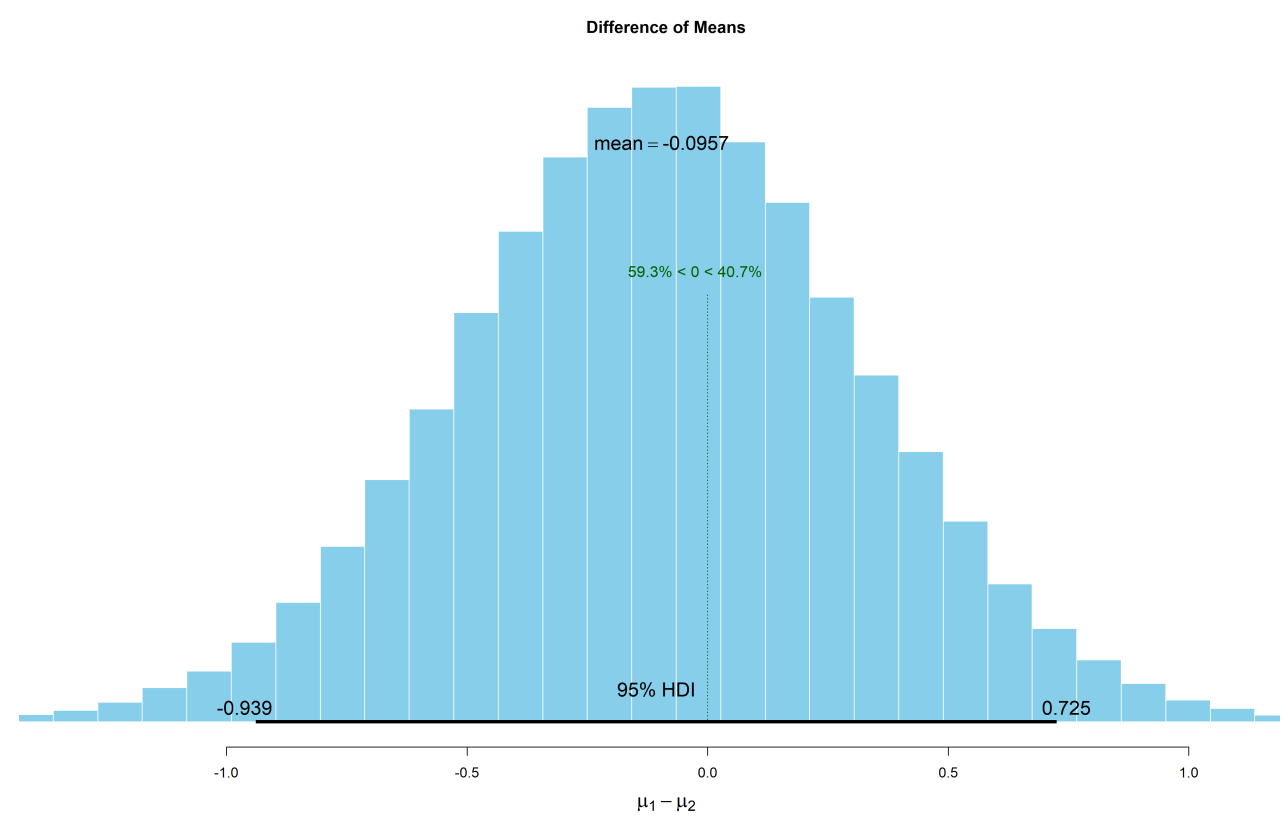

Figure 21. Posterior distribution of the difference in means for number of letters for the two sets of novel names. 


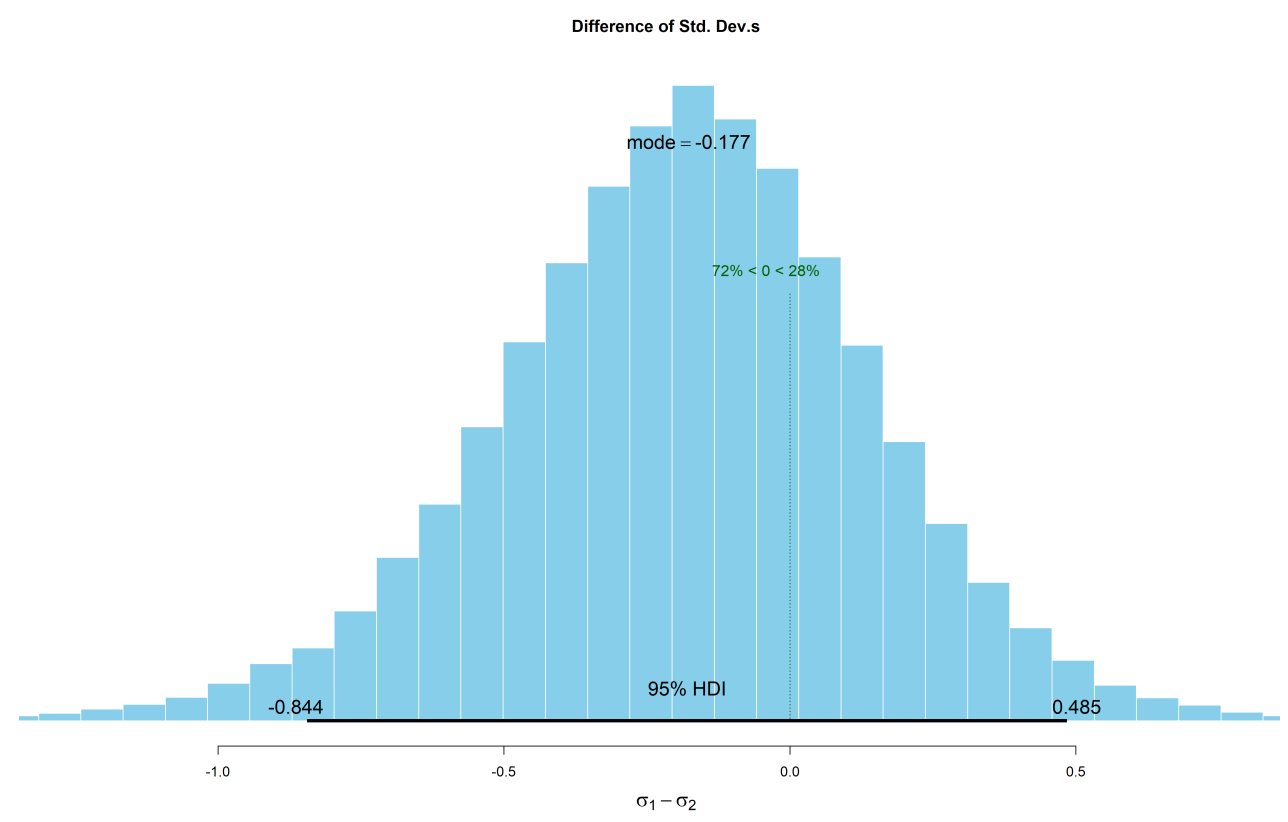

Figure 22. Posterior distribution of the difference in standard deviation for number of letters for the two sets of novel names.

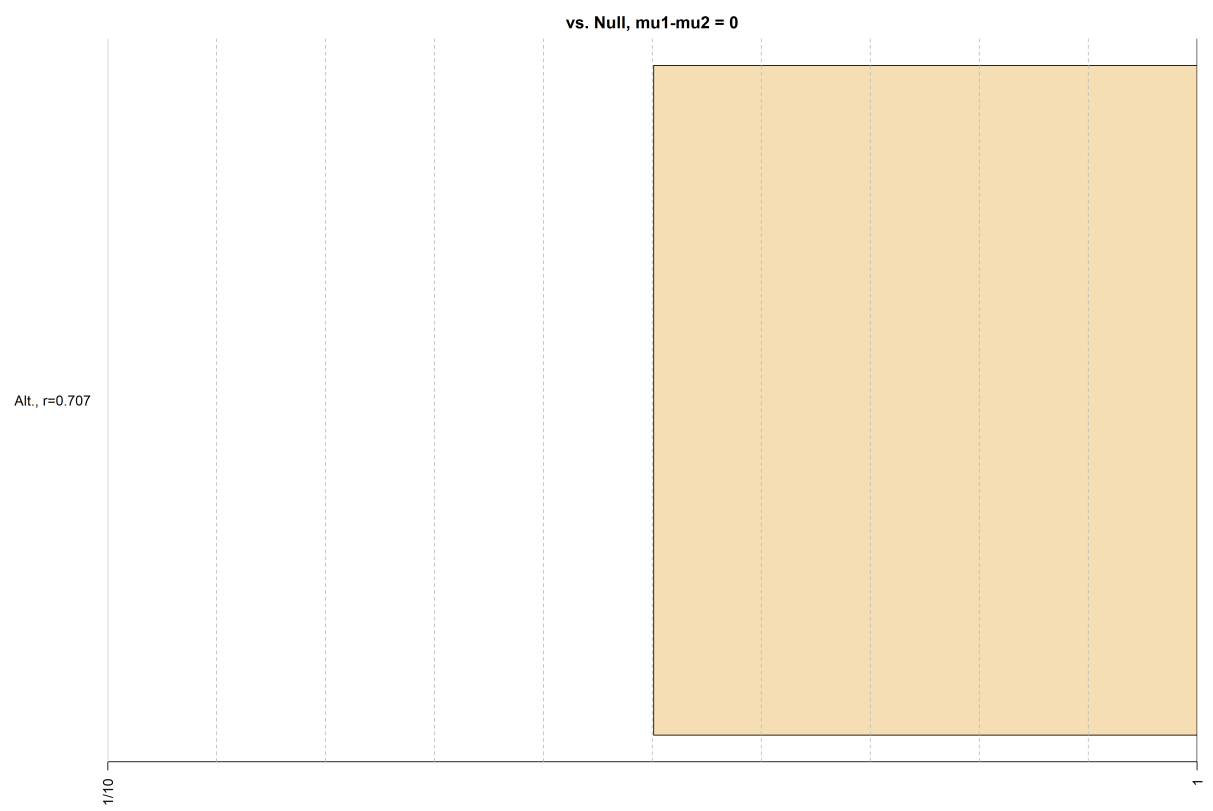

Figure 23. Graphical representation of the Bayes factor (denominator model: $\mu_{1}-\mu_{2}=$ $0)$. 

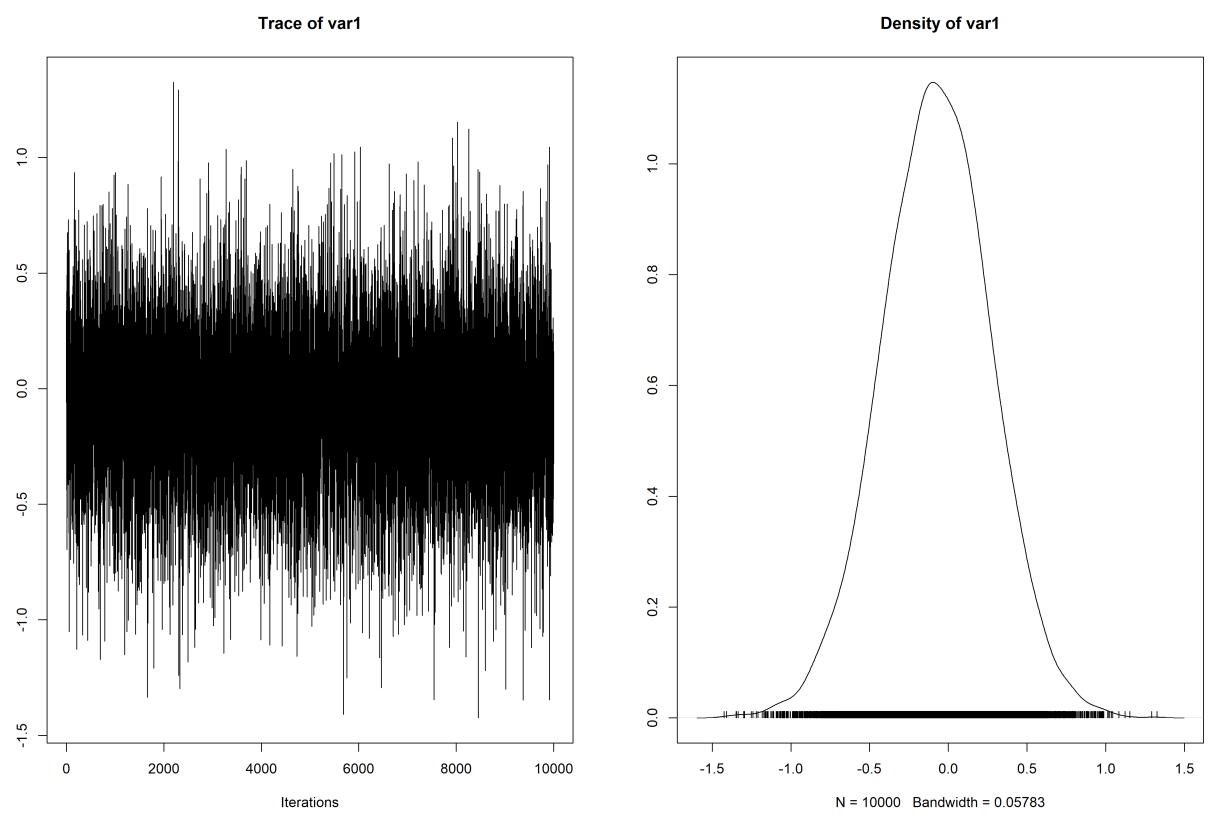

Figure 24. Sampling diagnostics of the posterior for the numerator model.

\section{Unconstrained bigram frequency for Set 1 and Set 2}

\begin{tabular}{lccccccr}
\hline & Mean & Median & Mode & $\begin{array}{c}95 \% \text { HDI } \\
\text { lower }\end{array}$ & $\begin{array}{c}95 \% \text { HDI } \\
\text { upper }\end{array}$ & $\begin{array}{c}\text { Comp. } \\
\text { value }\end{array}$ & $\begin{array}{r}\text { Comp. } \\
\text { value }\end{array}$ \\
\hline$\mu_{1}$ & 17089.713 & 17087.234 & 17063.433 & 12991.731 & 21156.009 & & \\
$\mu_{2}$ & 20138.858 & 20050.075 & 20049.744 & 12605.039 & 28186.790 & & \\
$\mu_{\text {diff }}$ & -3049.145 & -2979.277 & -2264.182 & -11893.654 & 5632.412 & 0 & 24.560 \\
$\sigma_{1}$ & 8557.988 & 8348.493 & 8007.909 & 5684.353 & 11832.657 & & \\
$\sigma_{2}$ & 15987.447 & 15698.093 & 15323.833 & 9275.418 & 23090.591 & & \\
$\sigma_{\text {diff }}$ & -7429.459 & -7207.325 & -6919.201 & -15321.575 & -320.630 & 0 & 1.690 \\
$\nu$ & 27.366 & 18.503 & 7.344 & 1.384 & 80.855 & & \\
$\log _{10} \nu$ & 1.264 & 1.267 & 1.340 & 0.531 & 2.009 & & \\
effect & -0.234 & -0.236 & -0.213 & -0.894 & 0.448 & 0 & 24.560 \\
size & & & & & & & \\
\hline
\end{tabular}

Table 14. Bayesian estimation for unconstrained bigram frequency for the two sets of novel names. 


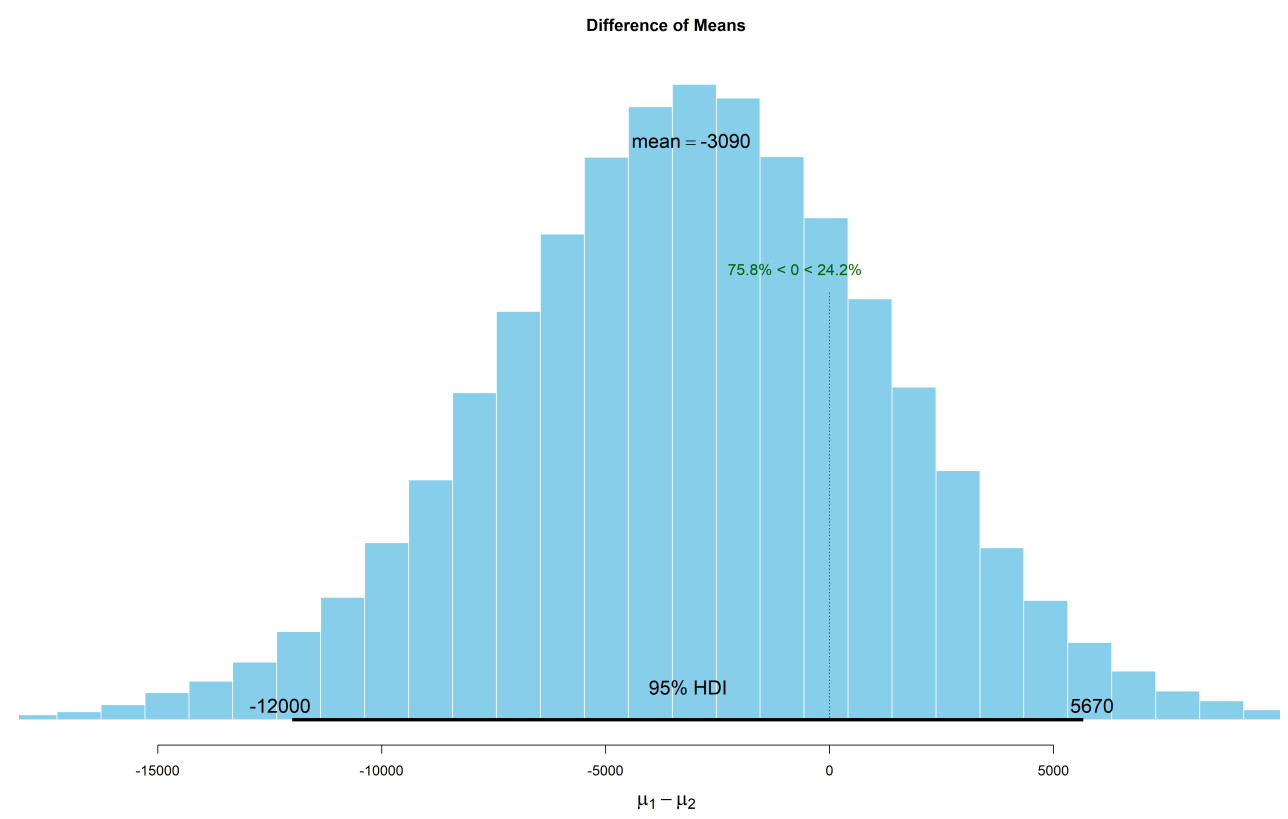

Figure 25. Posterior distribution of the difference in means for unconstrained bigram frequency for the two sets of novel names.

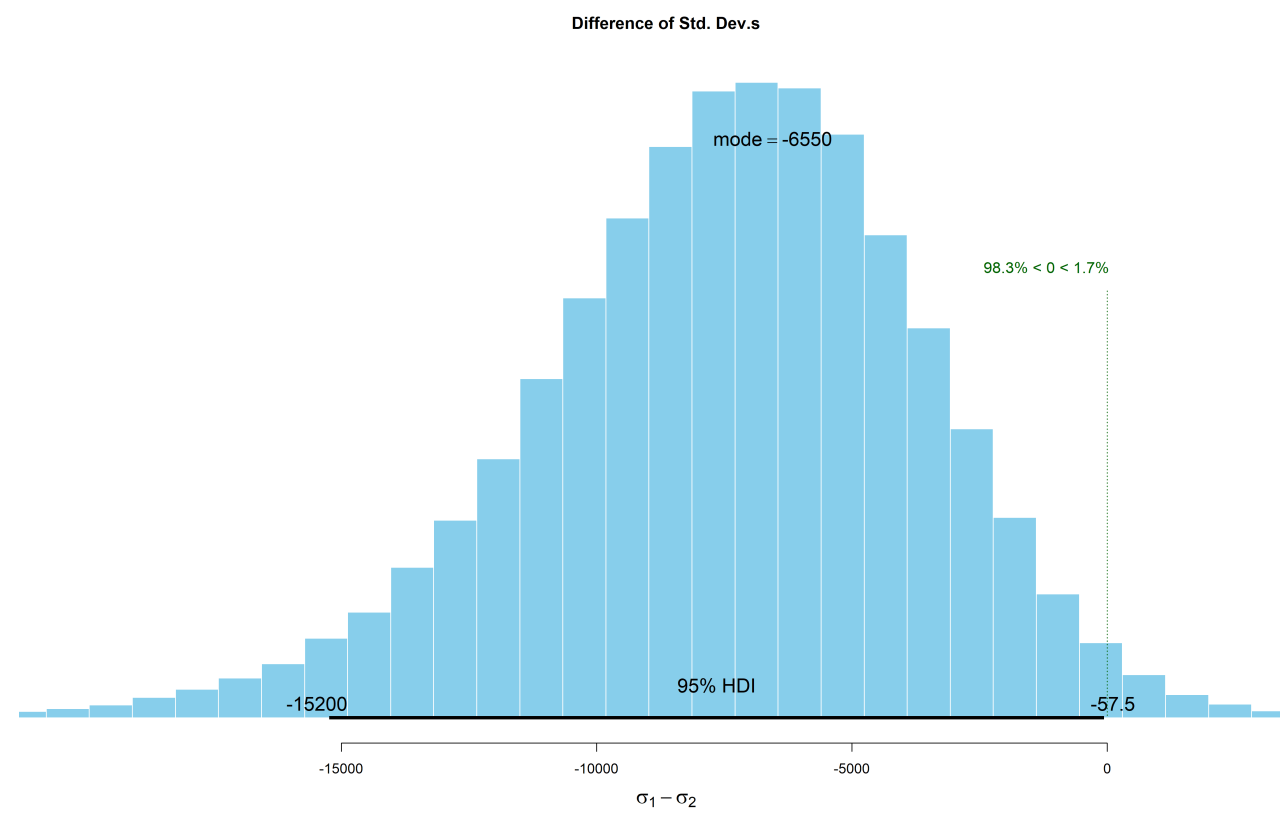

Figure 26. Posterior distribution of the difference in standard deviation for unconstrained bigram frequency for the two sets of novel names. 


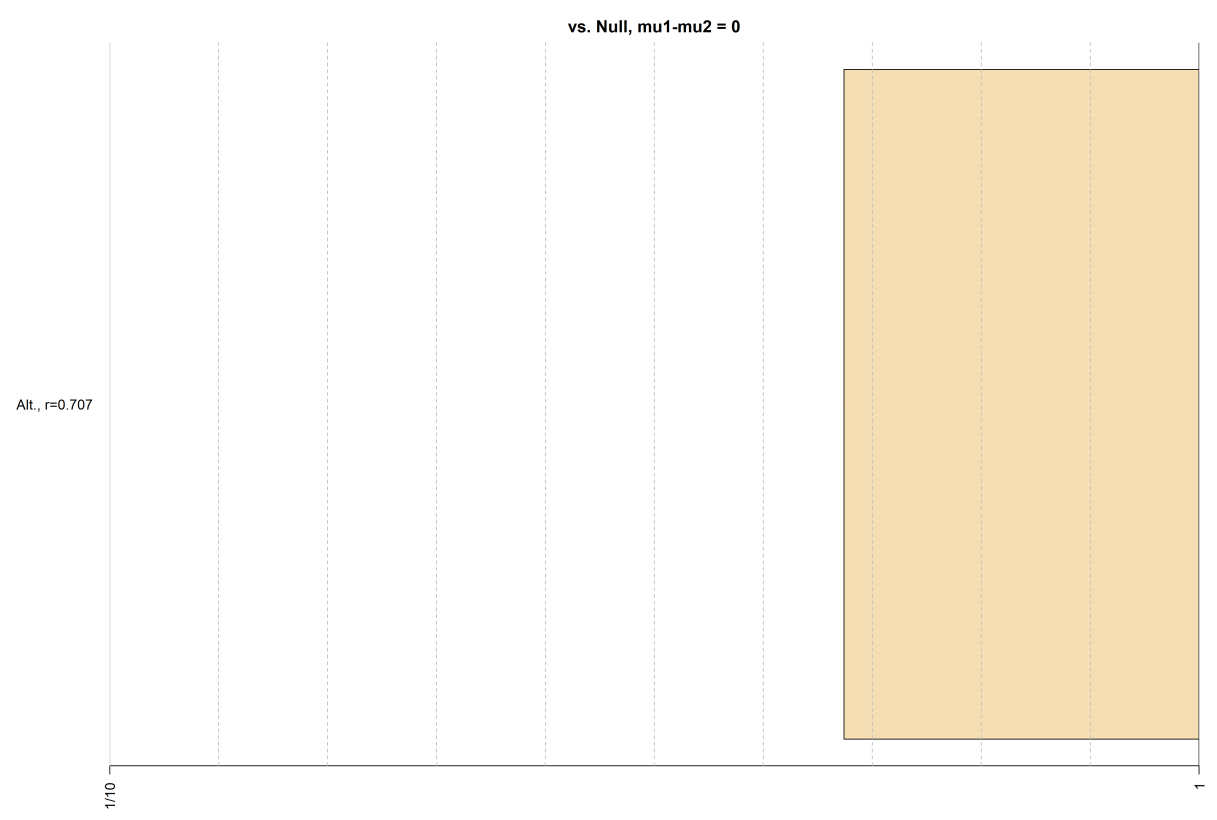

Figure 27. Graphical representation of the Bayes factor (denominator model: $\mu_{1}-\mu_{2}=$ $0)$.
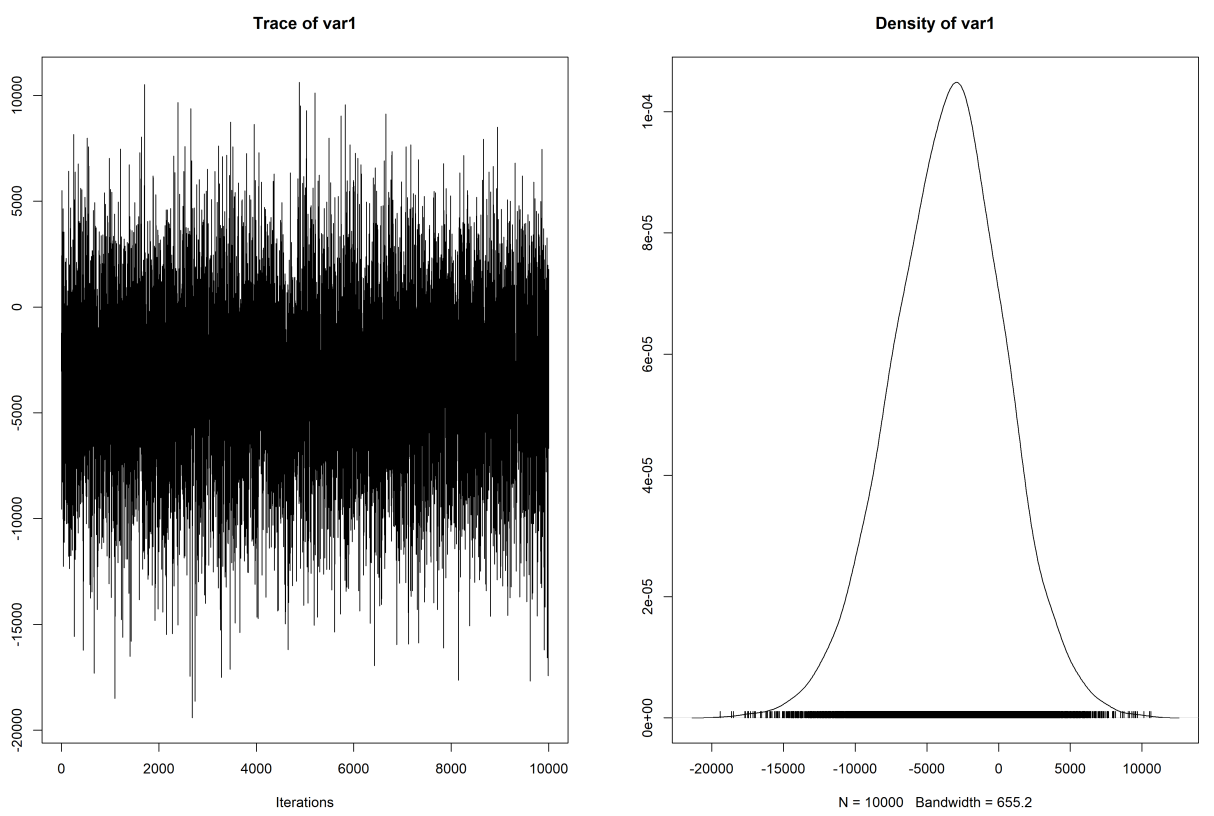

Figure 28. Sampling diagnostics of the posterior for the numerator model. 
Constrained bigram frequency for Set 1 and Set 2

\begin{tabular}{lccccccr}
\hline & Mean & Median & Mode & $\begin{array}{c}95 \% \text { HDI } \\
\text { lower }\end{array}$ & $\begin{array}{c}95 \% \text { HDI } \\
\text { upper }\end{array}$ & $\begin{array}{c}\text { Comp. } \\
\text { value }\end{array}$ & $\begin{array}{r}\%> \\
\text { Comp. } \\
\text { value }\end{array}$ \\
\hline$\mu_{1}$ & 641.375 & 632.355 & 618.826 & 412.515 & 887.386 & & \\
$\mu_{2}$ & 461.885 & 459.283 & 456.293 & 252.805 & 666.298 & & \\
$\mu_{\text {diff }}$ & 179.490 & 174.642 & 169.840 & -134.289 & 485.341 & 0 & 88.500 \\
$\sigma_{1}$ & 398.637 & 376.701 & 335.623 & 172.198 & 671.040 & & \\
$\sigma_{2}$ & 362.504 & 337.126 & 300.418 & 142.891 & 627.864 & & \\
$\sigma_{\text {diff }}$ & 36.132 & 35.817 & 38.115 & -311.786 & 387.465 & 0 & 59.500 \\
$\nu$ & 1.545 & 1.413 & 1.153 & 1.000 & 2.460 & & \\
$\log _{10} \nu$ & 0.170 & 0.150 & 0.091 & 0.000 & 0.391 & & \\
effect size & 0.482 & 0.472 & 0.468 & -0.306 & 1.301 & 0 & 88.500 \\
\hline
\end{tabular}

Table 15. Bayesian estimation for constrained bigram frequency for the two sets of novel names.

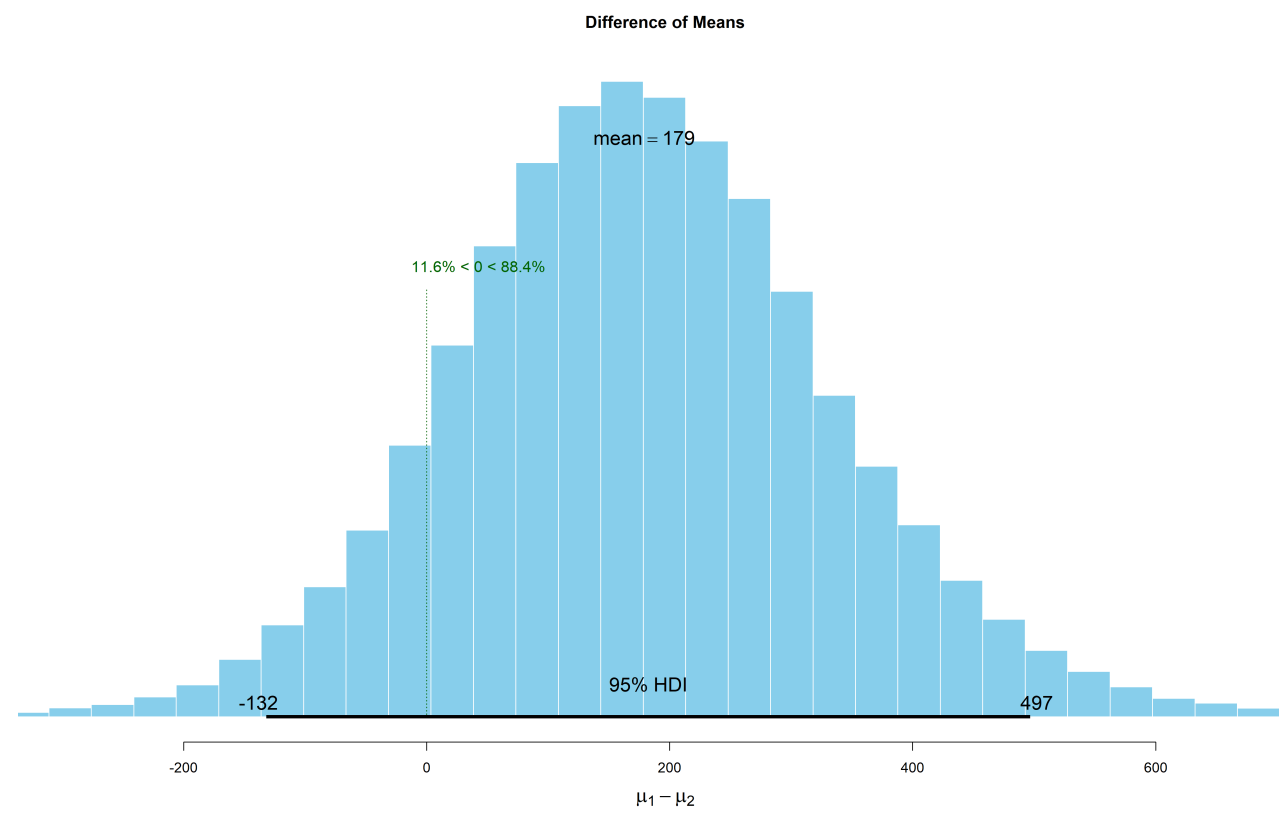

Figure 29. Posterior distribution of the difference in means for constrained bigram frequency for the two sets of novel names. 


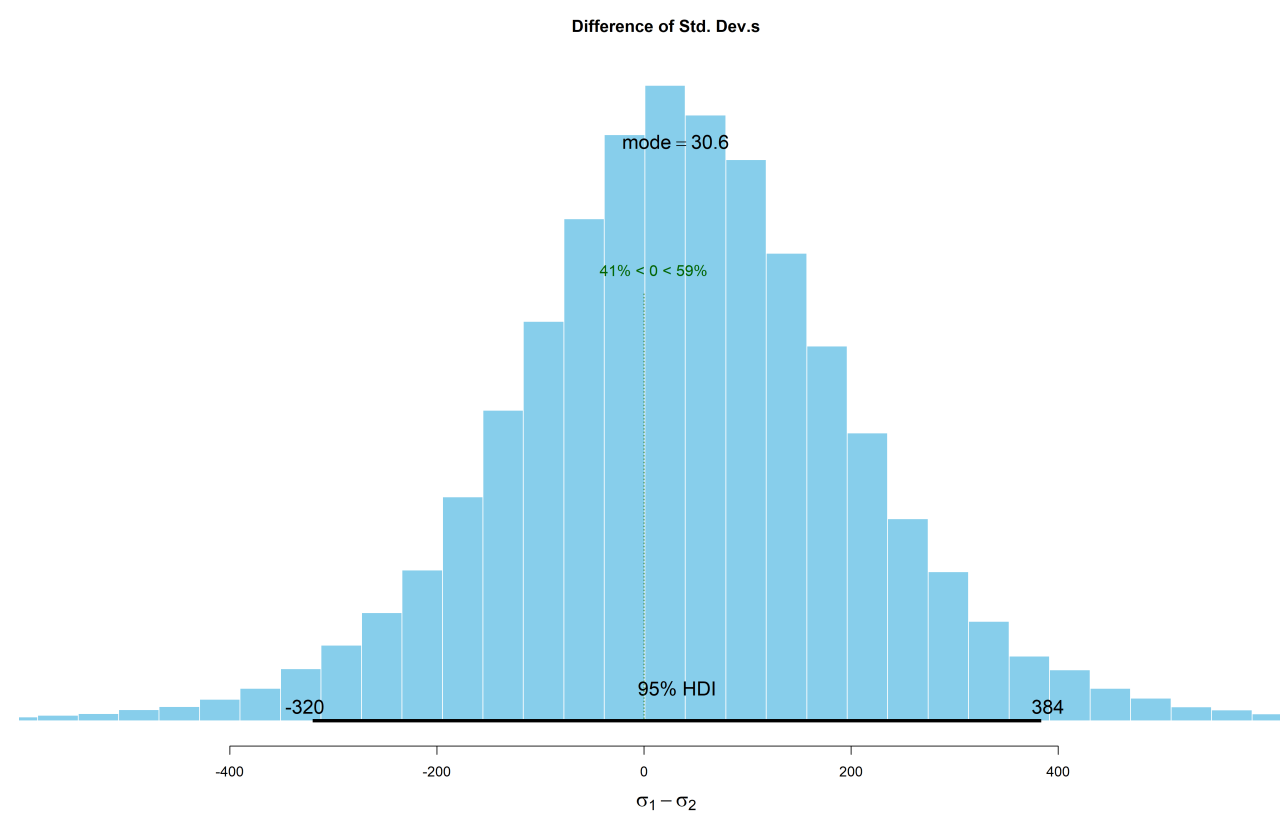

Figure 30. Posterior distribution of the difference in standard deviation for constrained bigram frequency for the two sets of novel names.

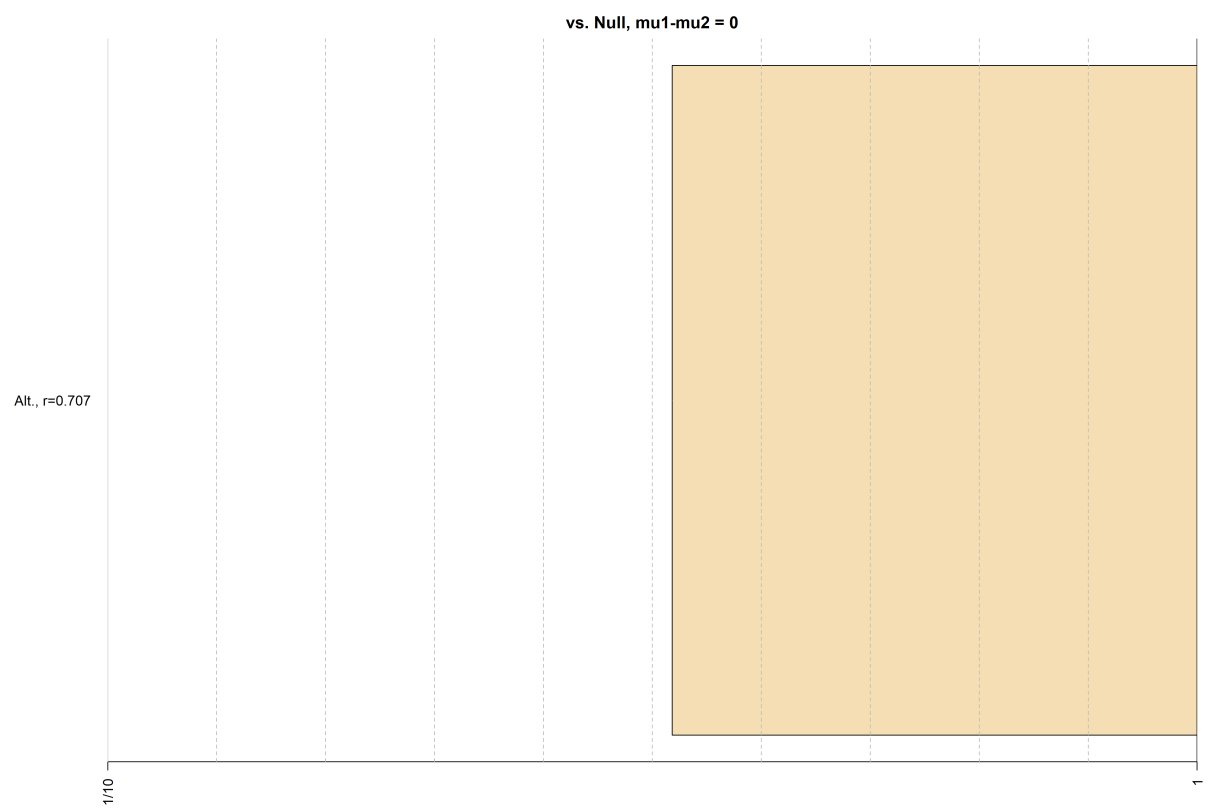

Figure 31. Graphical representation of the Bayes factor (denominator model: $\mu_{1}-\mu_{2}=$ $0)$. 

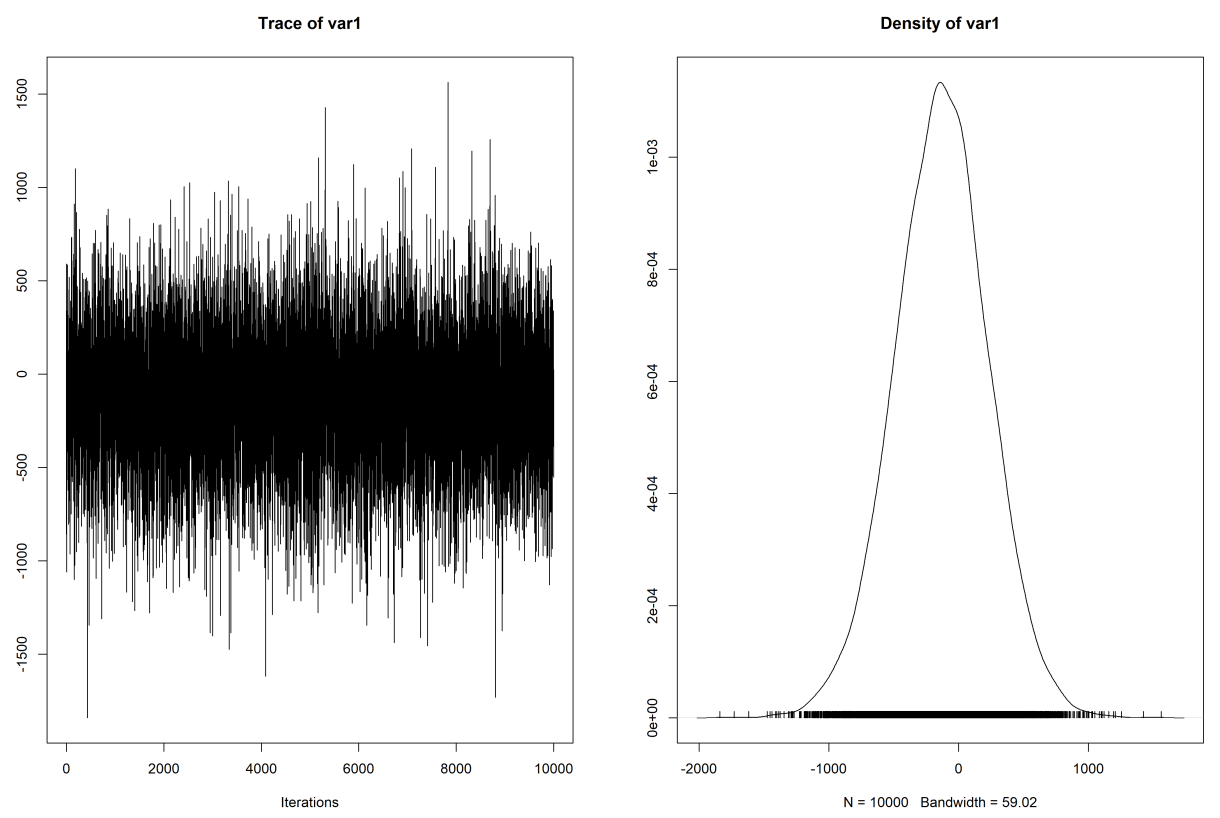

Figure 32. Sampling diagnostics of the posterior for the numerator model.

\section{A.2 Lists for the primed continuous lexical decision task}

OLD20 for the trained novel names and pseudoword fillers

\begin{tabular}{lccccccc}
\hline & Mean & Median & Mode & $\begin{array}{c}95 \% \text { HDI } \\
\text { lower }\end{array}$ & $\begin{array}{c}95 \% \text { HDI } \\
\text { upper }\end{array}$ & $\begin{array}{c}\text { Comp. } \\
\text { value }\end{array}$ & $\begin{array}{r}\%> \\
\text { Comp. } \\
\text { value }\end{array}$ \\
\hline$\mu_{1}$ & 2.394 & 2.394 & 2.392 & 2.301 & 2.487 & & \\
$\mu_{2}$ & 2.339 & 2.338 & 2.336 & 2.152 & 2.525 & & \\
$\mu_{\text {diff }}$ & 0.055 & 0.0554 & 0.062 & -0.153 & 0.264 & 0 & 70.300 \\
$\sigma_{1}$ & 0.581 & 0.579 & 0.577 & 0.514 & 0.652 & & \\
$\sigma_{2}$ & 0.575 & 0.569 & 0.566 & 0.444 & 0.718 & & \\
$\sigma_{\text {diff }}$ & 0.005 & 0.012 & 0.020 & -0.152 & 0.154 & 0 & 55.400 \\
$\nu$ & 49.381 & 41.192 & 25.489 & 7.649 & 113.513 & & \\
$\log _{10} \nu$ & 1.611 & 1.615 & 1.642 & 1.083 & 2.127 & & \\
effect size & 0.096 & 0.096 & 0.104 & -0.263 & 0.451 & 0 & 70.300 \\
\hline
\end{tabular}

Table 16. Bayesian estimation for OLD20 for the trained novel names and pseudoword filles. 


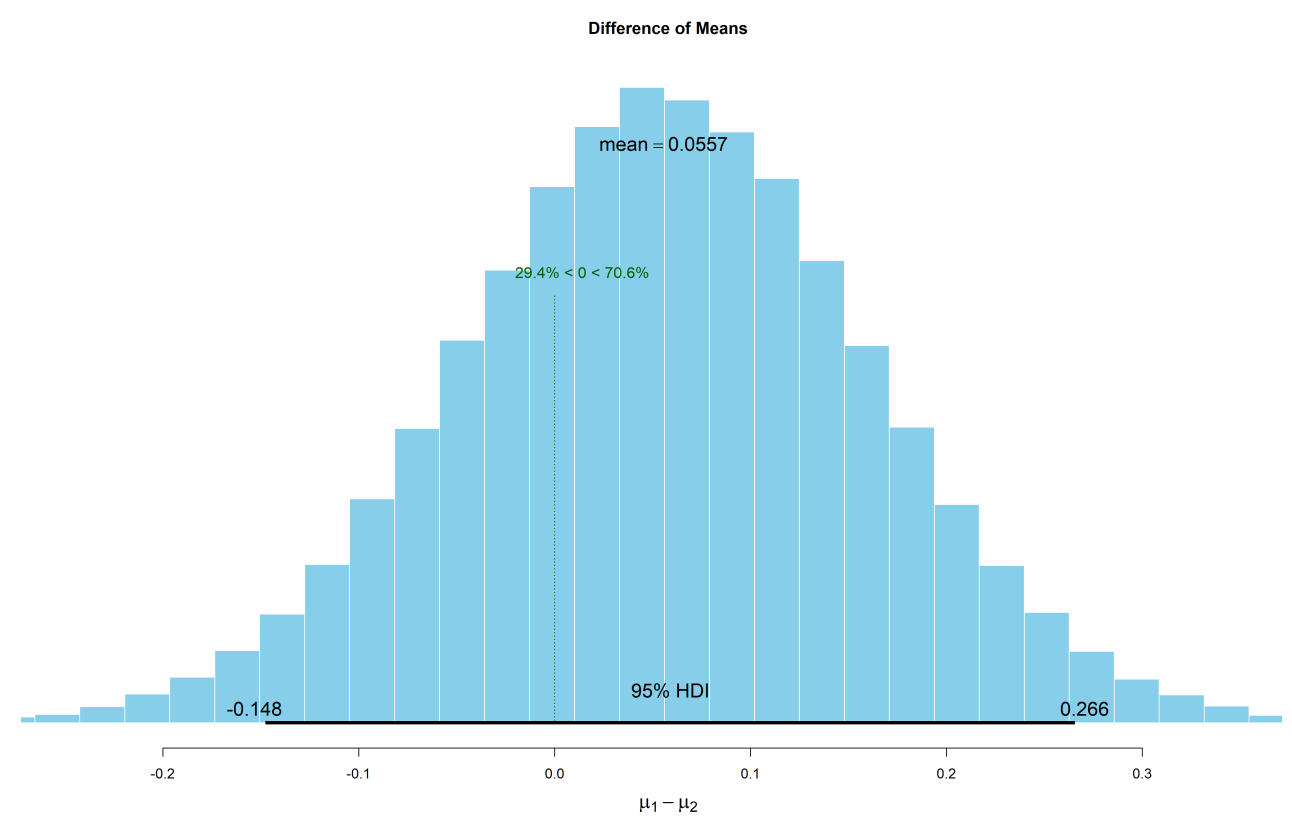

Figure 33. Posterior distribution of the difference in means for OLD20 for the trained novel names and pseudoword filles.

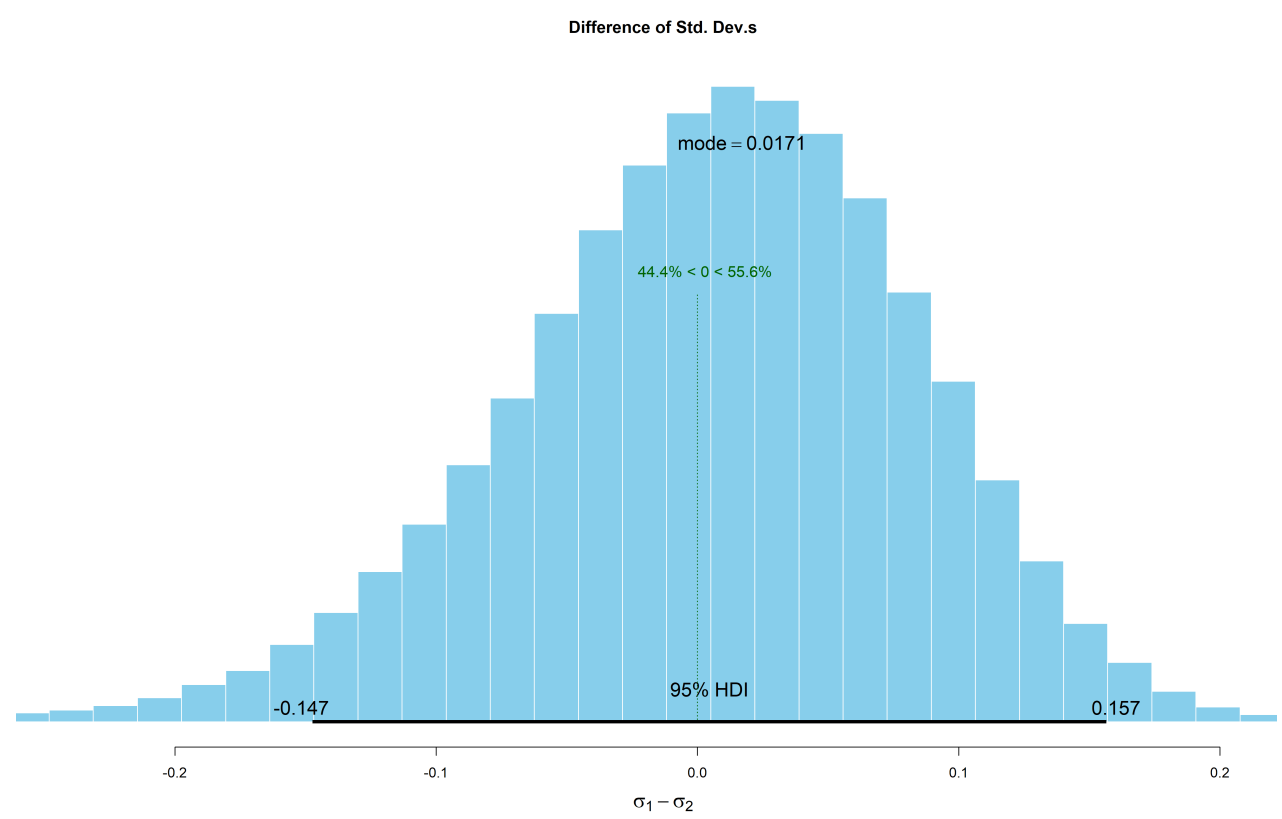

Figure 34. Posterior distribution of the difference in standard deviation for OLD20 for the trained novel names and pseudoword filles. 


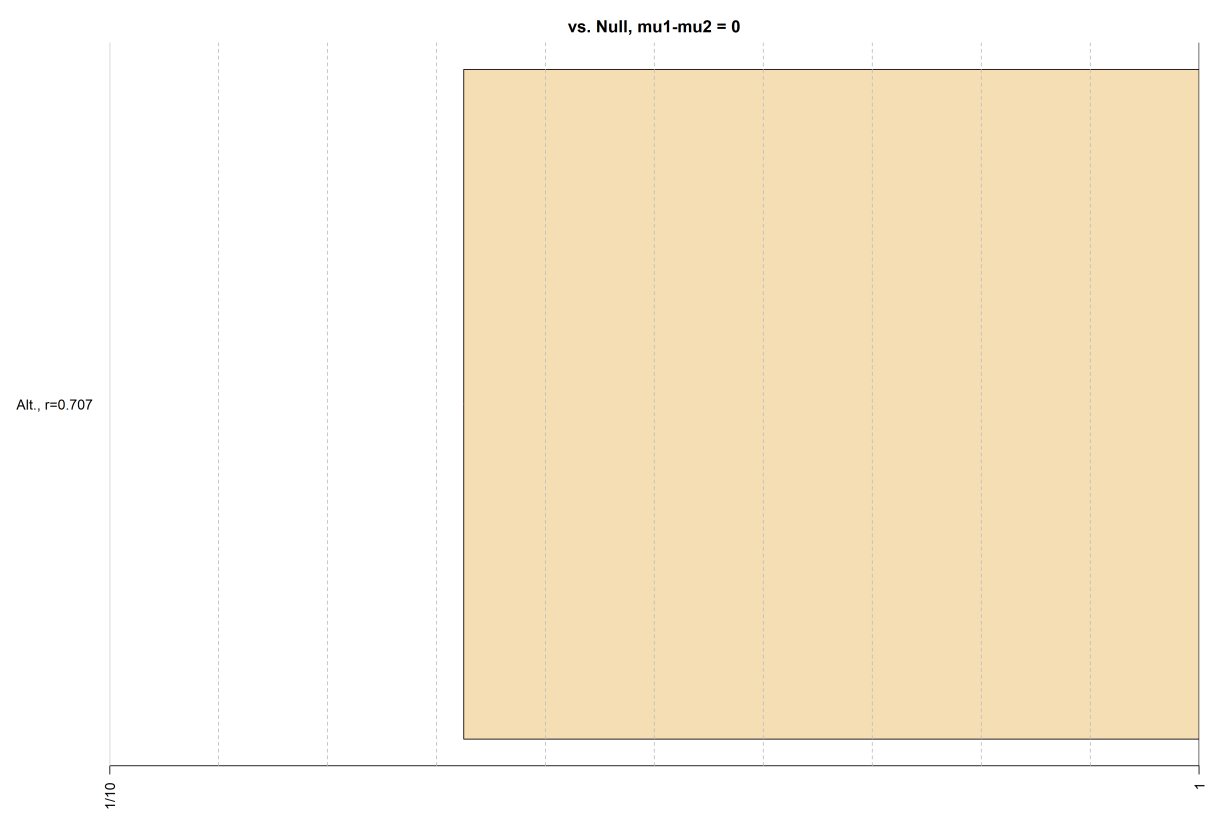

Figure 35. Graphical representation of the Bayes factor (denominator model: $\mu_{1}-\mu_{2}=$ $0)$.
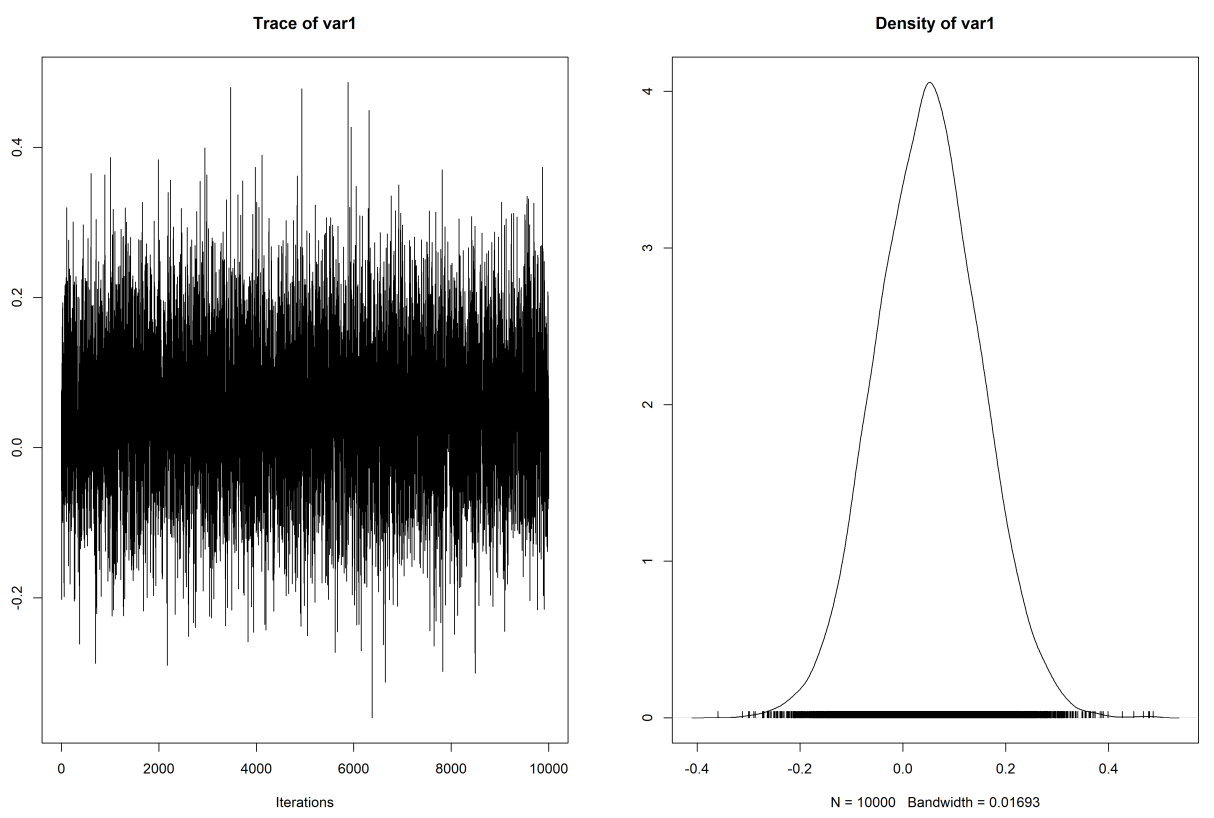

Figure 36. Sampling diagnostics of the posterior for the numerator model. 
Number of letters for the trained novel names and pseudoword fillers

\begin{tabular}{lccccccc}
\hline & Mean & Median & Mode & $\begin{array}{c}95 \% \text { HDI } \\
\text { lower }\end{array}$ & $\begin{array}{c}95 \% \text { HDI } \\
\text { upper }\end{array}$ & $\begin{array}{c}\text { Comp. } \\
\text { value }\end{array}$ & $\begin{array}{r}\%> \\
\text { Comp. } \\
\text { value }\end{array}$ \\
\hline$\mu_{1}$ & 5.485 & 5.485 & 5.486 & 5.278 & 5.688 & & \\
$\mu_{2}$ & 5.353 & 5.352 & 5.329 & 4.959 & 5.743 & & \\
$\mu_{\text {diff }}$ & 0.132 & 0.132 & 0.106 & -0.319 & 0.565 & 0 & 72.300 \\
$\sigma_{1}$ & 1.298 & 1.295 & 1.288 & 1.154 & 1.451 & & \\
$\sigma_{2}$ & 1.228 & 1.214 & 1.191 & 0.953 & 1.531 & & \\
$\sigma_{\text {diff }}$ & 0.070 & 0.081 & 0.105 & -0.263 & 0.389 & 0 & 68.500 \\
$\nu$ & 54.969 & 47.122 & 30.564 & 9.543 & 121.399 & & \\
$\log _{10} \nu$ & 1.667 & 1.673 & 1.679 & 1.175 & 2.161 & & 72.300 \\
effect size & 0.105 & 0.105 & 0.079 & -0.244 & 0.448 & 0 & \\
\hline
\end{tabular}

Table 17. Bayesian estimation for number of letters for the trained novel names and pseudoword filles.

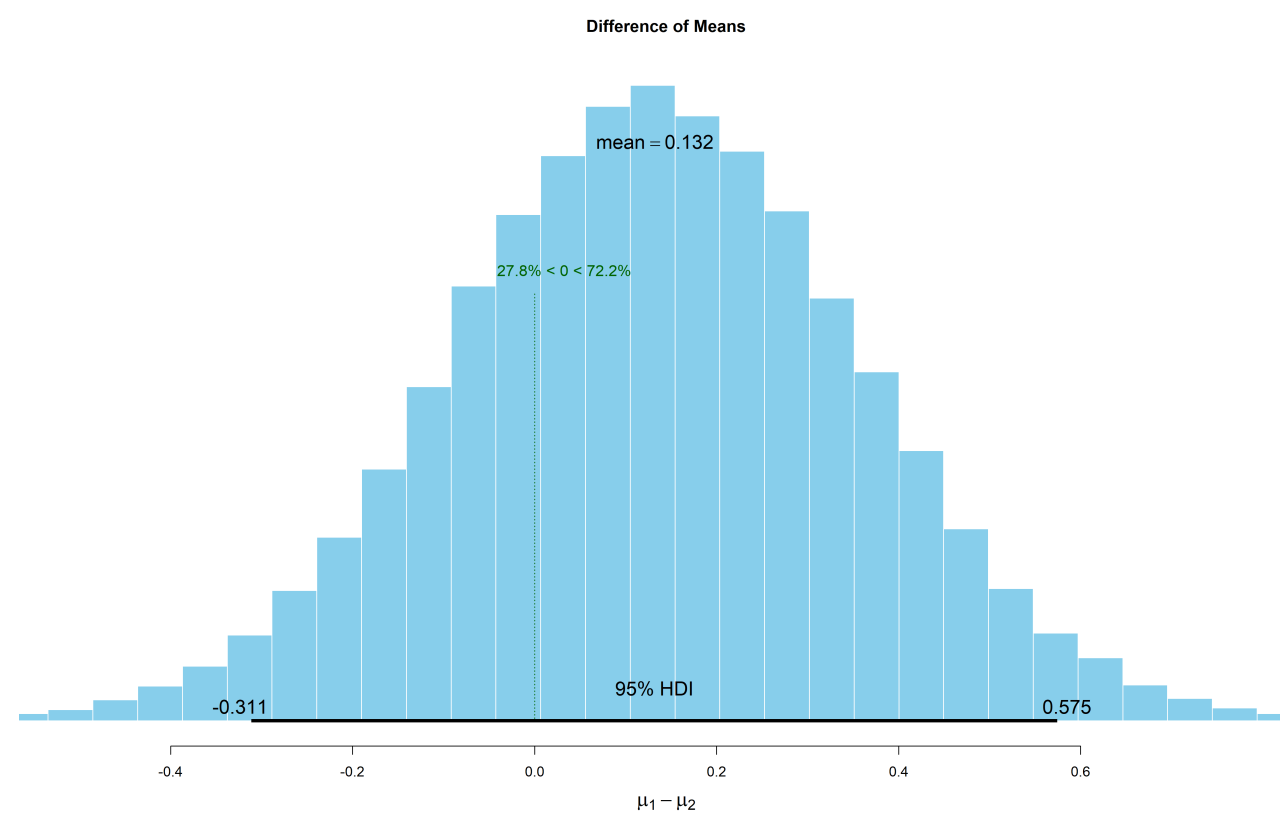

Figure 37. Posterior distribution of the difference in means for number of letters for the trained novel names and pseudoword filles. 


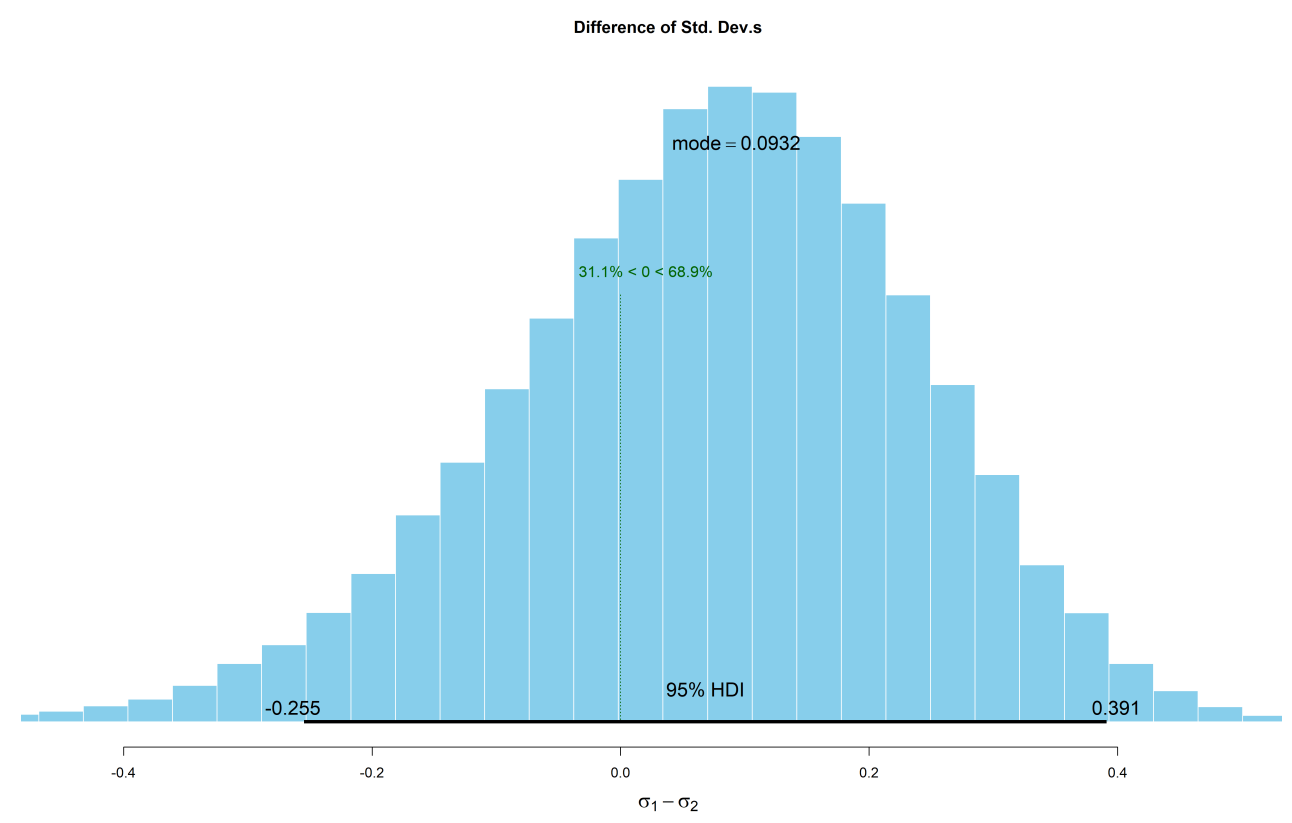

Figure 38. Posterior distribution of the difference in standard deviation for number of letters for the trained novel names and pseudoword filles.

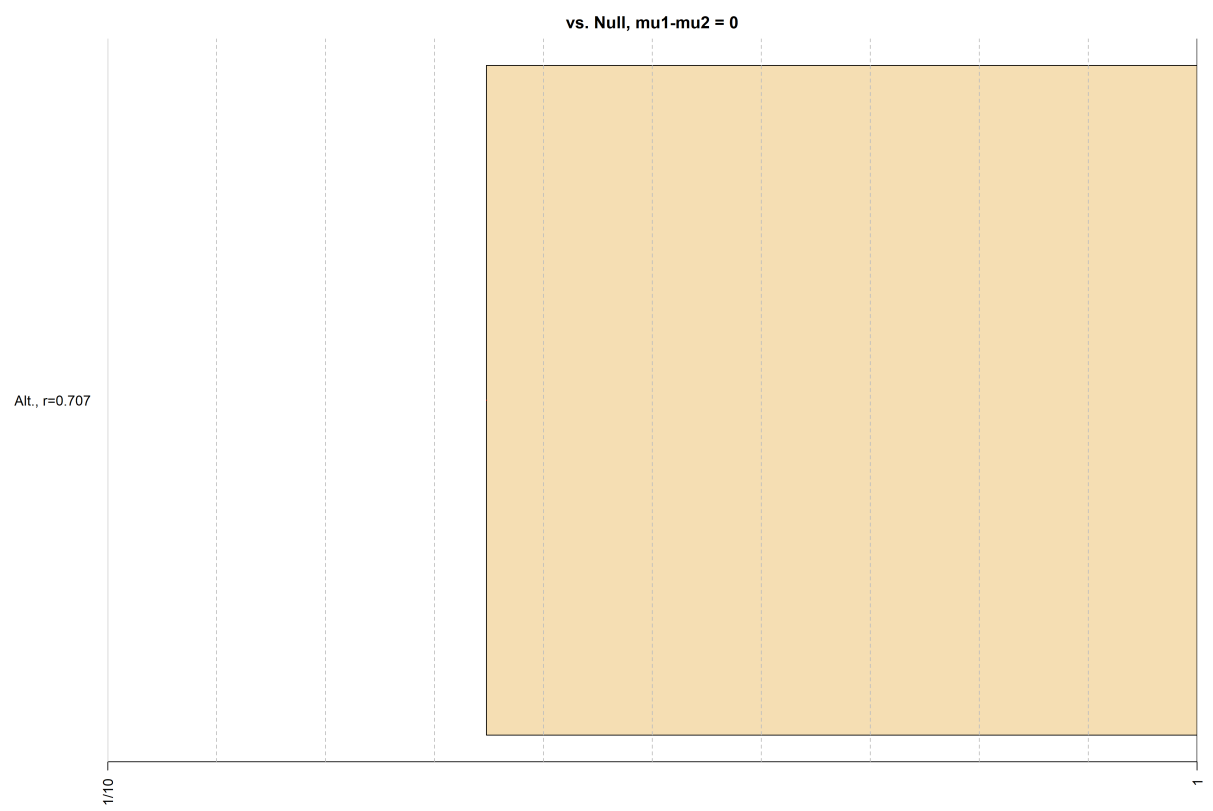

Figure 39. Graphical representation of the Bayes factor (denominator model: $\mu_{1}-\mu_{2}=$ $0)$. 

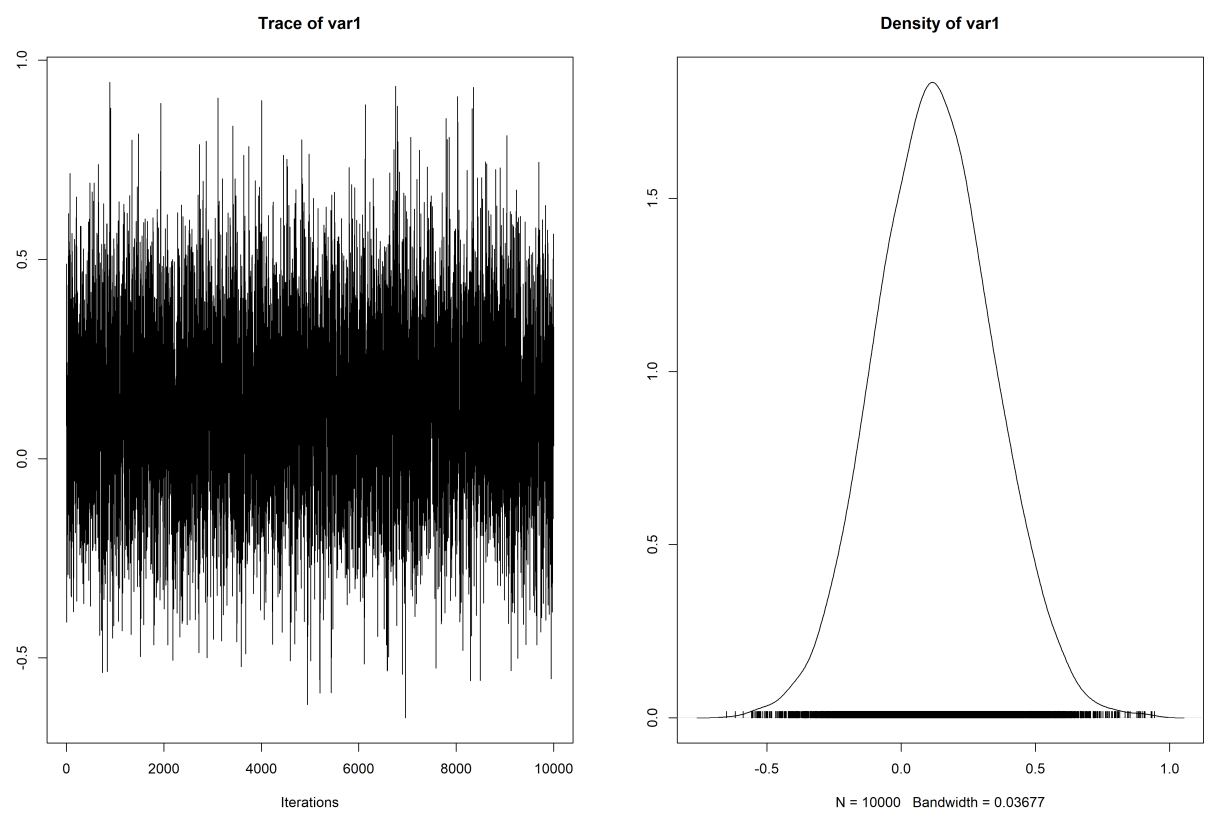

Figure 40. Sampling diagnostics of the posterior for the numerator model.

Unconstrained bigram frequency for the trained novel names and pseudoword filles

\begin{tabular}{lccccccc}
\hline & Mean & Median & Mode & $\begin{array}{c}95 \% \text { HDI } \\
\text { lower }\end{array}$ & $\begin{array}{c}95 \% \text { HDI } \\
\text { upper }\end{array}$ & $\begin{array}{c}\text { Comp. } \\
\text { value }\end{array}$ & $\begin{array}{r}\%> \\
\text { Comp. } \\
\text { value }\end{array}$ \\
\hline$\mu_{1}$ & 17843.797 & 17827.971 & 17815.380 & 16067.287 & 19689.561 & & \\
$\mu_{2}$ & 17138.850 & 17114.856 & 17329.136 & 13511.207 & 20656.300 & & \\
$\mu_{\text {diff }}$ & 704.948 & 732.017 & 809.084 & -3231.457 & 4634.539 & 0 & 64.200 \\
$\sigma_{1}$ & 9133.823 & 9094.107 & 9054.153 & 7346.776 & 10919.965 & & \\
$\sigma_{2}$ & 9301.128 & 9172.079 & 8808.636 & 6580.983 & 12318.284 & & \\
$\sigma_{\text {diff }}$ & -167.305 & -62.193 & 120.949 & -3406.664 & 2901.172 & 0 & 48.400 \\
$\nu$ & 3.515 & 3.345 & 3.053 & 1.958 & 5.423 & & \\
$\log _{10} \nu$ & 0.531 & 0.524 & 0.499 & 0.320 & 0.751 & & \\
effect & 0.078 & 0.080 & 0.091 & -0.349 & 0.504 & 0 & 64.200 \\
size & & & & & & & \\
\hline
\end{tabular}

Table 18. Bayesian estimation for unconstrained bigram frequency for the trained novel names and pseudoword filles. 


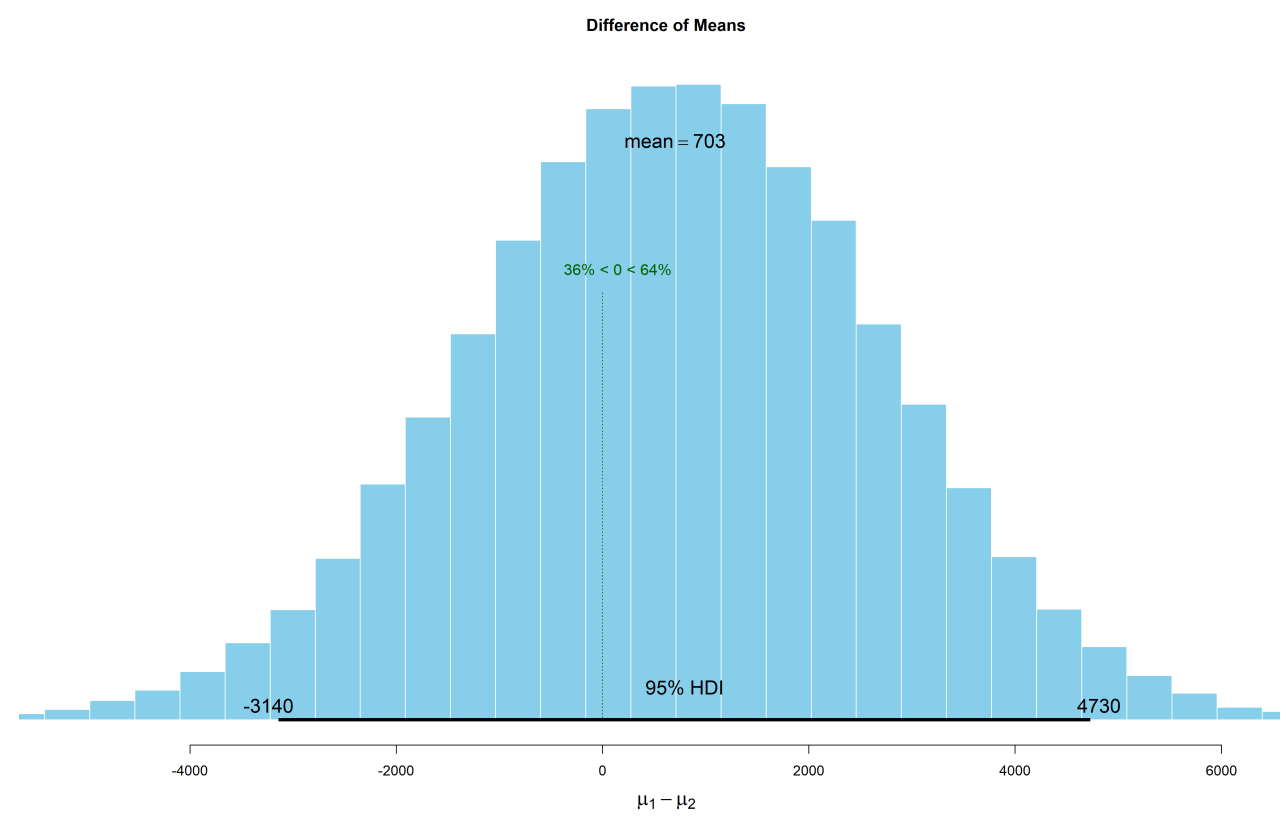

Figure 41. Posterior distribution of the difference in means for unconstrained bigram frequency for the trained novel names and pseudoword filles.

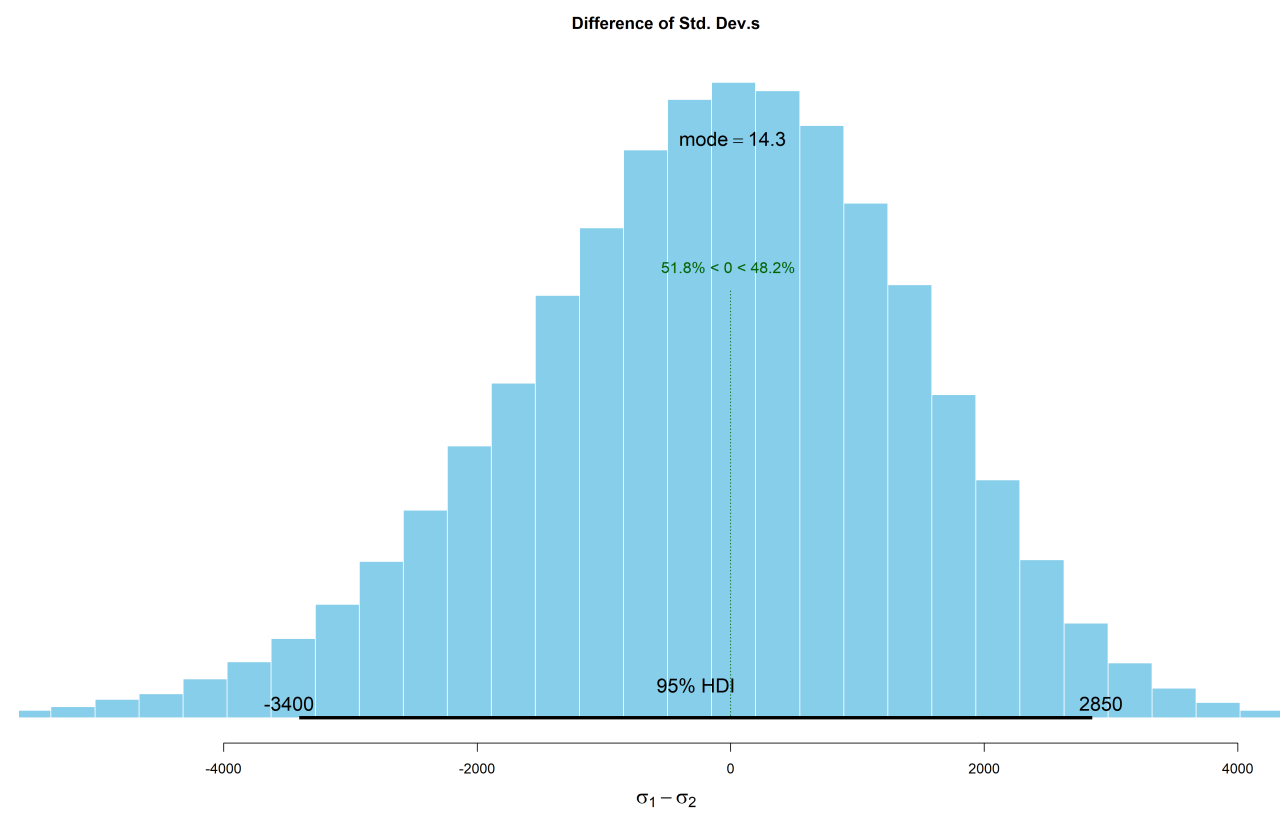

Figure 42. Posterior distribution of the difference in standard deviation for unconstrained bigram frequency for the trained novel names and pseudoword filles. 


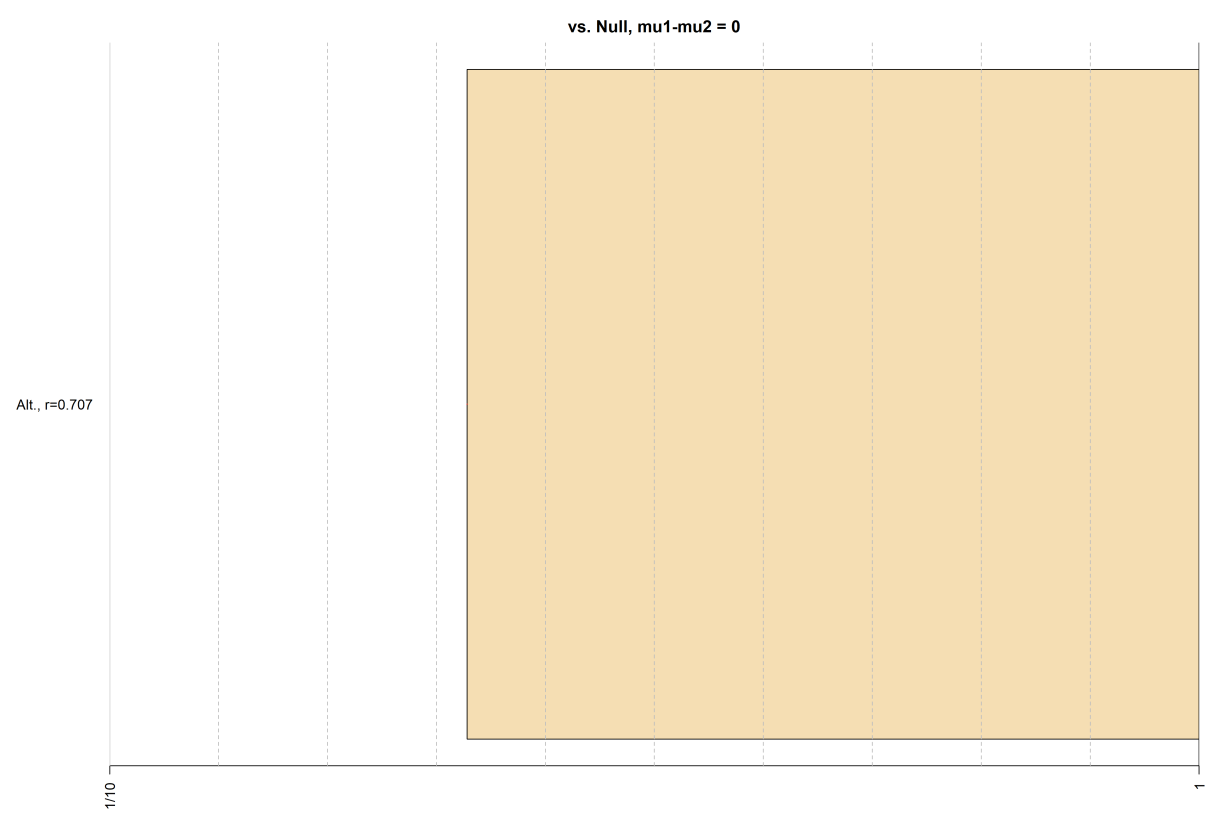

Figure 43. Graphical representation of the Bayes factor (denominator model: $\mu_{1}-\mu_{2}=$ $0)$.
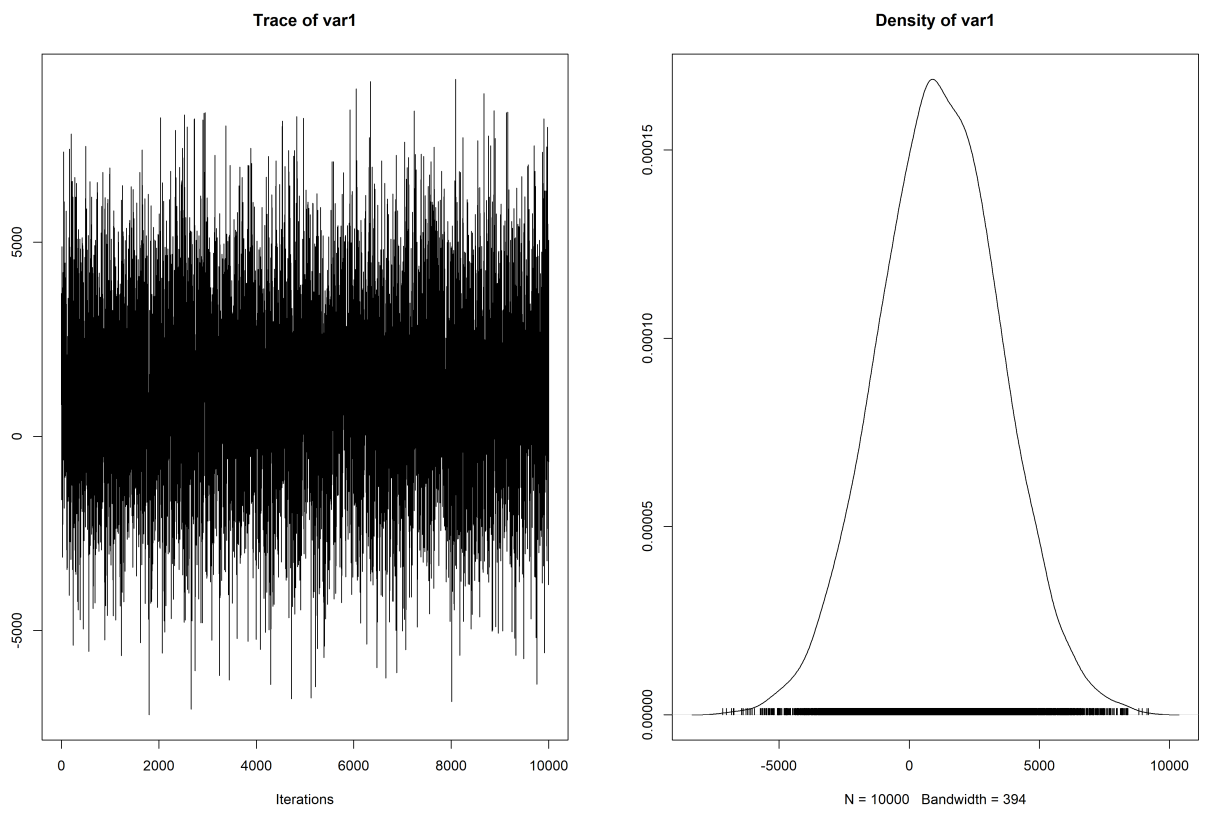

Figure 44. Sampling diagnostics of the posterior for the numerator model. 
Constrained bigram frequency for the trained novel names and pseudoword filles

\begin{tabular}{lccccccc}
\hline & Mean & Median & Mode & $\begin{array}{c}95 \% \text { HDI } \\
\text { lower }\end{array}$ & $\begin{array}{c}95 \% \text { HDI } \\
\text { upper }\end{array}$ & $\begin{array}{c}\text { Comp. } \\
\text { value }\end{array}$ & $\begin{array}{r}\%> \\
\text { Comp. } \\
\text { value }\end{array}$ \\
\hline$\mu_{1}$ & 583.994 & 583.342 & 583.309 & 508.196 & 657.999 & & \\
$\mu_{2}$ & 529.153 & 527.262 & 523.303 & 402.035 & 661.402 & & \\
$\mu_{\text {diff }}$ & 54.841 & 56.045 & 58.257 & -95.546 & 201.191 & 0 & 77.300 \\
$\sigma_{1}$ & 342.301 & 339.835 & 340.510 & 265.637 & 424.583 & & \\
$\sigma_{2}$ & 314.517 & 306.128 & 289.980 & 184.230 & 466.172 & & \\
$\sigma_{d i f f}$ & 27.784 & 33.938 & 48.628 & -131.012 & 177.987 & 0 & 66.600 \\
$\nu$ & 1.267 & 1.246 & 1.209 & 1.000 & 1.568 & & \\
$\log _{10} \nu$ & 0.099 & 0.0955 & 0.086 & 0.000 & 0.195 & & \\
effect size & 0.170 & 0.172 & 0.182 & -0.289 & 0.611 & 0 & 77.300 \\
\hline
\end{tabular}

Table 19. Bayesian estimation for constrained bigram frequency for the trained novel names and pseudoword filles.

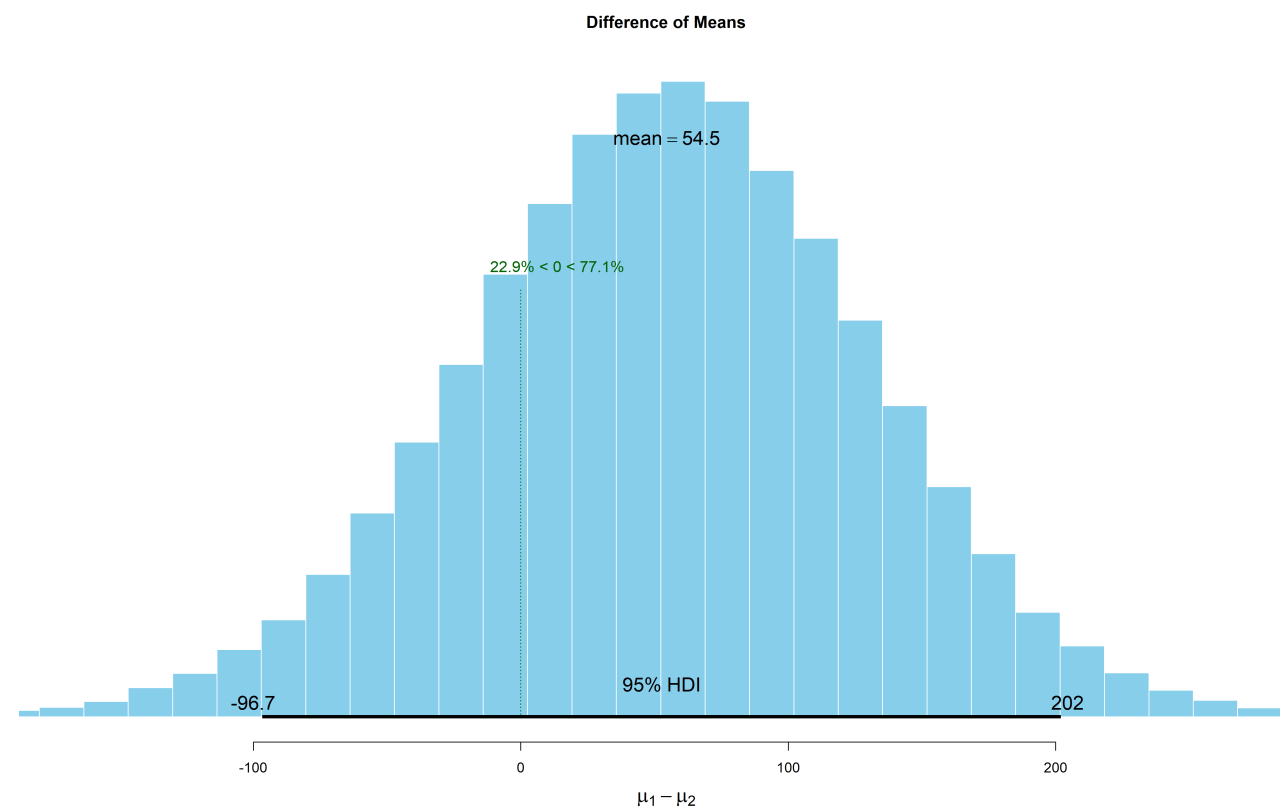

Figure 45. Posterior distribution of the difference in means for constrained bigram frequency for the trained novel names and pseudoword filles. 


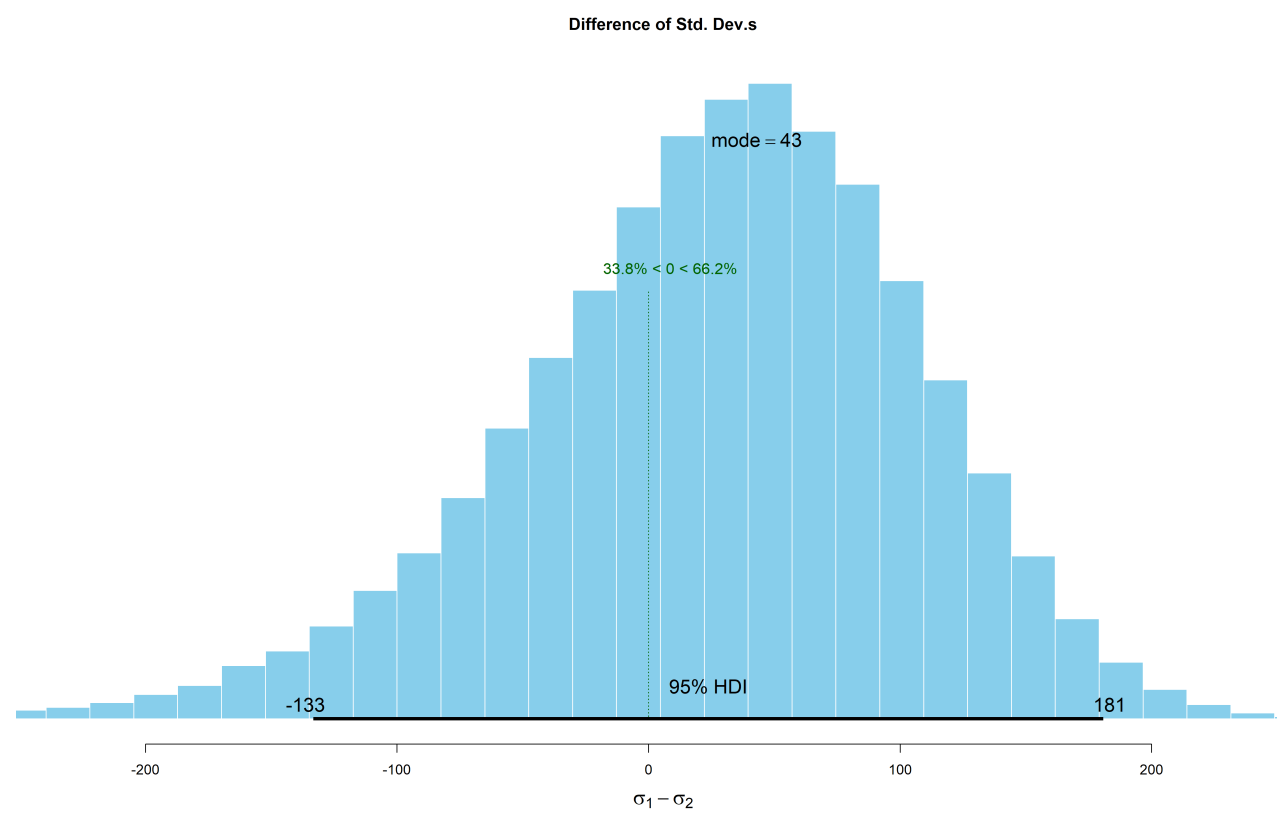

Figure 46. Posterior distribution of the difference in standard deviation for constrained bigram frequency for the trained novel names and pseudoword filles.

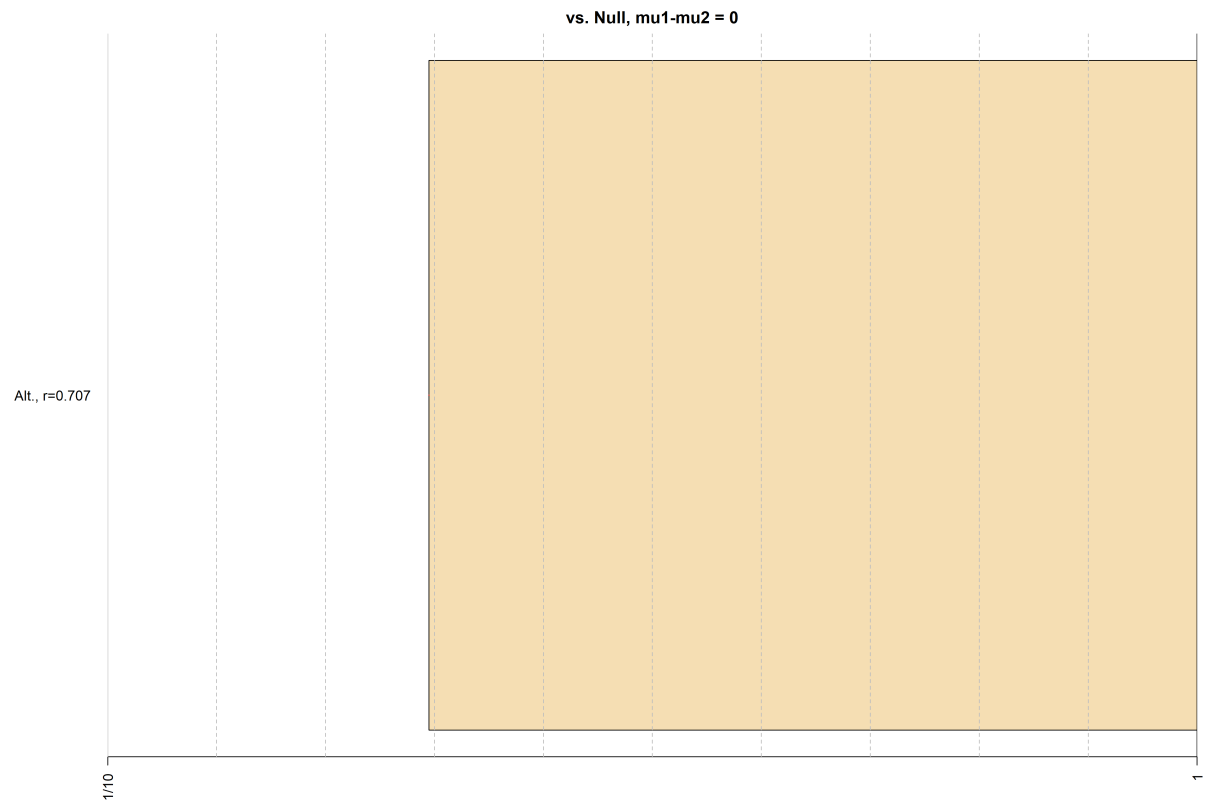

Figure 47. Graphical representation of the Bayes factor (denominator model: $\mu_{1}-\mu_{2}=$ $0)$. 

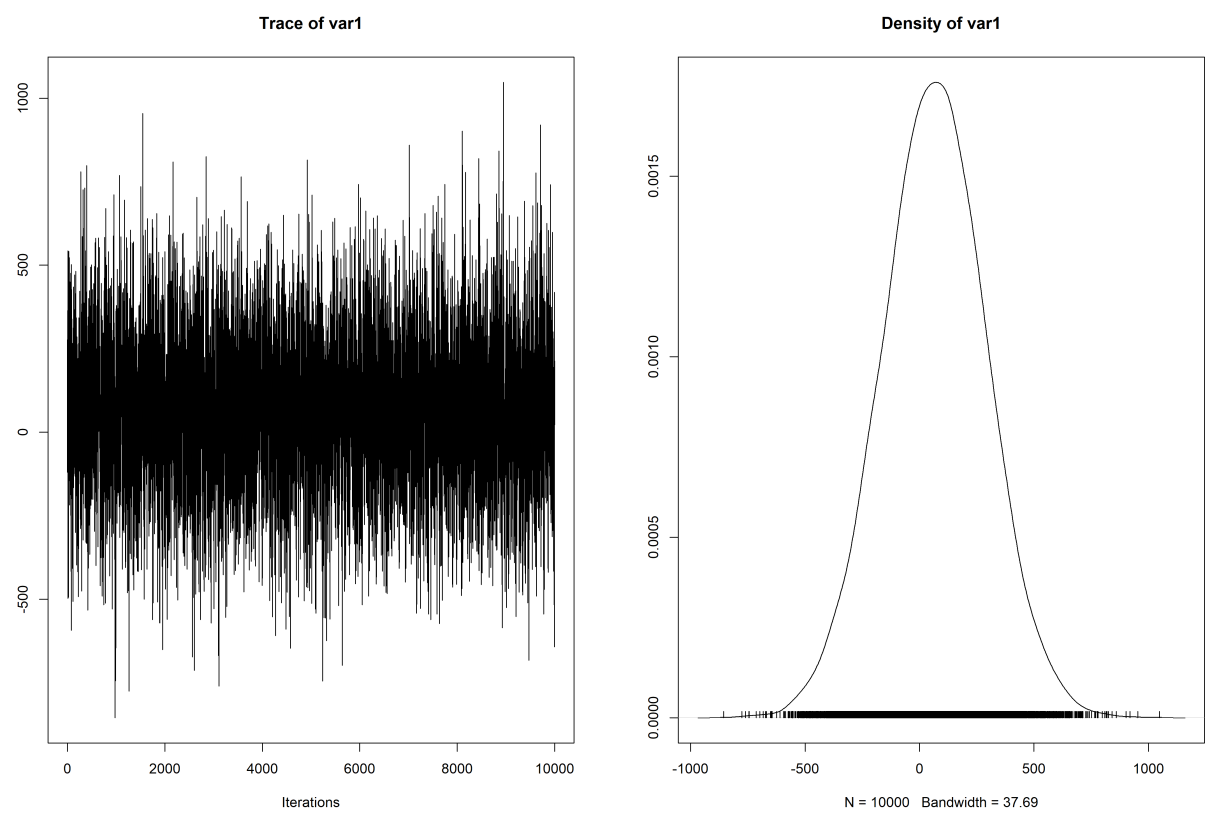

Figure 48. Sampling diagnostics of the posterior for the numerator model.

OLD20 for the English targets preceded by semantically related and unrelated primes

\begin{tabular}{lccccccc}
\hline & Mean & Median & Mode & $\begin{array}{c}95 \% \text { HDI } \\
\text { lower }\end{array}$ & $\begin{array}{c}95 \% \text { HDI } \\
\text { upper }\end{array}$ & $\begin{array}{c}\text { Comp. } \\
\text { value }\end{array}$ & $\begin{array}{r}\%> \\
\text { Comp. } \\
\text { value }\end{array}$ \\
\hline$\mu_{1}$ & 2.404 & 2.403 & 2.391 & 2.034 & 2.757 & & \\
$\mu_{2}$ & 2.814 & 2.813 & 2.804 & 2.417 & 3.211 & & \\
$\mu_{\text {diff }}$ & -0.411 & -0.410 & -0.397 & -0.948 & 0.122 & 0 & 6.420 \\
$\sigma_{1}$ & 0.770 & 0.752 & 0.701 & 0.508 & 1.070 & & \\
$\sigma_{2}$ & 0.849 & 0.829 & 0.782 & 0.571 & 1.171 & & \\
$\sigma_{\text {diff }}$ & -0.079 & -0.076 & -0.067 & -0.502 & 0.353 & 0 & 35.070 \\
$\nu$ & 36.562 & 28.059 & 13.108 & 1.920 & 95.544 & & \\
$\log _{10} \nu$ & 1.427 & 1.448 & 1.492 & 0.721 & 2.088 & & \\
effect size & -0.512 & -0.510 & -0.518 & -1.188 & 0.140 & 0 & 6.420 \\
\hline
\end{tabular}

Table 20. Bayesian estimation for OLD20 for the English targets preceded by semantically related and unrelated primes. 


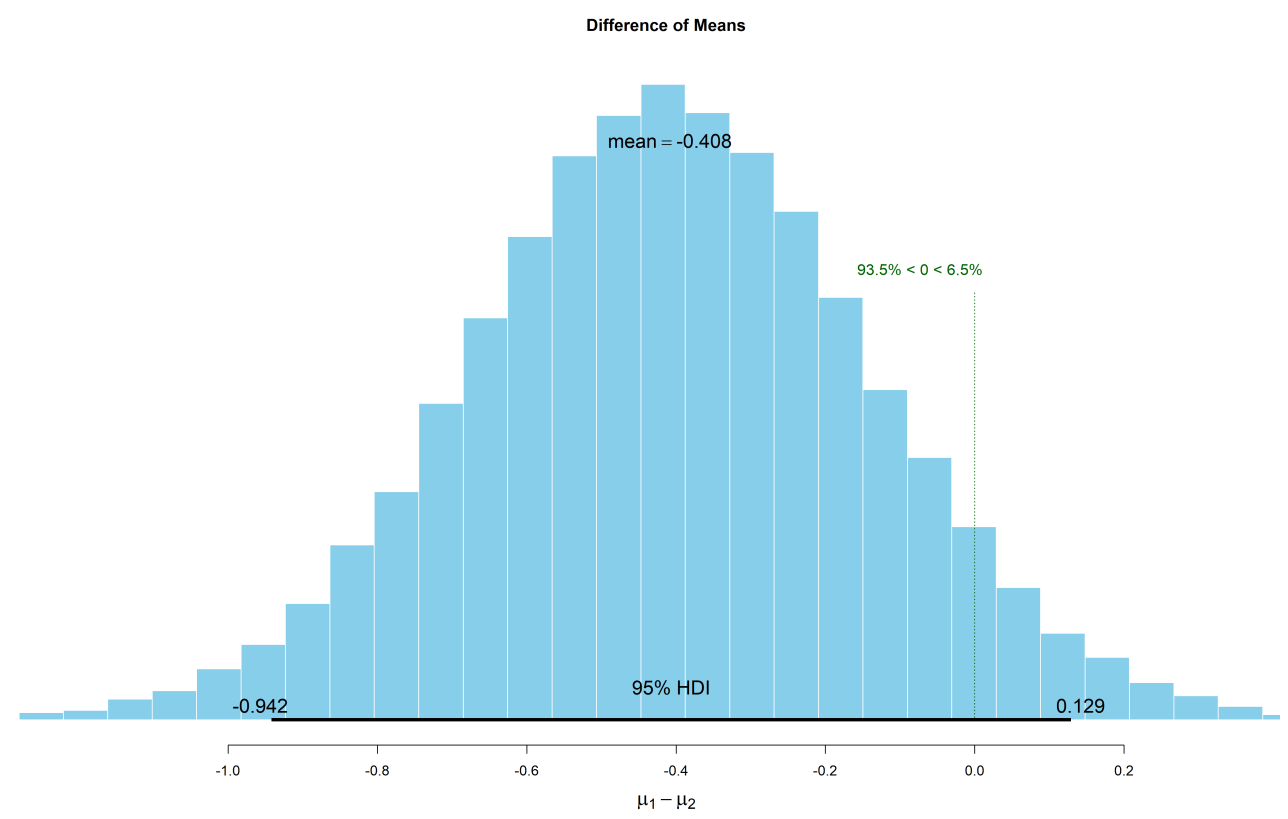

Figure 49. Posterior distribution of the difference in means for OLD20 for the English targets preceded by semantically related and unrelated primes.

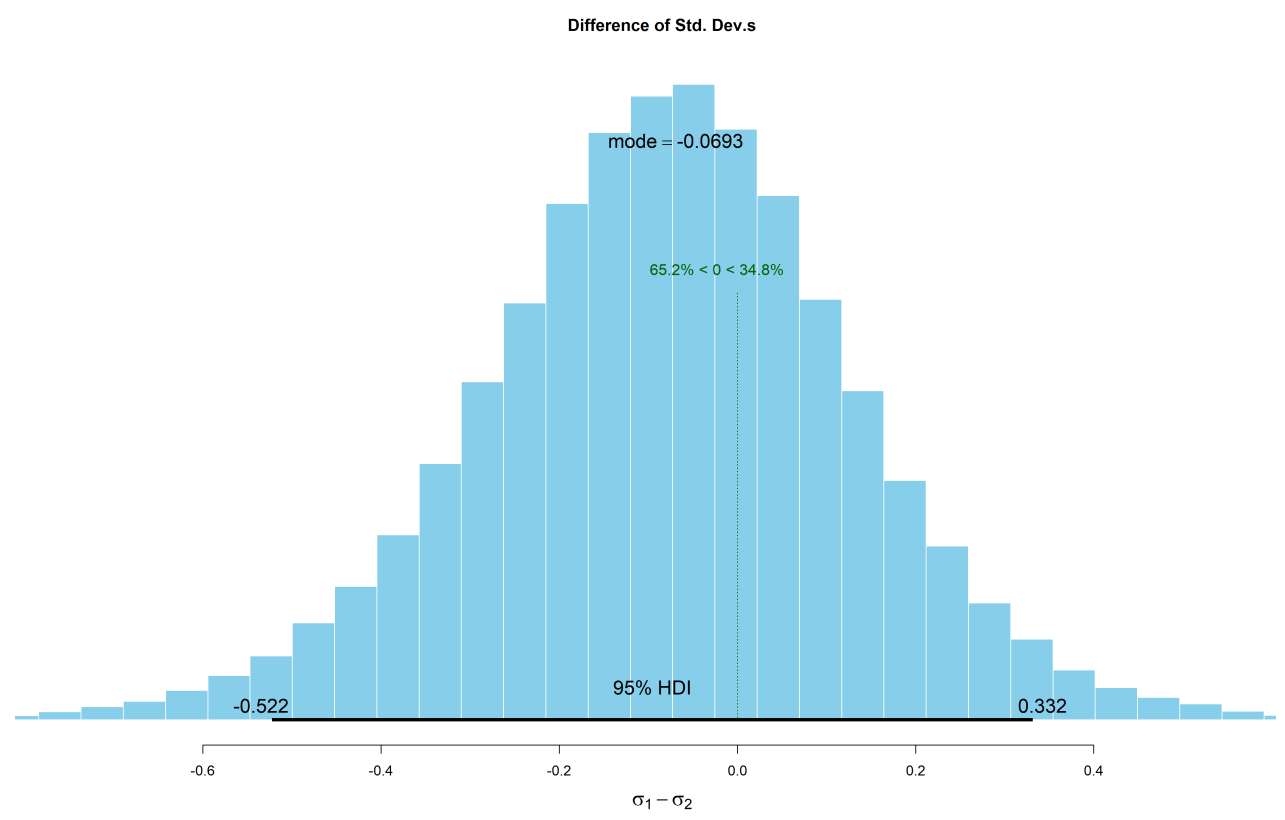

Figure 50. Posterior distribution of the difference in standard deviation for OLD20 for the English targets preceded by semantically related and unrelated primes. 


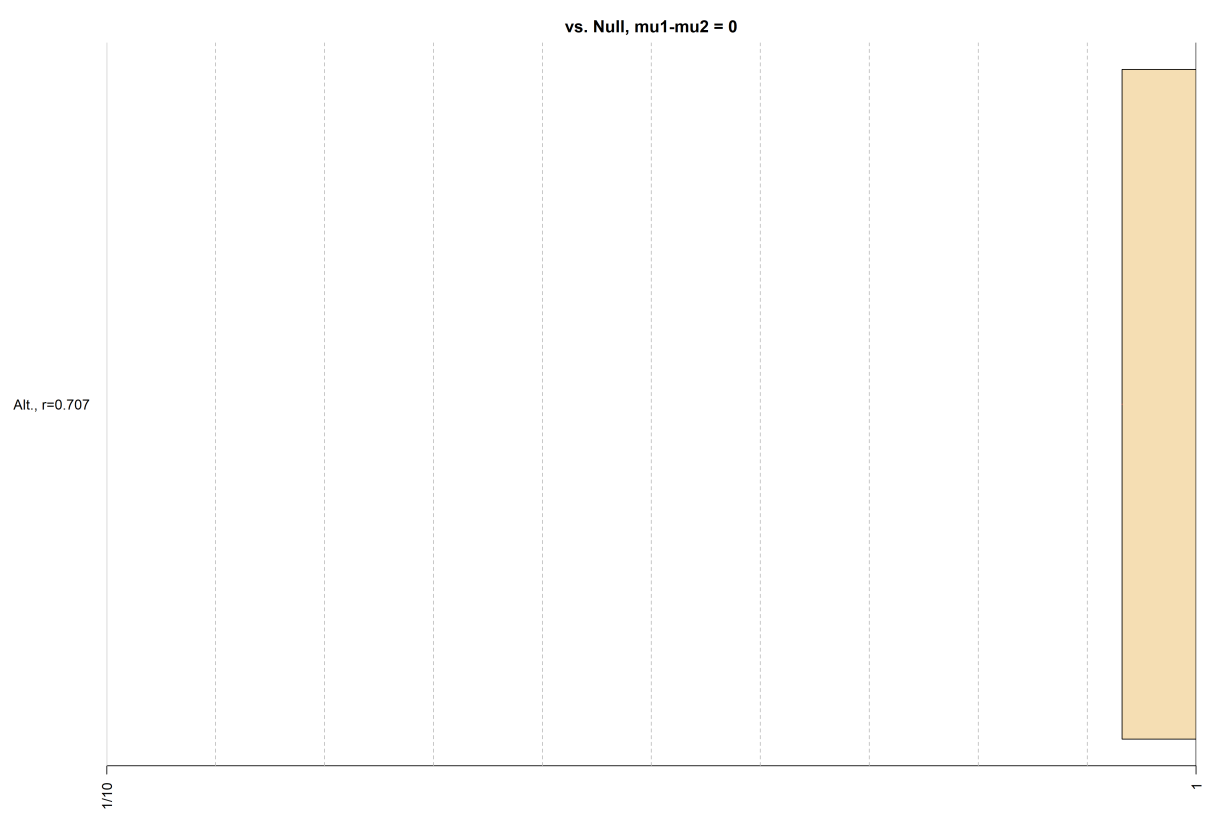

Figure 51. Graphical representation of the Bayes factor (denominator model: $\mu_{1}-\mu_{2}=$ $0)$.
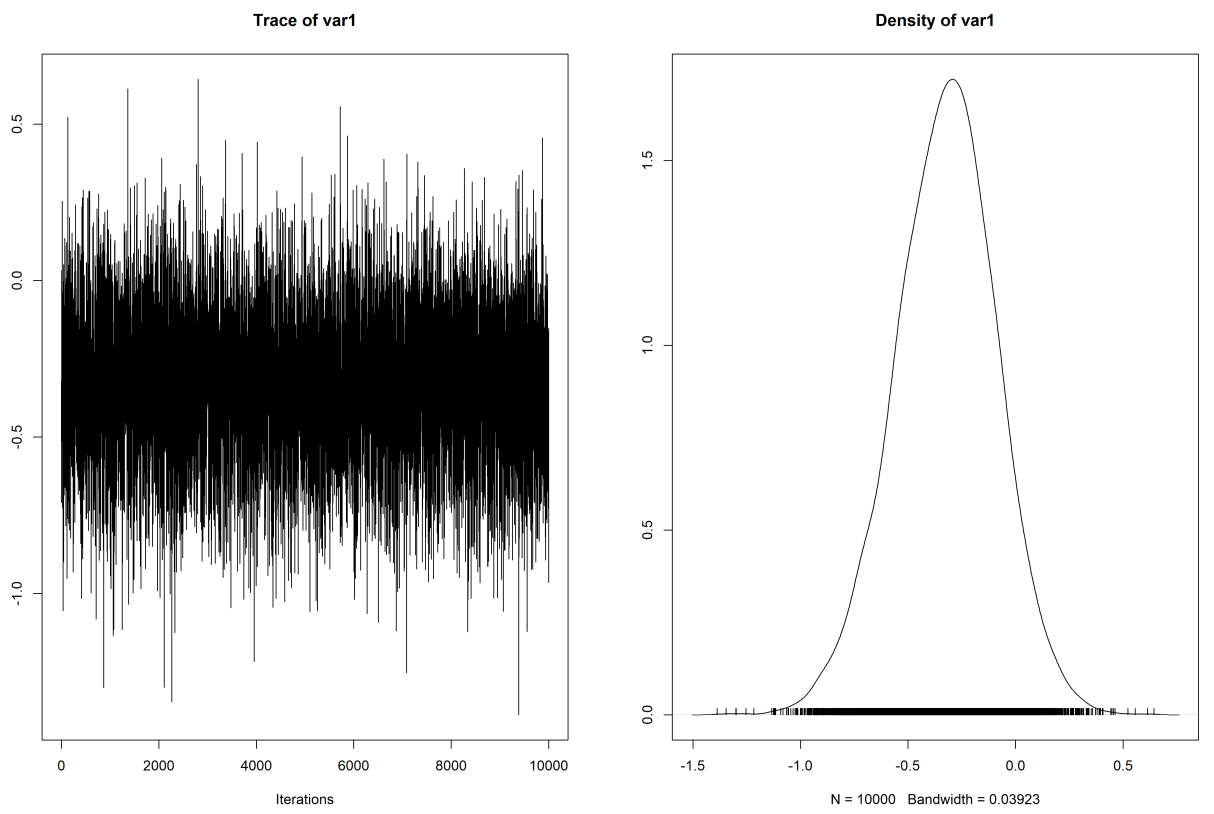

Figure 52. Sampling diagnostics of the posterior for the numerator model. 
Number of letters for the English targets preceded by semantically related and unrelated primes

\begin{tabular}{lccccccc}
\hline & Mean & Median & Mode & $\begin{array}{c}95 \% \text { HDI } \\
\text { lower }\end{array}$ & $\begin{array}{c}95 \% \text { HDI } \\
\text { upper }\end{array}$ & $\begin{array}{c}\text { Comp. } \\
\text { value }\end{array}$ & $\begin{array}{r}\%> \\
\text { Comp. } \\
\text { value }\end{array}$ \\
\hline$\mu_{1}$ & 5.866 & 5.862 & 5.843 & 4.934 & 6.7837 & & \\
$\mu_{2}$ & 6.929 & 6.928 & 6.938 & 6.089 & 7.781 & & \\
$\mu_{\text {diff }}$ & -1.063 & -1.067 & -1.113 & -2.318 & 0.179 & 0 & 4.690 \\
$\sigma_{1}$ & 1.978 & 1.933 & 1.845 & 1.316 & 2.750 & & \\
$\sigma_{2}$ & 1.807 & 1.764 & 1.703 & 1.226 & 2.494 & & \\
$\sigma_{\text {diff }}$ & 0.170 & 0.166 & 0.161 & -0.815 & 1.172 & 0 & 64.030 \\
$\nu$ & 38.030 & 29.481 & 13.277 & 2.149 & 98.513 & & \\
$\log _{10} \nu$ & 1.447 & 1.470 & 1.482 & 0.750 & 2.106 & & 4.690 \\
effect size & -0.567 & -0.567 & -0.557 & -1.230 & 0.0980 & 0 & \\
\hline
\end{tabular}

Table 21. Bayesian estimation for number of letters for the English targets preceded by semantically related and unrelated primes.

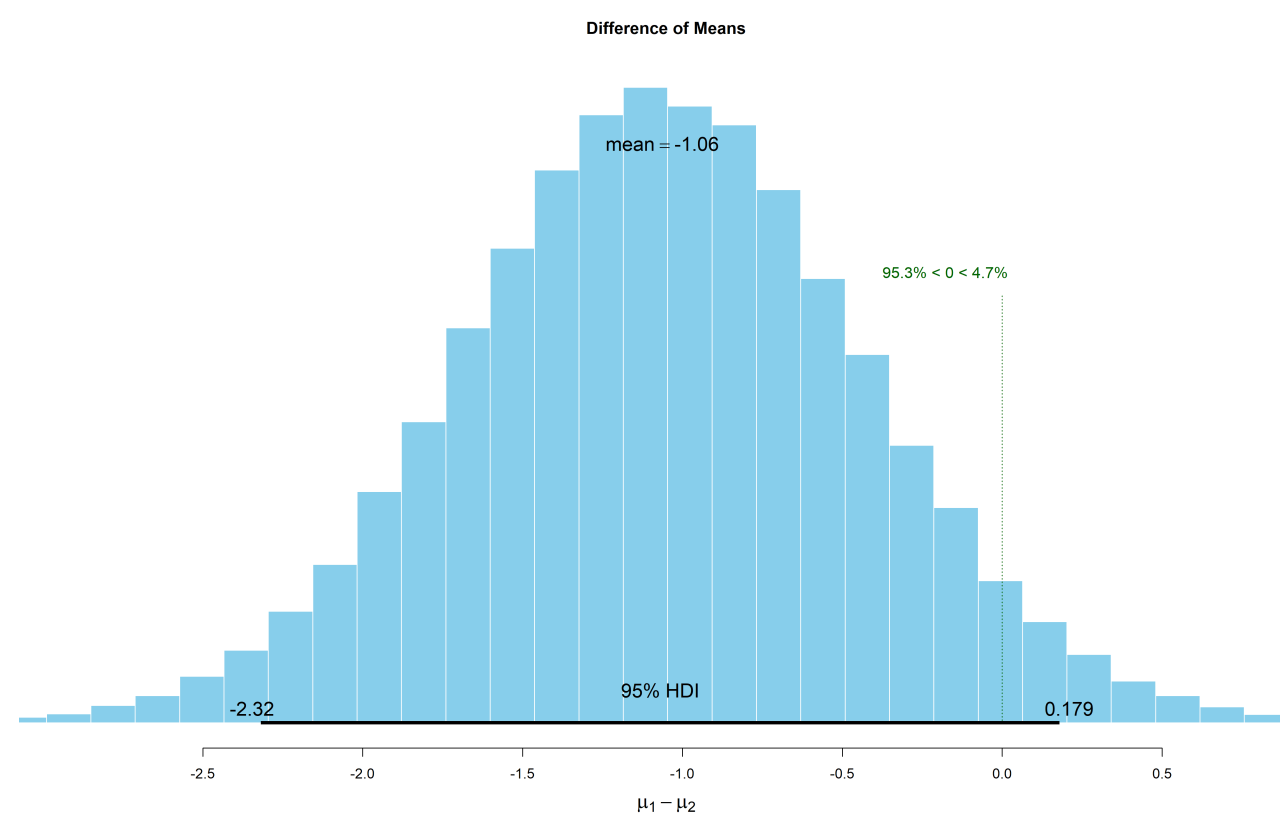

Figure 53. Posterior distribution of the difference in means for number of letters for the English targets preceded by semantically related and unrelated primes. 


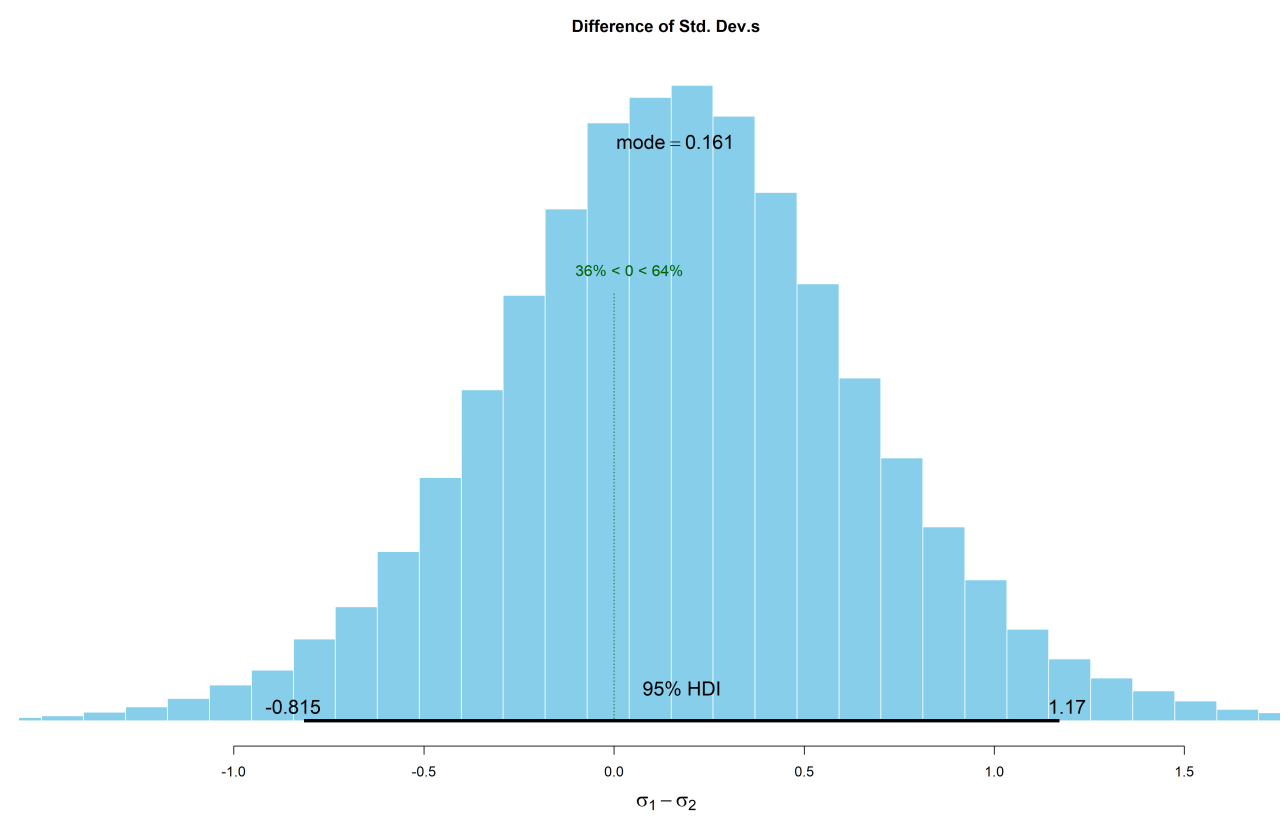

Figure 54. Posterior distribution of the difference in standard deviation for number of letters for the English targets preceded by semantically related and unrelated primes.

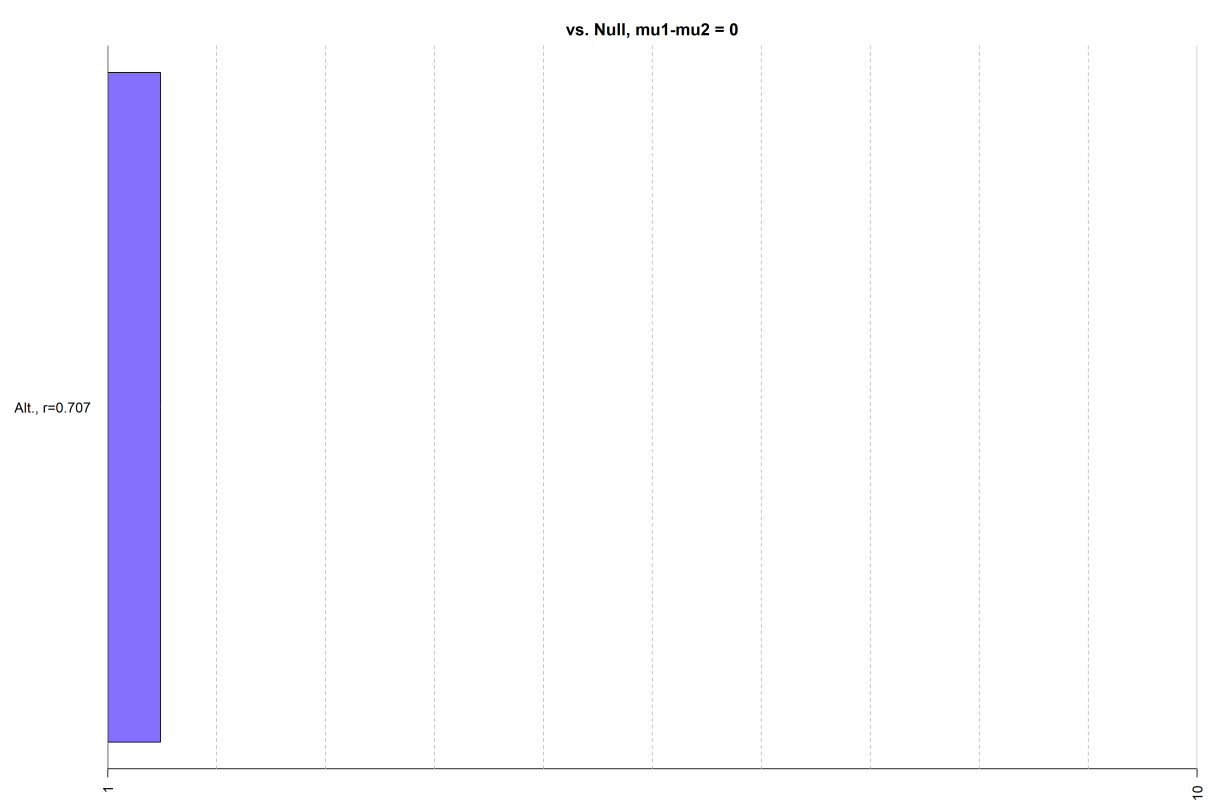

Figure 55. Graphical representation of the Bayes factor (denominator model: $\mu_{1}-\mu_{2}=$ $0)$. 

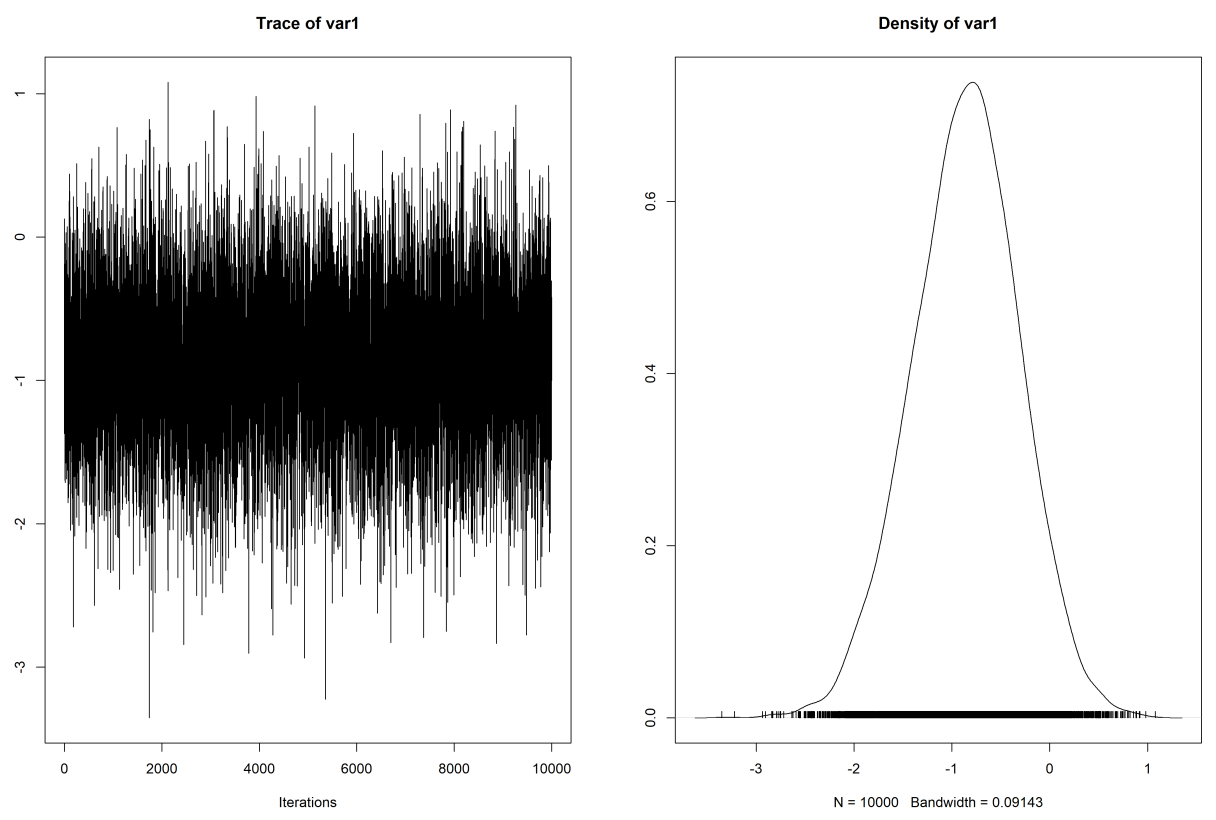

Figure 56. Sampling diagnostics of the posterior for the numerator model.

Word frequency for the English targets preceded by semantically related and unrelated primes

\begin{tabular}{lccccccr}
\hline & Mean & Median & Mode & $\begin{array}{c}95 \% \text { HDI } \\
\text { lower }\end{array}$ & $\begin{array}{c}95 \% \text { HDI } \\
\text { upper }\end{array}$ & $\begin{array}{c}\text { Comp. } \\
\text { value }\end{array}$ & $\begin{array}{r}\%> \\
\text { Comp. } \\
\text { value }\end{array}$ \\
\hline$\mu_{1}$ & 4.581 & 4.083 & 3.125 & 0.889 & 9.674 & & \\
$\mu_{2}$ & 2.556 & 2.540 & 2.560 & 1.347 & 3.806 & & \\
$\mu_{\text {diff }}$ & 2.025 & 1.570 & 0.857 & -1.916 & 7.242 & 0 & 81.100 \\
$\sigma_{1}$ & 6.338 & 5.869 & 4.929 & 1.609 & 12.015 & & \\
$\sigma_{2}$ & 1.935 & 1.820 & 1.685 & 0.807 & 3.309 & & \\
$\sigma_{d i f f}$ & 4.403 & 3.979 & 3.168 & -0.420 & 10.115 & 0 & 97.900 \\
$\nu$ & 1.505 & 1.357 & 1.079 & 1.000 & 2.470 & & \\
$\log _{10} \nu$ & 0.158 & 0.133 & 0.028 & 0.000 & 0.393 & & \\
effect size & 0.373 & 0.367 & 0.334 & -0.474 & 1.228 & 0 & 81.100 \\
\hline
\end{tabular}

Table 22. Bayesian estimation for word frequency for the English targets preceded by semantically related and unrelated primes. 


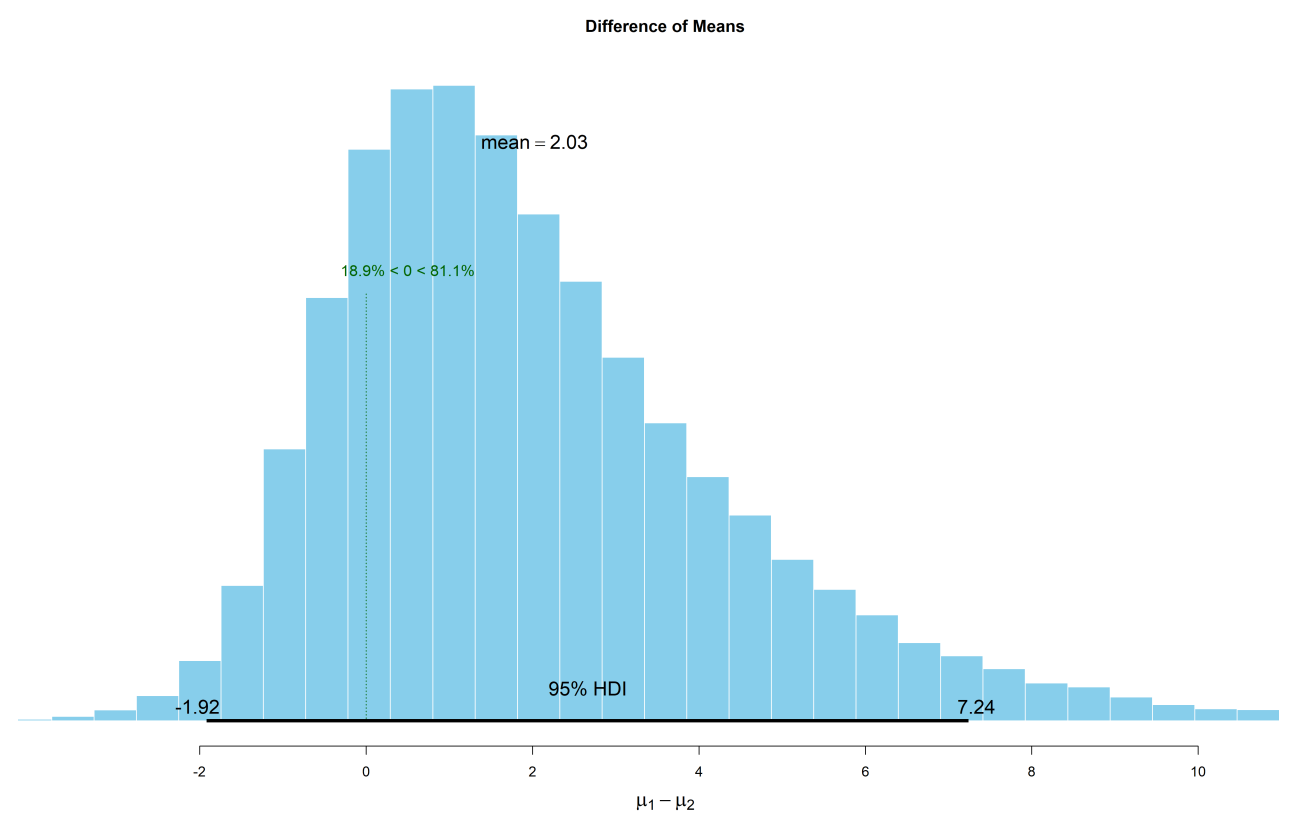

Figure 57. Posterior distribution of the difference in means for word frequency for the English targets preceded by semantically related and unrelated primes.

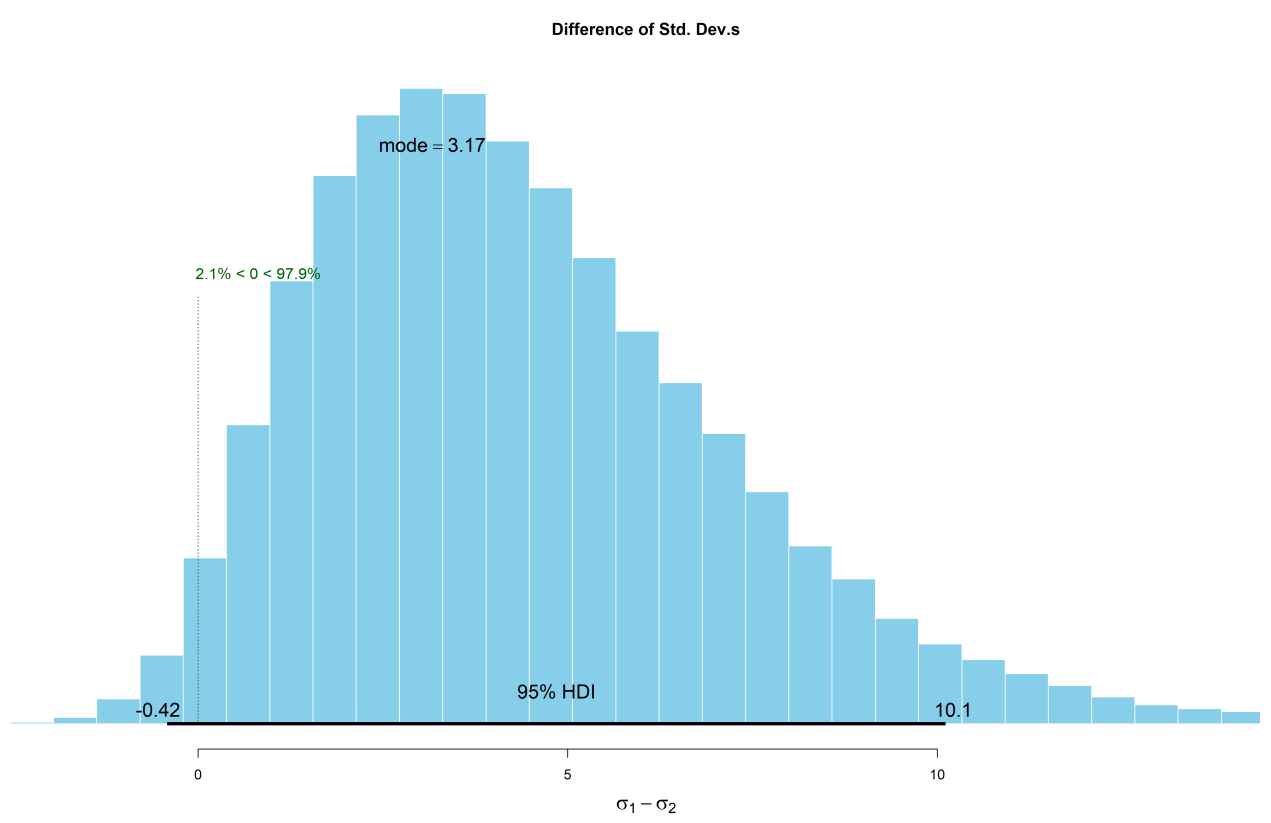

Figure 58. Posterior distribution of the difference in standard deviation for word frequency for the English targets preceded by semantically related and unrelated primes. 


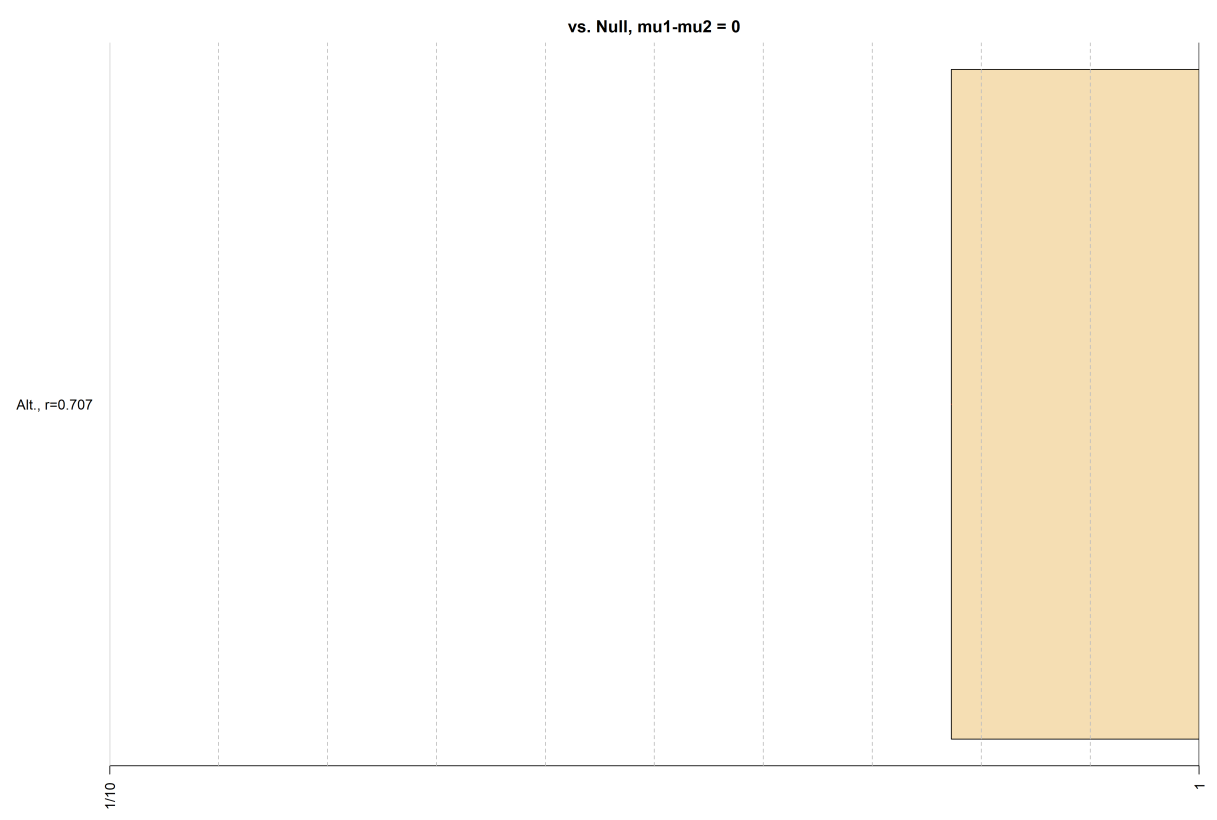

Figure 59. Graphical representation of the Bayes factor (denominator model: $\mu_{1}-\mu_{2}=$ $0)$.
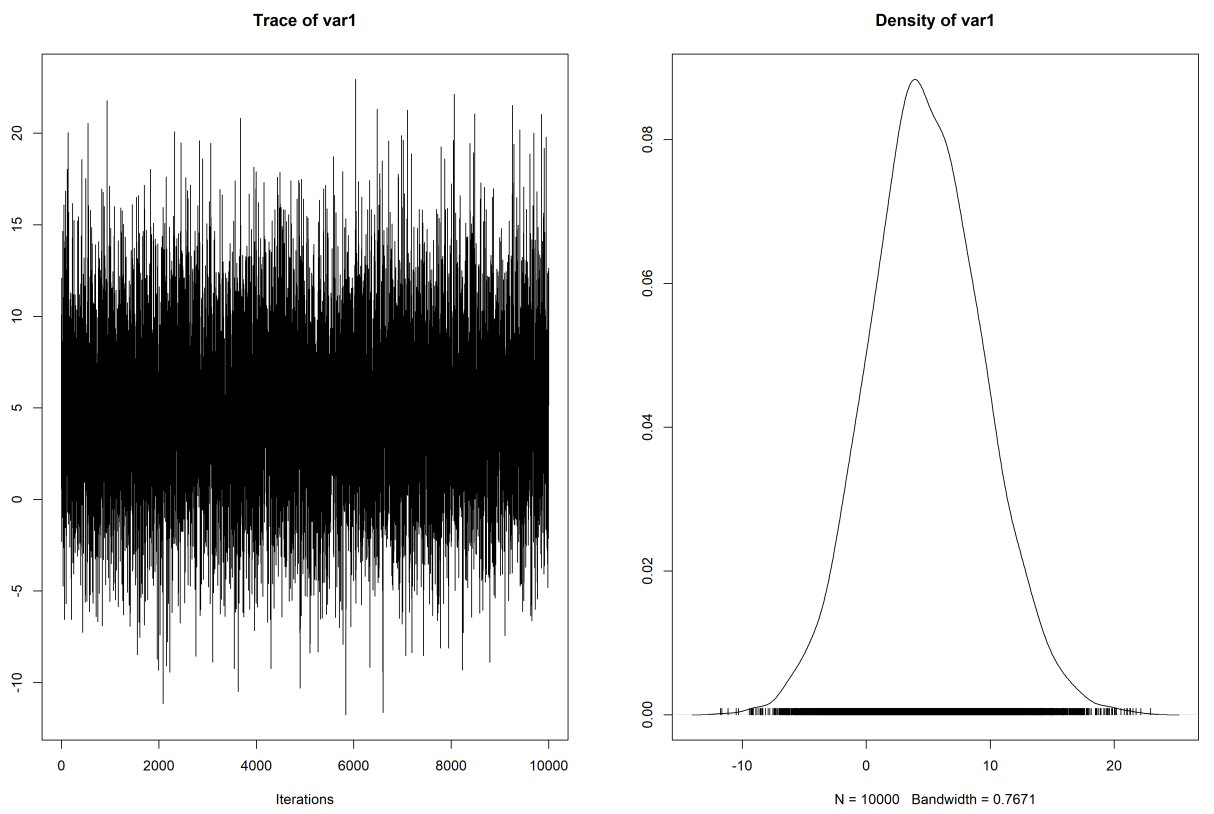

Figure 60. Sampling diagnostics of the posterior for the numerator model. 
OLD20 for the trained novel name targets preceded by semantically related and unrelated primes

\begin{tabular}{lccccccc}
\hline & Mean & Median & Mode & $\begin{array}{c}95 \% \text { HDI } \\
\text { lower }\end{array}$ & $\begin{array}{c}95 \% \text { HDI } \\
\text { upper }\end{array}$ & $\begin{array}{c}\text { Comp. } \\
\text { value }\end{array}$ & $\begin{array}{r}\%> \\
\text { Comp. } \\
\text { value }\end{array}$ \\
\hline$\mu_{1}$ & 2.111 & 2.111 & 2.116 & 1.881 & 2.340 & & \\
$\mu_{2}$ & 2.052 & 2.051 & 2.049 & 1.764 & 2.334 & & \\
$\mu_{\text {diff }}$ & 0.059 & 0.060 & 0.074 & -0.308 & 0.418 & 0 & 63.000 \\
$\sigma_{1}$ & 0.490 & 0.478 & 0.460 & 0.320 & 0.678 & & \\
$\sigma_{2}$ & 0.607 & 0.593 & 0.579 & 0.401 & 0.847 & & \\
$\sigma_{\text {diff }}$ & -0.117 & -0.113 & -0.092 & -0.413 & 0.172 & 0 & 20.100 \\
$\nu$ & 36.683 & 28.084 & 13.059 & 1.639 & 96.535 & & \\
$\log _{10} \nu$ & 1.425 & 1.449 & 1.528 & 0.704 & 2.101 & & \\
effect size & 0.110 & 0.110 & 0.130 & -0.545 & 0.764 & 0 & 63.000 \\
\hline
\end{tabular}

Table 23. Bayesian estimation for OLD20 for the trained novel name targets preceded by semantically related and unrelated primes.

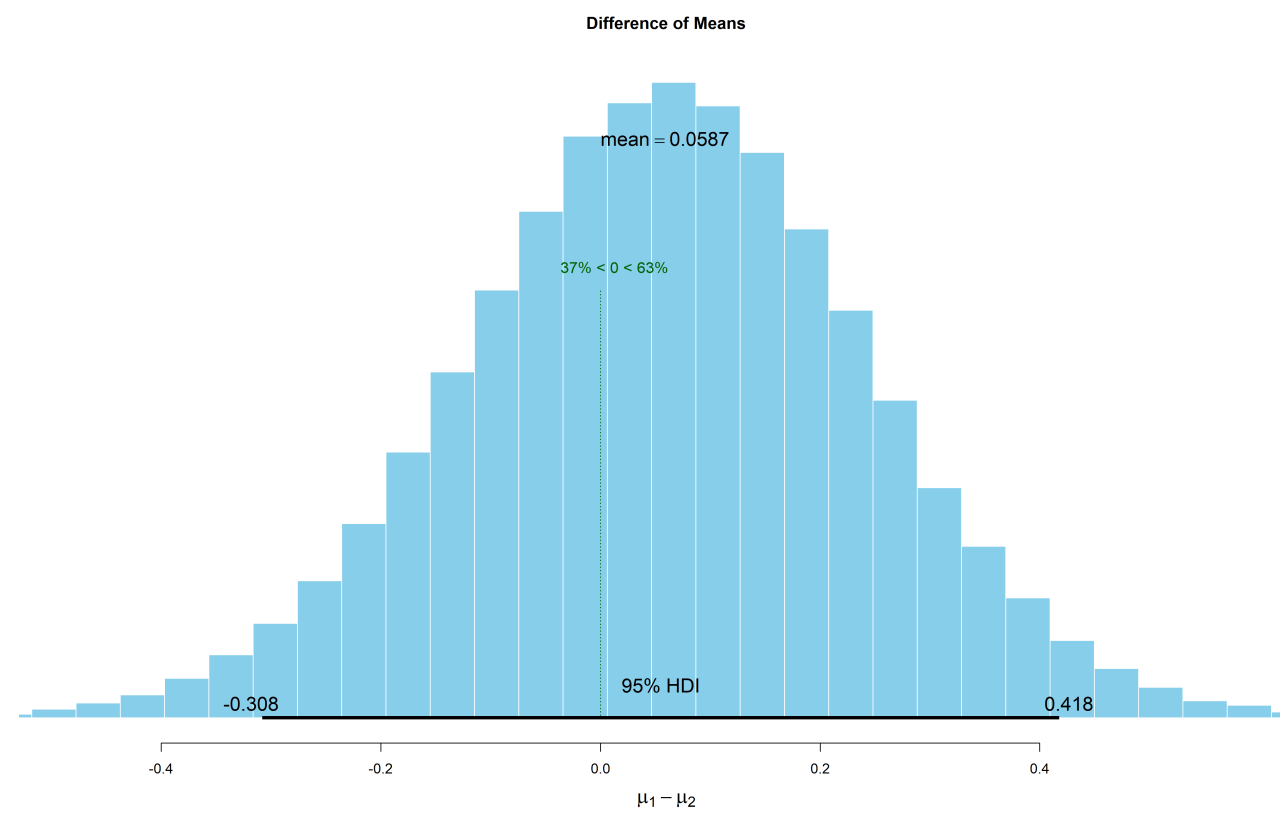

Figure 61. Posterior distribution of the difference in means for OLD20 for the trained novel name targets preceded by semantically related and unrelated primes. 


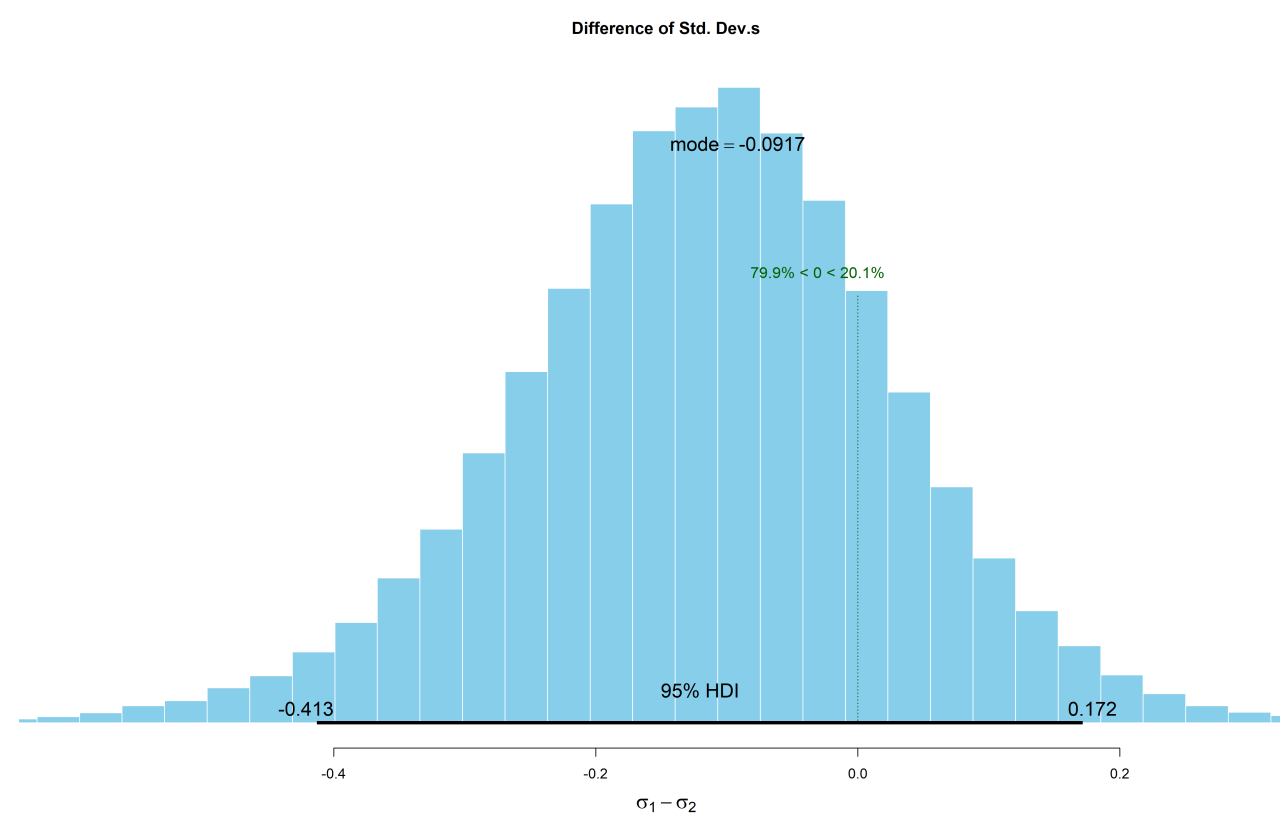

Figure 62. Posterior distribution of the difference in standard deviation for OLD20 for the trained novel name targets preceded by semantically related and unrelated primes.

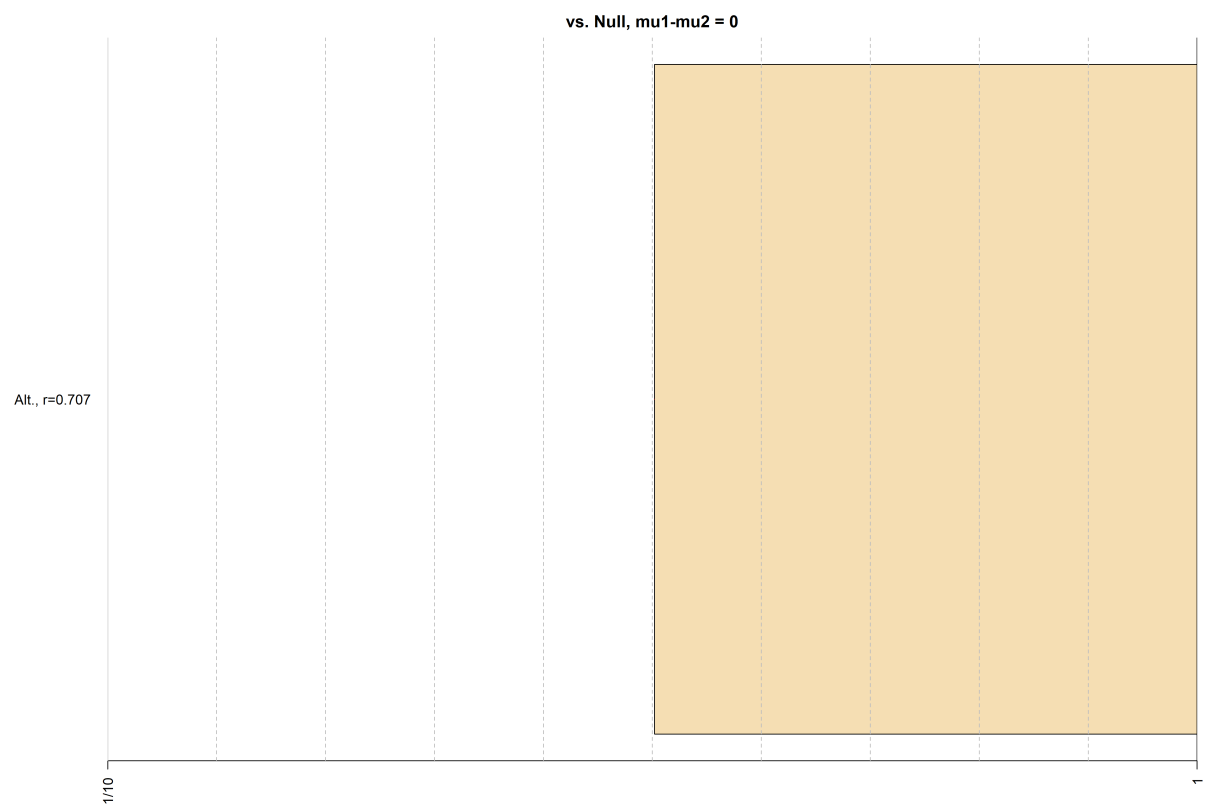

Figure 63. Graphical representation of the Bayes factor (denominator model: $\mu_{1}-\mu_{2}=$ $0)$. 

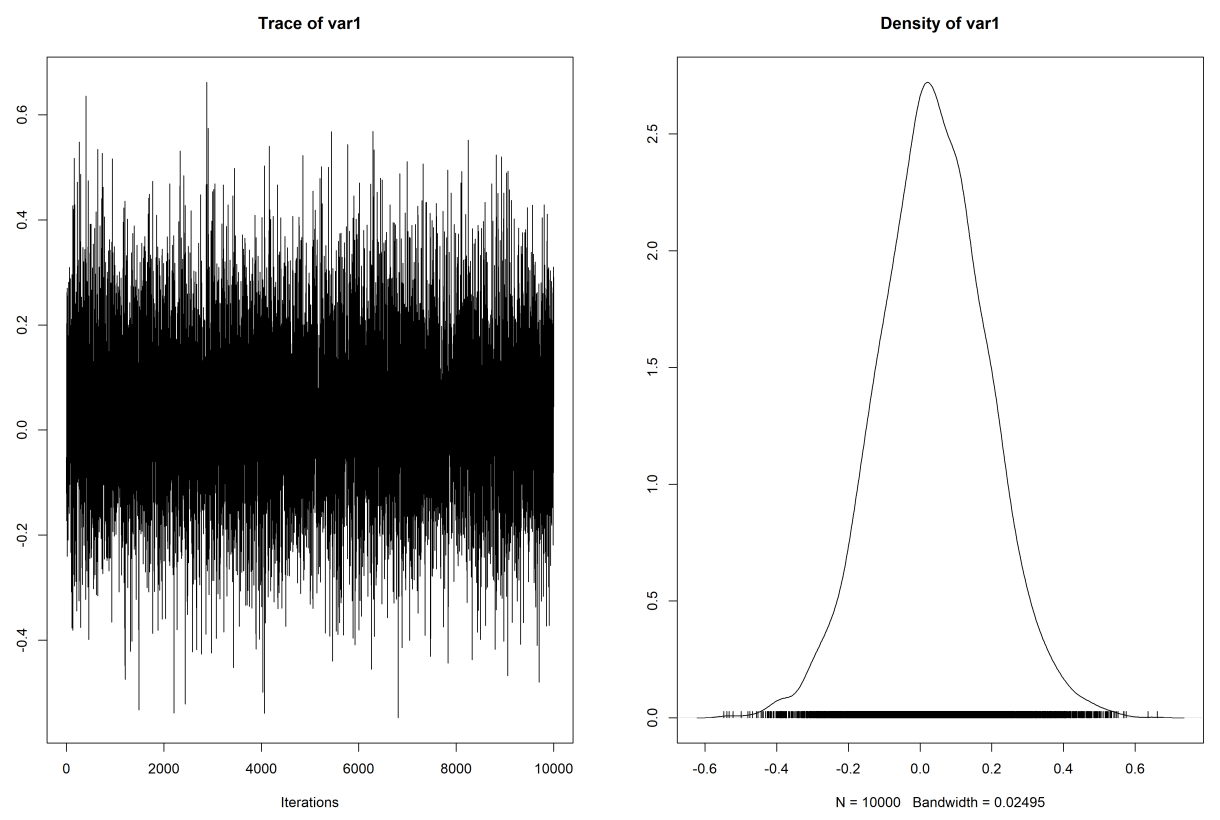

Figure 64. Sampling diagnostics of the posterior for the numerator model.

Number of letters for the trained novel name targets preceded by semantically related and unrelated primes

\begin{tabular}{lccccccc}
\hline & Mean & Median & Mode & $\begin{array}{c}95 \% \text { HDI } \\
\text { lower }\end{array}$ & $\begin{array}{c}95 \% \text { HDI } \\
\text { upper }\end{array}$ & $\begin{array}{c}\text { Comp. } \\
\text { value }\end{array}$ & $\begin{array}{r}\%> \\
\text { Comp. } \\
\text { value }\end{array}$ \\
\hline$\mu_{1}$ & 5.412 & 5.413 & 5.437 & 4.832 & 5.982 & & \\
$\mu_{2}$ & 5.296 & 5.295 & 5.275 & 4.693 & 5.899 & & \\
$\mu_{\text {diff }}$ & 0.117 & 0.117 & 0.131 & -0.714 & 0.940 & 0 & 61.100 \\
$\sigma_{1}$ & 1.240 & 1.211 & 1.177 & 0.831 & 1.706 & & \\
$\sigma_{2}$ & 1.291 & 1.2608 & 1.203 & 0.834 & 1.781 & & \\
$\sigma_{d i f f}$ & -0.051 & -0.049 & -0.044 & -0.726 & 0.613 & 0 & 43.800 \\
$\nu$ & 36.678 & 28.217 & 13.348 & 2.103 & 96.413 & & \\
$\log _{10} \nu$ & 1.431 & 1.451 & 1.509 & 0.713 & 2.073 & & \\
effect size & 0.093 & 0.093 & 0.096 & -0.533 & 0.752 & 0 & 61.100 \\
\hline
\end{tabular}

Table 24. Bayesian estimation for number of letters for the trained novel name targets preceded by semantically related and unrelated primes. 


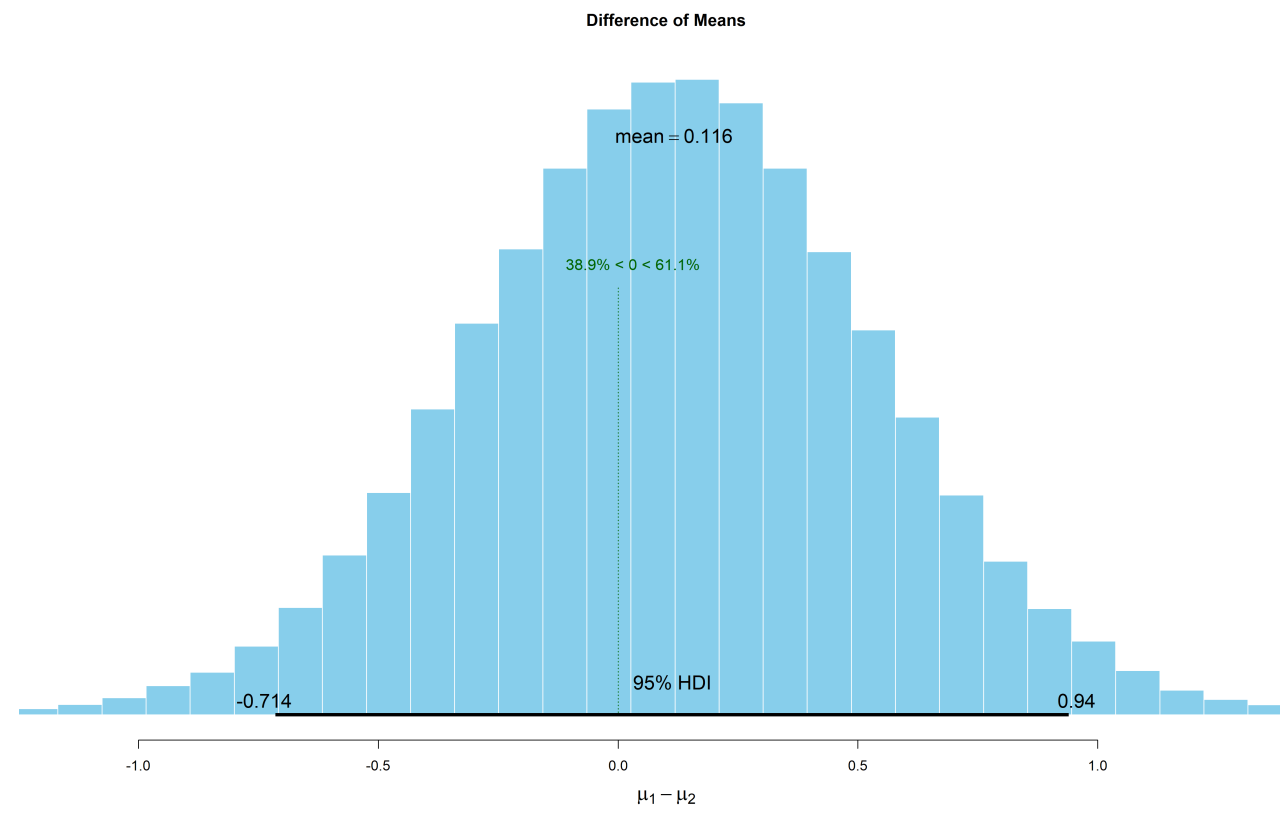

Figure 65. Posterior distribution of the difference in means for number of letters for the trained novel name targets preceded by semantically related and unrelated primes.

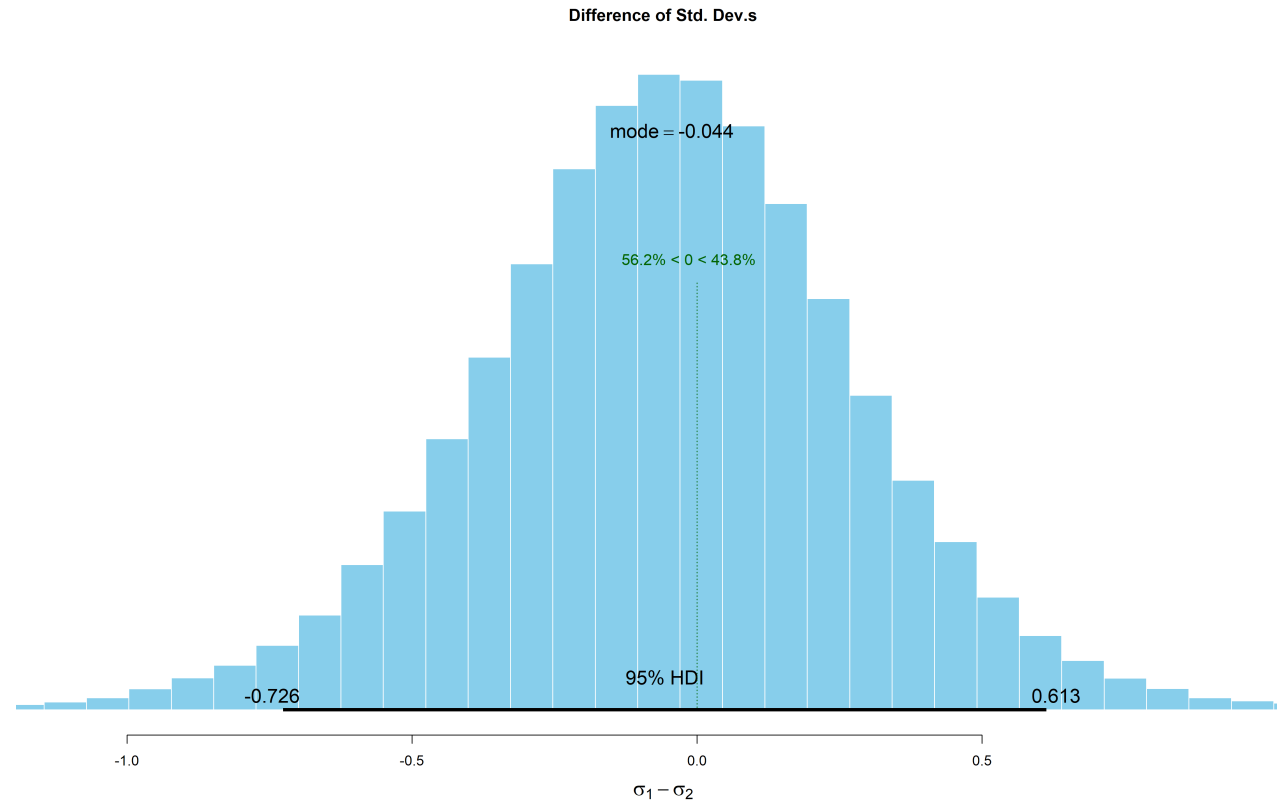

Figure 66. Posterior distribution of the difference in standard deviation for number of letters for the trained novel name targets preceded by semantically related and unrelated primes. 


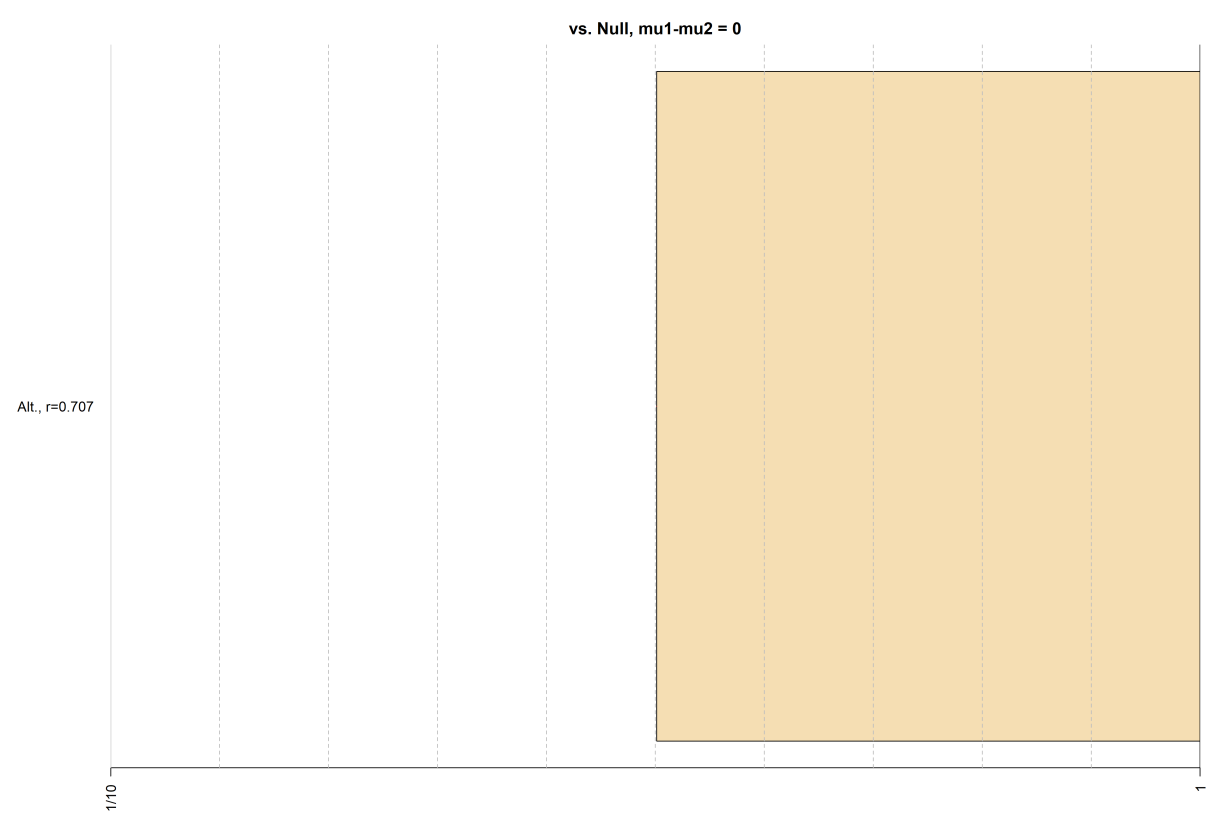

Figure 67. Graphical representation of the Bayes factor (denominator model: $\mu_{1}-\mu_{2}=$ $0)$.
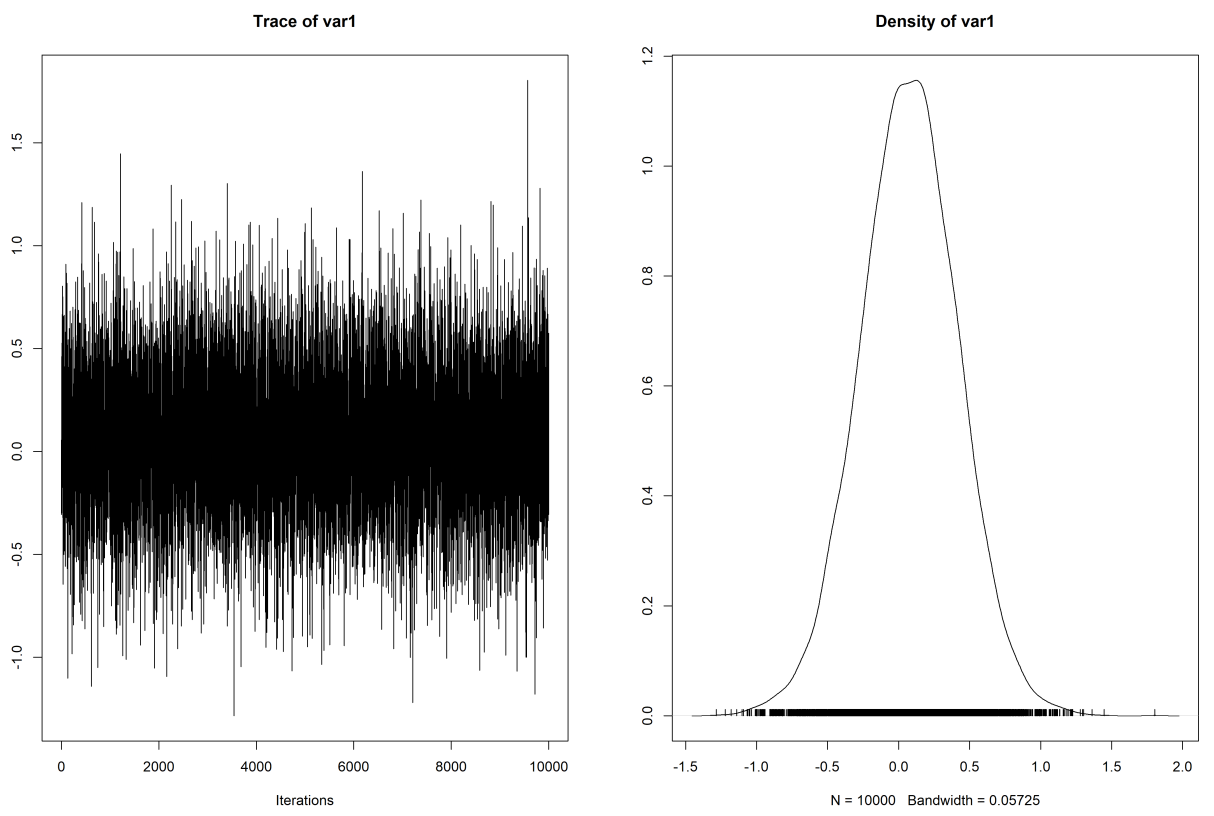

Figure 68. Sampling diagnostics of the posterior for the numerator model. 
Unconstrained bigram frequency for the trained novel name targets preceded by semantically related and unrelated primes

\begin{tabular}{lccccccr}
\hline & Mean & Median & Mode & $\begin{array}{c}95 \% \text { HDI } \\
\text { lower }\end{array}$ & $\begin{array}{c}95 \% \text { HDI } \\
\text { upper }\end{array}$ & $\begin{array}{c}\text { Comp. } \\
\text { value }\end{array}$ & $\begin{array}{r}\text { Comp. } \\
\text { value }\end{array}$ \\
\hline$\mu_{1}$ & 18396.333 & 18322.508 & 18422.673 & 12940.487 & 23856.790 & & \\
$\mu_{2}$ & 17115.268 & 17078.808 & 17252.199 & 11327.788 & 23008.084 & & \\
$\mu_{\text {diff }}$ & 1281.065 & 1306.352 & 1653.241 & -6615.244 & 9091.350 & 0 & 63.100 \\
$\sigma_{1}$ & 10741.917 & 10240.574 & 9125.921 & 5460.724 & 17035.873 & & \\
$\sigma_{2}$ & 11173.448 & 10947.870 & 10688.253 & 6549.390 & 16027.058 & & \\
$\sigma_{d i f f}$ & -431.531 & -553.460 & -733.684 & -7502.742 & 6863.500 & 0 & 43.300 \\
$\nu$ & 11.112 & 6.287 & 3.713 & 1.001 & 37.566 & & \\
$\log _{10} \nu$ & 0.859 & 0.798 & 0.688 & 0.231 & 1.637 & & \\
effect & 0.128 & 0.120 & 0.116 & -0.598 & 0.861 & 0 & 63.100 \\
size & & & & & & & \\
\hline
\end{tabular}

Table 25. Bayesian estimation for unconstrained bigram frequency for the trained novel name targets preceded by semantically related and unrelated primes.

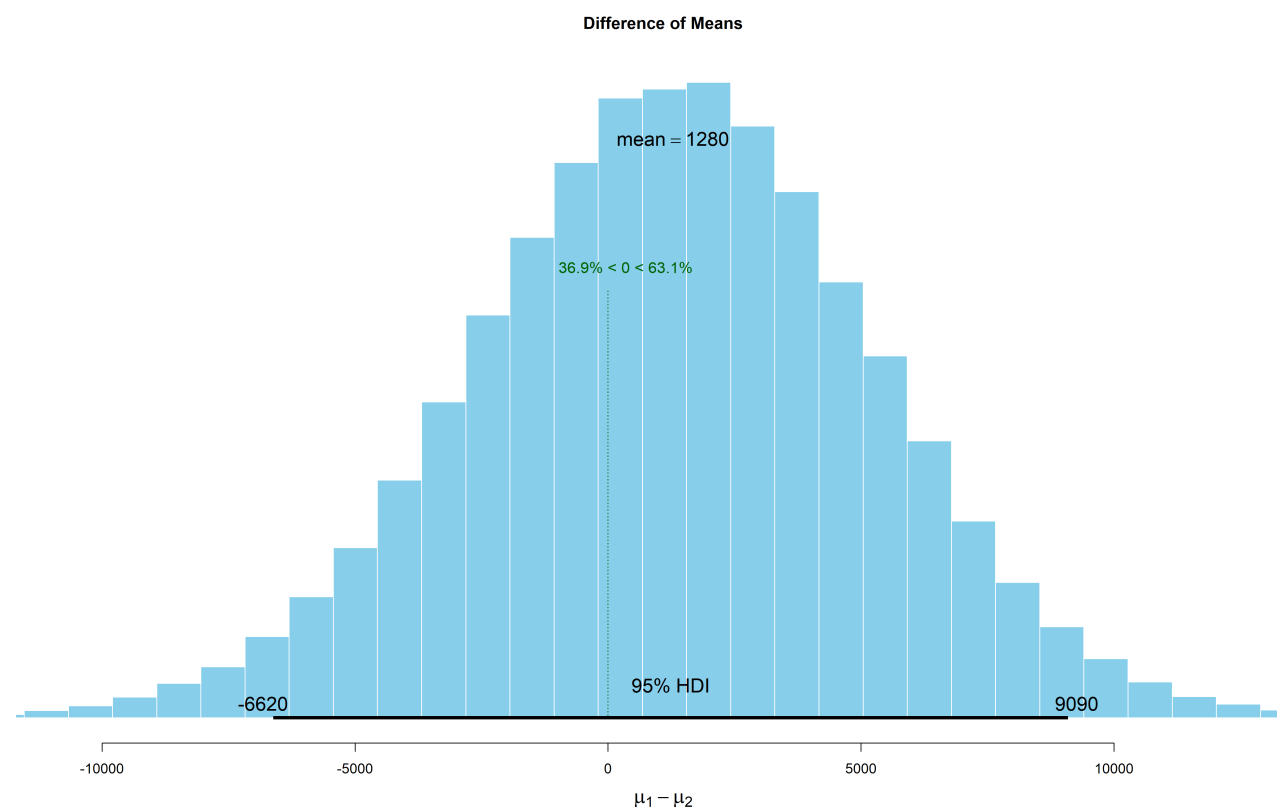

Figure 69. Posterior distribution of the difference in means for unconstrained bigram frequency for the trained novel name targets preceded by semantically related and unrelated primes. 


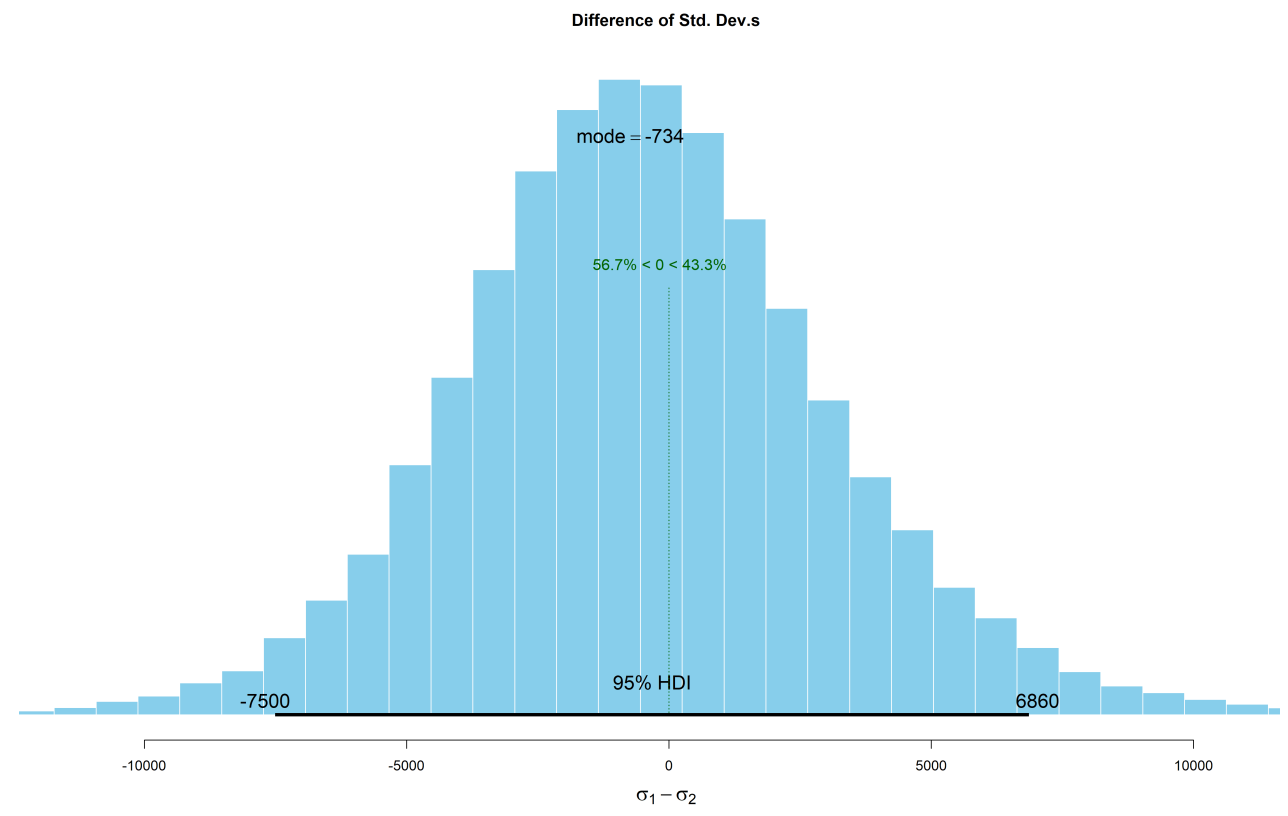

Figure 70. Posterior distribution of the difference in standard deviation for unconstrained bigram frequency for the trained novel name targets preceded by semantically related and unrelated primes.

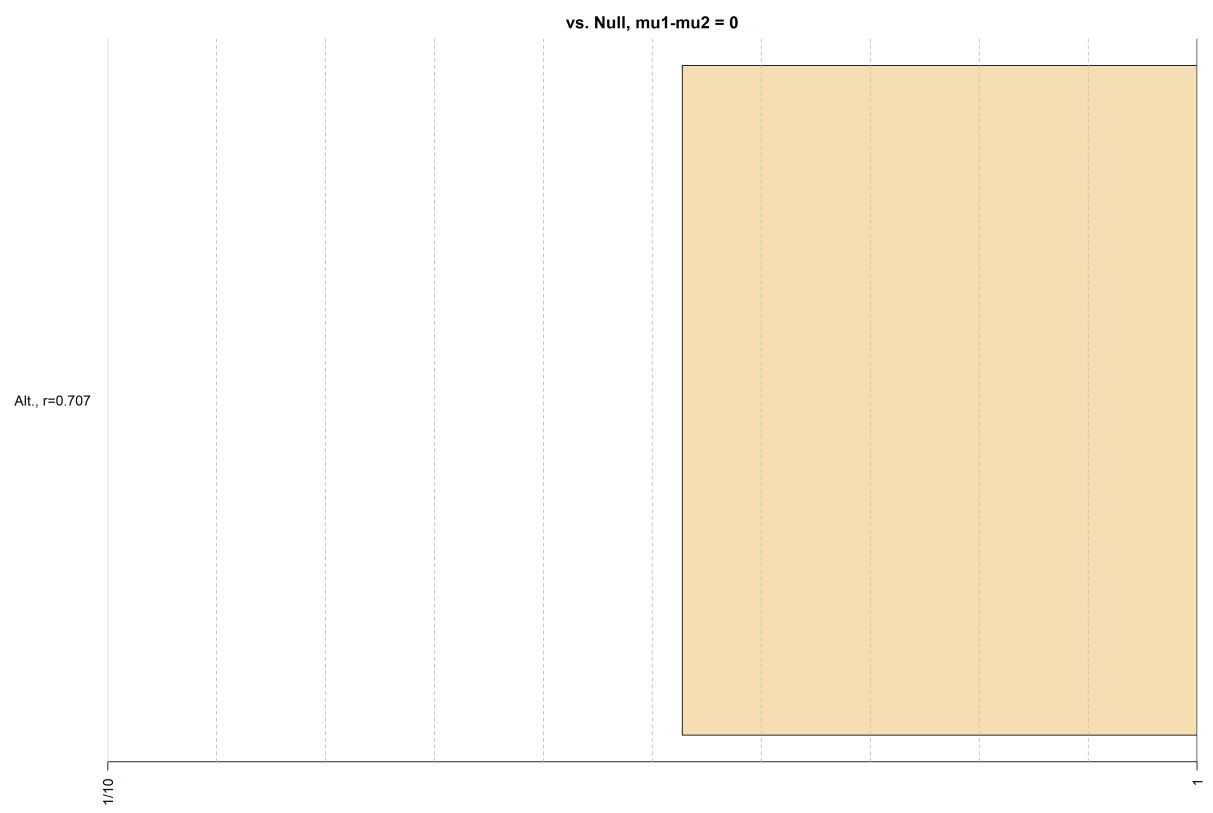

Figure 71. Graphical representation of the Bayes factor (denominator model: $\mu_{1}-\mu_{2}=$ $0)$. 

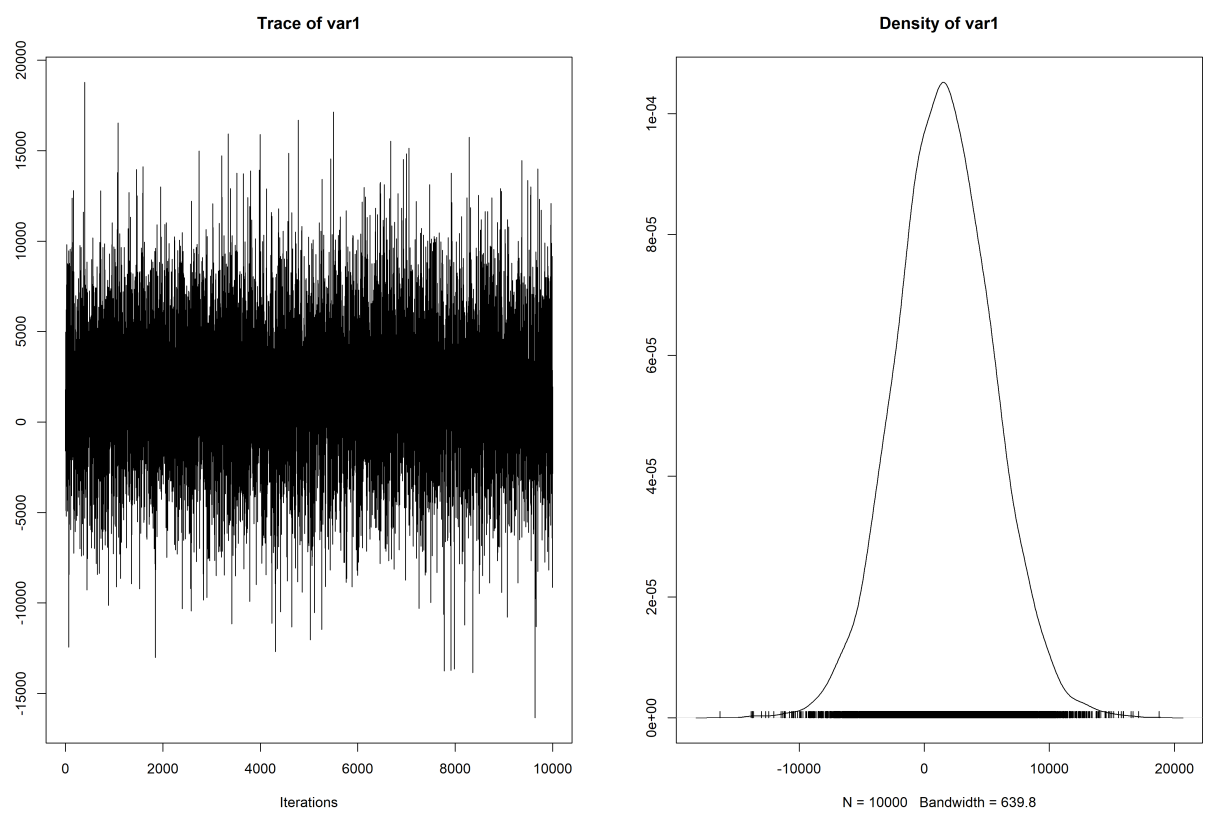

Figure 72. Sampling diagnostics of the posterior for the numerator model.

Constrained bigram frequency for the trained novel name targets preceded by semantically related and unrelated primes

\begin{tabular}{lccccccc}
\hline & Mean & Median & Mode & $\begin{array}{c}95 \% \text { HDI } \\
\text { lower }\end{array}$ & $\begin{array}{c}95 \% \text { HDI } \\
\text { upper }\end{array}$ & $\begin{array}{c}\text { Comp. } \\
\text { value }\end{array}$ & $\begin{array}{r}\%> \\
\text { Comp. } \\
\text { value }\end{array}$ \\
\hline$\mu_{1}$ & 645.998 & 627.223 & 602.835 & 380.995 & 951.850 & & \\
$\mu_{2}$ & 465.230 & 461.025 & 458.827 & 275.193 & 667.340 & & \\
$\mu_{\text {diff }}$ & 180.767 & 170.586 & 147.278 & -150.661 & 532.170 & 0 & 87.400 \\
$\sigma_{1}$ & 464.785 & 429.514 & 374.439 & 155.250 & 842.770 & & \\
$\sigma_{2}$ & 335.388 & 318.006 & 285.159 & 152.301 & 557.920 & & \\
$\sigma_{\text {diff }}$ & 129.397 & 108.906 & 79.669 & -240.984 & 538.660 & 0 & 74.400 \\
$\nu$ & 1.626 & 1.460 & 1.164 & 1.000 & 2.750 & & \\
$\log _{10} \nu$ & 0.188 & 0.164 & 0.010 & 0.000 & 0.440 & & \\
effect size & 0.450 & 0.442 & 0.436 & -0.346 & 1.230 & 0 & 87.400 \\
\hline
\end{tabular}

Table 26. Bayesian estimation for constrained bigram frequency for the trained novel name targets preceded by semantically related and unrelated primes. 


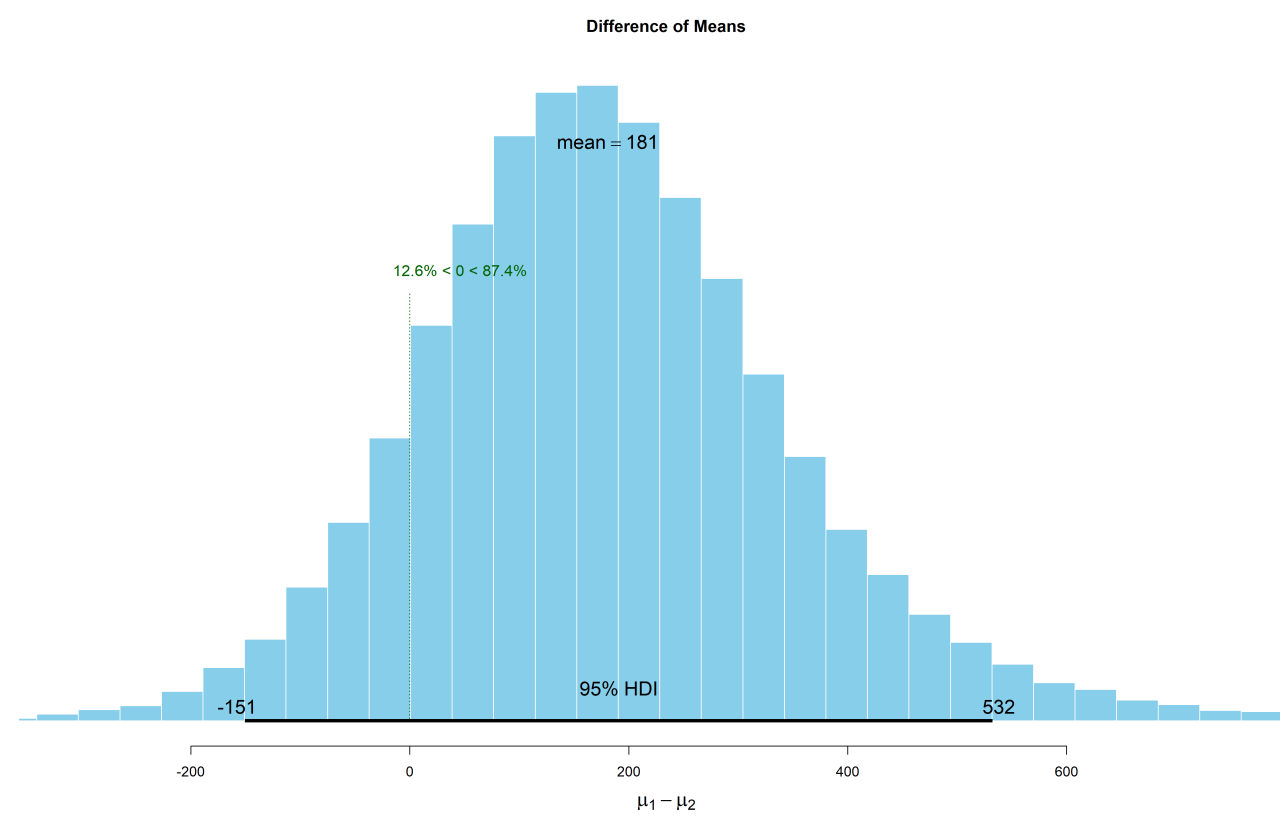

Figure 73. Posterior distribution of the difference in means for constrained bigram frequency for the trained novel name targets preceded by semantically related and unrelated primes.

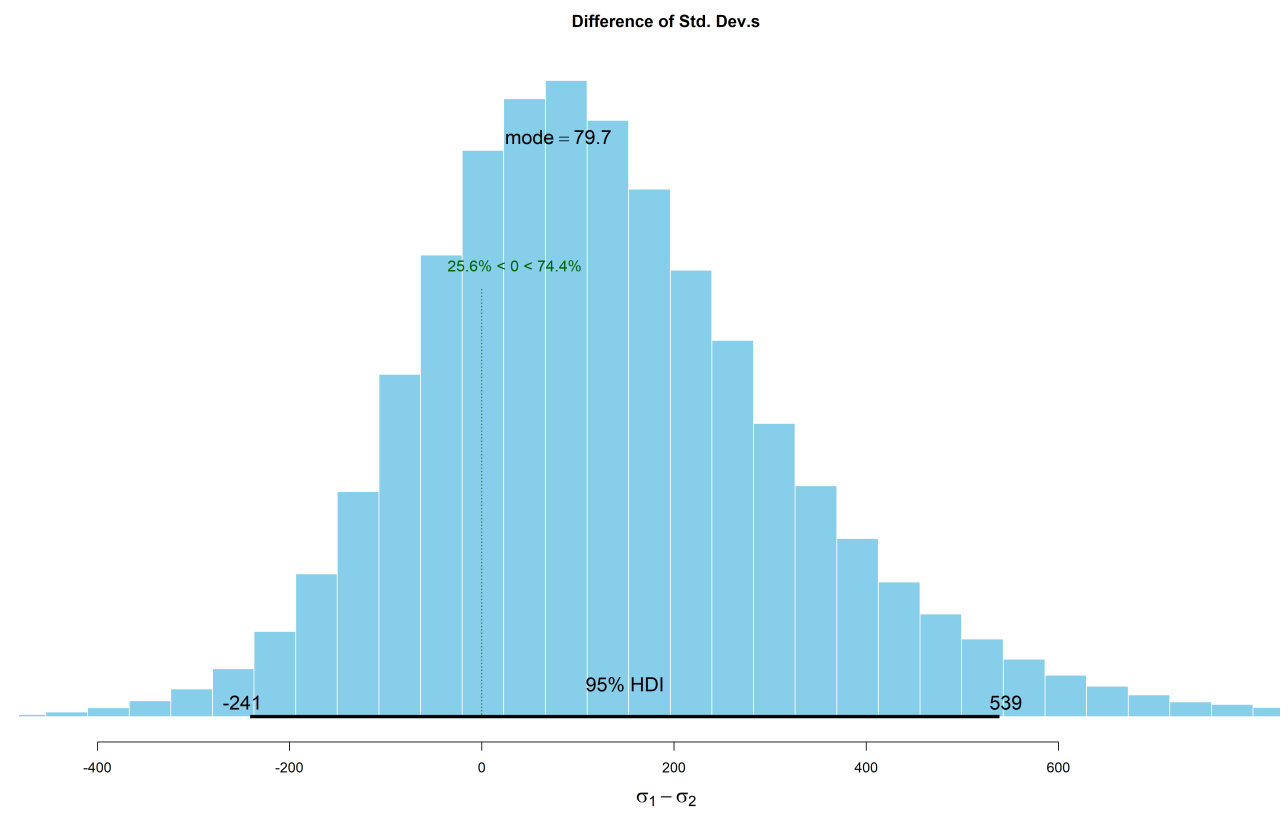

Figure 74. Posterior distribution of the difference in standard deviation for constrained bigram frequency for the trained novel name targets preceded by semantically related and unrelated primes. 


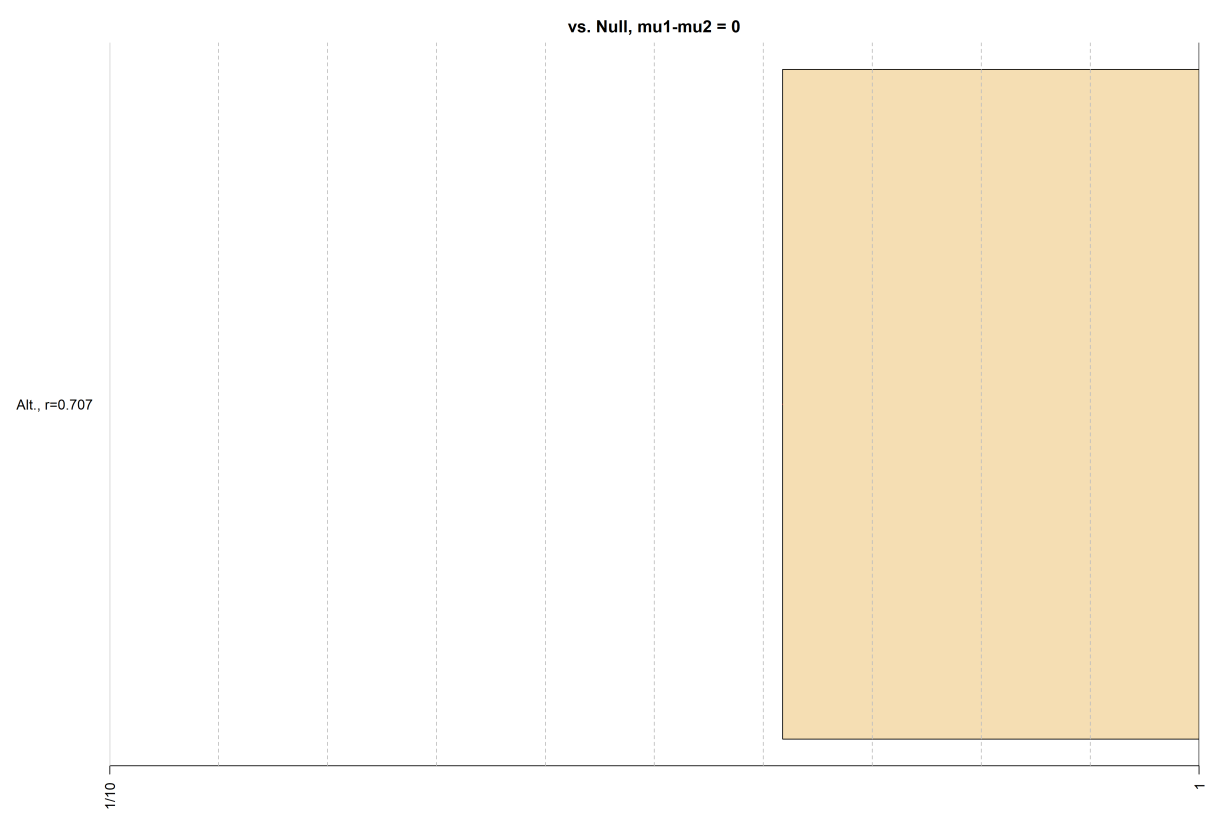

Figure 75. Graphical representation of the Bayes factor (denominator model: $\mu_{1}-\mu_{2}=$ $0)$.
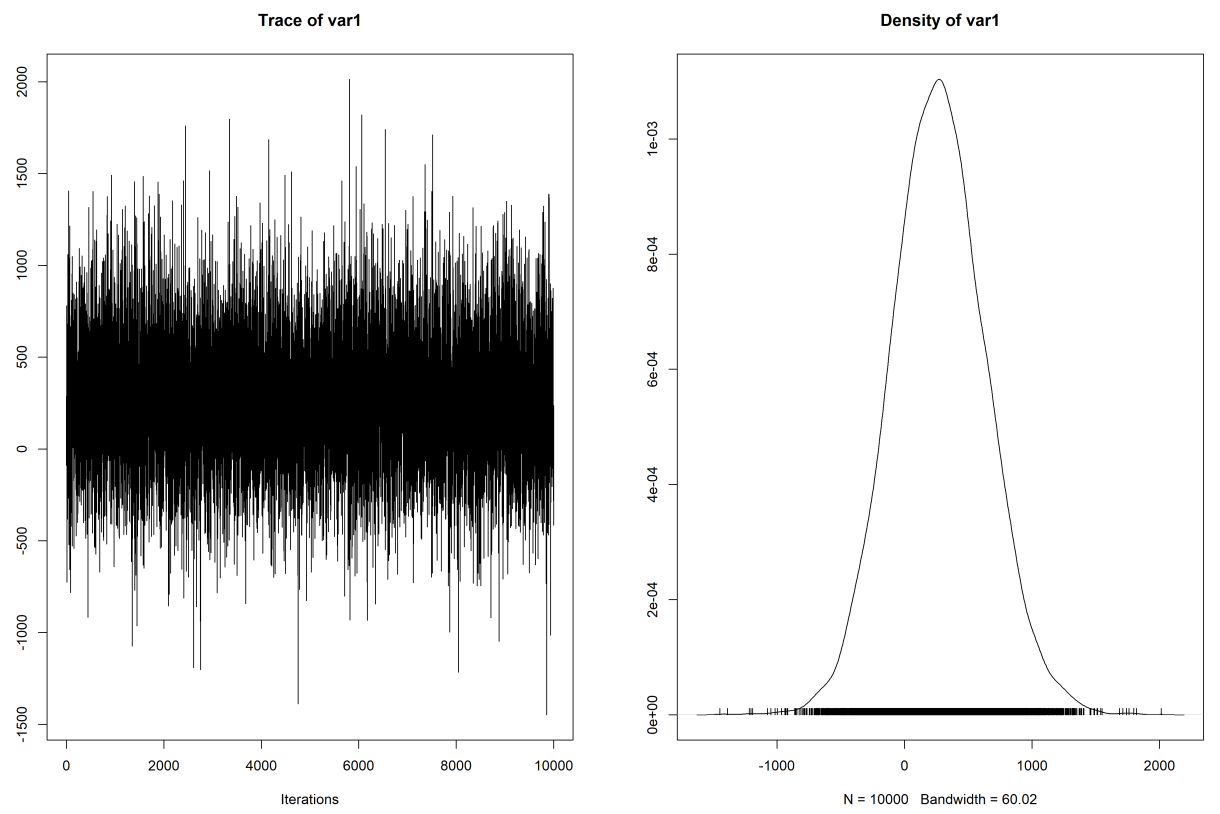

Figure 76. Sampling diagnostics of the posterior for the numerator model. 
Appendix B Stimuli used in the learning phase 


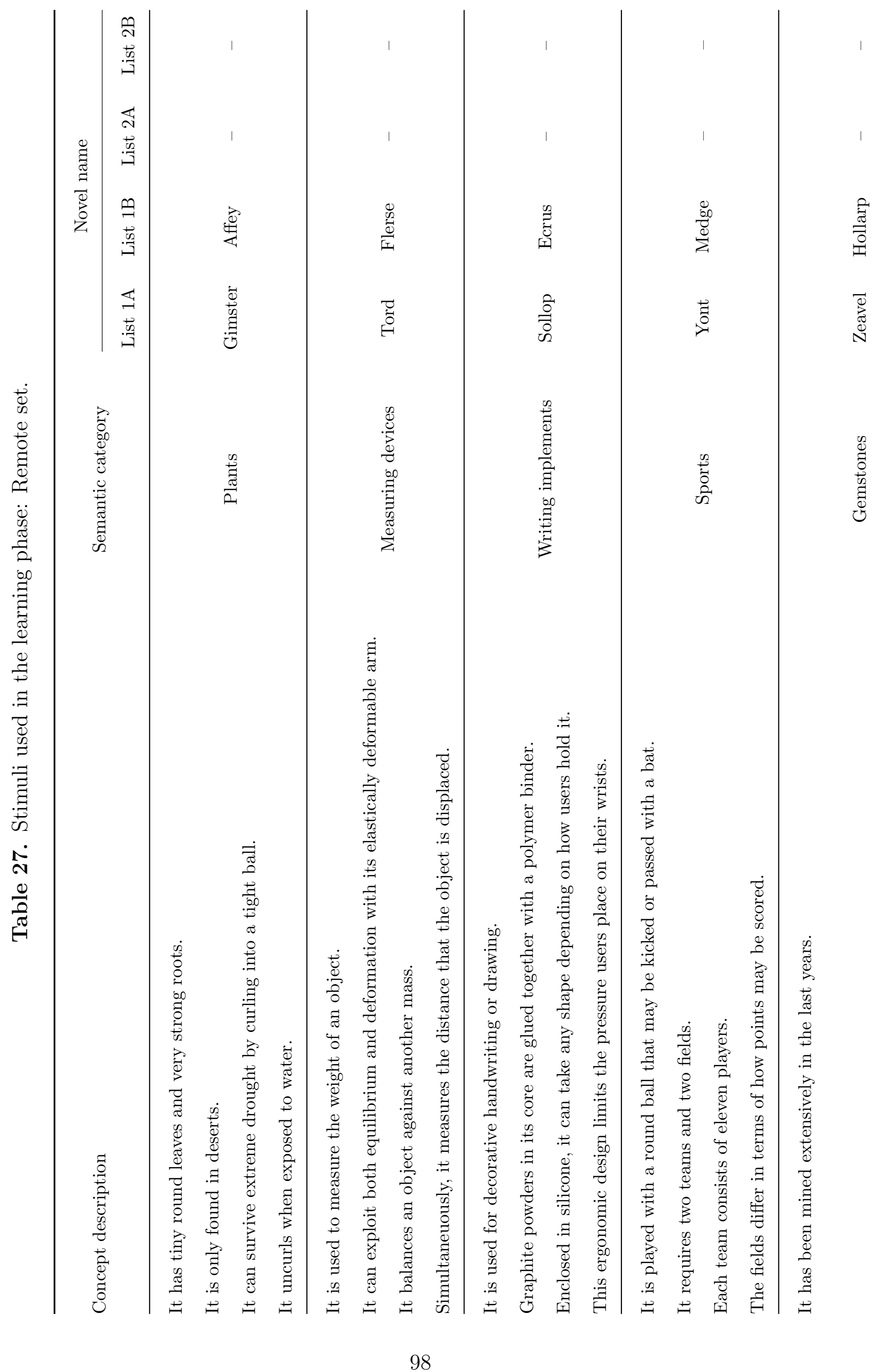




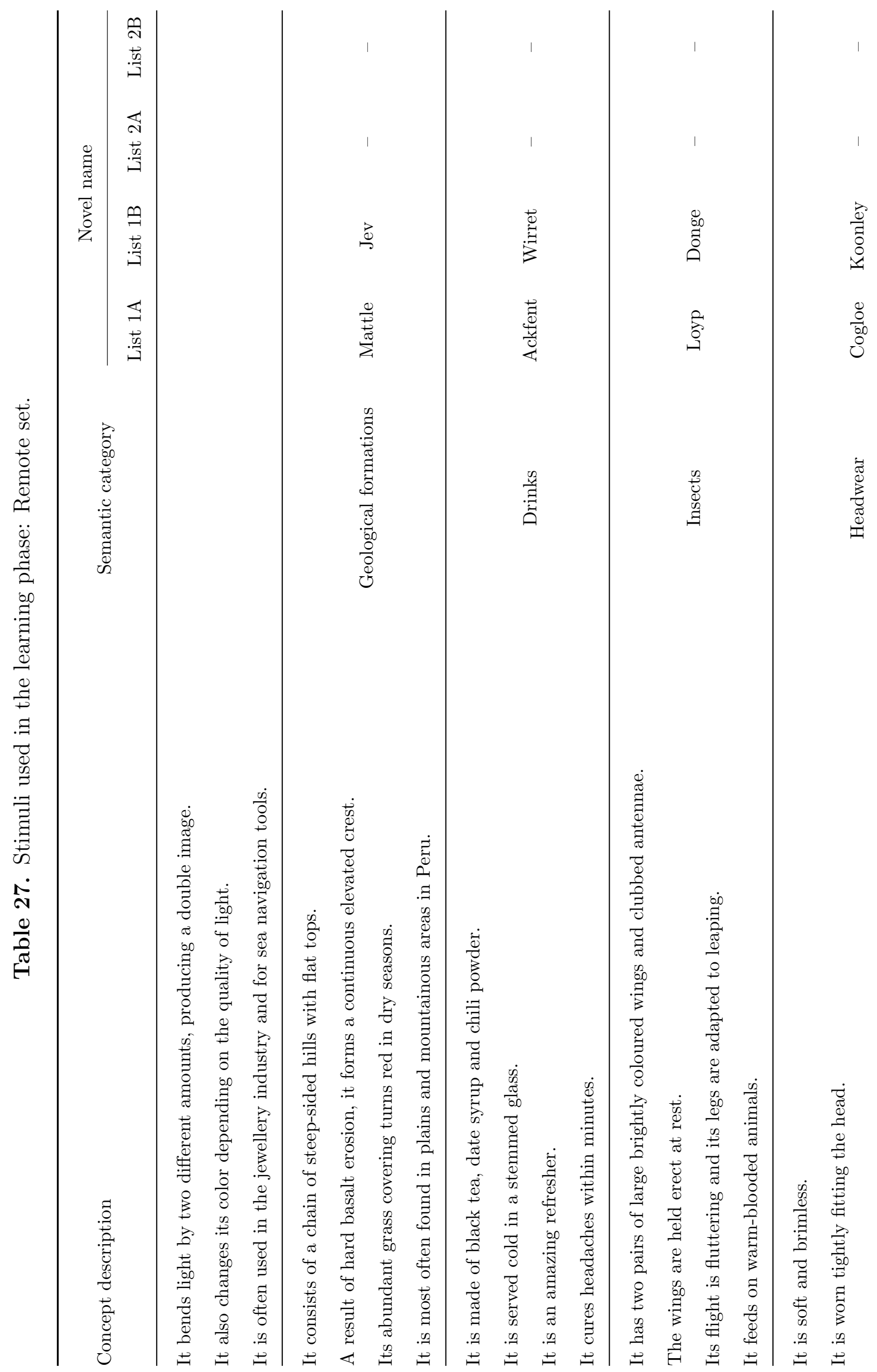




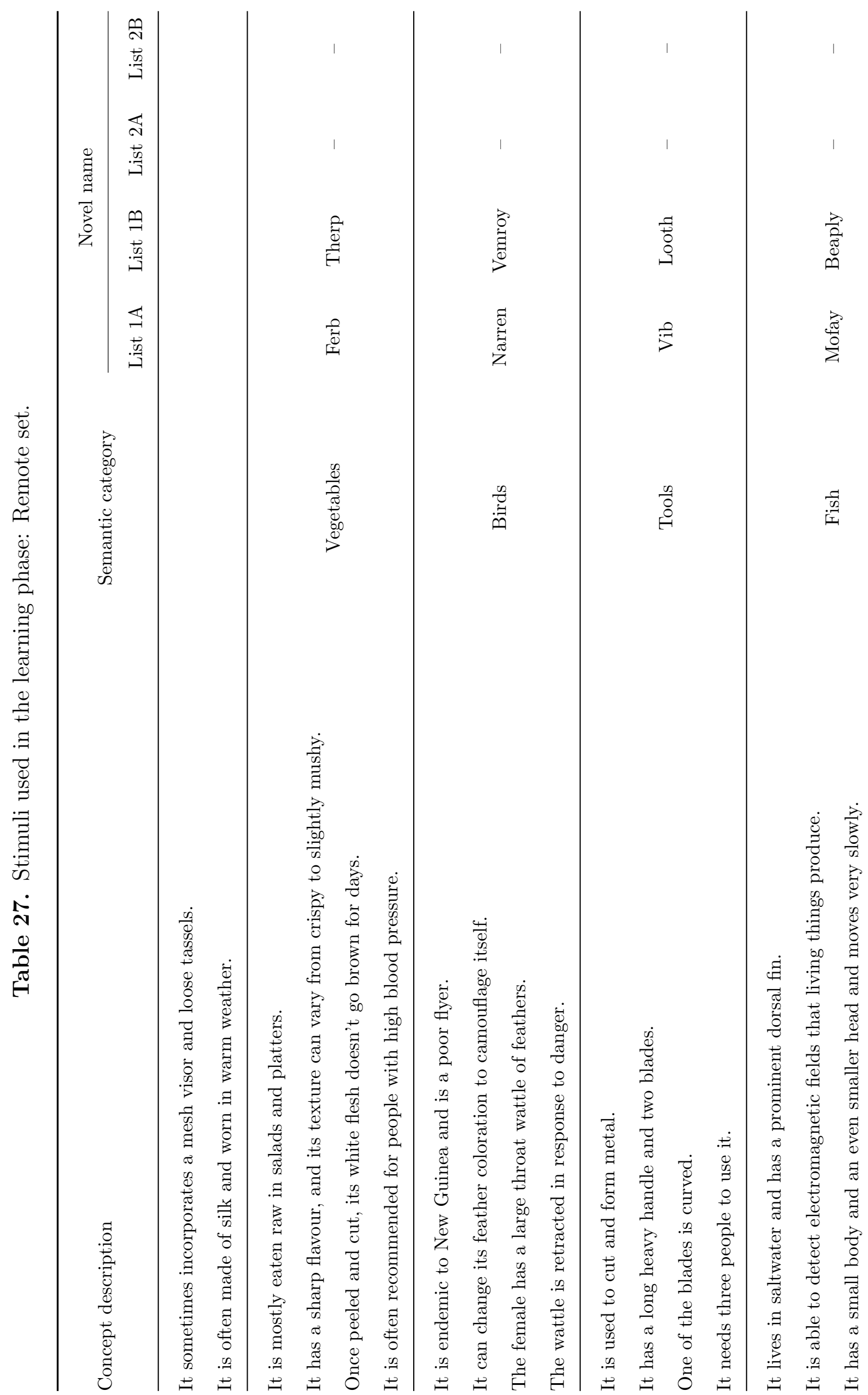




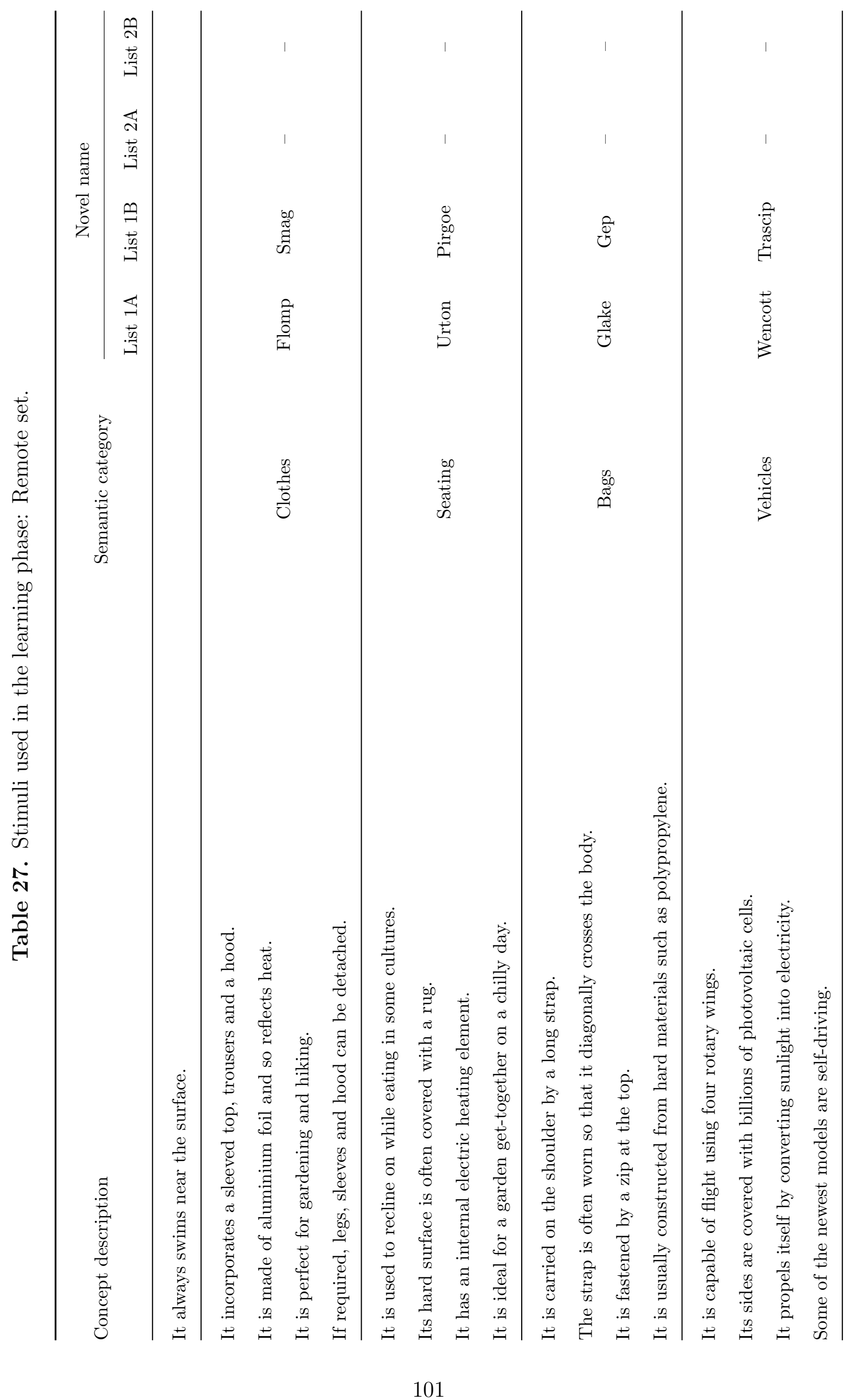




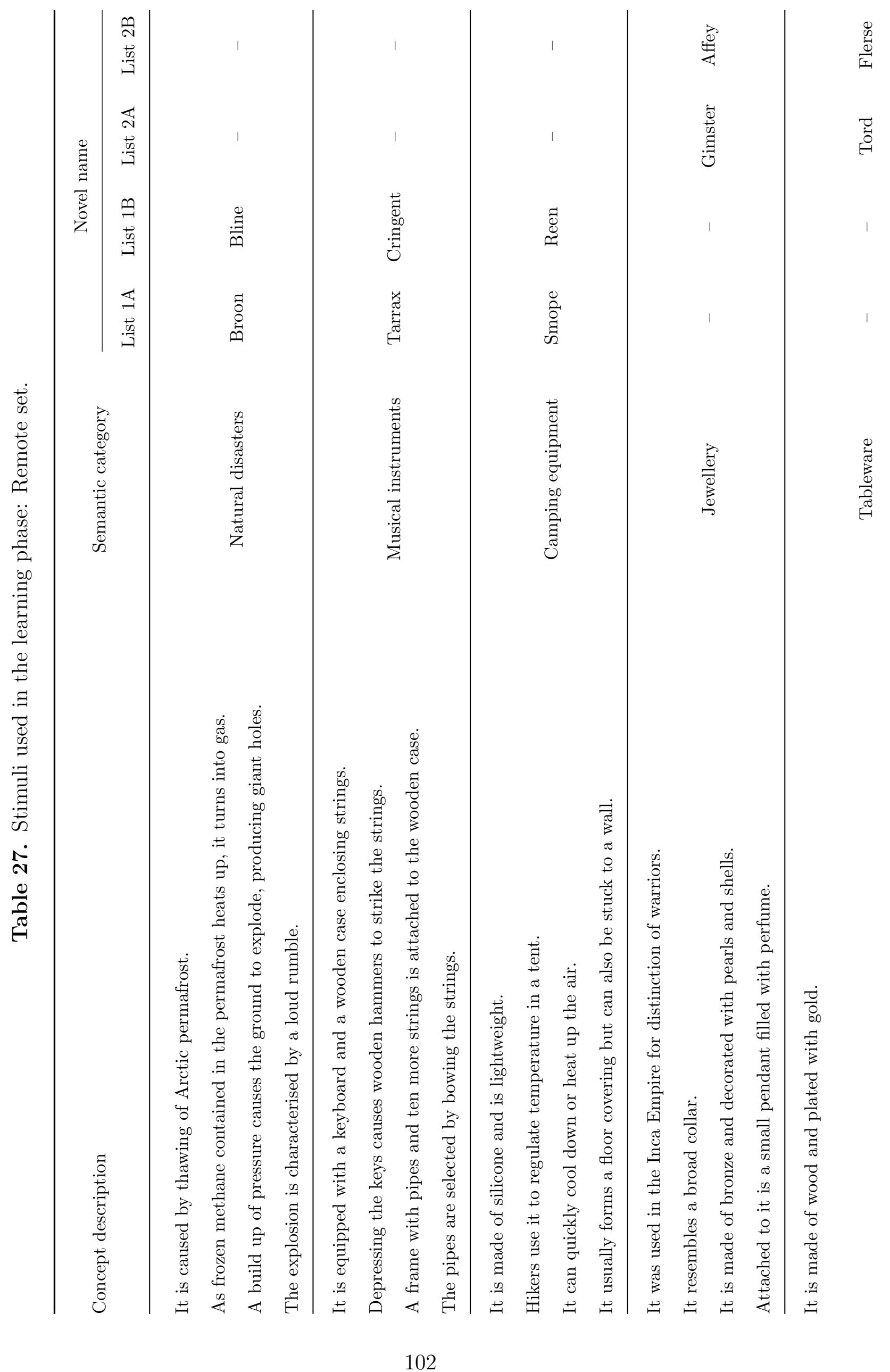




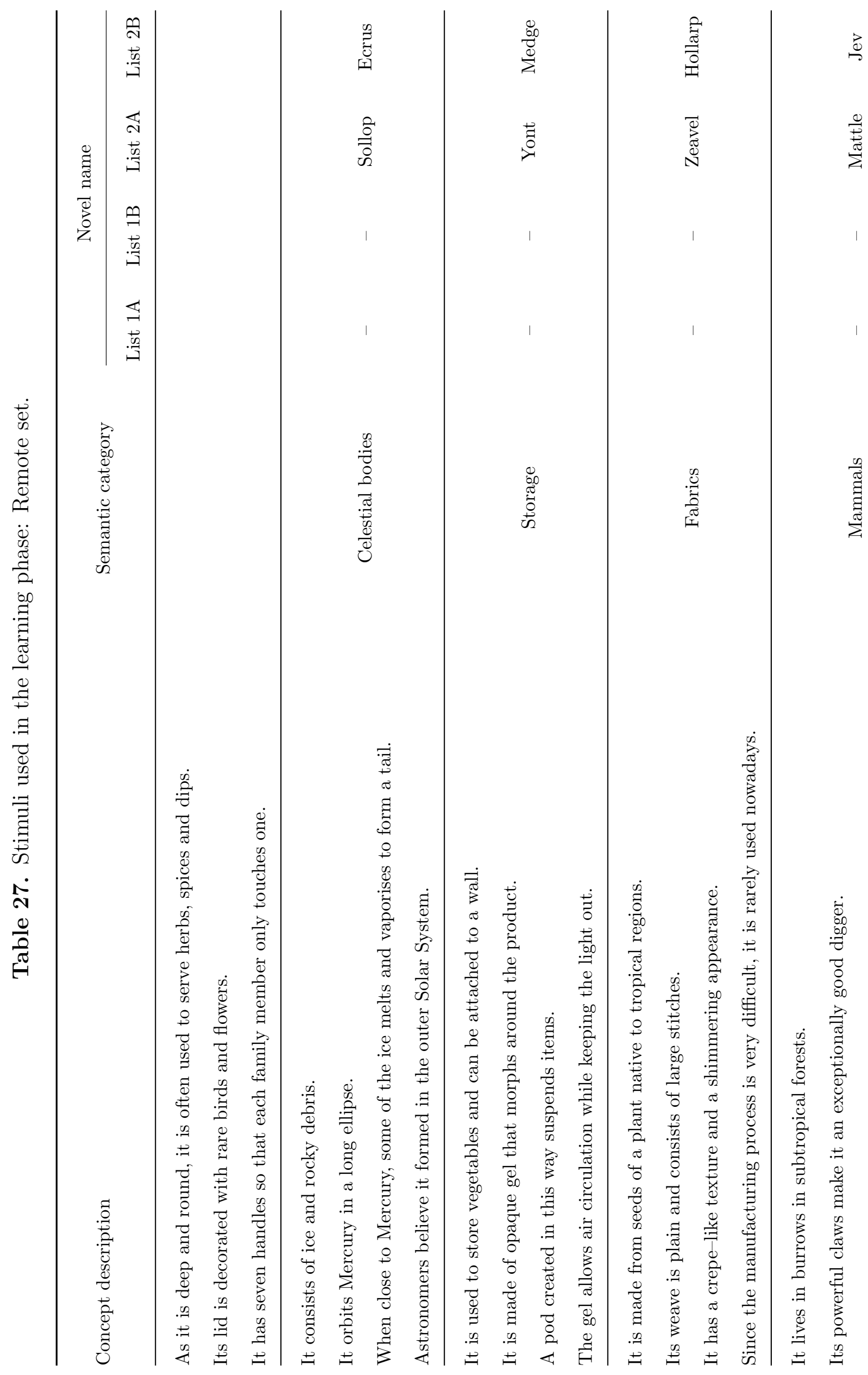




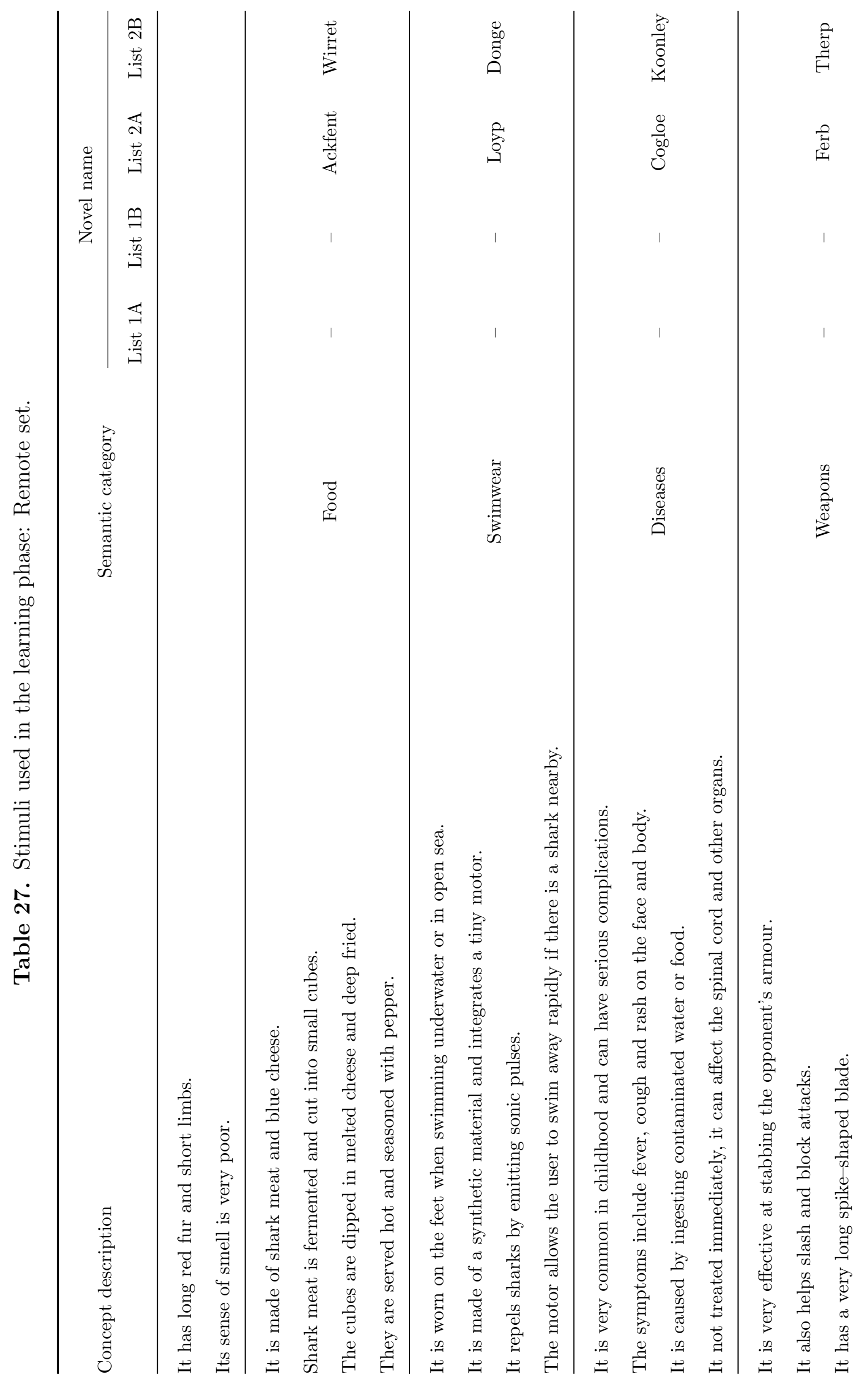




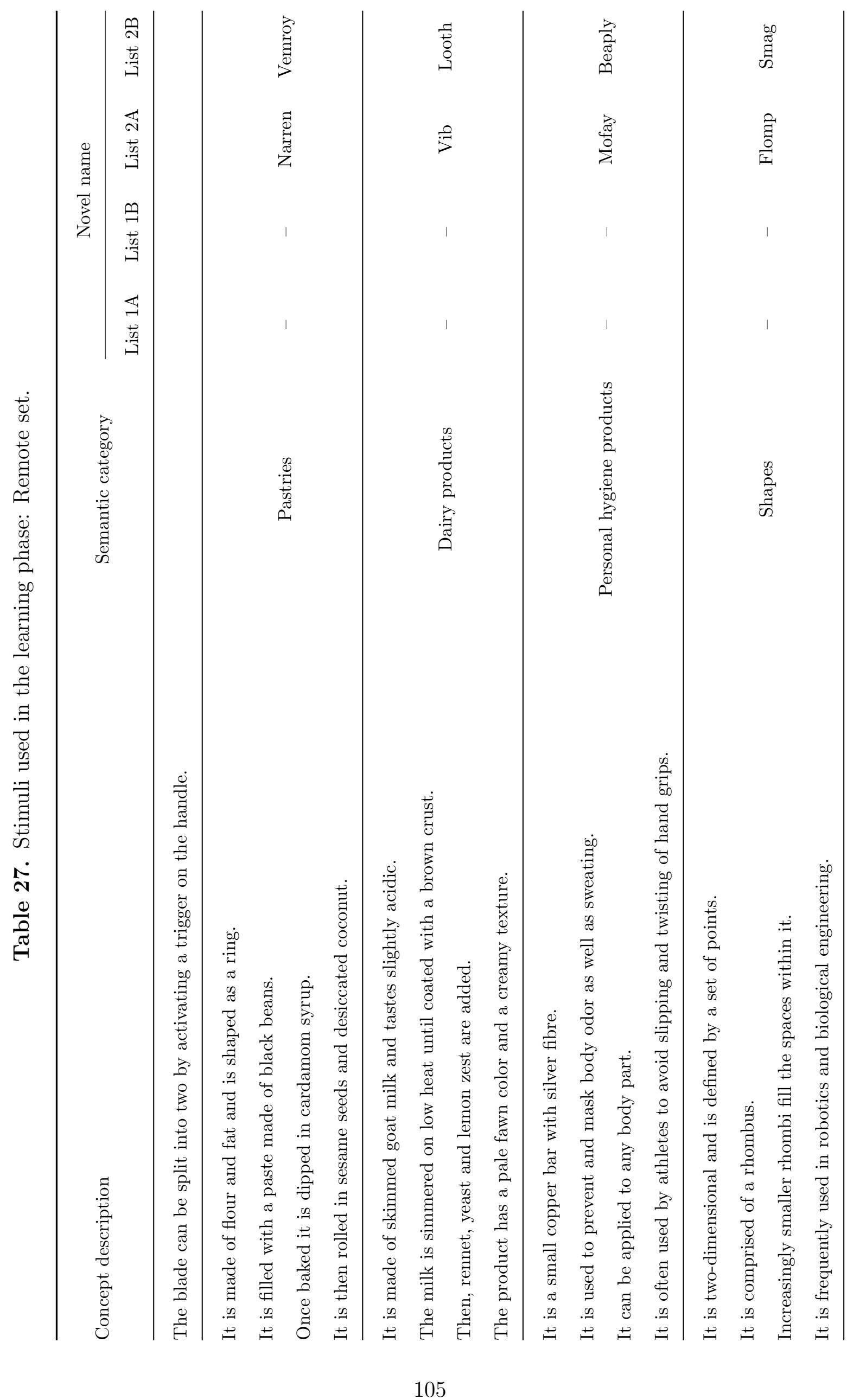




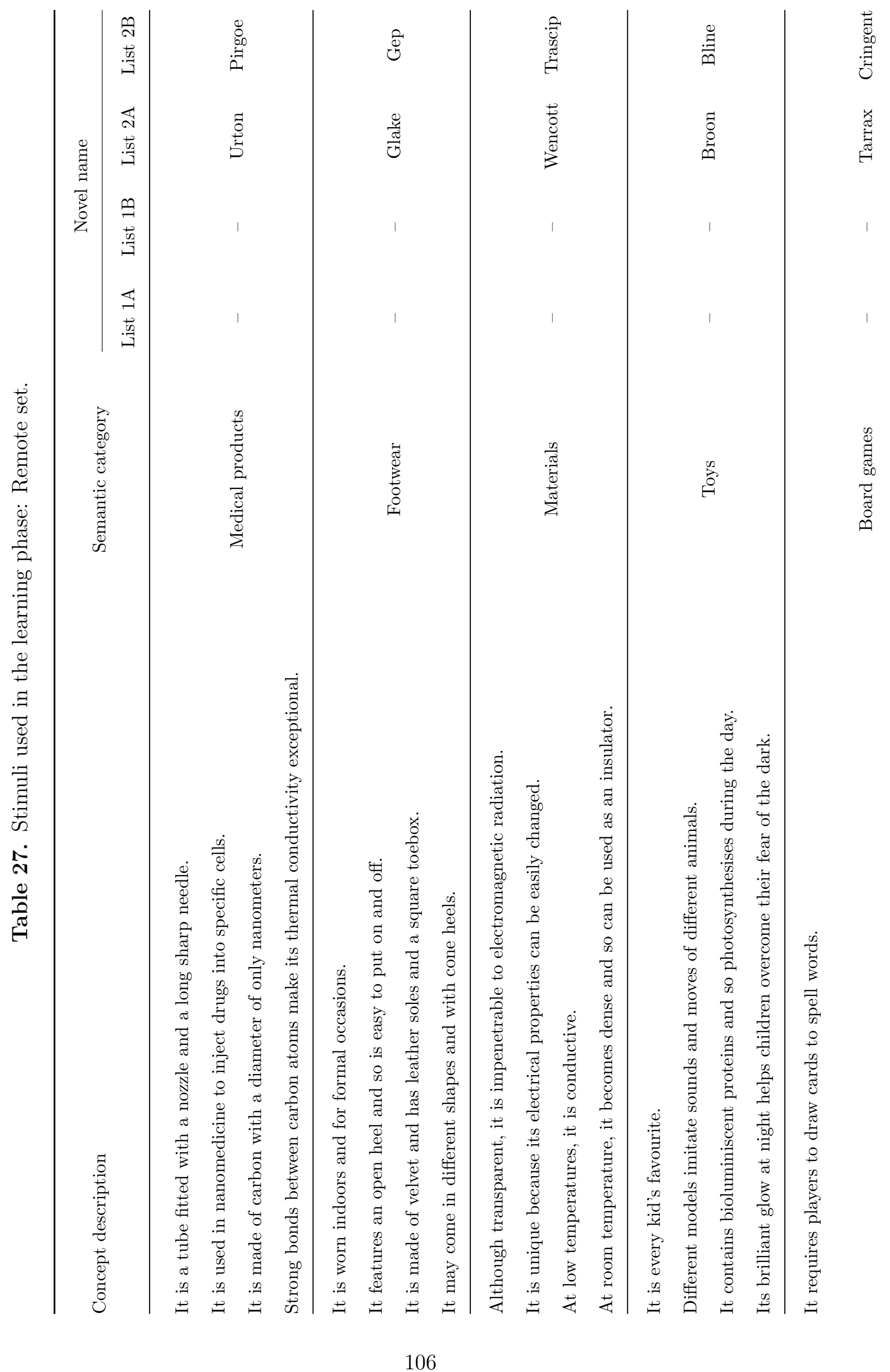




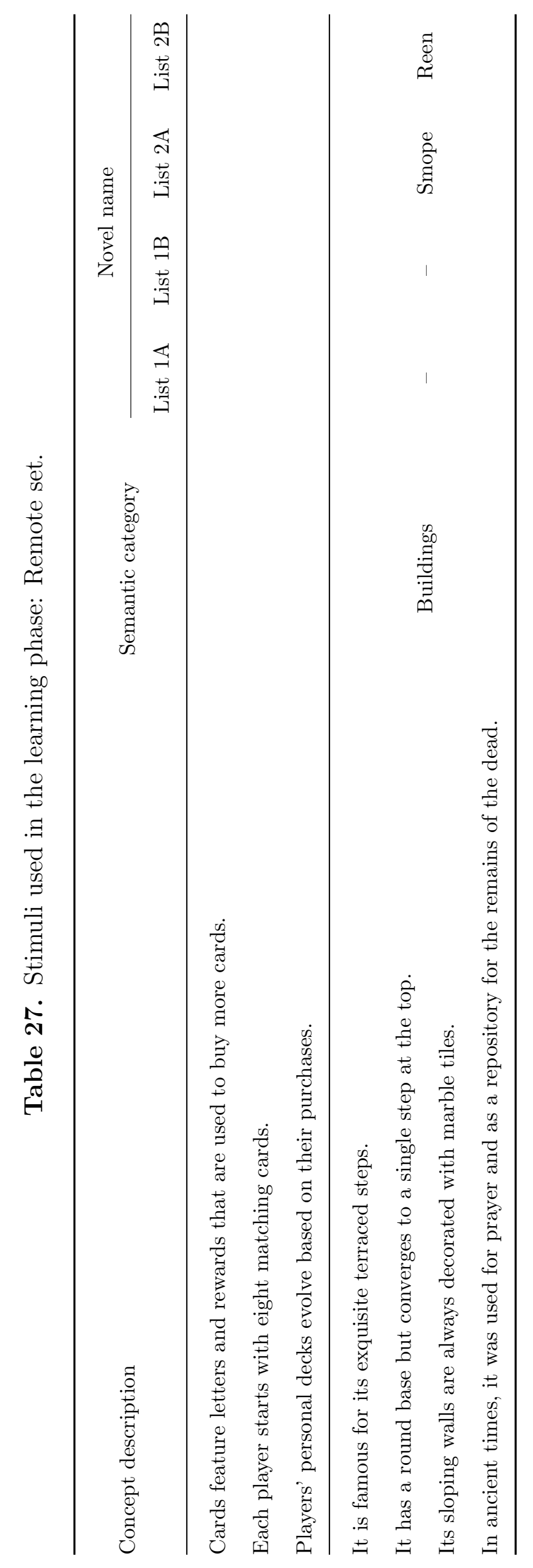




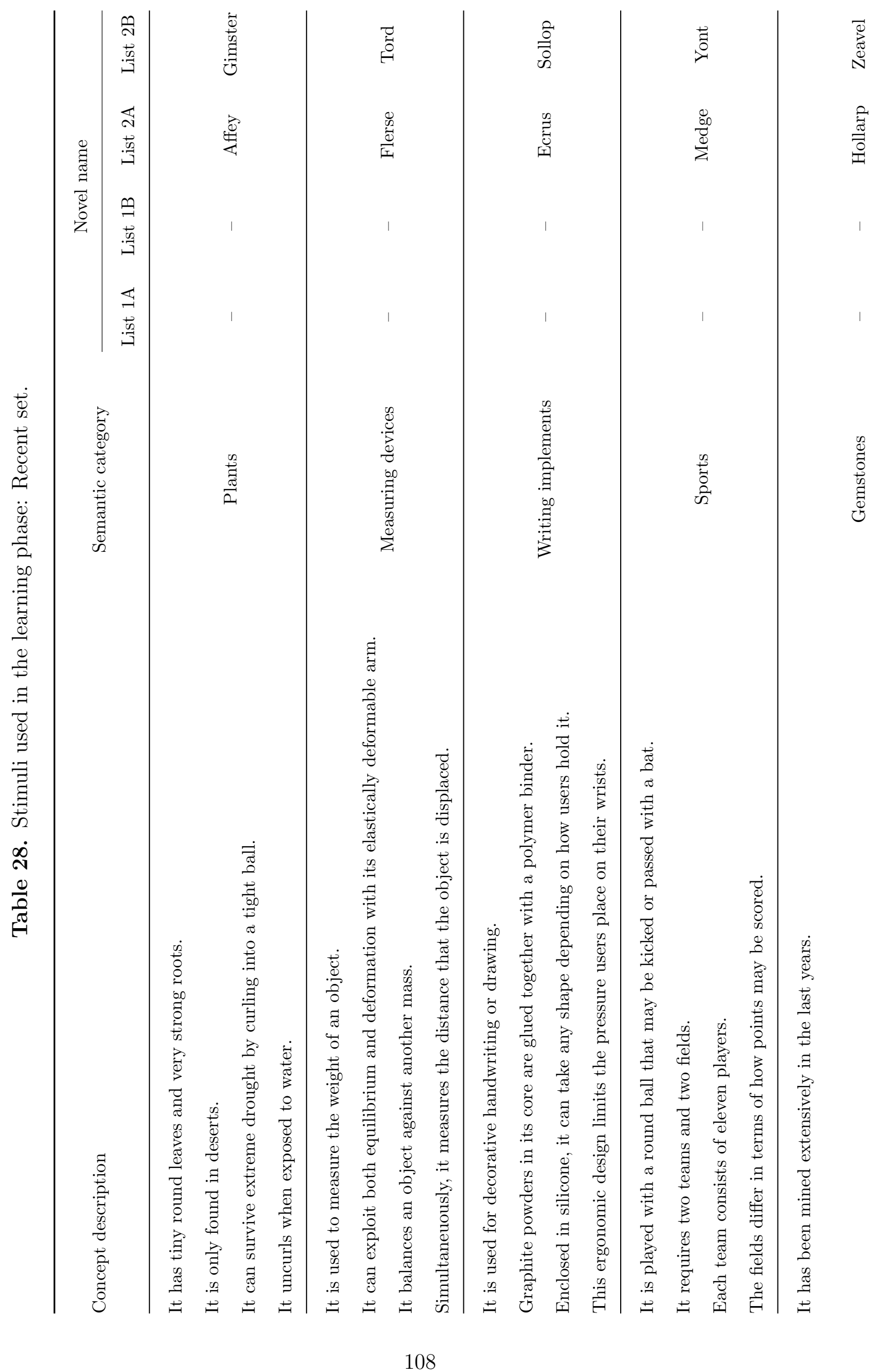




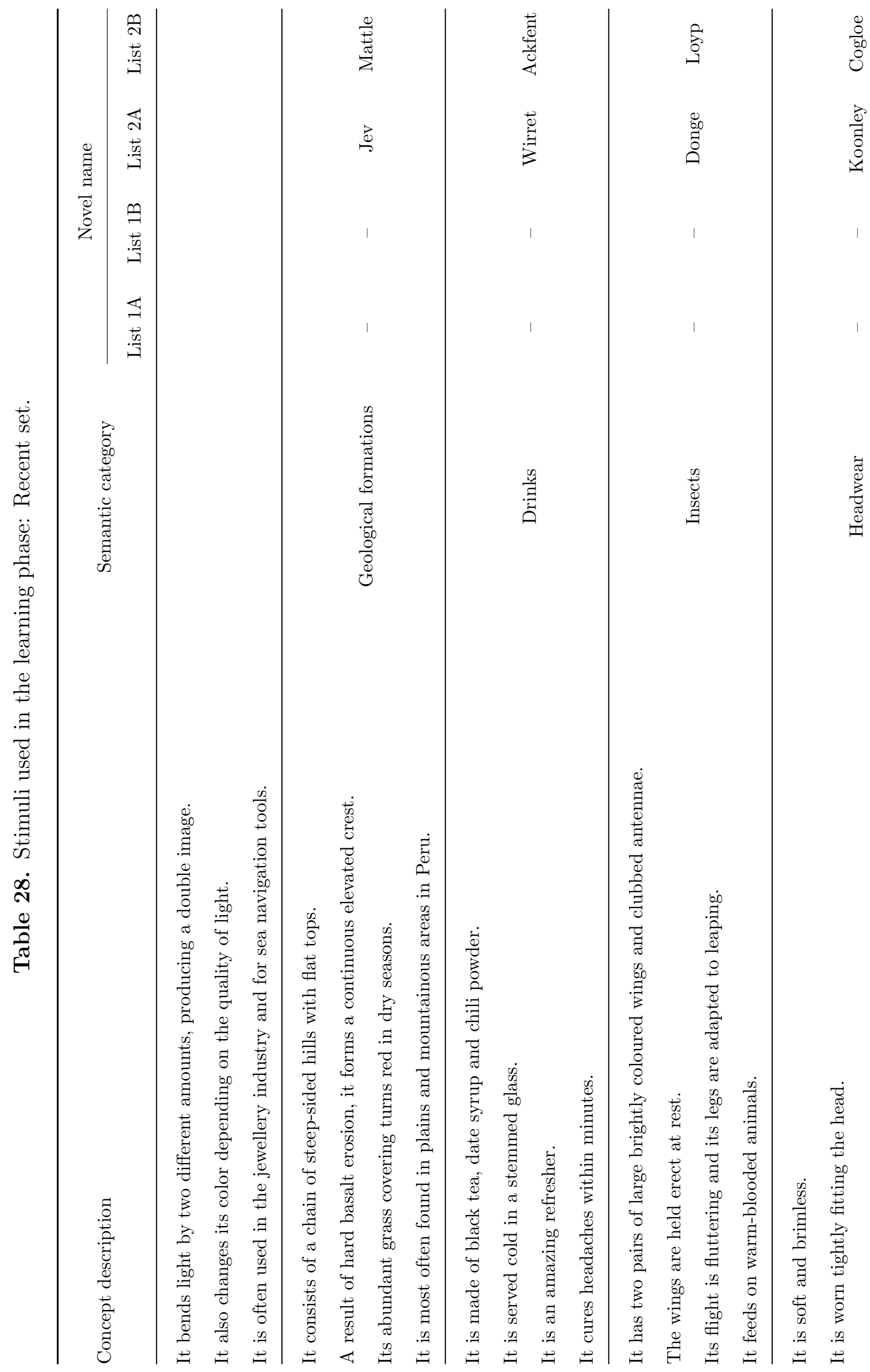




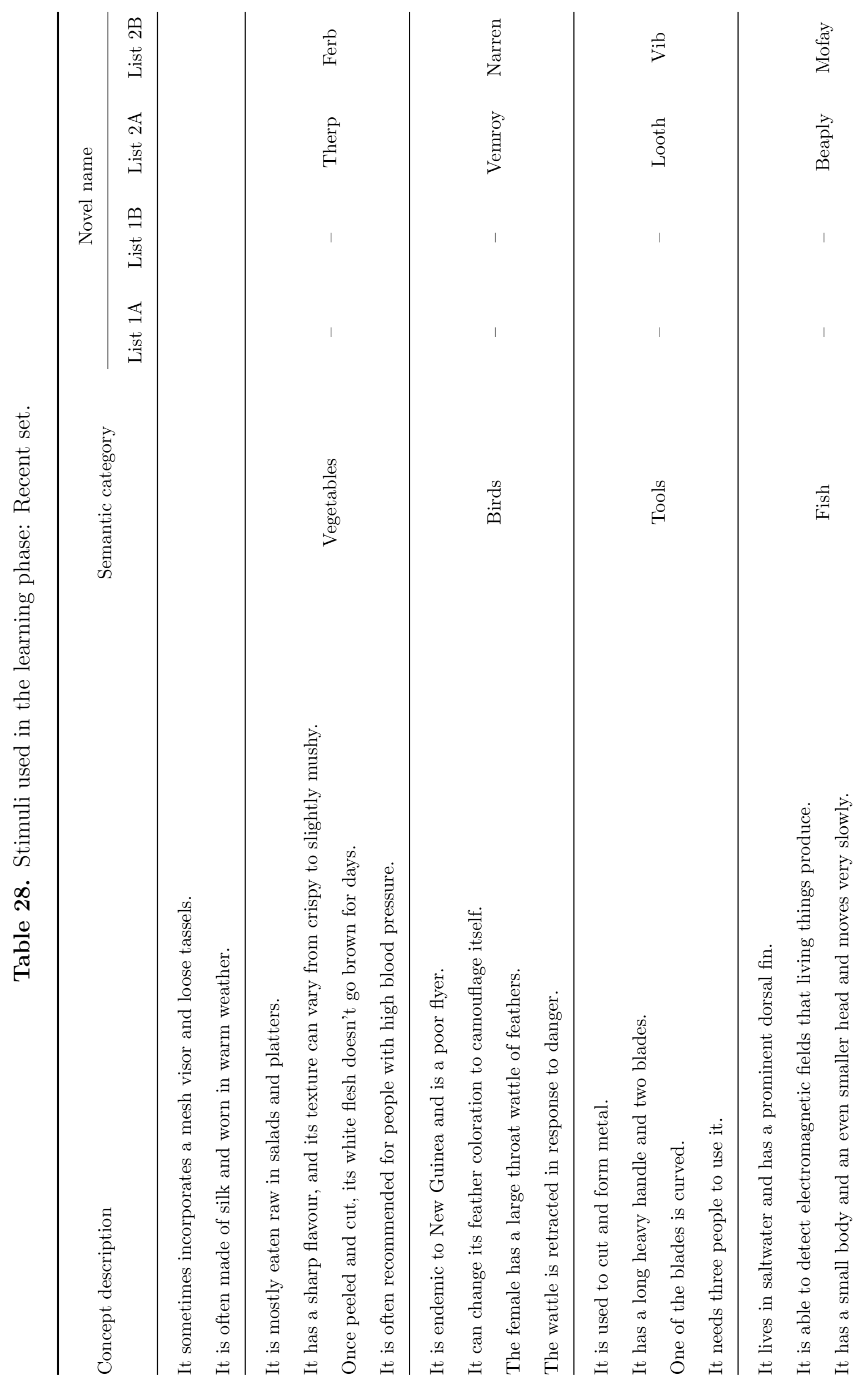




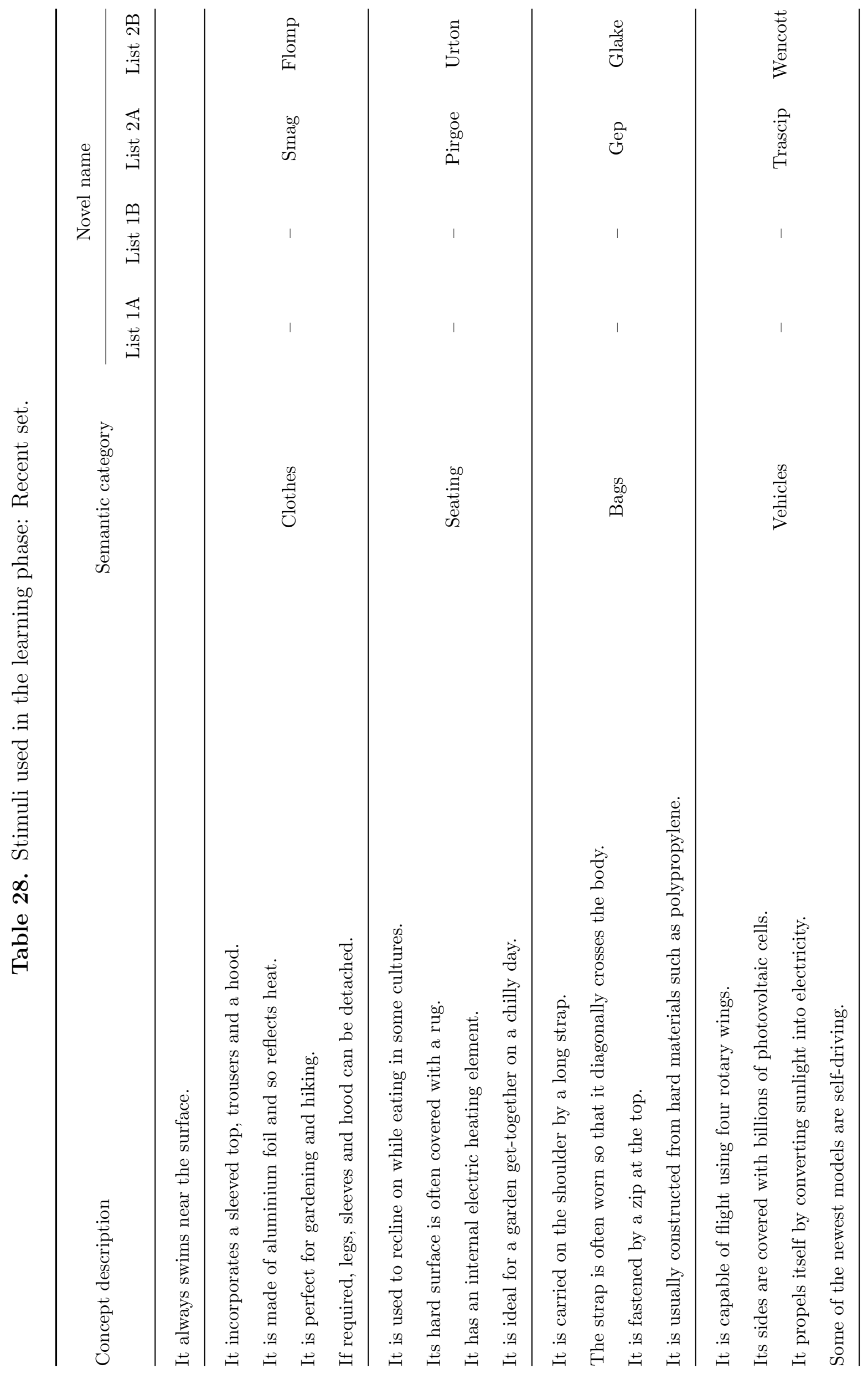




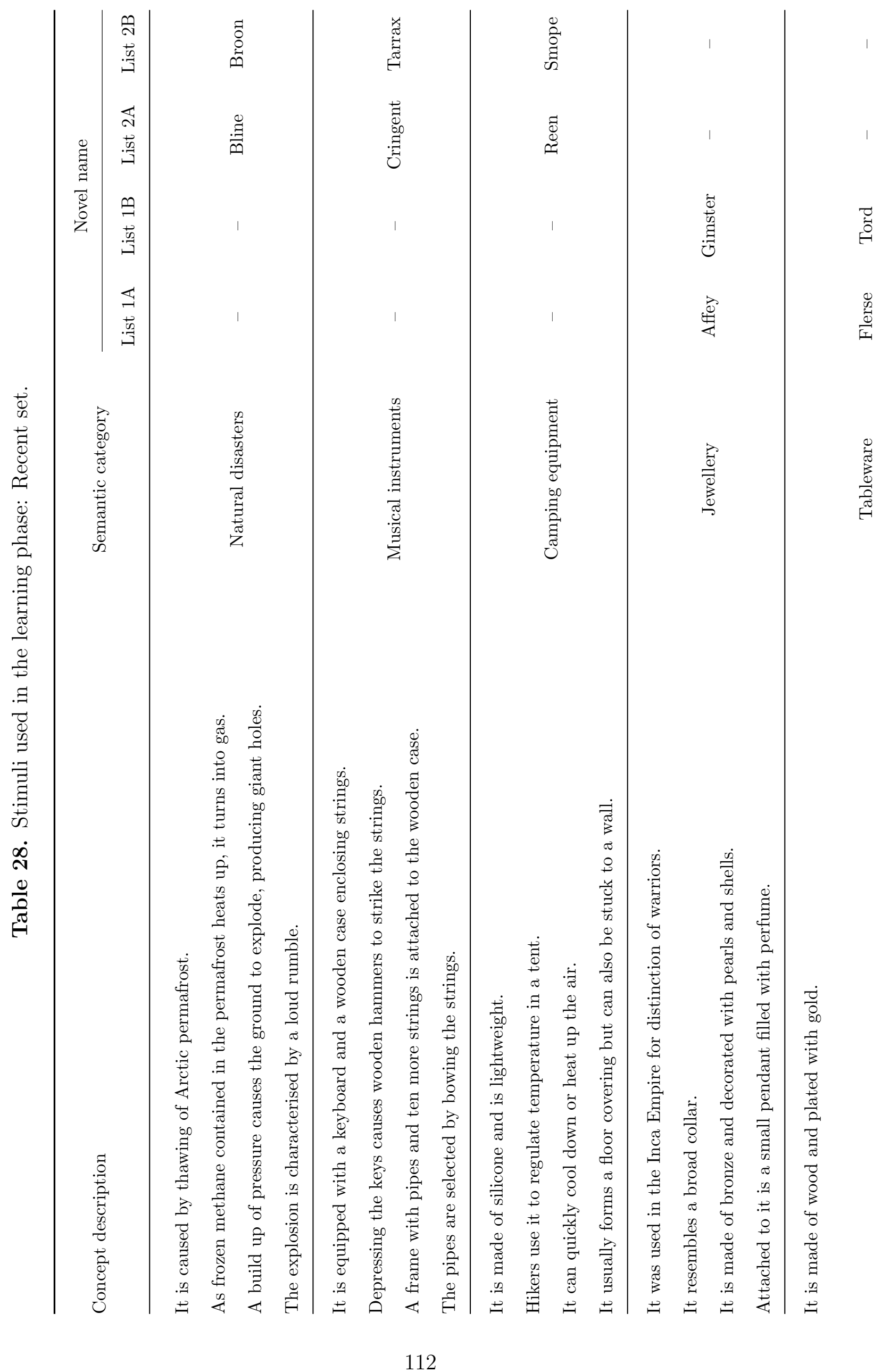




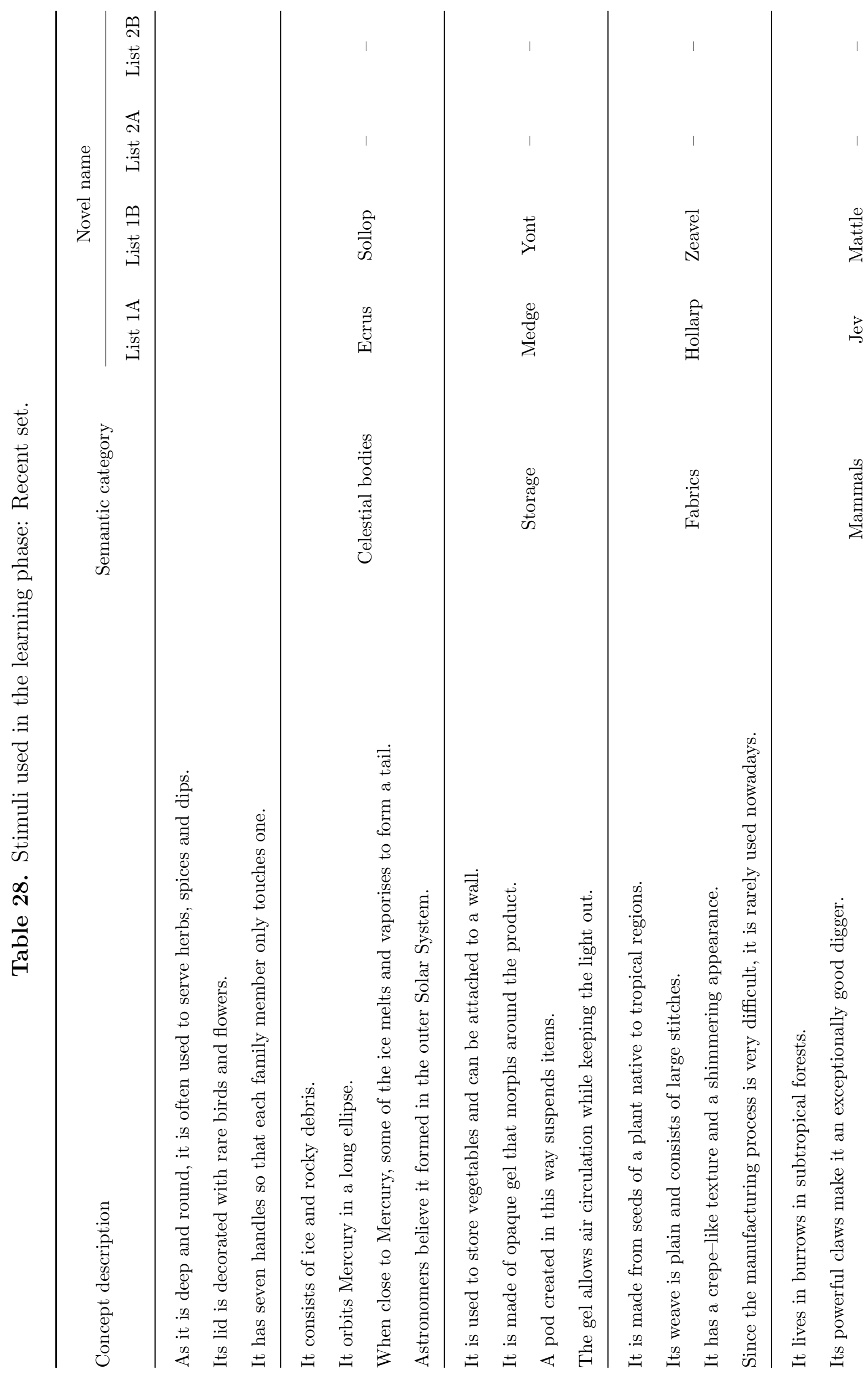




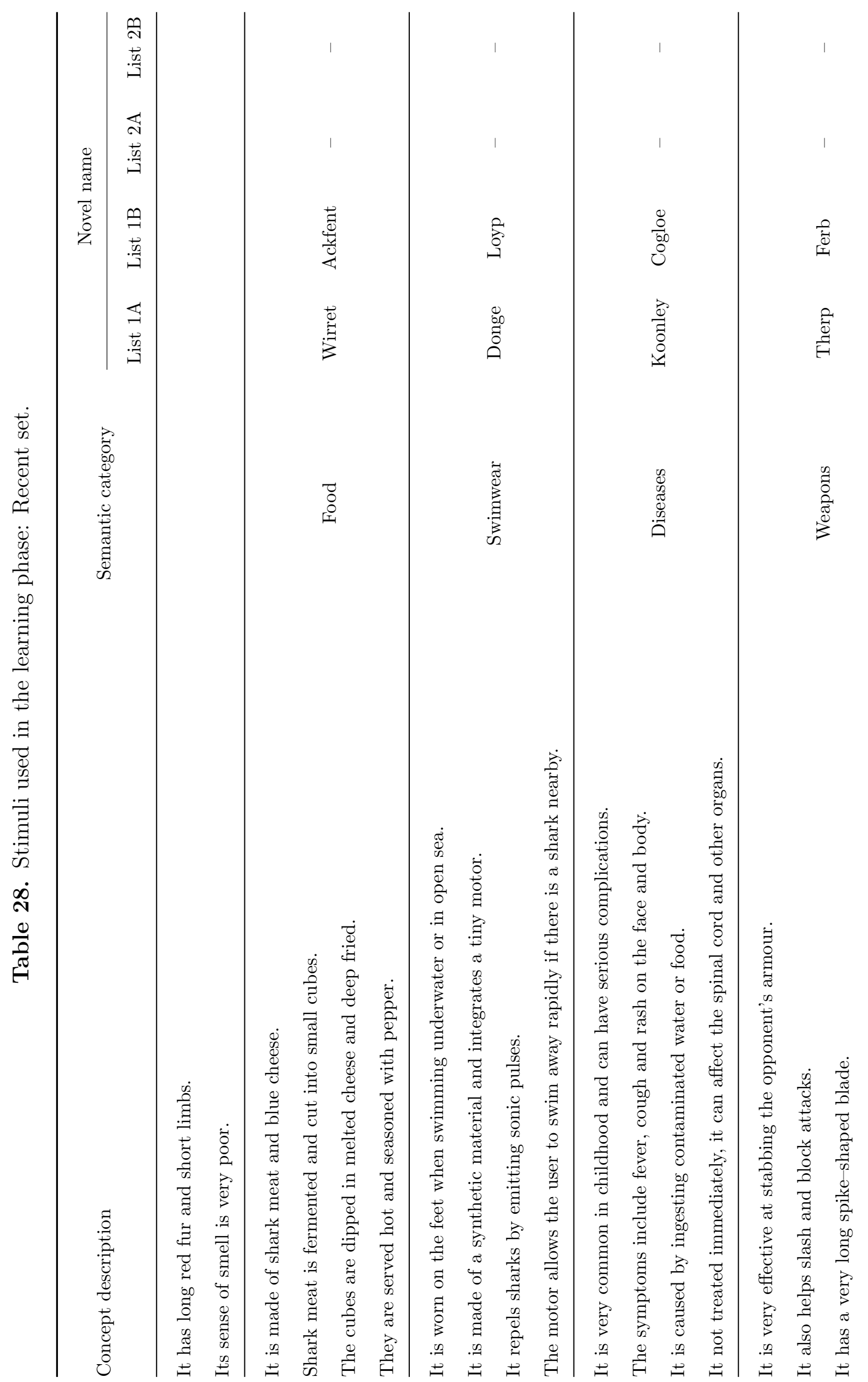




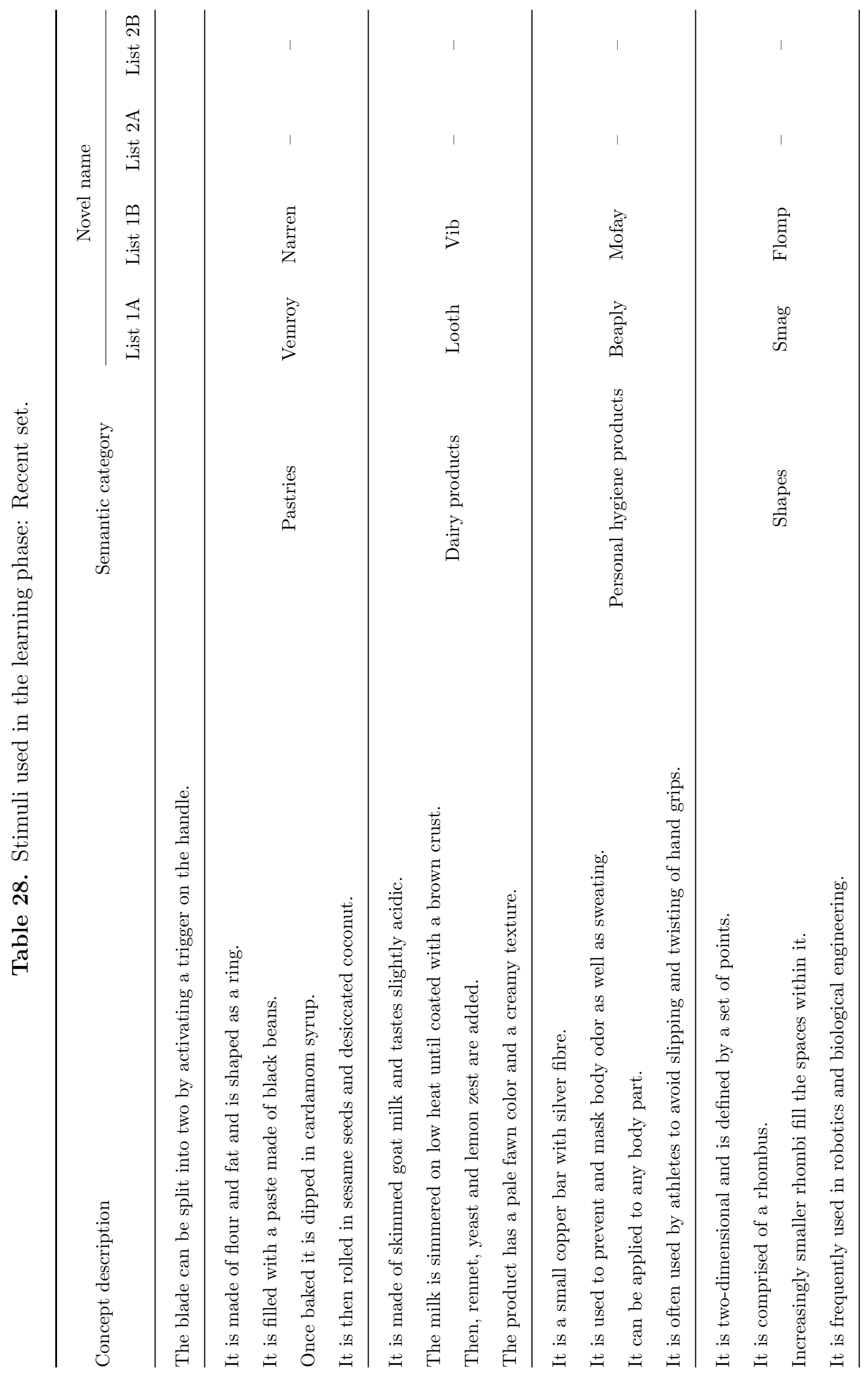




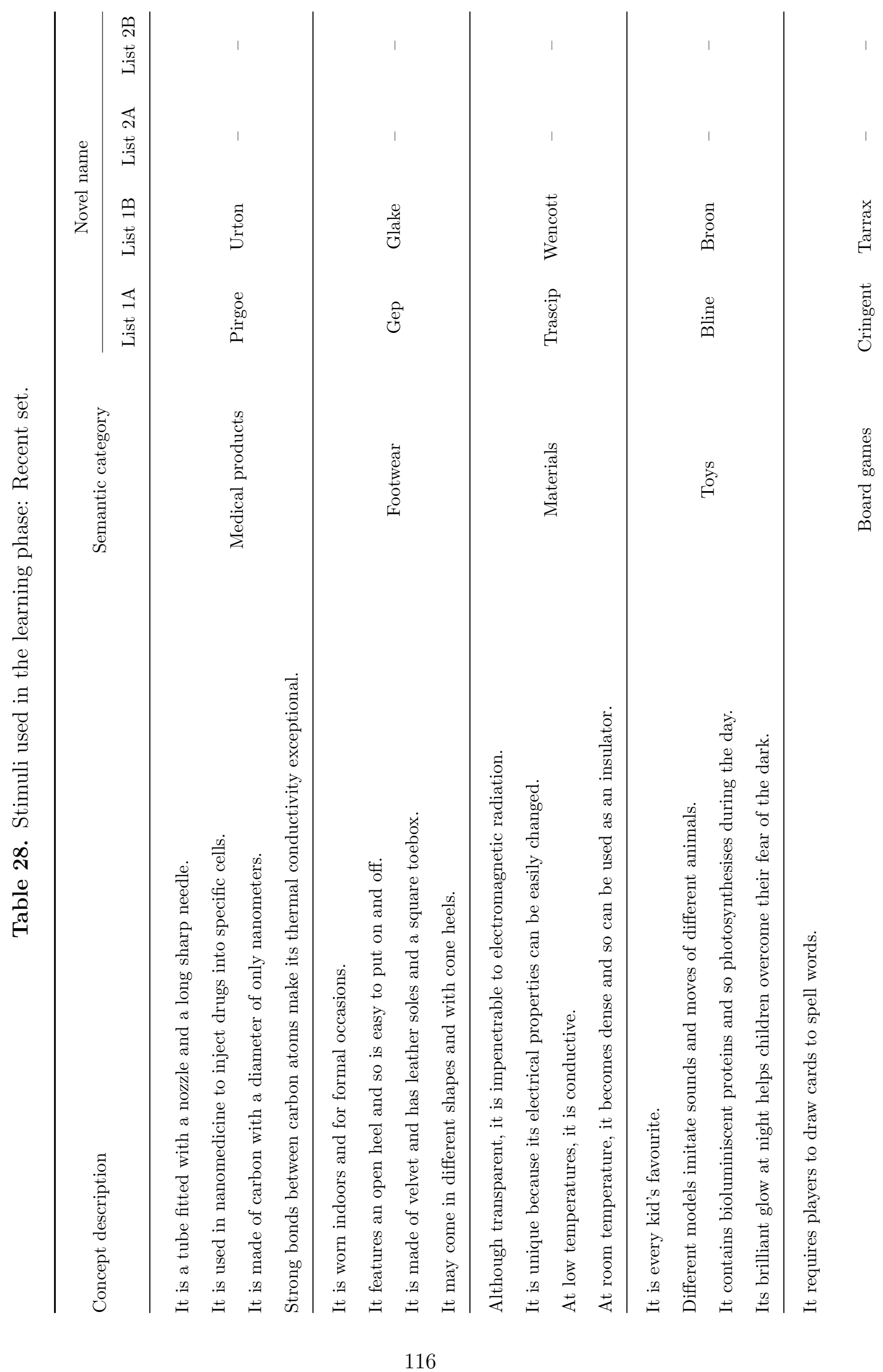




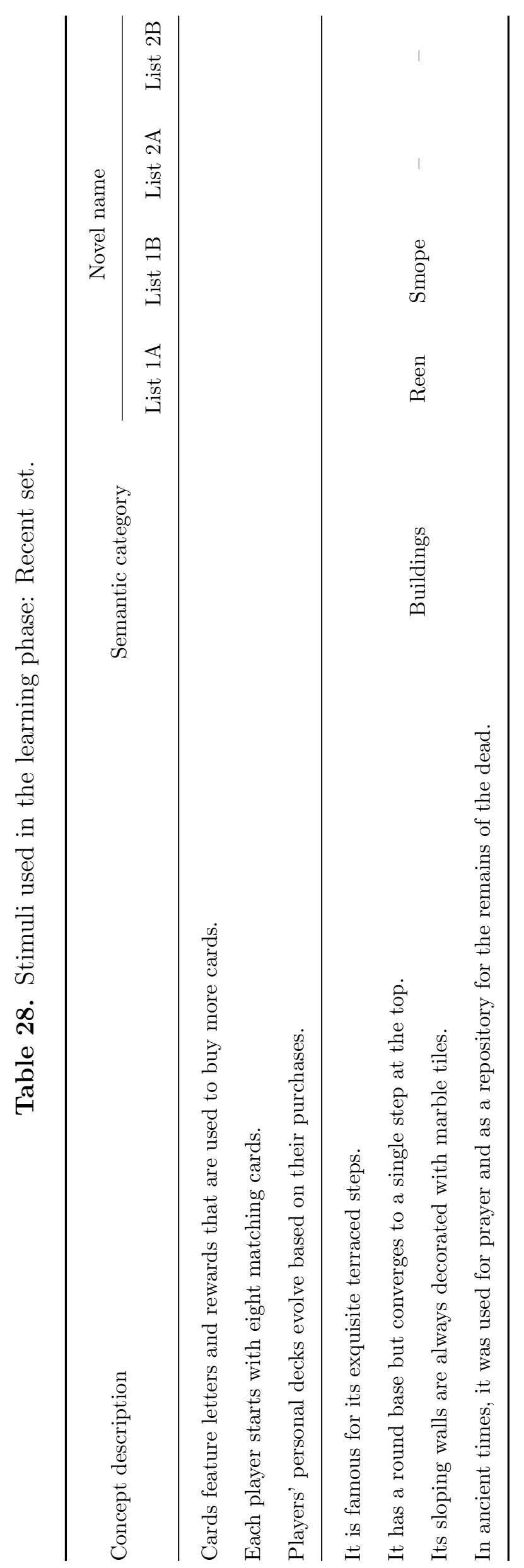




\section{Appendix C Stimuli used in the primed continuous lexical decision task}

\section{C.1 Experimental items}

Note that English pairs used in lists 1A1, 1B1, 2A1 and 2B1 were identical but differed from English pairs used in lists 1A2, 1B2, 2A2 and 2B2 (which, in turn, did not differ from each other).

\section{List 1A1}

Table 29. List 1A1: English pairs.

\begin{tabular}{lccrr}
\hline \multicolumn{2}{c}{ Related } & & \multicolumn{2}{c}{ Unrelated } \\
\cline { 1 - 1 } Prime & Target & & Prime & Target \\
\hline Crow & Magpie & & Tennis & Eucalyptus \\
Hammer & Axe & & Oak & Protractor \\
Salmon & Tuna & & Zucchini & Texta \\
Tuxedo & Jacket & & Juice & Badminton \\
Chair & Bench & Bumblebee & Sapphire \\
Clutch & Bag & & Fedora & Gully \\
Kayak & Dinghy & Canyon & Lemonade \\
Tornado & Hurricane & Pen & Hornet \\
Trumpet & Saxophone & Emerald & Sombrero \\
Tent & Swag & Ruler & Cucumber \\
Pie & Tart & Boardies & Pendant \\
Yogurt & Cream & Polyester & Saucer \\
Soap & Shampoo & Mouse & Star \\
Circle & Ellipse & Planet & Dresser \\
Disinfectant & Antiseptic & Sandwich & Nylon \\
Thongs & Sandals & & Locket & Possum
\end{tabular}




\begin{tabular}{lccr} 
Copper & Bronze & Sword & Burger \\
Doll & Puppet & Plate & Cozzie \\
Backgammon & Chess & Locket & Pneumonia \\
Cathedral & Temple & Influenza & Dagger \\
\hline
\end{tabular}


Table 30. List 1A1: English-novel pairs.

\begin{tabular}{|c|c|c|c|c|}
\hline \multirow{2}{*}{ Target set } & \multicolumn{2}{|c|}{ Related } & \multicolumn{2}{|c|}{ Unrelated } \\
\hline & Prime & Target & Prime & Target \\
\hline \multirow{10}{*}{ Remote } & Cactus & Gimster & Couch & Narren \\
\hline & Scales & Tord & Sinkhole & Vib \\
\hline & Pencil & Sollop & Satchel & Mofay \\
\hline & Soccer & Yont & Cassowary & Flomp \\
\hline & Diamond & Zeavel & Shark & Urton \\
\hline & Ridge & Mattle & Shears & Glake \\
\hline & Cocktail & Ackfent & Piano & Wencott \\
\hline & Butterfly & Loyp & Mat & Broon \\
\hline & Beanie & Cogloe & Helicopter & Tarrax \\
\hline & Radish & Ferb & Overalls & Smope \\
\hline \multirow{10}{*}{ Recent } & Necklace & Affey & Pyramid & Vemroy \\
\hline & Bowl & Flerse & Scrabble & Looth \\
\hline & Comet & Ecrus & Furby & Beaply \\
\hline & Shelf & Medge & Custard & Smag \\
\hline & Cotton & Hollarp & Slipper & Pirgoe \\
\hline & Wombat & Jev & Doughnut & Gep \\
\hline & Fondue & Wirret & Deodorant & Trascip \\
\hline & Flipper & Donge & Glass & Bline \\
\hline & Measles & Koonley & Syringe & Cringent \\
\hline & Bayonet & Therp & Hexagon & Reen \\
\hline
\end{tabular}




\section{List $1 \mathrm{~A} 2$}

Table 31. List 1A2: English pairs.

\begin{tabular}{|c|c|c|c|}
\hline \multicolumn{2}{|c|}{ Related } & \multicolumn{2}{|c|}{ Unrelated } \\
\hline Prime & Target & Prime & Target \\
\hline Oak & Eucalyptus & Chair & Magpie \\
\hline Ruler & Protractor & Tornado & Axe \\
\hline Pen & Texta & Clutch & Tuna \\
\hline Tennis & Badminton & Crow & Jacket \\
\hline Emerald & Sapphire & Salmon & Bench \\
\hline Canyon & Gully & Hammer & Bag \\
\hline Juice & Lemonade & Trumpet & Dinghy \\
\hline Bumblebee & Hornet & Tent & Hurricane \\
\hline Fedora & Sombrero & Kayak & Saxophone \\
\hline Zucchini & Cucumber & Tuxedo & Swag \\
\hline Locket & Pendant & Cathedral & Tart \\
\hline Plate & Saucer & Backgammon & Cream \\
\hline Planet & Star & Doll & Shampoo \\
\hline Wardrobe & Dresser & Yogurt & Ellipse \\
\hline Polyester & Nylon & Thongs & Antiseptic \\
\hline Mouse & Possum & Pie & Sandals \\
\hline Sandwich & Burger & Soap & Bronze \\
\hline Boardies & Cozzie & Copper & Puppet \\
\hline Influenza & Pneumonia & Disinfectant & Chess \\
\hline Sword & Dagger & Circle & Temple \\
\hline
\end{tabular}


Table 32. List 1A2: English-novel pairs.

\begin{tabular}{|c|c|c|c|c|}
\hline \multirow{2}{*}{ Target set } & \multicolumn{2}{|c|}{ Related } & \multicolumn{2}{|c|}{ Unrelated } \\
\hline & Prime & Target & Prime & Target \\
\hline \multirow{10}{*}{ Remote } & Cassowary & Narren & Soccer & Gimster \\
\hline & Shears & Vib & Cactus & Tord \\
\hline & Shark & Mofay & Radish & Sollop \\
\hline & Overalls & Flomp & Cocktail & Yont \\
\hline & Couch & Urton & Butterfly & Zeavel \\
\hline & Satchel & Glake & Beanie & Mattle \\
\hline & Helicopter & Wencott & Ridge & Ackfent \\
\hline & Sinkhole & Broon & Pencil & Loyp \\
\hline & Piano & Tarrax & Diamond & Cogloe \\
\hline & Mat & Smope & Scales & Ferb \\
\hline \multirow{10}{*}{ Recent } & Doughnut & Vemroy & Flipper & Affey \\
\hline & Custard & Looth & Cotton & Flerse \\
\hline & Deodorant & Beaply & Wombat & Ecrus \\
\hline & Hexagon & Smag & Comet & Medge \\
\hline & Syringe & Pirgoe & Fondue & Hollarp \\
\hline & Slipper & Gep & Necklace & Jev \\
\hline & Glass & Trascip & Bayonet & Wirret \\
\hline & Furby & Bline & Bowl & Donge \\
\hline & Scrabble & Cringent & Shelf & Koonley \\
\hline & Pyramid & Reen & Measles & Therp \\
\hline
\end{tabular}




\section{List 1B1}

Table 33. List 1B1: English pairs.

\begin{tabular}{|c|c|c|c|}
\hline \multicolumn{2}{|c|}{ Related } & \multicolumn{2}{|c|}{ Unrelated } \\
\hline Prime & Target & Prime & Target \\
\hline Crow & Magpie & Tennis & Eucalyptus \\
\hline Hammer & Axe & Oak & Protractor \\
\hline Salmon & Tuna & Zucchini & Texta \\
\hline Tuxedo & Jacket & Juice & Badminton \\
\hline Chair & Bench & Bumblebee & Sapphire \\
\hline Clutch & Bag & Fedora & Gully \\
\hline Kayak & Dinghy & Canyon & Lemonade \\
\hline Tornado & Hurricane & Pen & Hornet \\
\hline Trumpet & Saxophone & Emerald & Sombrero \\
\hline Tent & Swag & Ruler & Cucumber \\
\hline Pie & Tart & Boardies & Pendant \\
\hline Yogurt & Cream & Polyester & Saucer \\
\hline Soap & Shampoo & Mouse & Star \\
\hline Circle & Ellipse & Planet & Dresser \\
\hline Disinfectant & Antiseptic & Sandwich & Nylon \\
\hline Thongs & Sandals & Locket & Possum \\
\hline Copper & Bronze & Sword & Burger \\
\hline Doll & Puppet & Plate & Cozzie \\
\hline Backgammon & Chess & Locket & Pneumonia \\
\hline Cathedral & Temple & Influenza & Dagger \\
\hline
\end{tabular}


Table 34. List 1B1: English-novel pairs.

\begin{tabular}{|c|c|c|c|c|}
\hline \multirow{2}{*}{ Target set } & \multicolumn{2}{|c|}{ Related } & \multicolumn{2}{|c|}{ Unrelated } \\
\hline & Prime & Target & Prime & Target \\
\hline \multirow{10}{*}{ Remote } & Cactus & Affey & Couch & Vemroy \\
\hline & Scales & Flerse & Sinkhole & Looth \\
\hline & Pencil & Ecrus & Satchel & Beaply \\
\hline & Soccer & Medge & Cassowary & Smag \\
\hline & Diamond & Hollarp & Shark & Pirgoe \\
\hline & Ridge & Jev & Shears & Gep \\
\hline & Cocktail & Wirret & Piano & Trascip \\
\hline & Butterfly & Donge & Mat & Bline \\
\hline & Beanie & Koonley & Helicopter & Cringent \\
\hline & Radish & Therp & Overalls & Reen \\
\hline \multirow{10}{*}{ Recent } & Necklace & Gimster & Pyramid & Narren \\
\hline & Bowl & Tord & Scrabble & Vib \\
\hline & Comet & Sollop & Furby & Mofay \\
\hline & Shelf & Yont & Custard & Flomp \\
\hline & Cotton & Zeavel & Slipper & Urton \\
\hline & Wombat & Mattle & Doughnut & Glake \\
\hline & Fondue & Ackfent & Deodorant & Wencott \\
\hline & Flipper & Loyp & Glass & Broon \\
\hline & Measles & Cogloe & Syringe & Tarrax \\
\hline & Bayonet & Ferb & Hexagon & Smope \\
\hline
\end{tabular}




\section{List 1B2}

Table 35. List 1B2: English pairs.

\begin{tabular}{|c|c|c|c|}
\hline \multicolumn{2}{|c|}{ Related } & \multicolumn{2}{|c|}{ Unrelated } \\
\hline Prime & Target & Prime & Target \\
\hline Oak & Eucalyptus & Chair & Magpie \\
\hline Ruler & Protractor & Tornado & Axe \\
\hline Pen & Texta & Clutch & Tuna \\
\hline Tennis & Badminton & Crow & Jacket \\
\hline Emerald & Sapphire & Salmon & Bench \\
\hline Canyon & Gully & Hammer & Bag \\
\hline Juice & Lemonade & Trumpet & Dinghy \\
\hline Bumblebee & Hornet & Tent & Hurricane \\
\hline Fedora & Sombrero & Kayak & Saxophone \\
\hline Zucchini & Cucumber & Tuxedo & Swag \\
\hline Locket & Pendant & Cathedral & Tart \\
\hline Plate & Saucer & Backgammon & Cream \\
\hline Planet & Star & Doll & Shampoo \\
\hline Wardrobe & Dresser & Yogurt & Ellipse \\
\hline Polyester & Nylon & Thongs & Antiseptic \\
\hline Mouse & Possum & Pie & Sandals \\
\hline Sandwich & Burger & Soap & Bronze \\
\hline Boardies & Cozzie & Copper & Puppet \\
\hline Influenza & Pneumonia & Disinfectant & Chess \\
\hline Sword & Dagger & Circle & Temple \\
\hline
\end{tabular}


Table 36. List 1B2: English-novel pairs.

\begin{tabular}{|c|c|c|c|c|}
\hline \multirow{2}{*}{ Target set } & \multicolumn{2}{|c|}{ Related } & \multicolumn{2}{|c|}{ Unrelated } \\
\hline & Prime & Target & Prime & Target \\
\hline \multirow{10}{*}{ Remote } & Cassowary & Vemroy & Soccer & Affey \\
\hline & Shears & Looth & Cactus & Flerse \\
\hline & Shark & Beaply & Radish & Ecrus \\
\hline & Overalls & Smag & Cocktail & Medge \\
\hline & Couch & Pirgoe & Butterfly & Hollarp \\
\hline & Satchel & Gep & Beanie & Jev \\
\hline & Helicopter & Trascip & Ridge & Wirret \\
\hline & Sinkhole & Bline & Pencil & Donge \\
\hline & Piano & Cringent & Diamond & Koonley \\
\hline & Mat & Reen & Scales & Therp \\
\hline \multirow{10}{*}{ Recent } & Doughnut & Narren & Flipper & Gimster \\
\hline & Custard & Vib & Cotton & Tord \\
\hline & Deodorant & Mofay & Wombat & Sollop \\
\hline & Hexagon & Flomp & Comet & Yont \\
\hline & Syringe & Urton & Fondue & Zeavel \\
\hline & Slipper & Glake & Necklace & Mattle \\
\hline & Glass & Wencott & Bayonet & Ackfent \\
\hline & Furby & Broon & Bowl & Loyp \\
\hline & Scrabble & Tarrax & Shelf & Cogloe \\
\hline & Pyramid & Smope & Measles & Ferb \\
\hline
\end{tabular}




\section{List 2A1}

Table 37. List 2A1: English pairs.

\begin{tabular}{|c|c|c|c|}
\hline \multicolumn{2}{|c|}{ Related } & \multicolumn{2}{|c|}{ Unrelated } \\
\hline Prime & Target & Prime & Target \\
\hline Crow & Magpie & Tennis & Eucalyptus \\
\hline Hammer & Axe & Oak & Protractor \\
\hline Salmon & Tuna & Zucchini & Texta \\
\hline Tuxedo & Jacket & Juice & Badminton \\
\hline Chair & Bench & Bumblebee & Sapphire \\
\hline Clutch & $\mathrm{Bag}$ & Fedora & Gully \\
\hline Kayak & Dinghy & Canyon & Lemonade \\
\hline Tornado & Hurricane & Pen & Hornet \\
\hline Trumpet & Saxophone & Emerald & Sombrero \\
\hline Tent & Swag & Ruler & Cucumber \\
\hline Pie & Tart & Boardies & Pendant \\
\hline Yogurt & Cream & Polyester & Saucer \\
\hline Soap & Shampoo & Mouse & Star \\
\hline Circle & Ellipse & Planet & Dresser \\
\hline Disinfectant & Antiseptic & Sandwich & Nylon \\
\hline Thongs & Sandals & Locket & Possum \\
\hline Copper & Bronze & Sword & Burger \\
\hline Doll & Puppet & Plate & Cozzie \\
\hline Backgammon & Chess & Locket & Pneumonia \\
\hline Cathedral & Temple & Influenza & Dagger \\
\hline
\end{tabular}


Table 38. List 2A1: English-novel pairs.

\begin{tabular}{|c|c|c|c|c|}
\hline \multirow{2}{*}{ Target set } & \multicolumn{2}{|c|}{ Related } & \multicolumn{2}{|c|}{ Unrelated } \\
\hline & Prime & Target & Prime & Target \\
\hline \multirow{10}{*}{ Remote } & Necklace & Gimster & Pyramid & Narren \\
\hline & Bowl & Tord & Scrabble & Vib \\
\hline & Comet & Sollop & Furby & Mofay \\
\hline & Shelf & Yont & Custard & Flomp \\
\hline & Cotton & Zeavel & Slipper & Urton \\
\hline & Wombat & Mattle & Doughnut & Glake \\
\hline & Fondue & Ackfent & Deodorant & Wencott \\
\hline & Flipper & Loyp & Glass & Broon \\
\hline & Measles & Cogloe & Syringe & Tarrax \\
\hline & Bayonet & Ferb & Hexagon & Smope \\
\hline \multirow{10}{*}{ Recent } & Cactus & Affey & Couch & Vemroy \\
\hline & Scales & Flerse & Sinkhole & Looth \\
\hline & Pencil & Ecrus & Satchel & Beaply \\
\hline & Soccer & Medge & Cassowary & Smag \\
\hline & Diamond & Hollarp & Shark & Pirgoe \\
\hline & Ridge & Jev & Shears & Gep \\
\hline & Cocktail & Wirret & Piano & Trascip \\
\hline & Butterfly & Donge & Mat & Bline \\
\hline & Beanie & Koonley & Helicopter & Cringent \\
\hline & Radish & Therp & Overalls & Reen \\
\hline
\end{tabular}




\section{List $2 \mathrm{~A} 2$}

Table 39. List 2A2: English pairs.

\begin{tabular}{|c|c|c|c|}
\hline \multicolumn{2}{|c|}{ Related } & \multicolumn{2}{|c|}{ Unrelated } \\
\hline Prime & Target & Prime & Target \\
\hline Oak & Eucalyptus & Chair & Magpie \\
\hline Ruler & Protractor & Tornado & Axe \\
\hline Pen & Texta & Clutch & Tuna \\
\hline Tennis & Badminton & Crow & Jacket \\
\hline Emerald & Sapphire & Salmon & Bench \\
\hline Canyon & Gully & Hammer & Bag \\
\hline Juice & Lemonade & Trumpet & Dinghy \\
\hline Bumblebee & Hornet & Tent & Hurricane \\
\hline Fedora & Sombrero & Kayak & Saxophone \\
\hline Zucchini & Cucumber & Tuxedo & Swag \\
\hline Locket & Pendant & Cathedral & Tart \\
\hline Plate & Saucer & Backgammon & Cream \\
\hline Planet & Star & Doll & Shampoo \\
\hline Wardrobe & Dresser & Yogurt & Ellipse \\
\hline Polyester & Nylon & Thongs & Antiseptic \\
\hline Mouse & Possum & Pie & Sandals \\
\hline Sandwich & Burger & Soap & Bronze \\
\hline Boardies & Cozzie & Copper & Puppet \\
\hline Influenza & Pneumonia & Disinfectant & Chess \\
\hline Sword & Dagger & Circle & Temple \\
\hline
\end{tabular}


Table 40. List 2A2: English-novel pairs.

\begin{tabular}{|c|c|c|c|c|}
\hline \multirow{2}{*}{ Target set } & \multicolumn{2}{|c|}{ Related } & \multicolumn{2}{|c|}{ Unrelated } \\
\hline & Prime & Target & Prime & Target \\
\hline \multirow{10}{*}{ Remote } & Doughnut & Narren & Flipper & Gimster \\
\hline & Custard & Vib & Cotton & Tord \\
\hline & Deodorant & Mofay & Wombat & Sollop \\
\hline & Hexagon & Flomp & Comet & Yont \\
\hline & Syringe & Urton & Fondue & Zeavel \\
\hline & Slipper & Glake & Necklace & Mattle \\
\hline & Glass & Wencott & Bayonet & Ackfent \\
\hline & Furby & Broon & Bowl & Loyp \\
\hline & Scrabble & Tarrax & Shelf & Cogloe \\
\hline & Pyramid & Smope & Measles & Ferb \\
\hline \multirow{10}{*}{ Recent } & Cassowary & Vemroy & Soccer & Affey \\
\hline & Shears & Looth & Cactus & Flerse \\
\hline & Shark & Beaply & Radish & Ecrus \\
\hline & Overalls & Smag & Cocktail & Medge \\
\hline & Couch & Pirgoe & Butterfly & Hollarp \\
\hline & Satchel & Gep & Beanie & Jev \\
\hline & Helicopter & Trascip & Ridge & Wirret \\
\hline & Sinkhole & Bline & Pencil & Donge \\
\hline & Piano & Cringent & Diamond & Koonley \\
\hline & Mat & Reen & Scales & Therp \\
\hline
\end{tabular}




\section{List 2B1}

Table 41. List 2B1: English pairs.

\begin{tabular}{|c|c|c|c|}
\hline \multicolumn{2}{|c|}{ Related } & \multicolumn{2}{|c|}{ Unrelated } \\
\hline Prime & Target & Prime & Target \\
\hline Crow & Magpie & Tennis & Eucalyptus \\
\hline Hammer & Axe & Oak & Protractor \\
\hline Salmon & Tuna & Zucchini & Texta \\
\hline Tuxedo & Jacket & Juice & Badminton \\
\hline Chair & Bench & Bumblebee & Sapphire \\
\hline Clutch & $\mathrm{Bag}$ & Fedora & Gully \\
\hline Kayak & Dinghy & Canyon & Lemonade \\
\hline Tornado & Hurricane & Pen & Hornet \\
\hline Trumpet & Saxophone & Emerald & Sombrero \\
\hline Tent & Swag & Ruler & Cucumber \\
\hline Pie & Tart & Boardies & Pendant \\
\hline Yogurt & Cream & Polyester & Saucer \\
\hline Soap & Shampoo & Mouse & Star \\
\hline Circle & Ellipse & Planet & Dresser \\
\hline Disinfectant & Antiseptic & Sandwich & Nylon \\
\hline Thongs & Sandals & Locket & Possum \\
\hline Copper & Bronze & Sword & Burger \\
\hline Doll & Puppet & Plate & Cozzie \\
\hline Backgammon & Chess & Locket & Pneumonia \\
\hline Cathedral & Temple & Influenza & Dagger \\
\hline
\end{tabular}


Table 42. List 2B1: English-novel pairs.

\begin{tabular}{|c|c|c|c|c|}
\hline \multirow{2}{*}{ Target set } & \multicolumn{2}{|c|}{ Related } & \multicolumn{2}{|c|}{ Unrelated } \\
\hline & Prime & Target & Prime & Target \\
\hline \multirow{10}{*}{ Remote } & Necklace & Affey & Pyramid & Vemroy \\
\hline & Bowl & Flerse & Scrabble & Looth \\
\hline & Comet & Ecrus & Furby & Beaply \\
\hline & Shelf & Medge & Custard & Smag \\
\hline & Cotton & Hollarp & Slipper & Pirgoe \\
\hline & Wombat & Jev & Doughnut & Gep \\
\hline & Fondue & Wirret & Deodorant & Trascip \\
\hline & Flipper & Donge & Glass & Bline \\
\hline & Measles & Koonley & Syringe & Cringent \\
\hline & Bayonet & Therp & Hexagon & Reen \\
\hline \multirow{10}{*}{ Recent } & Cactus & Gimster & Couch & Narren \\
\hline & Scales & Tord & Sinkhole & Vib \\
\hline & Pencil & Sollop & Satchel & Mofay \\
\hline & Soccer & Yont & Cassowary & Flomp \\
\hline & Diamond & Zeavel & Shark & Urton \\
\hline & Ridge & Mattle & Shears & Glake \\
\hline & Cocktail & Ackfent & Piano & Wencott \\
\hline & Butterfly & Loyp & Mat & Broon \\
\hline & Beanie & Cogloe & Helicopter & Tarrax \\
\hline & Radish & Ferb & Overalls & Smope \\
\hline
\end{tabular}




\section{List 2B2}

Table 43. List 2B2: English pairs.

\begin{tabular}{|c|c|c|c|}
\hline \multicolumn{2}{|c|}{ Related } & \multicolumn{2}{|c|}{ Unrelated } \\
\hline Prime & Target & Prime & Target \\
\hline Oak & Eucalyptus & Chair & Magpie \\
\hline Ruler & Protractor & Tornado & Axe \\
\hline Pen & Texta & Clutch & Tuna \\
\hline Tennis & Badminton & Crow & Jacket \\
\hline Emerald & Sapphire & Salmon & Bench \\
\hline Canyon & Gully & Hammer & Bag \\
\hline Juice & Lemonade & Trumpet & Dinghy \\
\hline Bumblebee & Hornet & Tent & Hurricane \\
\hline Fedora & Sombrero & Kayak & Saxophone \\
\hline Zucchini & Cucumber & Tuxedo & Swag \\
\hline Locket & Pendant & Cathedral & Tart \\
\hline Plate & Saucer & Backgammon & Cream \\
\hline Planet & Star & Doll & Shampoo \\
\hline Wardrobe & Dresser & Yogurt & Ellipse \\
\hline Polyester & Nylon & Thongs & Antiseptic \\
\hline Mouse & Possum & Pie & Sandals \\
\hline Sandwich & Burger & Soap & Bronze \\
\hline Boardies & Cozzie & Copper & Puppet \\
\hline Influenza & Pneumonia & Disinfectant & Chess \\
\hline Sword & Dagger & Circle & Temple \\
\hline
\end{tabular}


Table 44. List 2B2: English-novel pairs.

\begin{tabular}{|c|c|c|c|c|}
\hline \multirow{2}{*}{ Target set } & \multicolumn{2}{|c|}{ Related } & \multicolumn{2}{|c|}{ Unrelated } \\
\hline & Prime & Target & Prime & Target \\
\hline \multirow{10}{*}{ Remote } & Doughnut & Vemroy & Flipper & Affey \\
\hline & Custard & Looth & Cotton & Flerse \\
\hline & Deodorant & Beaply & Wombat & Ecrus \\
\hline & Hexagon & Smag & Comet & Medge \\
\hline & Syringe & Pirgoe & Fondue & Hollarp \\
\hline & Slipper & Gep & Necklace & Jev \\
\hline & Glass & Trascip & Bayonet & Wirret \\
\hline & Furby & Bline & Bowl & Donge \\
\hline & Scrabble & Cringent & Shelf & Koonley \\
\hline & Pyramid & Reen & Measles & Therp \\
\hline \multirow{10}{*}{ Recent } & Cassowary & Narren & Soccer & Gimster \\
\hline & Shears & Vib & Cactus & Tord \\
\hline & Shark & Mofay & Radish & Sollop \\
\hline & Overalls & Flomp & Cocktail & Yont \\
\hline & Couch & Urton & Butterfly & Zeavel \\
\hline & Satchel & Glake & Beanie & Mattle \\
\hline & Helicopter & Wencott & Ridge & Ackfent \\
\hline & Sinkhole & Broon & Pencil & Loyp \\
\hline & Piano & Tarrax & Diamond & Cogloe \\
\hline & Mat & Smope & Scales & Ferb \\
\hline
\end{tabular}




\section{C.2 Filler items}

Note that filler items were identical across all eight lists.

Table 45. Filler items.

\begin{tabular}{|c|c|c|c|c|}
\hline Nall & Dreace & Limpry & Blit & Eashoy \\
\hline Wap & Tolb & Lannot & Thesk & Mafet \\
\hline Thelm & Theel & Floster & Tem & Jievong \\
\hline Freech & Roff & Fossay & Skoath & Tellaw \\
\hline Baff & Gorp & Braistler & Tanvek & Broyress \\
\hline Soud & Hace & Bollay & Avift & Faynick \\
\hline Lenge & Moobs & Deret & Jantsy & Fimbley \\
\hline Pristle & Bloyce & Gronner & Ealpet & Brepster \\
\hline Ayne & Ayket & Suppix & Jeedney & Darvel \\
\hline Dwud & Jeyfene & Plafaid & Dallot & Larset \\
\hline Crought & Emplim & Reywee & Baspert & Geabler \\
\hline Jish & Zenquel & Cengore & Harrel & Ristem \\
\hline Twip & Larpell & Vaggy & Timloe & Nottle \\
\hline Troot & Dandow & Noalak & Veroe & Zalph \\
\hline Therk & Ferub & Talmit & Kawdy & Tholve \\
\hline Glab & Fanser & Tornuke & Phebune & Frem \\
\hline Gruke & Blikeet & Bant & Trofime & Fove \\
\hline Meef & Breamol & Molf & Cedoak & Spee \\
\hline Benth & Maffrebe & Gith & Ployner & Larce \\
\hline Prup & Geereh & Zash & Sheaser & Polx \\
\hline Nisp & Shurvoke & Chob & Flarny & Plap \\
\hline Zek & Quenore & Woip & Nelber & Debe \\
\hline Thodd & Roathel & Yeese & Belfy & Ulph \\
\hline Fop & Calmong & Grolve & Loring & Cang \\
\hline Fath & Consvot & Plyth & Quiplee & Chig \\
\hline Sprean & Norey & Pelf & Tebop & Tilk \\
\hline Shrowl & Tremish & Lig & Croiler & Thrume \\
\hline
\end{tabular}




\begin{tabular}{lcccr} 
Pleep & Thournant & Floop & Oonie & Rolt \\
Pluce & Eyleme & Shug & Emrowse & Ghonge \\
Lirge & Jievers & Nug & Nithoar & Vead \\
Erdse & Ancoosh & Pangle & Boltem & Boak \\
Chasp & Inrant & Thog & Sythlom & Milp \\
\hline
\end{tabular}




\section{Appendix D Bayes factors for lexicality models containing different contrasts (with different priors)}

Table 46. Bayes factors for lexicality models containing different contrasts (with different priors). $\quad M_{1}$ denotes the numerator model and $M_{2}$ the denominator model. NonEng stands for the contrast between the nonwords and the English words, RecNon stands for the contrast between the recent novel names and the nonwords, RemRec stands for the contrast between the remote and the recent novel names, and Null stands for the null model (no fixed effects).

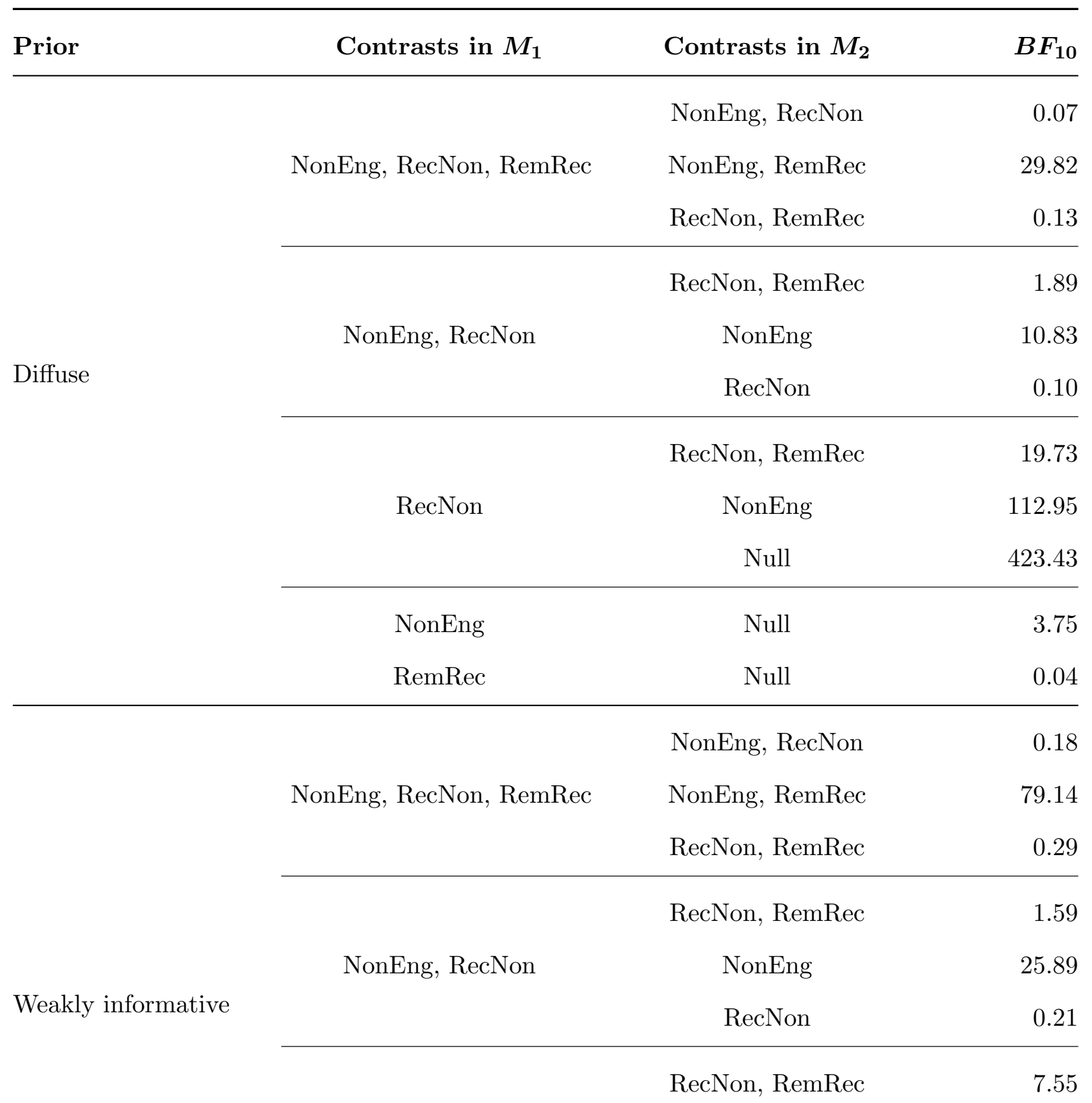




\begin{tabular}{|c|c|c|c|}
\hline & & NonEng & 123.13 \\
\hline & & Null & 846.21 \\
\hline & NonEng & Null & 6.87 \\
\hline & RemRec & Null & 0.09 \\
\hline & & NonEng, RecNon & 0.29 \\
\hline & NonEng, RecNon, RemRec & NonEng, RemRec & 171.53 \\
\hline & & RecNon, RemRec & 0.45 \\
\hline & & RecNon, RemRec & 1.55 \\
\hline & NonEng, RecNon & NonEng & 77.04 \\
\hline Strongly informative & & RecNon & 0.58 \\
\hline & & RecNon, RemRec & 2.67 \\
\hline & RecNon & NonEng & 133.10 \\
\hline & & Null & 1046.45 \\
\hline & NonEng & Null & 7.86 \\
\hline & RemRec & Null & 0.10 \\
\hline
\end{tabular}




\section{References}

Addante, R. J., Ranganath, C., \& Yonelinas, A. P. (2012). Examining ERP correlates of recognition memory: Evidence of accurate source recognition without recollection. NeuroImage, 62, 439-450. https://doi.org/10.1016/j.neuroimage.2012.04.031

Amsel, B. D., Urbach, T. P., \& Kutas, M. (2013). Alive and grasping: Stable and rapid semantic access to an object category but not object graspability. NeuroImage, 77, 1-13. https://doi.org/10.1016/j.neuroimage.2013.03.058

Anglin, J. M. (2000). Vocabulary Development: A Morphological Analysis. Wiley.

Baayen, R. (2008). Analyzing Linguistic Data. A Practical Introduction to Statistics Using R. Cambridge University Press.

Bakker, I., Takashima, A., van Hell, J. G., Janzen, G., \& McQueen, J. M. (2014). Competition from unseen or unheard novel words: Lexical consolidation across modalities. Journal of Memory and Language, 73, 116-130. http://dx.doi.org/10.1016/j.jml.2014.03.002

Bakker, I., Takashima, A., van Hell, J. G., Janzen, G., \& McQueen, J. M. (2015). Tracking lexical consolidation with ERPs: Lexical and semantic-priming effects on N400 and LPC responses to newly-learned words. Neuropsychologia, 79, 33-41. https://doi.org/10.1016/j.neuropsychologia.2015.10.020

Balass, M., Nelson, J. R., \& Perfetti, C. A. (2010). Word learning: An ERP investigation of word experience effects on recognition and word processing. Contemporary Educational Psychology, 35, 126-140. https://doi.org/10.1016/j.cedpsych.2010.04.001

Barr, D. J., Levy, R., Scheepers, C., \& Tily, H. J. (2013). Random effects structure for confirmatory hypothesis testing: Keep it maximal. Journal of Memory and Language, 68(3), 255-278. https://doi.org/10.1016/j.jml.2012.11.001

Batterink, L., \& Neville, H. (2011). Implicit and explicit mechanisms of word learning in a narrative context: An event-related potential study. Journal of Cognitive Neuroscience, 23, 3181-3196. https://doi.org/10.1162/jocn_a_00013

Bellec, P., Lavoie-Courchesne, S., Dickinson, P., Lerch, J., Zijdenbos, A., \& Evans, A. (2012). The pipeline system for Octave and Matlab (PSOM): A lightweight scripting framework and execution engine for scientific workflows. Frontiers in Neuroinformatics, 6(7). https://doi.org/10.3389/fninf.2012.00007

Bennett, C. H. (1976). Efficient estimation of free energy differences from Monte Carlo data. Journal of Computational Physics, 22(2), 245-268. https://doi.org/10.1016/0021-9991(76)90078-4

Bentin, S. (1987). Event-related potentials, semantic processes, and expectancy factors in word recognition. Brain and Language, 31(2), 308-327. https://doi.org/10.1016/0093-934X(87)90077-0

Bentin, S., McCarthy, G., \& Wood, C. C. (1985). Event-related potentials, lexical decision, and semantic priming. Electroencephalography and Clinical Neurophysiology, 60 (4), 343-355. https://doi.org/10.1016/0013-4694(85)90008-2

Bigdely-Shamlo, N., Mullen, T., Kothe, C., Su, K., \& Robbins, K. A. (2015). The PREP pipeline: Standardised preprocessing for large-scale EEG analysis. Frontiers in Neuroinformatics, 9(16). https://doi.org/10.3389/fninf.2015.00016

Bjork, R., \& Kroll, J. (2015). Desirable difficulties in vocabulary learning. American Journal of Psychology, 128, 241-252. https://doi.org/10.5406/amerjpsyc.128.2.0241 
Boersma, P., \& Weenink, D. (2014). Praat: Doing phonetics by computer (Version 6.0.57). https://www.fon.hum.uva.nl/praat/

Borovsky, A., Elman, J. L., \& Kutas, M. (2012). Once is enough: N400 indexes semantic integration of novel word meanings from a single exposure in context. Language Learning and Development, 8(3), 278-302. https://doi.org/10.1080/15475441.2011.614893

Borovsky, A., Kutas, M., \& Elman, J. (2010). Learning to use words: Event-related potentials index single-shot contextual word learning. Cognition, 116(2), 289-296. https://doi.org/10.1016/j.cognition.2010.05.004

Borovsky, A., Kutas, M., \& Elman, J. (2013). Getting it right: Word learning across the hemispheres. Neuropsychologia, 51(5), 825-837. https://doi.org/10.1016/j.neuropsychologia.2013.01.027

Bowers, J., Davis, C., \& Hanley, D. (2005). Interfering neighbours: The impact of novel word learning on the identification of visually similar words. Cognition, 97, B45-B54. https://doi.org/10.1016/j.cognition.2005.02.002

Breitenstein, C., Zwitserlood, P., de Vries, M. H., Feldhues, C., Knecht, S., \& Dobel, C. (2007). Five days versus a lifetime: Intense associative vocabulary training generates lexically integrated words. Restorative Neurology and Neuroscience, 25, 493-500.

Brouwer, H., Crocker, M. W., Venhuizen, N. J., \& Hoeks, J. C. J. (2017). A neurocomputational model of the N400 and the P600 in language processing. Cognitive Science, 41(S6), 1318-1352. https://doi.org/10.1111/cogs.12461

Brouwer, H., Fitz, H., \& Hoeks, J. (2012). Getting real about Semantic illusions: Rethinking the functional role of the P600 in language comprehension. Brain Research, 1446, 127-143. https://doi.org/10.1016/j.brainres.2012.01.055

Brown, C., \& Hagoort, P. (1993). The processing nature of the N400: Evidence from masked priming. Journal of Cognitive Neuroscience, 5(1), 34-44. https://doi.org/10.1162/jocn.1993.5.1.34

Brysbaert, M., Stevens, M., Mandera, P., \& Keuleers, E. (2016). How many words do we know? Practical estimates of vocabulary size dependent on word definition, the degree of language input and the participant's age. Frontiers in Psychology, 7, 1116. https://doi.org/10.3389/fpsyg.2016.01116

Bürkner, P. (2017a). Advanced Bayesian multilevel modeling with the $R$ package brms. arXiv:1705.11123

Bürkner, P. (2017b). brms: An R package for Bayesian multilevel models using Stan. Journal of Statistical Software, 80(1), 1-28. https://doi:10.18637/jss.v080.i01

Cansino, S., \& Trejo-Morales, P. (2008). Neurophysiology of successful encoding and retrieval of source memory. Cognitive, Affective, 8 Behavioral Neuroscience, 80(1), 85-98. https://doi.org/10.3758/cabn.8.1.85

Cepeda, N. J., Pashler, H., Vul, E., Wixted, J. T., \& Rohrer, D. (2006). Distributed practice in verbal recall tasks: A review and quantitative synthesis. Psychological Bulletin, 132, 354-380. https://doi.org/10.1037/0033-2909.132.3.354

Chaumon, M., Bishop, D. V., \& Busch, N. A. (2015). A practical guide to the selection of independent components of the electroencephalogram for artifact correction. Journal of Neuroscience Methods, 250, 47-63. https://doi.org/10.1016/j.jneumeth.2015.02.025

Cheimariou, A., Farmer, T. A., \& Gordon, J. K. (2018). Lexical prediction in the aging brain: The effects of predictiveness and congruency on the N400 ERP component. Aging, Neuropsychology, and Cognition, 26(5), 781-806. 
https://doi.org/10.1080/13825585.2018.1529733

Chwilla, D. J., Brown, C. M., \& Hagoort, P. (1995). The N400 as a function of the level of processing. Psychophysiology, 32(3), 274-285. https://doi.org/10.1111/j.14698986.1995.tb02956.x

Chwilla, D. J., Kolk, H. H. J., \& Mulder, G. (2000). Mediated priming in the lexical decision task: Evidence from event-related potentials and reaction time. Journal of Memory and Language, 42(3), 314-341. https://doi.org/10.1006/jmla.1999.2680

Clay, F., Bowers, J., Davis, C., \& Hanley, D. (2007). Teaching adults new words: The role of practice and consolidation. Journal of Experimental Psychology: Learning, Memory, and Cognition, 33, 970-976. https://doi.org/10.1037/0278-7393.33.5.970

Collins, A. M., \& Loftus, E. F. (1975). A spreading-activation theory of semantic processing. Psychological Review, 82(6), 407-428. https://doi.org/10.1037/0033295X.82.6.407

Curran, T. (1999). The electrophysiology of incidental and intentional retrieval: ERP old/new effects in lexical decision and recognition memory. Neuropsychologia, 37(7), 771-785. https://doi.org/10.1016/S0028-3932(98)00133-X

Davachi, L., \& Dobbins, I. G. (2008). Declarative memory. Current Directions in Psychological Science, 17(2), 112-118. https://doi.org/10.1111/j.14678721.2008.00559.x

Davis, M. H., Di Betta, A. M., Macdonald, M. J., \& Gaskell, M. G. (2009). Learning and consolidation of novel spoken words. Journal of Cognitive Neuroscience, 21, 803-820. https://doi.org/10.1162/jocn.2009.21059

Davis, M. H., \& Gaskell, M. G. (2009). A complementary systems account of word learning: Neural and behavioural evidence. Philosophical Transactions of the Royal Society B: Biological Sciences, 364, 3773-3800. https://doi.org/10.1098/rstb.2009.0111

Deacon, D., Grose-Fifer, J., Yang, C. M., Stanick, V., Hewitt, S., \& Dynowska, A. (2004). Evidence for a new conceptualization of semantic representation in the left and right cerebral hemispheres. Cortex, 40(3), 467-478. https://doi.org/10.1016/S00109452(08)70140-0

De Deyne, S., Navarro, D. J., Perfors, A., Brysbaert, M., \& Storms, G. (2018). The 'Small World of Words' English word association norms for over 12000 cue words. Behavior Research Methods, 51, 987-1006. https://doi.org/10.3758/s13428-018-1115-7

Dehaene, S., Naccache, L., Cohen, L., Le Bihan, D., Mangin, J., Poline, J., \& Rivière, D. (2001). Cerebral mechanisms of word masking and unconscious repetition priming. Nature Neuroscience, 4, 752-758. https://doi.org/10.1038/89551

Delogu, F., Brouwer, H., \& Crocker, M. W. (2019). Event-related potentials index lexical retrieval (N400) and integration (P600) during language comprehension. Brain and Cognition, 135, 103569. https://doi.org/10.1016/j.bandc.2019.05.007

Delorme, A., \& Makeig, S. (2014). EEGLAB: An open source toolbox for analysis of single-trial EEG dynamics including independent component analysis. Journal of Neuroscience Methods, 134(1), 9-21. https://10.1016/j.jneumeth.2003.10.009

de Mornay Davies, P. (1998). Automatic semantic priming: The contribution of lexicaland semantic-level processes. European Journal of Cognitive Psychology, 10(4), 389-412. https://doi.org/10.1080/713752286

De Santis, F., \& Gubbiotti, S. (2021). Sample size requirements for calibrated approximate credible intervals for proportions in clinical trials. International Journal of Environmental Research and Public Health, 18, 595. 
https://doi.org/10.3390/ijerph18020595

de Wit, B., \& Kinoshita, S. (2015). The masked semantic priming effect is task dependent: Reconsidering the automatic spreading activation process. Journal of Experimental Psychology: Learning, Memory, and Cognition, 41(4), 1062-1075. https://doi.org/10.1037/xlm0000074

Diana, R. A., Yonelinas, A. P., \& Ranganath, C. (2007). Imaging recollection and familiarity in the medial temporal lobe: A three-component model. Trends in Cognitive Science, 11(9), 379-386. https://doi.org/10.1016/j.tics.2007.08.001

Dickerson, B. C., \& Eichenbaum, H. (2010). The episodic memory system: Neurocircuitry and disorders. Neuropsychopharmacology, 35(1), 86-104. https://doi.org/10.1038/npp.2009.126

Dobel, C., Lagemann, L., \& Zwitserlood, P. (2009). Non-native phonemes in adult word learning: Evidence from the N400m. Philosophical transactions of the Royal Society B: Biological Sciences, 364(1536), 3697-3709. https://doi.org/10.1098/rstb.2009.0158

Dudai, Y., \& Eisenberg, M. (2004). Rites of passage of the engram: Reconsolidation and the lingering consolidation hypothesis. Neuron, 44 (1), 93-100. https://doi.org/10.1016/j.neuron.2004.09.003

Duff, M. C., Covington, N. V., Hilverman, C., \& Cohen, N. J. (2020). Semantic memory and the hippocampus: Revisiting, reaffirming, and extending the reach of their critical relationship. Frontiers in Human Neuroscience, 13, 471. https://doi.org/10.3389/fnhum.2019.00471

Dumay, N., \& Gaskell, M. G. (2007). Sleep-associated changes in the mental representation of spoken words. Psychological Science, 18, 35-39. https://doi.org/10.1111/j.1467-9280.2007.01845.x

Dumay, N., \& Gaskell, M. G. (2012). Overnight lexical consolidation revealed by speech segmentation. Cognition, 123, 119-132. https://doi.org/10.1016/j.cognition.2011.12.009

Dumay, N., Gaskell, M. G., \& Feng, X. (2004). A day in the life of a spoken word. In K. Forbus, D. Gentner, \& T. Regier (Eds.), Proceedings of the Twenty-Sixth Annual Conference of the Cognitive Science Society (pp. 339-344). Erlbaum.

Eichenbaum, H., Yonelinas, A. P., \& Ranganath, C. (2007). The medial temporal lobe and recognition memory. Annual Review of Neuroscience, 30, 123-152. https://doi.org/10.1146/annurev.neuro.30.051606.094328

Elgort, I. (2011). Deliberate learning and vocabulary acquisition in a second language. Language Learning, 61, 367-413. https://doi.org/10.1111/j.14679922.2010.00613.x

Elgort, I., \& Warren, P. (2014). L2 vocabulary learning from reading: Explicit and tacit lexical knowledge and the role of learner and item variables. Language Learning, 64 (2), 365-414. https://doi.org/10.1111/lang.12052

Fang, X., \& Perfetti, C. (2017). Perturbation of old knowledge precedes integration of new knowledge. Neuropsychologia, 99, 270-278. https://doi.org/10.1016/j.neuropsychologia.2017.03.015

Fodor, J. A. (1983). The Modularity of Mind. MIT Press.

Friederici, A. D. (2002). Towards a neural basis of auditory sentence processing. Trends in Cognitive Sciences, 6(2), 78-84. https://doi.org/10.1016/s1364-6613(00)01839-8

Gaskell, M. G., \& Dumay, N. (2003). Lexical competition and the acquisition of novel words. Cognition, 89, 105-132. https://doi.org/10.1016/S0010-0277(03)00070-2 
Gelfand, A. E., \& Wang, F. (2002). A simulation-based approach to Bayesian sample size determination for performance under a given model and for separating models. Statistical Science, 17(2), 193-208. https://doi.org/10.1214/ss/1030550861

Geukes, S., Gaskell, M. G., \& Zwitserlood, P. (2015). Stroop effects from newly learned color words: Effects of memory consolidation and episodic context. Frontiers in Psychology, 6, 278. https://doi.org/10.3389/fpsyg.2015.00278

Grainger, J., \& Jacobs, A. M. (1996). Orthographic processing in visual word recognition: A multiple read-out model. Psychological Review, 103(3), 518-565. https://doi.org/10.1037/0033-295X.103.3.518

Gronau, Q. F., Singmann, H., \& Wagenmakers, E. (2020). bridgesampling: An R package for estimating normalizing constants. Journal of Statistical Software, 92(10), 1-29. https://doi.org/10.18637/jss.v092.i10

Groppe, D. M., Urbach, T. P., \& Kutas, M. (2011a). Mass univariate analysis of eventrelated brain potentials/fields I: A critical tutorial review. Psychophysiology, 48(12), 1711-1725. https://doi.org/10.1111/j.1469-8986.2011.01273.x

Groppe, D. M., Urbach, T. P., \& Kutas, M. (2011b). Mass univariate analysis of eventrelated brain potentials/fields II: Simulation studies. Psychophysiology, 48(12), 1726-1737. https://doi.org/10.1111/j.1469-8986.2011.01272.x

Hagoort, P. (2008). Should psychology ignore the language of the brain? Current Directions in Psychological Science, 17(2), 96-101. https://doi.org/10.1111/j.14678721.2008.00556.x

Hasselmo, M. E., Craik, F. I. M., \& Davachi, L. (2007). Encoding. In H. L. Roediger, Y. Dudai, \& S. M. Fitzpatrick (Eds.), Science of Memory: Concepts (pp. 122-144). Oxford University Press. https://doi.org/10.1093/acprof:oso/9780195310443.003.0007

Hauk, O., Coutout, C., Holden, A., \& Chen, Y. (2012). The time-course of singleword reading: Evidence from fast behavioral and brain responses. NeuroImage, 60, 1462-1477. https://doi.org/10.1016/j.neuroimage.2012.01.061

Heck, D., Böhm, U., Böing-Messing, F., Bürkner, P., Derks, K., Dienes, Z., Fu, Q., Gu, X., Karimova, D., Kiers, H. A. L., Klugkist, I., Kuiper, R. M., Lee, M. D., Leenders, R., Leplaa, H. J., Linde, M., Ly, A., Meijerink-Bosman, M., Moerbeek, M., ... Hoijtink, H. (2020). A review of applications of the Bayes factor in psychological research. https://doi.org/10.31234/osf.io/cu43g

Henderson, L. M., Weighall, A. R., Brown, H., \& Gaskell, M. G. (2012). Consolidation of vocabulary is associated with sleep in children. Developmental Science, 15(5), 674-687. https://doi.org/10.1111/j.1467-7687.2012.01172.x

Henderson, L. M., Weighall, A. R., Brown, H., \& Gaskell, M. G. (2013). Online lexical competition during spoken word recognition and word learning in children and adults. Child Development, 84(5), 1668-1685. https://doi.org/10.1111/cdev.12067

Hill, H., Strube, M., Roesch-Ely, D., \& Weisbrod, M. (2002). Automatic vs. controlled processes in semantic priming - differentiation by eventrelated potentials. International Journal of Psychophysiology, 44(3), 197-218. https://doi.org/10.1016/S0167-8760(01)00202-1

Holcomb, P. J., Grainger, J., \& O'Rourke, T. (2002). An electrophysiological study of the effects of orthographic neighborhood size on printed word perception. Journal of Cognitive Neuroscience, 14(6), 938-950. https://doi.org/10.1162/089892902760191153

Hoshino, N., \& Thierry, G. (2012). Do Spanish-English bilinguals have 
their fingers in two pies — or is it their toes? An electrophysiological investigation of semantic access in bilinguals. Frontiers in Psychology, 3, 1-6. https://doi.org/10.3389/fpsyg.2012.00009

Hutchison, K. A. (2003). Is semantic priming due to association strength or feature overlap? A microanalytic review. Psychonomic Bulletin \& Review, 10, 785-813. http://dx.doi.org/10.3758/BF03196544

Ince, E., \& Christman, S. D. (2002). Semantic representations of word meanings by the cerebral hemispheres. Brain and Language, 80(3), 393-420. https://doi.org/10.1006/brln.2001.2599

James, E., Gaskell, M. G., \& Henderson, L. M. (2019). Offline consolidation supersedes prior knowledge benefits in children's (but not adults') word learning. Developmental Science, 22, e12776. https://doi.org/10.1111/desc.12776

James, E., Gaskell, M. G., Weighall, A. R., \& Henderson, L. M. (2017). Consolidation of vocabulary during sleep: The rich get richer? Neuroscience \& Biobehavioral Reviews, 77, 1-13. https://doi.org/10.1016/j.neubiorev.2017.01.054

Jeffreys, H. (1939). Theory of Probability. Clarendon Press.

Kandhadai, P., \& Federmeier, K. D. (2010). Automatic and controlled aspects of lexical associative processing in the two cerebral hemispheres. Psychophysiology, $47(4)$, 774-785. https://doi.org/10.1111/j.1469-8986.2009.00969.x

Kapnoula, E., \& McMurray, B. (2016). Newly learned word forms are abstract and integrated immediately after acquisition. Psychonomic Bulletin \& Review, 23, 491499. https://doi.org/10.3758/s13423-015-0897-1

Kapnoula, E., Packard, S., Gupta, P., \& McMurray, B. (2015). Immediate lexical integration of novel word forms. Cognition, 134, 85-99. https://doi.org/10.1016/j.cognition.2014.09.007

Kass, R. E., \& Raftery, A. E. (1995). Bayes factors. Journal of the American Statistical Association, 90(430), 773-795. https://doi.org/10.2307/2291091

Kazmerski, V. A., \& Friedman, D. (1997). Old/new differences in direct and indirect memory tests using pictures and words in within- and cross-form conditions: Eventrelated potential and behavioral measures. Cognitive Brain Research, 5(4), 255-272. https://doi.org/10.1016/S0926-6410(97)00004-9

Keuleers, E., Stevens, M., Mandera, P., \& Brysbaert, M. (2015). Word knowledge in the crowd: Measuring vocabulary size and word prevalence in a massive online experiment. Quarterly Journal of Experimental Psychology, 68(8), 1665-1692. https://doi.org/10.1080/17470218.2015.1022560

Korochkina, M., Bürki, A., \& Nickels, L. (2021). Apples and oranges: How does learning context affect novel word learning? Journal of Memory and Language, 120, 104246. https://doi.org/10.1016/j.jml.2021.104246

Kumar, A. A. (2021). Semantic memory: A review of methods, models, and current challenges. Psychonomic Bulletin \& Review, 28, 40-80. https://doi.org/10.3758/s13423-020-01792-x

Kumaran, D., Hassabis, D., \& McClelland, J. L. (2016). What learning systems do intelligent agents need? Complementary learning systems theory updated. Trends in Cognitive Sciences, 20, 512-534. https://doi.org/10.1016/j.tics.2016.05.004

Kurdziel, L. B. F., Mantua, J., \& Spencer, R. M. C. (2017). Novel word learning in older adults: A role for sleep? Brain and Language, 167, 106-113. http://dx.doi.org/10.1016/j.bandl.2016.05.010

Kutas, M., \& Federmeier, K. D. (2000). Electrophysiology reveals semantic memory 
use in language comprehension. Trends in Cognitive Sciences, 4(12), 463-470. https://doi.org/10.1016/s1364-6613(00)01560-6

Kutas, M., \& Federmeier, K. D. (2011). Thirty years and counting: Finding meaning in the N400 component of the event-related brain potential (ERP). Annual Review of Psychology, 62, 621-647. https://doi.org/10.1146/annurev.psych.093008.131123

Lau, E. F., Phillips, C., \& Poeppel, D. (2008). A cortical network for semantics: (De)constructing the N400. Nature Review Neuroscience, 9(12), 920-933. https://doi.org/10.1038/nrn2532

Leach, L., \& Samuel, A. (2007). Lexical configuration and lexical engagement: When adults learn new words. Cognitive Psychology, 55, 306-353. https://doi.org/10.1016/j.cogpsych.2007.01.001

Lerner, I., Bentin, S., \& Shriki, O. (2012). Spreading activation in an attractor network with latching dynamics: Automatic semantic priming revisited. Cognitive Science, 36(8), 1339-1382. https://doi.org/10.1111/cogs.12007

Lewandowski, D., Kurowicka, D., \& Joe, H. (2009). Generating random correlation matrices based on vines and extended onion method. Journal of Multivariate Analysis, 100(9), 1989-2001. https://doi.org/10.1016/j.jmva.2009.04.008

Lilford, R. J., Thornton, J. G., \& Braunholtz, D. (1995). Clinical trials and rare diseases - a way out of a conundrum. British Medical Journal, 311(7020), 1621-1625. https://doi.org/10.1136/bmj.311.7020.1621

Lindsay, S., \& Gaskell, M. (2013). Lexical integration of novel words without sleep. Journal of Experimental Psychology: Learning, Memory, and Cognition, 39, 608622. https://doi.org/10.1037/a0029243

Lindsay, S., \& Gaskell, M. G. (2010). A Complementary Systems Account of word learning in L1 and L2. Language Learning, 60, 45-63. https://doi.org/10.1111/j.14679922.2010.00600.x

Liu, Y., \& van Hell, J. (2020). Learning novel word meanings: An ERP study on lexical consolidation in monolingual, inexperienced foreign language learners. Language Learning, 70 (S2), 45-74. https://doi.org/10.1111/lang.12403

Lucas, M. (2000). Semantic priming without association: A metaanalytic review. Psychonomic Bulletin \& Review, 7, 618-630. http://dx.doi.org/10.3758/BF03212999

McCandliss, B. D., Cohen, L., \& Dehaene, S. (2003). The visual word form area: Expertise for reading in the fusiform gyrus. Trends in Cognitive Sciences, 7(7), 293-299. https://doi.org/10.1016/s1364-6613(03)00134-7

McClelland, J. L. (2013). Incorporating rapid neocortical learning of new schema-consistent information into complementary learning systems theory. Journal of Experimental Psychology: General, 142, 1190-1210. https://doi.org/10.1037/a0033812

McClelland, J. L., McNaughton, B. L., \& Lampinen, A. K. (2020). Integration of new information in memory: New insights from a complementary learning systems perspective. Philosophical Transactions of the Royal Society B: Biological Sciences, 375, 20190637. http://dx.doi.org/10.1098/rstb.2019.0637

McClelland, J. L., McNaughton, B. L., \& O'Reilly, R. C. (1995). Why there are complementary learning systems in the hippocampus and neocortex: Insights from the successes and failures of connectionist models of learning and memory. Psychological Review, 102, 419-457. https://doi.org/10.1037/0033295X.102.3.419 
McGregor, K. K. (2014). What a difference a day makes: Change in memory for newly learned word forms over twenty-four hours. Journal of Speech, Language, and Hearing Research, 57, 1842-1850. https://doi.org/10.1044/2014_JSLHR-L-130273

McLaughlin, J., Osterhout, L., \& Kim, A. (2004). Neural correlates of second language learning: Minimal instruction produces rapid change. Nature Neuroscience, 7, 703704. https://doi.org/10.1038/nn1264

McMurray, B., Kapnoula, E., \& Gaskell, M. G. (2016). Learning and integration of new word-forms: Consolidation, pruning, and the emergence of automaticity. In M. G. Gaskell \& J. Mirković (Eds.), Speech Perception and Spoken Word Recognition (pp. 116-142). Psychology Press.

McNamara, T. P. (2005). Semantic Priming: Perspectives from Memory and Word Recognition. Psychology Press.

McNamara, T. P., \& Altarriba, J. (1988). Depth of spreading activation revisited: Semantic mediated priming occurs in lexical decisions. Journal of Memory and Language, 27(5), 545-559. https://doi.org/10.1016/0749-596X(88)90025-3

McRae, K., \& Boisvert, S. (1998). Automatic semantic similarity priming. Journal of Experimental Psychology: Learning, Memory, and Cognition, 24, 558-572. http://dx.doi.org/10.1037/0278-7393.24.3.558

McRae, K., de Sa, V., \& Seidenberg, M. S. (1997). On the nature and scope of featural representations of word meaning. Journal of Experimental Psychology: General, 126(2), 99-130. https://doi.org/10.1037//0096-3445.126.2.99

Medler, D. A., \& Binder, J. R. (2008). MCWord: An On-Line Orthographic Database of the English Language. http://www.neuro.mcw.edu/mcword/

Meeter, M., \& Murre, J. M. J. (2004). Consolidation of long-term memory: Evidence and alternatives. Psychological Bulletin, 130(6), 843-857. https://doi.org/10.1037/0033-2909.130.6.843

Meng, X., \& Wong, W. H. (1996). Simulating ratios of normalizing constants via a simple identity: A theoretical exploration. Statistica Sinica, 6(4), 831-860. http://www.jstor.org/stable/24306045

Mestres-Missé, A., Camara, E., Rodriguez-Fornells, A., Rotte, M., \& Munte, T. F. (2008). Functional neuroanatomy of meaning acquisition from context. Journal of Cognitive Neuroscience, 20, 2153-2166. https://doi.org/10.1162/jocn.2008.20150

Mestres-Missé, A., Rodriguez-Fornells, A., \& Munte, T. F. (2007). Watching the brain during meaning acquisition. Cerebral Cortex, 17, 1858-1866. https://doi.org/10.1093/cercor/bhl094

Morey, R. (2008). Confidence intervals from normalized data: A correction to Cousineau (2005). Tutorials in Quantitative Methods for Psychology, 4(2), 61-64. https://doi.org/10.20982/tqmp.01.1.p042

Moscovitch, M., Cabeza, R., Winocur, G., \& Nadel, L. (2016). Episodic memory and beyond: The hippocampus and neocortex in transformation. Annual Review of Psychology, 67, 105-134. https://doi.org/10.1146/annurev-psych-113011-143733

Moss, H. E., Ostrin, R. K., Tyler, L. K., \& Marslen-Wilson, W. D. (1995). Accessing different types of lexical semantic information: Evidence from priming. Journal of Experimental Psychology: Learning, Memory, and Cognition, 21(4), 863-883. https://doi.org/10.1037/0278-7393.21.4.863

Neely, J. H. (1991). Semantic priming effects in visual word recognition: A selective review of current findings and theories. In D. Besner \& G. W. Humphreys (Eds.), 
Basic Processes in Reading: Visual Word Recognition (pp. 264-336). Erlbaum.

Nicenboim, B., \& Vasishth, S. (2016). Statistical methods for linguistic research:

Foundational Ideas - Part II. Language and Linguistics Compass, 10, 591-613. https://doi.org/10.1111/lnc3.12207

Nieuwland, M. S., Barr, D. J., Bartolozzi, F., Busch-Moreno, S., Darley, E., Donaldson, D. I., Ferguson, H. J., Fu, X., Heyselaar, E., Huettig, F., Husband, E. M., Ito, A., Kazanina, N., Kogan, V., Kohút, Z., Kulakova, E., Mézière, D., Politzer-Ahles, S., Rousselet, G., ... Von Grebmer Zu Wolfsthurn, S. (2019). Lexical prediction in the aging brain: The effects of predictiveness and congruency on the N400 ERP component. Philosophical Transactions of the Royal Society B: Biological Sciences, 375: 20180522. https://dx.doi.org/10.1098/rstb.2018.0522

Olichney, J. M., Van Petten, C., Paller, K. A., Salmon, D. P., Iragui, V. J., \& Kutas, M. (2000). Word repetition in amnesia. Brain, 123(9), 1948-1963. https://doi.org/10.1093/brain/123.9.1948

O'Reilly, R. C., Bhattacharyya, R., Howard, M. D., \& Ketz, N. (2014). Complementary learning systems. Cognitive Science, 38(6), 1229-1248. https://doi.org/10.1111/j.1551-6709.2011.01214.x

Paller, K. A., Kutas, M., \& McIsaac, H. K. (1995). Monitoring conscious recollection via the electrical activity of the brain. Psychological Science, 6(2), 107-111. https://doi.org/10.1111/j.1467-9280.1995.tb00315.x

Paller, K. A., Voss, J. L., \& Boehm, S. G. (2007). Validating neural correlates of familiarity. Trends in Cognitive Science, 11(6), 243-250. https://doi.org/10.1016/j.tics.2007.04.002

Payne, B. R., Lee, C., \& Federmeier, K. D. (2015). Revisiting the incremental effects of context on word processing: Evidence from singleword event-related brain potentials. Psychophysiology, 52(11), 1456-1469. https://dx.doi.org/10.1111/psyp.12515

Perfetti, C. A., Wlotko, E. W., \& Hart, L. A. (2005). Word learning and individual differences in word learning reflected in event-related potentials. Journal of Experimental Psychology: Learning, Memory, and Cognition, 31(6), 1281-1292. https://doi.org/10.1037/0278-7393.31.6.1281

Pernet, C. R., Chauveau, N., Gaspar, C., \& Rousselet, G. A. (2011). LIMO EEG: A toolbox for hierarchical LInear MOdeling of ElectroEncephaloGraphic data. Computational Intelligence and Neuroscience, 11, e831409. https://doi.org/10.1155/2011/831409

Pernet, C. R., Latinus, M., Nichols, T. E., \& Rousselet, G. A. (2015). Clusterbased computational methods for mass univariate analyses of event-related brain potentials/fields: A simulation study. Journal of Neuroscience Methods, 250 (Supplement C), 85-93. https://doi.org/10.1016/j.jneumeth.2014.08.003

Peters, J., \& Daum, I. (2009). Frontal but not parietal positivity during source recollection is sensitive to episodic content. Neuroscience Letters, 454(3), 182-186. https://doi.org/10.1016/j.neulet.2009.03.019

Pion-Tonachini, L., Kreutz-Delgado, K., \& Makeig, S. (2019). ICLabel: An automated electroencephalographic independent component classifier, dataset, and website. NeuroImage, 198, 181-197. https://doi.org/10.1016/j.neuroimage.2019.05.026

Posner, M. I., \& Snyder, C. R. R. (1975). Attention and cognitive control. In R. L. Solso (Ed.), Information Processing and Cognition: The Loyola Symposium (pp. 55-85). Erlbaum. 
Pulvermüller, F., Shtyrov, Y., \& Hauk, O. (2009). Understanding in an instant: Neurophysiological evidence for mechanistic language circuits in the brain. Brain and Language, 110(2), 81-94. https://doi.org/10.1016/j.bandl.2008.12.001

Qiao, X., Forster, K., \& Witzel, N. (2009). Is banara really a word? Cognition, 113, 254-257. https://doi.org/10.1016/j.cognition.2009.08.006

R Core Team. (2021). R: A language and environment for statistical computing [Computer software manual]. Vienna, Austria. https://www.R-project.org/

Rabovsky, M., Hansen, S. S., \& McClelland, J. L. (2018). Modelling the N400 brain potential as change in a probabilistic representation of meaning. Nature Human Behaviour, 2, 693-705. https://doi.org/10.1038/s41562-018-0406-4

Rabovsky, M., \& McClelland, J. L. (2020). Quasi-compositional mapping from form to meaning: A neural network-based approach to capturing neural responses during human language comprehension. Philosophical Transactions of the Royal Society B: Biological Sciences, 375(1791), 20190313. https://doi.org/10.1098/rstb.2019.0313

Rasch, B., \& Born, J. (2013). About sleep's role in memory. Physiological Reviews, 93, 681e766. https://doi.org/10.1152/physrev.00032.2012

Ratcliff, R., \& McKoon, G. (1988). A retrieval theory of priming in memory. Psychological Review, 95(3), 385-408. https://doi.org/10.1037/0033-295X.95.3.385

Rigby, R. A., \& Stasinopoulos, D. M. (2005). Generalized additive models for location, scale and shape. Journal of the Royal Statistical Society: Series C (Applied Statistics), 54(3), 507-554. https://doi.org/10.1111/j.1467-9876.2005.00510.x

Rugg, M. D. (1983). Further study of the electrophysiological correlates of lexical decision. Brain and Language, 19(1), 142-152. https://doi.org/10.1016/0093-934x(83)900603

Rugg, M. D. (1995). ERP studies of memory. In M. D. Rugg \& M. G. H. Coles (Eds.), Electrophysiology of Mind: Event-Related Brain Potentials and Cognition (pp. 133170). Oxford University Press.

Rugg, M. D., \& Curran, T. (2007). Event-related potentials and recognition memory. Trends in Cognitive Sciences, 11(6), 251-257. https://doi.org/10.1016/j.tics.2007.04.004

Schad, D., Nicenboim, B., Betancourt, M., \& Vasishth, S. (2021). Toward a principled Bayesian workflow in cognitive science. Psychological Methods, 26(1), 103-126. https://doi.org/10.1037/met0000275

Schad, D., Nicenboim, B., Bürkner, P., Betancourt, M., \& Vasishth, S. (2021). Workflow techniques for robust use of Bayes factors. arXiv:2103.08744v2

Schönbrodt, F. D., \& Wagenmakers, E. (2018). Bayes factor design analysis: Planning for compelling evidence. Psychonomic Bulletin \& Review, 25(1), 128142. https://doi.org/10.3758/s13423-017-1230-y

Senkfor, A. J., \& Van Petten, C. (1998). Who said what? An eventrelated potential investigation of source and item memory. Journal of Experimental Psychology: Learning, Memory, and Cognition, 24(4), 1005-1025. https://doi.org/10.1037/0278-7393.24.4.1005

Shelton, J. R., \& Martin, R. C. (1992). How semantic is automatic semantic priming? Journal of Experimental Psychology: Learning, Memory, and Cognition, 18(6), 1191-1210. https://doi.org/10.1037/0278-7393.18.6.1191

Smith, S., \& Nichols, T. (2009). Threshold-free cluster enhancement: Addressing problems of smoothing, threshold dependence and localisation in cluster inference. NeuroImage, 44(1), 83-98. https://doi.org/10.1016/j.neuroimage.2008.03.061 
Sparks, J. R., \& Rapp, D. N. (2010). Discourse processing - examining our everyday language experiences. Wiley Interdisciplinary Reviews Cognitive Science, 1, 371381. https://doi.org/10.1002/wcs.11

Spiegelhalter, D. J., Abrams, K. R., \& Myles, J. R. (2003). Bayesian Approaches to Clinical Trials and Health-Care Evaluation. John Wiley \& Sons, Ltd. https://doi.org/10.1002/0470092602

Squire, L. R. (2004). Memory systems of the brain: A brief history and current perspective. Neurobiology of Learning and Memory, 82(3), 1710177. https://doi.org/10.1016/j.nlm.2004.06.005

Stan Development Team. (2017). Stan Modeling Language Users Guide and Reference Manual, Version 2.21.2 [Computer software manual]. http://mc-stan.org/

Stickgold, R., \& Walker, M. P. (2005). Memory consolidation and reconsolidation: What is the role of sleep? Trends in Neurosciences, 28(8), 408-415. https://doi.org/10.1016/j.tins.2005.06.004

Swaab, T. Y., Brown, C., \& Hagoort, P. (1998). Understanding ambiguous words in sentence contexts: Electrophysiological evidence for delayed contextual selection in Broca's aphasia. Neuropsychologia, 36(8), 737-761. https://doi.org/10.1016/S0028-3932(97)00174-7

Szewczyk, J. M., \& Schriefers, H. (2018). The N400 as an index of lexical preactivation and its implications for prediction in language comprehension. Language, Cognition and Neuroscience, 33(6), 665-686. https://doi.org/10.1080/23273798.2017.1401101

Tamminen, J., \& Gaskell, M. G. (2008). Newly learned spoken words show long-term lexical competition effects. The Quarterly Journal of Experimental Psychology, 61(3), 361-371. https://doi.org/10.1080/17470210701634545

Tamminen, J., \& Gaskell, M. G. (2013). Novel word integration in the mental lexicon: Evidence from unmasked and masked semantic priming. The Quarterly Journal of Experimental Psychology, 66(5), 1001-1025. https://doi.org/10.1080/17470218.2012.724694

Tamminen, J., Payne, J., Stickgold, R., Wamsley, E., \& Gaskell, M. G. (2010). Sleep spindle activity is associated with the integration of new memories and existing knowledge. Journal of Neuroscience, 30, 14356-14360. https://doi.org/10.1523/JNEUROSCI.3028-10.2010

Tham, E., Lindsay, S., \& Gaskell, M. G. (2015). Markers of automaticity in sleep-associated consolidation of novel words. Neuropsychologia, 71, 146-157. https://doi.org/10.1016/j.neuropsychologia.2015.03.025

Tompkins, C. A., Scharp, V. L., Meigh, K. M., \& Fassbinder, W. (2008). Coarse coding and discourse comprehension in adults with right hemisphere brain damage. Aphasiology, 22(2), 204-223. https://doi.org/10.1080/02687030601125019

van Berkum, J. J. (2009). The 'neuropragmatics' of simple utterance comprehension: An ERP review. In U. Sauerland \& K. Yatsushiro (Eds.), Semantics and Pragmatics: From Experiment to Theory (pp. 276-316). Palgrave Macmillan.

van Berkum, J. J., Hagoort, P., \& Brown, C. M. (1999). Semantic integration in sentences and discourse: Evidence from the N400. Journal of Cognitive Neuroscience, 11 (6), 657-671. https://doi.org/10.1162/089892999563724

van der Ven, F., Takashima, A., Segers, E., \& Verhoeven, L. (2015). Learning word meanings: Overnight integration and study modality effects. PLoS ONE, 10, e0124926. https://doi.org/10.1371/journal.pone.0124926

Van Petten, C., Kutas, M., Kluender, R., Mitchiner, M., \& McIsaac, H. (1991). 
Fractionating the word repetition effect with event-related potentials. Journal of Cognitive Neuroscience, 3(2), 131-150. https://doi.org/10.1162/jocn.1991.3.2.131

Van Petten, C., \& Luka, B. J. (2012). Prediction during language comprehension: Benefits, costs, and ERP components. International Journal of Psychophysiology, 83(2), 176-190. https://doi.org/10.1016/j.ijpsycho.2011.09.015

Van Petten, C., \& Senkfor, A. J. (1996). Memory for words and novel visual patterns: Repetition, recognition, and encoding effects in the event-related brain potential. Psychophysiology, 33(5), 491-506. https://doi.org/10.1111/j.14698986.1996.tb02425.x

Voss, J. L., \& Paller, K. A. (2007). Neural correlates of conceptual implicit memory and their contamination of putative neural correlates of explicit memory. Learning $\&$ Memory, 14(4), 259-267. https://doi.org/10.1101/lm.529807

Walker, S., Henderson, L. M., Fletcher, F., Knowland, V., Cairney, S. A., \& Gaskell, M. G. (2010). Learning to live with interfering neighbours: The influence of time of learning and level of encoding on word learning. Royal Society Open Science, 6, 181842. https://doi.org/10.1098/rsos.181842

Weighall, A. R., Henderson, L. M., Barr, D. J., Cairney, S. A., \& Gaskell, M. G. (2016). Eye-tracking the time-course of novel word learning and lexical competition in adults and children. Brain and Language, 167, 13-27. https://doi.org/10.1016/j.bandl.2016.07.010

Wilcox, R. R. (2005). Introduction to Robust Estimation and Hypothesis Testing. Academic Press.

Winocur, G., \& Moscovitch, M. (2011). Memory transformation and systems consolidation. Journal of the International Neuropsychological Society, 17, 766780. https://doi.org/10.1017/S1355617711000683

Wixted, J. T. (2004). The psychology and neuroscience of forgetting. Annual Review of Psychology, 55, 235-269. https://doi.org/10.1146/annurev.psych.55.090902.141555

Yarkoni, T., Balota, D., \& Yap, M. (2008). Moving beyond Coltheart's N: A new measure of orthographic similarity. Psychonomic Bulletin $\&$ Review, 15, 971-979. https://doi.org/10.3758/PBR.15.5.971

Yonelinas, A. P. (2002). The nature of recollection and familiarity: A review of 30 years of research. Journal of Memory and Language, 46(3), 441-517. https://doi.org/10.1006/jmla.2002.2864

Yu, S. S., \& Rugg, M. D. (2010). Dissociation of the electrophysiological correlates of familiarity strength and item repetition. Brain Research, 1320, 74-84. https://doi.org/10.1016/j.brainres.2009.12.071 